\title{
Modellgestützte Wirkungsanalysen ausgewählter Maßnahmen und Strategien
}

\author{
Sarah Baum, Tobias Conradt, René Dechow, Peter Elsasser, Hermann \\ Englert, Nils Ermisch, Horst Gömann, Roland Goetzke, Pia Gottschalk, \\ Martin Gutsch, Martin Henseler, Jana Hoymann, Margret Köthke, \\ Peter Kreins, Petra Lasch-Born, Felicitas Suckow und Frank Wechsung
}

\section{Inhaltsverzeichnis}

4.1 Konzept der Wirkungsanalysen . . . . . . . . . . . . . . . . . . . . . . . . 145

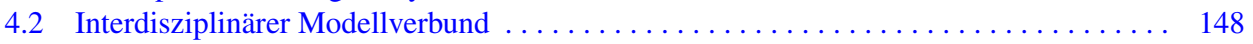

4.2.1 Aufbau des Modellverbunds . . . . . . . . . . . . . . . . . . . . . . . . . . 148

4.2.2 Modellentwicklungen und Simulationskonzepte. . . . . . . . . . . . . 150

4.2.2.1 Landnutzungsmodellierung mit dem Land Use Scanner . . . . . . . . 150

4.2.2.2 Modellierung der landwirtschaftlichen Landnutzung mit

RAUMIS .......................... 153

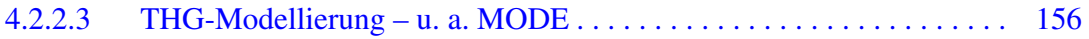

4.2.2.4 Entwicklung des Forstbetrieblichen Simulationsmodells FoBeSiMo. . . . . . . . . . . . . . . . . . . . . . . . . . . 159

4.2.2.5 Modellierung der Stoffflüsse im Forst mit 4C . . . . . . . . . . . . . . 164

4.3 Einordnung der verwendeten Klimaszenarien . . . . . . . . . . . . . . . . . . . 165

4.4 Ergebnisse der Siedlungs- und Verkehrsflächenmodellierung . . . . . . . . . . . . . 172

4.4.1 Referenzprojektion Flächennutzung . . . . . . . . . . . . . . . . . . 173

4.4.2 Analyse ausgewählter Maßnahmen ,Siedlung und Verkehr“ . . . . . . . . . . 174

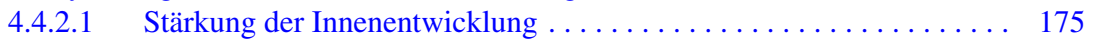

4.4.2.2 Ausschöpfung baulicher Dichte im Neubau . . . . . . . . . . . . . 182

4.4.2.3 Reduzierung der Flächeninanspruchnahme durch Verkehr . . . . . . 184

H. Gömann $(\bowtie) \cdot$ S. Baum · M. Henseler · P. Kreins

Thünen-Institut für Ländliche Räume, Braunschweig, Deutschland

E-Mail: cc-landstrad@thuenen.de

T. Conradt · P. Gottschalk · M. Gutsch · P. Lasch-Born · F. Suckow · F. Wechsung

Potsdam-Institut für Klimafolgenforschung e. V., Potsdam, Deutschland 


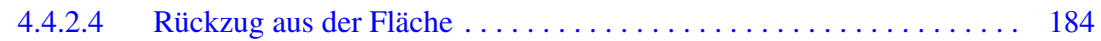

4.4.2.5 Erhalt und Entwicklung innerstädtischer Freiflächen. . . . . . . . . . . 187

4.4.2.6 Stärkung des ÖPNV . . . . . . . . . . . . . . . . . . . . . . . . . 191

4.4.2.7 Zusätzliche Ausweisung von Vorrang- und Vorbehaltsgebieten. . . . . . 195

4.4.2.8 Stärkung des Hochwasserschutzes . . . . . . . . . . . . . . . . . 197

4.4.2.9 Restriktiver Freiraumschutz . . . . . . . . . . . . . . . . . . . . 198

4.4.2.10 Energieerzeugung auf für Siedlungszwecke ungeeigneten

nicht-agrarischen Flächen. . . . . . . . . . . . . . . 200

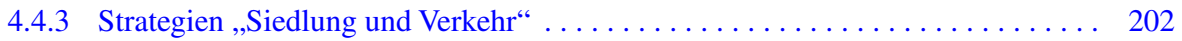

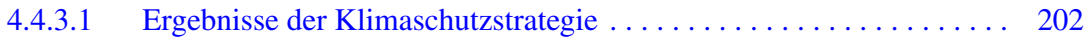

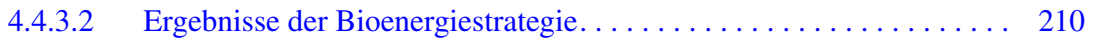

4.4.3.3 Ergebnisse der Natur- und Umweltschutzstrategie. . . . . . . . . . . . 210

4.4.3.4 Ergebnisse der Klimaanpassungsstrategie . . . . . . . . . . . . 213

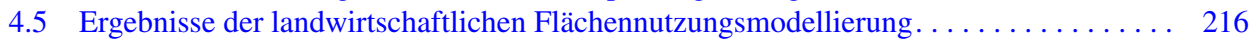

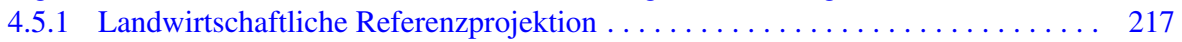

4.5.2 Analyse ausgewählter landwirtschaftlicher Maßnahmen. . . . . . . . . . . . 220

4.5.2.1 Anpassung der Nutzung organischer Böden. . . . . . . . . . . . . . 220

4.5.2.2 Anpassung des Düngemanagements............... 222

4.5.2.3 Substitution fossiler Energie durch Bioenergie . . . . . . . . . 225

4.5.2.4 Erhalt und Schaffung von Treibhausgassenken ... . . . . . . . . . 237

4.5.2.5 Vergleichende Beurteilung der Maßnahmen. . . . . . . . . . . . 242

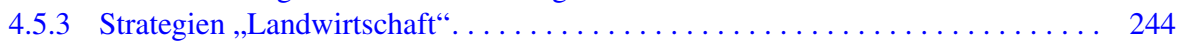

4.5.3.1 Ergebnisse der Klimaschutzstrategie . . . . . . . . . . . . 245

4.5.3.2 Ergebnisse der Bioenergiestrategie. . . . . . . . . . . . . . . . 249

4.5.3.3 Ergebnisse der Natur- und Umweltschutzstrategie. . . . . . . . . . 255

4.5.4 Vergleichende Beurteilung der landwirtschaftlichen Strategien . . . . . . . . . 258

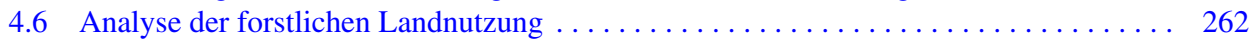

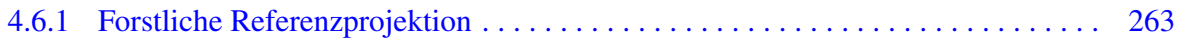

4.6.2 Strategien im Bereich „Forstwirtschaft“ . . . . . . . . . . . . . . . . . 267

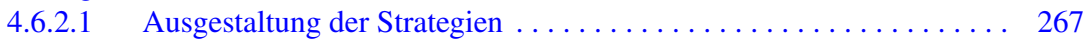

4.6.2.2 Ergebnisse der forstbetrieblichen Modellierung für die Strategien . . . 269

4.6.2.3 Exkurs: Klimaschutz durch Ausdehnung der Waldfläche und durch

Nutzungsverzichte im Wald $\ldots \ldots \ldots \ldots \ldots \ldots \ldots \ldots \ldots \ldots \ldots \ldots$

R. Dechow

Thünen-Institut für Agrarklimaschutz, Braunschweig, Deutschland

P. Elsasser · H. Englert · N. Ermisch · M. Köthke

Thünen-Institut für Internationale Waldwirtschaft und Forstökonomie, Hamburg-Bergedorf, Deutschland

R. Goetzke · J. Hoymann

Bundesinstitut für Bau-, Stadt- und Raumforschung im Bundesamt für Bauwesen und

Raumordnung, Bonn, Deutschland 
4.7 Ergebnisse der Modellierung von Stoffflüssen im Forst . . . . . . . . . . . . . . . 279

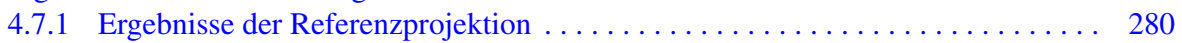

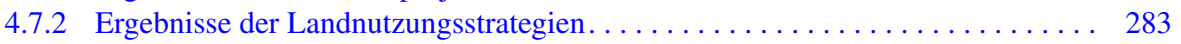

4.7.2.1 Klimaschutzstrategie...................... 283

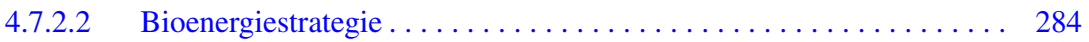

4.7.2.3 Natur- und Umweltschutzstrategie . . . . . . . . . . . . . . . 285

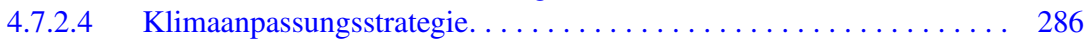

4.7.3 Vergleichende Beurteilung der Auswirkungen der Strategien . . . . . . . . . . . 288

\subsection{Konzept der Wirkungsanalysen}

Horst Gömann

\section{Zusammenfassung}

Im Dialog mit Akteuren der Landnutzung wurden die Leitmotive Klimaschutz, Bioenergieerzeugung, Umwelt- und Naturschutz und Klimaanpassung herausgearbeit. Diese werden in Landnutzungsstrategien untersucht und die möglichen Beiträge dieser Landnutzungsstrategien zu gesellschaftlichen Zielen geprüft. In der Strategie „Klimaschutz“ wird ermittelt, welchen Beitrag die Landnutzung zur Erreichung des Zieles leisten kann. Die Strategie „Bioenergie“ bündelt unterschiedliche Maßnahmen der Landnutzung, die die Produktion von Biomasse zur energetischen Verwendung steigern, um einen möglichst großen Beitrag zur Reduktion von THG-Emissionen sowie zur Erreichung des ErneuerbareEnergien-Ziels zu leisten. Die Strategie „Natur- und Umweltschutz“ fasst Maßnahmen der Landnutzung zur Reduzierung von THG-Emissionen bzw. zur Bindung von $\mathrm{CO}_{2}$ zusammen und die Strategie „Klimaanpassung“ orientiert sich an der 2008 durch die Bundesregierung verabschiedeten Deutschen Anpassungsstrategie (DAS) an den Klimawandel. Die Wirkungen der ausgewählten Maßnahmen und Landnutzungsstrategien wurden mit Hilfe des Modellverbundes im Rahmen eines komparativ-statischen Vergleichs untersucht.

In Kap. 3 wurden zahlreiche Maßnahmen der Landnutzung beschrieben, die einen Beitrag zum Klimaschutz leisten können. Es wurde darauf eingegangen, inwiefern sie im Rahmen des interdisziplinären Modellverbundes abgebildet sind oder sich durch eine Weiterentwicklung der Modelle abbilden lassen. Bei letzterem war stets der Aufwand für die Modellweiterentwicklung im Verhältnis zum erwarteten Minderungspotenzial der THGEmissionen abzuwägen. In der Regel adressieren sie nicht nur den Klimaschutz, sondern darüber hinaus weitere Ökosystemdienstleistungen. Aus diesem Abwägungsprozess resultiert eine Auswahl von Maßnahmen, die einen mehr oder minder ausgeprägten Beitrag zum Klimaschutz leisten. Je nach Schwerpunkt der Maßnahmen lassen sie sich unterschiedlichen gesellschaftlichen Ansprüchen an Landnutzung zuordnen. 
Tab. 4.1 Übersicht zur Zielausrichtung der untersuchten Strategien in CC-LandStraD. (Quelle: Fick et al. 2016)

\begin{tabular}{l|l}
\hline Strategie & Ziel(e) \\
\hline Klimaschutz & $\begin{array}{l}\text { Effiziente Emissionsreduktion/-bindung von } \mathrm{CO}_{2} \text {-Äquivalenten durch Land- } \\
\text { nutzung }\end{array}$ \\
\hline Bioenergie & $\begin{array}{l}\text { Erreichung der Bioenergieziele der Bundesregierung mit Abschätzung der } \\
\text { Emissionsreduktion/-bindung von } \mathrm{CO}_{2} \text {-Äquivalenten }\end{array}$ \\
\hline Natur- und & $\begin{array}{l}\text { Umsetzung der WRRL, EU-Flächenstilllegung, Biodiversitätsstrategie mit } \\
\text { Abschätzung der Emissionsreduktion/-bindung von } \mathrm{CO}_{2} \text {-Äquivalenten }\end{array}$ \\
\hline Klimaanpassung & $\begin{array}{l}\text { Reduzierung von klimawandelbedingten Risiken für die Landnutzung; } \\
\text { Zusätzliche Anstrengungen, die über die normale, kontinuierliche } \\
\text { Anpassung (z. B. technischer Fortschritt, Sortenwahl) hinausgehen }\end{array}$ \\
\hline
\end{tabular}

Im Rahmen des transdisziplinären Prozesses wurden folgende Leitmotive der Landnutzung herausgearbeit: Klimaschutz, Bioenergieerzeugung, Umwelt- und Naturschutz und Klimaanpassung. Diesen Leitmotiven wurden verschiedene Maßnahmen zu Maßnahmenbündeln zugeordnet, die nachfolgend als Landnutzungsstrategien bezeichnet werden. Es werden die möglichen Beiträge dieser Landnutzungsstrategien $\mathrm{zu}$ gesellschaftlichen Zielen untersucht, die von der Summe der jeweils einzelnen Maßnahmenbeiträge abweichen. Einen Überblick über die Landnutzungsstrategien und deren Ziele ist in Tab. 4.1 zusammengestellt. Die einzelnen Landnutzungsstrategien haben in den betrachteten landnutzenden Sektoren eine unterschiedliche Bedeutung.

In der Strategie ,Klimaschutz“" wird untersucht, welchen Beitrag die Landnutzung zur Erreichung des Zieles leisten kann. Es werden Maßnahmen gebündelt, die bei möglichst geringen Kosten möglichst viele $\mathrm{CO}_{2}$-Äquivalente reduzieren bzw. binden.

Die Strategie „Bioenergie“ bündelt unterschiedliche Maßnahmen der Landnutzung, die die Produktion von Biomasse zur energetischen Verwendung steigern, um einen möglichst großen Beitrag zur Reduktion von THG-Emissionen sowie zur Erreichung des Erneuerbare-Energien-Ziels zu leisten. Hierunter fällt beispielsweise der Einsatz alternativer Energiepflanzen in Form von Kurzumtriebsplantagen oder der Durchwachsenen Silphie. Derzeit bestehen folgende Verpflichtungen (BMELV und BMU 2010):

- Anteil der Biomasse zur Stromerzeugung aus erneuerbaren Energien bis 2030: etwa $12 \%^{1}$,

- Anteil der Biomasse zur Wärmeerzeugung aus erneuerbaren Energien bis 2020: 9,7 \%,

- Biokraftstoffanteil am Kraftstoffmarkt bis 2020: $7 \%$ der gesamten durch den Verkehr verursachten Emissionen einsparen.

\footnotetext{
${ }^{1}$ Ergibt sich aus der Vorgabe, dass 2020 die Stromerzeugung aus erneuerbaren Energien in Deutschland $35 \%$ beträgt, mit einem Anteil von $8 \%$ aus Biomasse. Darüber hinaus gibt es das Ziel, bis 2030 die Stromerzeugung aus erneuerbaren Energien in Deutschland auf $50 \% \mathrm{zu}$ erhöhen. Daraus ergibt sich im Verhältnis der Anteil zur Stromerzeugung aus Biomasse von $12 \%$.
} 
Im Siedlungsbereich wird die Bioenergiestrategie nicht mit eigenen flächenrelevanten Maßnahmen ausgestaltet. Allerdings können einzelne Bestandteile der in Abschn. 3.1 vorgestellten und in Abschn. 4.4 analysierten Maßnahmen einen Beitrag zur Bioenergiestrategie leisten, beispielsweise dadurch, dass sie durch die Verringerung des Siedlungsdrucks auf die Landwirtschaftsfläche die dortigen Nutzungskonflikte reduzieren.

Die Strategie ,Natur- und Umweltschutz“ bündelt Maßnahmen der Landnutzung zur Reduzierung von THG-Emissionen bzw. zur Bindung von $\mathrm{CO}_{2}$. Besondere Beachtung finden Belange des Natur- und Umweltschutzes, beispielsweise mittels einer möglichst hohen $\mathrm{CO}_{2}$-Bindung im Wald unter Berücksichtigung der Potenziellen Natürlichen Vegetation (PNV), oder der Reduzierung von $\mathrm{CO}_{2}$-Äquivalenten durch die angepasste Nutzung organischer Böden.

Die Strategie ,Klimaanpassung“ orientiert sich an der 2008 durch die Bundesregierung verabschiedeten Deutschen Anpassungsstrategie (DAS) an den Klimawandel. Damit soll mittels bundesweitem Handlungsrahmen Risiken aufgrund des Klimawandels vorgebeugt werden. Es werden Risiken für die Bevölkerung, für natürliche Lebensräume und die Volkswirtschaft benannt. Spezifiziert wird die Deutsche Anpassungsstrategie im Aktionsplan Anpassung (BMU 2012). Im Agrarsektor findet die Klimaanpassungsstrategie nur eingeschränkt Anwendung. Hier wird davon ausgegangen, dass aufgrund der überwiegend jährlichen Bewirtschaftungszyklen kontinuierlich Anpassungsmaßnahmen ergriffen werden, wie z. B. ein Wechsel der Anbauverfahren oder die Sortenwahl. Ein solches Vorgehen ist für die Forstwirtschaft als auch für das Siedlungswesen und die Verkehrsinfrastruktur aufgrund der langfristigen Produktions- bzw. Planungs-/Investitionshorizonte nicht möglich. Hier gilt es, vorausschauende Entscheidungen zur Klimaanpassung zu ergreifen, wie z. B. Überschwemmungsgebiete für den Hochwasserschutz auszuweisen oder Frischluftschneisen in Städten anzulegen oder zu erhalten. Von derartigen Maßnahmen wäre wiederum auch die Landwirtschaft betroffen, da für die Umsetzung der Maßnahmen in der Regel landwirtschaftlich genutzte Flächen benötigt werden.

Die Wirkungen der ausgewählten Maßnahmen und Landnutzungsstrategien wurden mit Hilfe des Modellverbundes im Rahmen eines komparativ-statischen Vergleichs untersucht. Dazu wurde in einem ersten Schritt eine Referenzprojektion bis zum Zieljahr 2030 modellgestützt projiziert. Diese basiert auf der Fortschreibung beobachteter Entwicklungen sowie zukünftig absehbarer bzw. zu erwartender Rahmenbedingungen. Die Referenzprojektion wurde über die betrachteten landnutzenden Sektoren abgestimmt und beinhaltet Ergebnisse von Projektionen globaler Entwicklungen aus dem Verbundvorhaben GLUES u. a. mit den Modellen DART und CAPRI (Delzeit et al. 2016). Die Ergebnisse der Referenzprojektion werden jeweils in den folgenden Abschnitten zu den einzelnen landnutzenden Sektoren Siedlung und Verkehr sowie Landwirtschaft und Wald dargestellt, damit ein unmittelbarer Bezug zu den Änderungen besteht, die die Landnutzungsmaßnahmen und -strategien bewirken.

Neben den vier Landnutzungsstrategien wird die Referenzprojektion bei den forstlichen Analysen mit zwei Klimaszenarien kombiniert und die Ergebnisse untereinander verglichen: dem Klima-Referenzprojektion (0K), welches keine Temperaturerhöhung ab 2007 vorschreibt, und dem Klima-Standardszenario RCP 8.5-ECHAM6, welches eine Temperaturerhöhung um $4,23^{\circ} \mathrm{K}$ im Mittel für Deutschland projiziert (vgl. Abb. 4.5). 


\subsection{Interdisziplinärer Modellverbund}

Horst Gömann, René Dechow, Nils Ermisch, Roland Goetzke, Martin Henseler, Jana Hoymann und Peter Kreins

\section{Zusammenfassung}

Mit Hilfe eines interdisziplinären Modellverbundes werden die Wirkungen der ausgewählten Maßnahmen und Landnutzungsstrategien in einem komparativ-statischen Vergleich betrachtet. Das Modell STAR (Statistical Regional Climate Model) regionalisiert Simulationsergebnisse globaler Klimamodelle auf der Basis von Trendinformationen und vorliegenden Beobachtungsdaten von Klimastationen. Die Klimadaten werden von den bio-physikalischen Prozessmodellen für THG-Emissionen (MODE) und den prozessbasierten Waldwachstumsmodellen (4C und SILVA) als Eingangsgrößen verwendet. Das Modell Land Use Scanner (LUS) nutzt regionale Raumansprüche und simuliert die Veränderung von Landnutzung und Landbedeckung. Die verfügbare landwirtschaftliche Fläche und Forstfläche bilden Eingangsgrößen für die Agrar- und Forstmodelle RAUMIS, FoBeSiMo sowie 4C. Das Regionalisierte Agrar- und UmweltInformationsSystem (RAUMIS) simuliert die regionalen Anpassungen der landwirtschaftlichen Landnutzung und Produktion sowie Auswirkungen auf Einkommen und Umweltindikatoren auf regionaler Ebene. Das Forstbetriebliches Simulationsmodell (FoBeSiMo) bildet forstliche Modellbetriebe mit ihren regionalspezifischen forstlichen Produktionsbedingungen und verschiedener Bewirtschaftungsstrategien ab.

Ein verbindendes Element der landnutzenden Sektoren Siedlung und Verkehr sowie Landwirtschaft und Wald ist der begrenzte Faktor „Fläche“. Darüber hinaus bestehen mit Blick auf die Wechselwirkungen zwischen Klima und Landnutzung, den jeweiligen Planungshorizonten von Landnutzungsmaßnahmen sowie der modellhaften Abbildung dieser Sektoren beträchtliche Unterschiede, die bei den Wirkungsanalysen zu berücksichtigen sind. Im folgenden Abschnitt wird ein Überblick über den Verbund interdisziplinärer Modelle gegeben.

\subsubsection{Aufbau des Modellverbunds}

\section{Horst Gömann}

Der Aufbau des Verbundes interdisziplinärer Modelle ist schematisch in Abb. 4.1 dargestellt. Das Modell STAR (Statistical Regional Climate Model; Orlowsky et al. 2008) regionalisiert Simulationsergebnisse globaler Klimamodelle auf der Basis von Trendinformationen und vorliegenden Beobachtungsdaten von Klimastationen. Die Klimadaten werden von den biophysikalischen Prozessmodellen für THG-Emissionen (MODE) und den prozessbasierten Waldwachstumsmodellen (4C und SILVA) als Eingangsgrößen genutzt.

Das Modell Land Use Scanner (LUS) (Hilferink und Rietveld 1999) nutzt regionale Raumansprüche, die aus verschiedenen sektoralen Langfristprognosen entnommen 


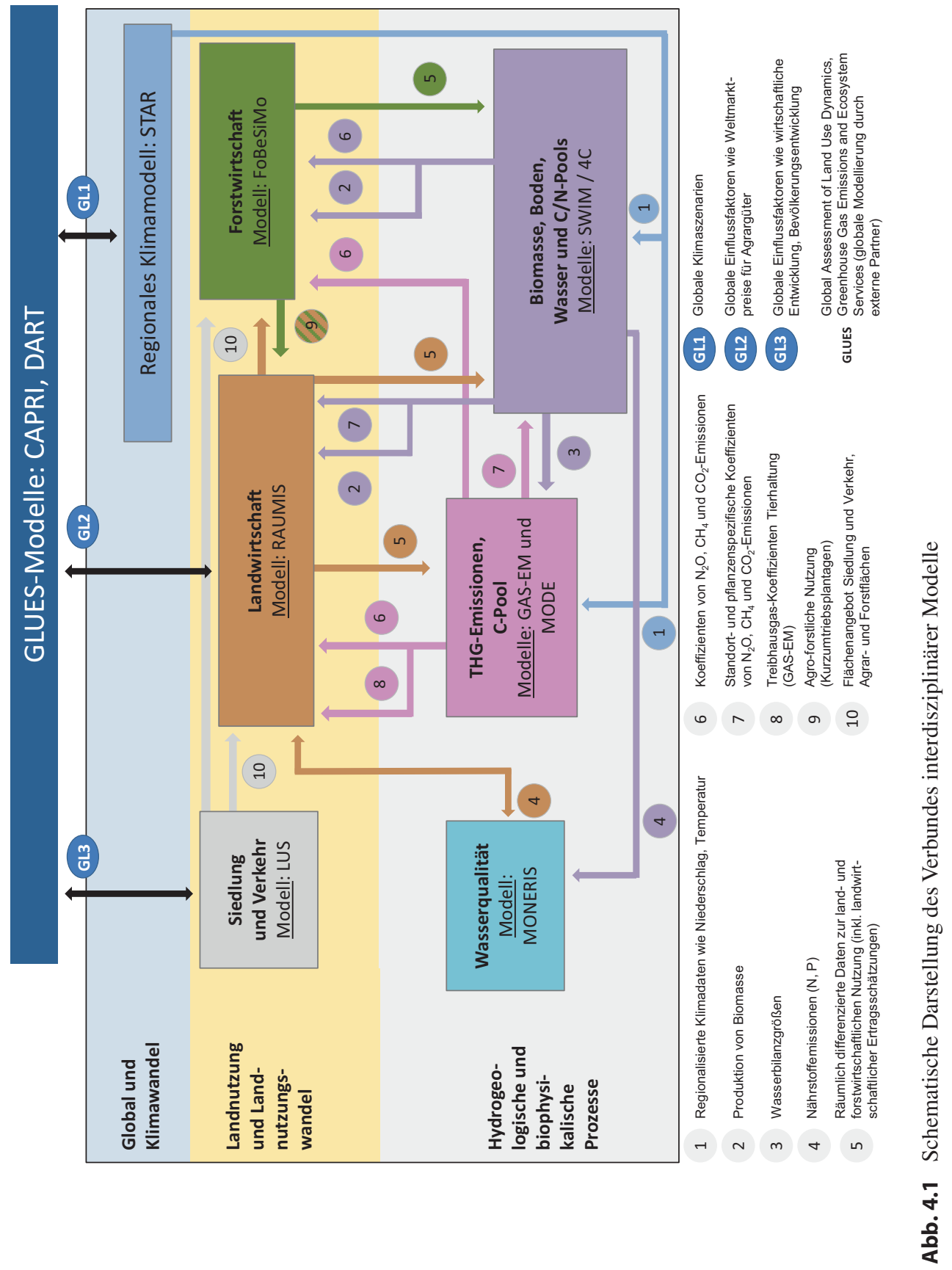


werden und simuliert die Veränderung von Landnutzung und Landbedeckung. Die verfügbare landwirtschaftliche Fläche und Forstfläche bilden Eingangsgrößen für die Agrarund Forstmodelle RAUMIS, FoBeSiMo sowie 4C.

Das Regionalisierte Agrar- und UmweltInformationsSystem (RAUMIS) (Henrichsmeyer et al. 1996) simuliert die regionalen Anpassungen der landwirtschaftlichen Landnutzung und Produktion sowie Auswirkungen auf Einkommen und Umweltindikatoren auf regionaler Ebene (Landkreise). Als Umweltindikatoren werden beispielsweise Nährstoffbilanzüberschüsse und THG-Emissionen ermittelt. Die Ergebnisse von RAUMIS werden ebenfalls zur Abschätzung der Auswirkungen auf die Biodiversität und bei der sozioökonomischen Evaluierung verwendet.

Das Forstbetriebliches Simulationsmodell (FoBeSiMo) simuliert forstliche Modellbetriebe mit ihren regionalspezifischen forstlichen Produktionsbedingungen und simuliert auf dieser Basis verschiedene forstliche Bewirtschaftungsstrategien. Unter Verwendung von empirischen Kennzahlen und Wirkungszusammenhängen werden Aussagen zum Marktverhalten deutscher Forstbetriebe unter sich ändernden Produktionsbedingungen gemacht. Die dafür notwendigen Klima-Eingangsdaten werden vom Modell C4 zur Verfügung gestellt. Über Informationen der agro-forstlichen Produktionsaktivitäten steht es im Austausch mit dem landwirtschaftlichen Modell RAUMIS.

Das Modell 4C (FORESt Ecosystems in a changing Environment) ist ein physiologisch basiertes Waldwachstumsmodell, das die Etablierung, das Wachstum und die Mortalität von Baumkohorten beschreibt (Lasch et al. 2005). Ergebnisparameter werden mit dem Modell FoBeSiMo ausgetauscht.

Das Modell MODE (Model Ensemble of fuzzy inference schemes) berechnet direkte $\mathrm{N}_{2} \mathrm{O}$ - und $\mathrm{CH}_{4}$ - Emissionen aus Böden, in Abhängigkeit von saisonalen Witterungsbedingungen, Bodeneigenschaften und Bewirtschaftungseinflüssen. Es dient der THG-Bilanzierung (Regionalisierung) und der Untersuchung natürlicher und anthropogener Einflüsse auf bodenbürtige $\mathrm{N}_{2} \mathrm{O}$ - und $\mathrm{CH}_{4}$-Emissionen. Weiterhin liefert MODE Emissionsinformationen an RAUMIS und FoBeSiMo.

GAS-EM ist ein modulares Tabellenkalkulationsprogramm zur Berechnung gasförmiger und partikelförmiger Emissionen aus Tierhaltung und Pflanzenbau in der Landwirtschaft. Es berücksichtigt die C-, N- und Energieflüsse in Tierhaltungs- und WirtschaftsdüngerManagementsystemen. Es liefert weitere Emissionsinformationen an RAUMIS.

\subsubsection{Modellentwicklungen und Simulationskonzepte}

Horst Gömann

\subsubsection{Landnutzungsmodellierung mit dem Land Use Scanner}

Roland Goetzke und Jana Hoymann

Für die Entwicklung des Landnutzungsszenarios 2030 für Deutschland wird das GIS-basierte Simulationsmodell Land Use Scanner (LUS) verwendet (Hilferink und Rietveld 1999; Koomen et al. 2011). Das Modell wurde 1997 an der Vrijen Universiteit 
Amsterdam entwickelt und seitdem weiterentwickelt. Es wird insbesondere in den Niederlanden bei der Beantwortung zahlreicher politikrelevanter Fragestellungen angewendet (Scholten et al. 1999; Schotten et al. 2001; Koomen et al. 2008; Jacobs et al. 2011). In abgewandelter Form wird es derzeit auf europäischer Ebene genutzt, um Auswirkungen politischer Entscheidungen zu analysieren (Lavalle et al. 2011). Für die hier vorliegende Modellierung von Landnutzungsänderungen in Deutschland wurde der LUS technisch angepasst, um die gestellten Anforderungen hinsichtlich der verwendeten Daten und der Implementierung unterschiedlicher Landnutzungsstrategien zu erfüllen.

Der LUS ist ein operationelles, räumlich explizit arbeitendes Modell, welches mit einem Optimierungsalgorithmus die Nachfrage nach Fläche auf dafür geeignete Rasterzellen verteilt (Abb. 4.2). Die Nachfrage wird in externen Modellen für Regionen, z. B. Landkreise, bestimmt. Die Identifikation geeigneter Rasterzellen geschieht mit Hilfe von Eignungskarten, in denen Standortfaktoren wie aktuelle Landnutzung, physische

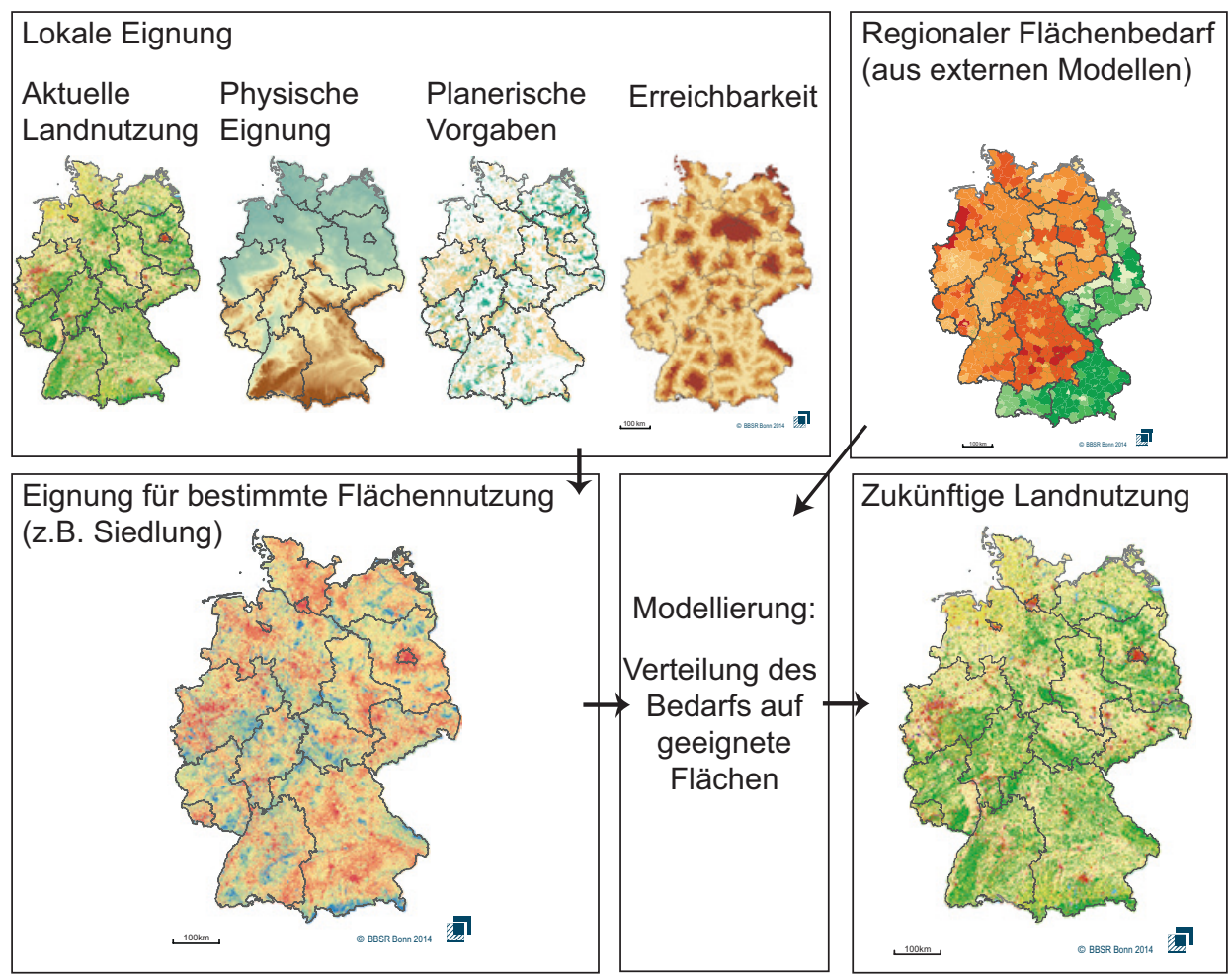

Datenbasis: Laufende Raumbeobachtung des BBSR,Corine Land Cover 2006, DGM: NASA 2000, Schutzgebiete: BfN 2011; Geometrische Grundlage: BKG 31.12.2008

Abb. 4.2 Funktionsprinzip des Land Use Scanners 
Gegebenheiten, planerische Festlegungen oder Erreichbarkeit von Infrastrukturen zusammengefasst werden. Die einzelnen Landnutzungsarten stehen dabei in Konkurrenz zueinander.

Das Modell hat einige grundlegende Eigenschaften: Es arbeitet rasterbasiert mit einer Auflösung von derzeit $100 \times 100$ m. In das Modell kann eine beliebige Anzahl Landnutzungsarten, in Abhängigkeit von der zur Verfügung stehenden Datenbasis, integriert werden. Damit betrachtet es nicht ausschließlich urbane Landnutzungen. Im LUS wird jeder Rasterzelle genau eine Landnutzung zugeordnet. Die Ergebnisse des Modells sind bei gleicher Parametereinstellung reproduzierbar. Festgesetzte Planungen können im LUS integriert und bei der Simulation berücksichtigt werden. Zudem liefert das Modell Ergebnisse für aufeinanderfolgende Zeitschritte. In der hier genutzten Version wird für jeden 5-Jahres-Zeitraum eine Landnutzungskarte erzeugt. Der Allokationsalgorithmus optimiert die Verortung der Landnutzungsarten so, dass die Summe der Eignungswerte der von Landnutzungswandel betroffenen Zellen maximiert wird. Drei Randbedingungen müssen dabei erfüllt werden (Koomen et al. 2011, S. 14 ff.):

- Die Fläche, die in einer Zelle verortet wird, darf nicht negativ sein,

- es kann nur 1 ha Fläche pro Zelle verortet werden und

- der Flächenanteil einer Landnutzungsart, die in einer Region verortet wird, muss zwischen dem angegebenen minimalen und maximalen Raumanspruch der Nachfrage nach dieser Landnutzungsart liegen. Eine mathematische Formulierung dieses Allokationsalgorithmus ist in Koomen et al. (2011, S. 14 ff.) zu finden.

Die Nachfrage nach Siedlungs- und Verkehrsfläche wird in einem externen Modell berechnet. Hierfür wurde das umweltökonomische Modell Panta Rhei Regio (PRR) verwendet (BMVBS 2011), um die Flächeninanspruchnahme durch Siedlung und Verkehr bis $2030 \mathrm{zu}$ projizieren. Dies geschieht differenziert nach Nutzungsarten auf Ebene der Kreise und kreisfreien Städte. Dabei schätzt das Modell die Flächenentwicklung ökonometrisch anhand vergangener Flächentrends und sozioökonomischer Entwicklungen. Die Ergebnisse dieser Projektion werden ausführlich in Hoymann und Goetzke (2014) vorgestellt.

Das Ziel der Modellierung mit dem LUS ist es, das räumliche Muster künftiger Flächeninanspruchnahme auch unterhalb der Aggregationsstufe Landkreis und kreisfreie Stadt abzubilden und zu analysieren. Hierfür wird die auf Kreisebene vorliegende Siedlungs- und Verkehrsflächenprojektion auf einen flächenscharfen Landnutzungsdatensatz übertragen. Als Grundlage für ein solches räumliches Simulationsmodell ist ein Datensatz notwendig, der die Flächennutzung in Deutschland räumlich möglichst detailliert repräsentiert. Ein Überblick und Vergleich aktueller Daten wurde von Hoymann (2013) vorgenommen. Im vorgestellten Szenario wird ein kombinierter Datensatz aus dem Landbedeckungsmodell Deutschlands (LBM-DE) des Bundesamtes für Kartographie und Geodäsie (BKG) und dem Copernicus Urban Atlas verwendet, der zudem durch Informationen aus dem ATKIS Basis-DLM ergänzt wurde. Durch die Kombination dieser 
Datensätze wurde eine optimale räumliche und thematische Repräsentation der Flächennutzung erreicht, die jeweils mit den einzelnen Datensätzen nicht möglich gewesen wäre. Ein ähnliches Verfahren zur Erstellung eines verfeinerten Landnutzungsdatensatzes auf europäischer Skala ist bei Batista e Silva et al. (2013) dokumentiert. ${ }^{2}$ Das Aggregieren der großmaßstäbigen Informationen aus LBM-DE und Urban Atlas auf 1 ha-Rasterzellen führt dazu, dass kleine und linienhafte Elemente in diesem Datensatz unterschätzt werden. So ist der Anteil der Verkehrsflächen im kombinierten Landnutzungsdatensatz kleiner als in der Flächenstatistik, die im Modell PRR verwendet wurde. Ein bedeutender Teil der Verkehrsflächen entfällt nach dem Aggregieren auf die anderen (flächenhaften) urbanen und landwirtschaftlichen Landnutzungsklassen. Dies muss bei der Interpretation der Ergebnisse und Übergabe an die Modelle RAUMIS sowie FoBeSiMo berücksichtigt werden.

In die Eignungskarten fließen neben der Flächennutzung zahlreiche weitere räumliche Informationen ein, die einen potenziellen Einfluss auf die zukünftige Änderung der Flächennutzung haben. Dazu zählen Daten zu Geomorphologie, Erreichbarkeit von verkehrlicher und sozialer Infrastruktur oder planerische Vorgaben aus Regionalplänen und Fachplanungen (vgl. Abb. 4.2). Die Eingangsdaten und die Methodik zur Berechnung der Eignungskarten werden detailliert in Hoymann und Goetzke (2014) beschrieben.

Alle räumlichen Einzelinformationen werden gewichtet und in jeweils einer Eignungskarte für jede zu modellierende Landnutzungsart zusammengefasst. Auf diese Weise liegen dem Landnutzungsmodell für jede Rasterzelle Informationen zur lokalen Eignung für jede Landnutzungsart vor. Landnutzungsänderungen werden vom LUS überall dort vorgenommen, wo eine andere Landnutzung stärker ,geeignet“ ist als die derzeit vorhandene, die entsprechende regionale Nachfrage gegeben ist und bestimmte andere im Modell definierte Regeln zutreffen. Dieses Regelwerk beinhaltet Parameter, die bestimmen, ob sich die modellinterne „Investition“ zur Aktivierung einer Fläche für eine neue Nutzung lohnt und nach welchem Zeitraum sich diese amortisiert. So wird ein Wald erst nach vielen Jahrzehnten Gewinn abwerfen und dementsprechend nicht schon nach wenigen Jahren für eine neue Landnutzung in Betracht gezogen. Ebenso sind Siedlungs- und Verkehrsflächen sehr persistent, da ihr Bau mit hohen Investitionskosten verbunden ist.

\subsubsection{Modellierung der landwirtschaftlichen Landnutzung mit RAUMIS}

Martin Henseler, Horst Gömann und Peter Kreins

Das Regionalisierte Agrar- und Umweltinformationssystem für Deutschland (RAUMIS) ist ein agrarökonomisches Optimierungsmodell, das die landwirtschaftliche Produktion, Einkommen und Umweltwirkungen in Deutschland auf regionaler Ebene abbildet. RAUMIS umfasst den gesamten Pflanzenbau (z. B. Marktfrüchte, Futterpflanzen,

\footnotetext{
${ }^{2}$ Dort wurde als Datenbasis der europaweit einheitliche, aber in seiner räumlichen Ausprägung weniger detaillierte CORINE Land Cover Datensatz verwendet.
} 
Grünland und Energiepflanzen) sowie die Tierhaltung. Das Modell wurde in den 1990er-Jahren entwickelt und seitdem für die Politikberatung und Forschungsaufgaben verwendet und weiterentwickelt. RAUMIS wird insbesondere zur modellgestützten Politikfolgenabschätzung der Agrar- und Umweltpolitik auf die landwirtschaftliche Produktion, Landnutzung, Einkommen sowie Umweltwirkungen eingesetzt.

Sogenannte Regionshöfe repräsentieren die landwirtschaftliche Produktion auf der Ebene von Landkreisen. Die Regionshöfe umfassen sämtliche Produktionsverfahren und -faktoren der Landkreise und verfolgen das Ziel der Gewinnmaximierung. Gewinnmaximierung wird im Rahmen eines linearen Programmierungsansatzes abgebildet, der sämtliche landwirtschaftliche Produktionsverfahren umfasst. Im Zuge der Kalibrierung der Produktionsumfänge auf ein Basisjahr und Anwendung der Methode der Positiven Mathematischen Programmierung (PMP; Cypris 2000) wird eine nichtlineare Zielfunktion abgeleitet, durch die im Wesentlichen steigende Grenzkosten der Substitution zwischen den Produktionsverfahren simuliert werden. Bei sich ändernden politischen und/oder ökonomischen Rahmenbedingungen passen die Regionshöfe entsprechend der nichtlinearen Zielfunktion ihr Produktionsprogramm an, um ihren Gewinn unter den neuen Rahmenbedingungen zu maximieren. Ausgehend vom Basisjahr wird die landwirtschaftliche Produktion für ein Zieljahr projiziert, das als Referenzprojektion für komparative statische Analysen dient (vgl. Henrichsmeyer et al. 1996).

Die RAUMIS-Modelldatenbasis ist konsistent mit der Landwirtschaftlichen Gesamtrechnung des Bundesministeriums für Ernährung und Landwirtschaft (BMEL) und den praxisüblichen landwirtschaftlichen Produktionsverfahren nach Anbauempfehlungen, z. B. des Kuratoriums für Technik und Bauwesen in der Landwirtschaft (KTBL). Die Produktionsumfänge und Erträge basieren auf den Regionalstatistiken der Landkreise und Gemeinden der Statistischen Landesämter und repräsentieren somit die regionalspezifischen landwirtschaftlichen Produktionsverfahren und -ausrichtungen (vgl. Weingarten 1995; Henrichsmeyer et al. 1996; Cypris und Kreins 1998; Cypris 2000).

Ursprünglich adressierte RAUMIS im Bereich der Politikfolgenabschätzung vorwiegend die Auswirkungen und Bewertung der Instrumente der Gemeinsamen Agrarpolitik (GAP) und deren Reformen: die Entkoppelung von Produktionsprämien, die Einführung und Reformen von Agrar- und Umweltprogrammen und Reformen der Marktordnungen (z. B. die Produktionsquoten von Zucker, Milch). Auch waren die Entwicklung der Agrarmärkte (z. B. Preiswirkungen) sowie der Einfluss des globalen Wandels auf die Agrarmärkte von Interesse (vgl. Bertelsmeier et al. 2002; Kreins et al. 2002; Gömann et al. 2003; Isermeyer et al. 2006; Kreins und Gömann 2008; Offermann et al. 2016).

Im Bereich „Umweltwirkungen“ wurde RAUMIS bisher im Wesentlichen in zwei Forschungsschwerpunkten eingesetzt: zur Analyse von Pflanzenschutzmittelanwendungen (vgl. Gömann et al. 2002; Sieber 2003; Julius et al. 2003; Sieber et al. 2010) und zur Quantifizierung landwirtschaftlicher Nährstoffeinträge und ihrer Umweltwirkungen in Bezug auf die Gewässerqualität. Im Forschungsbereich „Nährstoffe und Gewässerschutz“ wurden im Rahmen von mehreren interdisziplinären Verbundprojekten Schnittstellen zu 
hydrologischen Modellen entwickelt (vgl. Weingarten und Kreins 2003; Gömann et al. 2004, 2011; Kunkel et al. 2005; Hirt et al. 2008; Kuhn et al. 2008; Tetzlaff et al. 2007, 2008; Wendland et al. 2009; Kuhr et al. 2013).

Zur Analyse der Auswirkungen der Förderung erneuerbarer Energien und dem zunehmenden Anbau von Silomais als nachwachsendem Rohstoff zur Erzeugung von Biogas wurde RAUMIS um das Verfahren Energiemais erweitert (vgl. Gömann et al. 2007a, b, 2010; Gömann und Kreins 2012).

Im Bereich der THG-Bilanzierung liefert RAUMIS die Aktivitätsdaten zur Berechnung der Emissionen aus der landwirtschaftlichen Pflanzen- und Tierproduktion, die in der THG-Emissionsberichterstattung ausgewiesen werden (vgl. Rösemann et al. 2015; Haenel et al. 2016).

Zur Anwendung auf die Fragestellungen des Projekts CC-LandStraD und zur Implementierung in dessen Modellverbund wurde RAUMIS um verschiedene Aktivitäten und Indikatoren erweitert. Der Bereich Politikfolgenabschätzung wurde um die Simulation von Politikinstrumenten zur THG-Emissionsvermeidung erweitert. Die Maßnahme der Emissionssteuern wurde in RAUMIS implementiert und gibt als Klimavermeidungspolitik einen Anreiz, Mitigationsmaßnahmen umzusetzen (vgl. Schuler et al. 2014; Röder et al. 2015). Im Bereich Umweltwirkungen wurde RAUMIS um die Indikatoren der Stickstoffflächenbilanz und des Stickstoffgehalts im Sickerwasser erweitert. Beide Umweltindikatoren ermöglichen die Analyse der Umweltwirkung von Klimaschutzpolitik auf die Gewässerqualität. Des Weiteren wurde die Wiedervernässung von landwirtschaftlich genutzten organischen Böden als Aktivität zur Reduzierung von THG-Emissionen modelliert (vgl. Schuler et al. 2014; Röder et al. 2015).

Der Bereich „Erneuerbare Energien“ wurde um die Aktivitäten der Paludikultur (Schilfanbau auf Nieder- und Anmoorstandorten außerhalb der Schutzgebietskulisse) als alternative Biogassubstratpflanze erweitert (vgl. Schuler et al. 2014; Röder et al. 2015). Als weitere Produktionsaktivität für erneuerbare Energien wurde der Anbau von Kurzumtriebsgehölzen in RAUMIS implementiert (vgl. Henseler et al. 2015; Röder et al. 2015).

Im Bereich „THG-Bilanzierung“ wurde RAUMIS um mehrere Indikatoren erweitert. Diese sind Modellschnittstellen zu Emissionsmodellen und ermöglichen die Simulation von THG-Emissionen aus landwirtschaftlicher Produktion. Die THG-Emissionen sind regional und nach Bodentyp (Mineralböden, organische Böden) differenziert. Des Weiteren werden THG-Emissionen aus der Tierhaltung abgebildet (vgl. Henseler und Dechow 2014; Röder et al. 2015).

Im CC-LandStraD-Modellverbund nimmt RAUMIS durch die Schnittstellen zu fast allen Teilmodellen eine zentrale Position ein. Über die Modellschnittstellen zu CAPRI, Land Use Scanner und FoBeSiMo erhält RAUMIS die Informationen zu Entwicklungen der Agrarpreise und Landnutzungsänderungen durch Siedlung, Verkehr und Forst. Über die anderen Teilmodelle erhält RAUMIS die Daten für biophysikalische Prozesse (z. B. zur THG-Emissionsberechnung aus Böden von MODE und aus der Tierhaltung von GAS-EM). 
Durch die Erweiterungen um neue Aktivitäten und Indikatoren sowie die Kopplung zu den CC-LandStraD-Teilmodellen werden verschiedene Einzelmaßnahmen sowie Strategien simuliert. Die Modellergebnisse werden regional und für gesamt Deutschland ausgewiesen und analysiert. Somit ermöglicht RAUMIS eine differenzierte Analyse der Auswirkungen simulierter Klimaschutzmaßnahmen auf die landwirtschaftliche Produktion, Einkommen, Umwelt einschließlich der Höhe der THG-Emissionen.

\subsubsection{THG-Modellierung - u. a. MODE}

\section{René Dechow}

\section{Lachgas-Emissionen mineralischer Böden}

Landnutzungsbedingte THG-Emissionen werden im Rahmen der Klimaberichterstattung durch Emissionsfaktoren beschrieben. Dies gilt für direkte Lachgas-Emissionen mineralischer Böden als auch für THG-Emissionen organischer Böden. Beide Quellen zusammengenommen machen etwa $90 \%$ landnutzungsbedingter THG-Emissionen Deutschlands aus. Emissionsfaktoransätze basieren auf der Multiplikation von Emissionsfaktoren mit den jeweiligen Aktivitätsdaten der Produktionsverfahren.

Im Fall direkter Lachgas-Emissionen mineralischer Böden sind die Aktivitätsdaten die Quellen reaktiven Stickstoffs, der den Agrarökosystemen durch Düngung, Pflanzenresiduen, Stickstofffixierung und Mineralisierung des Bodenhumus hinzugefügt wird. Der Emissionsfaktor ist eine Proportionalitätskonstante, die das Verhältnis von zugeführtem reaktiven Stickstoff und der Lachgas-Emission beschreibt. Aktuell basiert die Berichterstattung auf dem TIER 1 Ansatz, das heißt, die hier verwendeten Emissionsfaktoren wurden anhand weltweiter Messwertdatensätze ermittelt und sind im Rahmen der Klimaberichterstattung in allen Staaten anwendbar, die im Rahmen internationaler Abkommen (Kyoto-Protokoll, UNFCC) Treibhausgasinventare erstellen. Der Emissionsfaktor für die Bildung direkter THG-Emissionen aus mineralischer und organischer Düngung beträgt beispielsweise $1 \%$, was bedeutet, dass weltweit etwa $1 \%$ des über Düngung zugeführten Stickstoffs in Agroökosystemen als Lachgas emittiert wird.

Der Tier 1 Ansatz vernachlässigt die regionalspezifischen Bedingungen, die zum Beispiel bei der Bildung von Lachgas in Böden eine bedeutende Rolle spielen. Im Fall von direkten Lachgas-Emissionen ist bekannt, dass diese durch mikrobielle Stoffumsätze in Böden gebildet werden. Ein wichtiger Reaktionspfad ist die Denitrifikation, bei der Nitrat zu atmosphärischen Stickstoff reduziert wird und Lachgas als Zwischenprodukt entsteht. Mikroorganismen kompensieren Sauerstoffmangel über die Denitrifikation, diese ist deshalb vor allem dann zu beobachten, wenn hohe Wassergehalte im Boden den Gasaustausch zwischen Bodenporenraum und Atmosphäre behindern. Ebenso kann eine verstärkte Bodenatmung durch Zugabe leicht abbaubarer organischer Substanzen zu Sauerstoffmangel im Boden führen. Zahlreiche Studien haben gezeigt, dass hohe $\mathrm{N}_{2} \mathrm{O}-$ Emissionsraten bevorzugt bei hohen Wassergehalten auftreten, vorausgesetzt, Nitrat als Ausgangsstoff der Denitrifikation ist kein limitierender Faktor. Darüber hinaus wird oft von hohen Emissionen im Winter, nach dem Auftauen gefrorenen Bodenwassers berichtet. $\mathrm{Zu}$ diesem Zeitpunkt ist der Luftaustausch zwischen Bodenporenraum und 
Atmosphäre gehemmt, zusätzlich liefern Residuen von durch Bodenfrost abgetöteten Mikroorganismen und die Zerstörung von Bodenaggregaten leicht abbaubare organische Bestandteile, was die Bodenrespiration ankurbelt und den Sauerstoffmangel weiter verschärft. Neben den klimatischen Bedingungen sind es insbesondere Bodeneigenschaften, welche das Wasserspeichervermögen und den Anteil des luftgefüllten Porenraums kontrollieren und so die Lachgasbildung steuern.

Um regionale Bedingungen in die Berechnung von Lachgas-Emissionen zu integrieren und so eine räumlich explizite integrierte Modellierung zu ermöglichen, wurden regionalspezifische Emissionsfaktoren mit MODE erstellt und in RAUMIS verwendet (Henseler und Dechow 2014). Dabei entspricht die räumliche Auflösung dieser Emissionsfaktoren der des agrarökonomischen Modells RAUMIS. Die gesamtdeutschen LachgasEmissionen landwirtschaftlicher Böden resultieren als Summe der Lachgas-Emissionen in allen Regionen, in denen die Anbauverfahren jeweils mit den regionalspezifischen Emissionsfaktoren multipliziert wurden.

MODE ist ein auf „fuzzy logic“ basierendes empirisches Modell, das an Datensätzen experimentell bestimmter annueller Lachgas-Emissionen trainiert und validiert wurde (Dechow und Freibauer 2011). In MODE werden die Landnutzung Acker und Grünland unterschieden. Im Rahmen einer automatisierten Faktorensuche wurden für beide Ökosystemtypen die Faktorenkombinationen mit dem größten Anteil erklärter Variabilität jährlicher Lachgasflüsse ermittelt. Dies waren für Ackerstandorte die Temperatur im Winter, der Niederschlag im Herbst, der Sandgehalt des Bodens, die Menge an appliziertem Stickstoff und die Fruchtartenkategorie (Getreide, Hackfrüchte, Gemüse, Raps, Brache, alle anderen). Anhand des erstellten Modells zeigte sich, dass die annuellen Lachgas-Emissionen negativ mit der mittleren Wintertemperatur und den Sandgehalten des Bodens aber positiv mit den Niederschlagssummen im Herbst korrelieren. Saisonaler Niederschlag und Sandgehalte sind Indikatoren für die Bodenwassergehalte und den luftgefüllten Porenraum eines Ackerstandortes. Die negative Korrelation von Wintertemperatur und Lachgas-Emission deutet den hohen Einfluss von durch FrostTau-Zyklen erzeugten Emissionsspitzen an. Das Verhältnis von emittierter LachgasEmission und zugeführtem reaktiven Stickstoff wird wesentlich durch die Kombination der klimatischen Faktoren und Bodeneigenschaften bestimmt. Lachgas-Emissionen aus Grünland können am besten über die Menge des zugeführten reaktiven Stickstoffs, die Wintertemperatur und den $\mathrm{pH}$-Wert des Bodens erklärt werden. Zudem treten höhere Emissionen bei Weidehaltung auf.

\section{THG-Emissionen organischer Böden}

Genutzte organische Böden emittieren Lachgas, Methan und Kohlendioxid. Wie die Bezeichnung schon suggeriert, zeichnen sich organische Böden durch hohe Mengen organischer Substanzen im Boden aus, die aufgrund ungünstiger Lebensbedingungen für die Bodenfauna kaum abgebaut wurden. Meist ist hier Sauerstoffmangel im Boden, verursacht durch hohe Grundwasserstände, verantwortlich. Landnutzungsintensität und die Art angebauter Kulturen sind auf bestimmte bodenhydrologische 
Bedingungen angewiesen, die über meliorative Maßnahmen hergestellt werden. In der Regel unterscheiden sich die mittleren Grundwasserstände verschiedener Nutzungstypen voneinander, wobei Ackernutzung die höchsten Grundwasserflurabstände aufweist, gefolgt von Grünland (je nach Nutzungsintensität) und Wald. Wird über meliorative Maßnahmen der Grundwasserstand langfristig oder periodisch abgesenkt, verbessern sich die Bedingungen für den Abbau organischer Bodensubstanz, und das Potenzial für die Bildung der Treibhausgase Lachgas und Kohlendioxid erhöht sich. Die Zusammenhänge zwischen Nutzungstyp, Grundwasserregulierung und THG-Emission werden im Rahmen der Klimaberichterstattung über nutzungsspezifische Emissionsfaktoren abgebildet, während die Aktivitätsdaten über die Fläche organischer Böden pro Nutzungstyp beschrieben werden.

Grundwasserstände organischer Böden werden nicht allein über die Nutzung geprägt. Die klimatische Wasserbilanz des Standorts, die Verteilung der Landnutzung im Umfeld, das Einzugsgebiet oder die relative Höhe der jeweiligen Fläche zur Umgebung sind weitere Faktoren, die als Indikatoren für die jeweiligen Grundwasserstände und damit auch die Höhe der Kohlendioxid und Lachgas-Emissionen verwendet werden können (Bechtold et al. 2014). Im Rahmen von CC-LandStraD wurden regionale landnutzungsspezifische Emissionsfaktoren für die Treibhausgase Kohlendioxid, Lachgas und Methan entwickelt, wobei die Differenzierung dieser Emissionsfaktoren im Wesentlichen auf die regionale Verteilung nutzungsspezifischer Grundwasserstände zurückzuführen ist. Ana$\log$ zur Erstellung regionaler Emissionsfaktoren für Lachgas-Emissionen mineralischer Böden basieren die Emissionsfaktoren für organische Böden auf einer Regionalisierung von THG-Modellen.

Das empirische Modell MODE wurde für die Abbildung von Lachgas-Emissionen organischer Böden modifiziert. Ähnlich wie bei der Modellierung von LachgasEmissionen mineralischer Standorte, wurde zunächst ein Datensatz zu annuellen Lachgas-Emissionen organischer Böden zusammengestellt, an dem MODE trainiert und evaluiert wurde (Leppelt et al. 2014). Die entscheidenden Steuergrößen variieren in Abhängigkeit vom Landnutzungstyp. So erwiesen sich beispielsweise mittlere Grundwasserstände, Jahresniederschläge und der $\mathrm{pH}$-Wert des Bodens als relevant für die Abbildung von Lachgas-Emissionen aus organischen Böden unter Ackernutzung. Im Fall von Grünlandnutzung war es die Menge zugeführten reaktiven Stickstoffs, die Temperatur im Winter und der akkumulierte Niederschlag im Herbst.

Messungen annueller $\mathrm{CO}_{2}$-Emissionen wurden herangezogen, um einen Ansatz zur Abschätzung annueller $\mathrm{CO}_{2}$-Emissionen $\mathrm{zu}$ parametrisieren (Drösler et al. 2013). Die annuelle $\mathrm{CO}_{2}$-Emission ergibt sich dabei aus Netto- $\mathrm{CO}_{2}$-Fluss zwischen Ökosystem und Atmosphäre und der Bilanz aus Kohlenstoffzufuhr und -abfuhr durch organische Düngung und Ernte, wobei die $\mathrm{CO}_{2}$-Bilanz abhängig vom Wasserstand ist. Der in dem verwendeten Datensatz zu beobachtende Einfluss des mittleren Grundwasserstands auf den Ertrag wurde über eine nichtlineare Regression abgebildet. Parameterunsicherheiten wurden über die Verwendung von Bayesscher Kalibrierung quantifiziert. 
Methan-Emissionen organischer Böden ergeben sich aus der Bilanz von Methanbildung unter anaeroben Bedingungen und der Methankonsumption, die aerobe Bedingungen erfordert. Der jeweilige Wasserstand steuert die Mächtigkeit der aeroben Bodenzone, in der aufsteigendes Methan durch Mikroorganismen abgebaut wird. Der im Rahmen von CC-LandStraD entwickelte Modellansatz beschreibt Methanbildung und Methankonsumption als chemische Reaktionen erster Ordnung. Dabei wird die Reaktionsgeschwindigkeit über Wasserstand, Temperatur und Konzentration des Ausgangssubstrats modifiziert.

\subsubsection{Entwicklung des Forstbetrieblichen Simulationsmodells FoBeSiMo}

Nils Ermisch, Hermann Englert, Margret Köthke und Peter Elsasser

Für die Analyse der Anpassungen von Forstbetrieben an veränderte Rahmenbedingungen wurde im Rahmen von CC-LandStraD das Forstbetriebliche Simulationsmodell (FoBeSiMo) entwickelt. Mit Hilfe von FoBeSiMo lassen sich Auswirkungen klimatisch bedingter Veränderungen von Produktionsbedingungen, Änderungen wirtschaftlicher Rahmenbedingungen sowie mögliche Reaktionen der Forstbetriebe sowohl auf naturale als auch wirtschaftliche Ergebnisse in der Forstwirtschaft abbilden. Dabei sind in FoBeSiMo die regional unterschiedlichen Standortbedingungen in Deutschland berücksichtigt. Der Aufbau des Modells FoBeSiMo ist in Abb. 4.3 schematisch dargestellt und umfasst als drei zentrale Module 1) forstliche Modellbetriebe, 2) Entscheidungsregeln und 3) ein integriertes Simulationsmodul.

\section{Forstliche Modellbetriebe}

Zur Abbildung möglicher Anpassungsreaktionen unterschiedlicher Forstbetriebe wurden erstmalig für Deutschland 13 typische Forstbetriebe definiert, die jeweils die 13 Flächenbundesländer repräsentieren und damit den gesamten deutschen Wald. Die Beschreibung der Modellbetriebe in den einzelnen Modellzeitpunkten erfolgt über ein Datenmodell, das ausgewählte naturale und ökonomische Daten umfasst. Die Steuerung der Fortschreibung der Zustandsdaten im Zeitverlauf erfolgt über Datenschnittstellen, die auf exogene und endogene Steuerdaten zurückgreifen. Exogene Steuerdaten dienen zur Abbildung des außerbetrieblichen Entscheidungsumfelds (Markt- und Standortdaten). Endogene Steuerdaten dienen der dynamischen Abbildung des forstbetrieblichen Zustands bzw. forstbetrieblicher Entscheidungen (Bewirtschaftungs- und Bestandesdaten).

a) Marktdaten

Die unterstellten Marktdaten fußen auf Angaben aus dem Testbetriebsnetz-Forst (TBN-Forst) des Bundesministeriums für Ernährung und Landwirtschaft (teilweise veröffentlicht in BMEL 2013). Dazu wurden unter Berücksichtigung von 


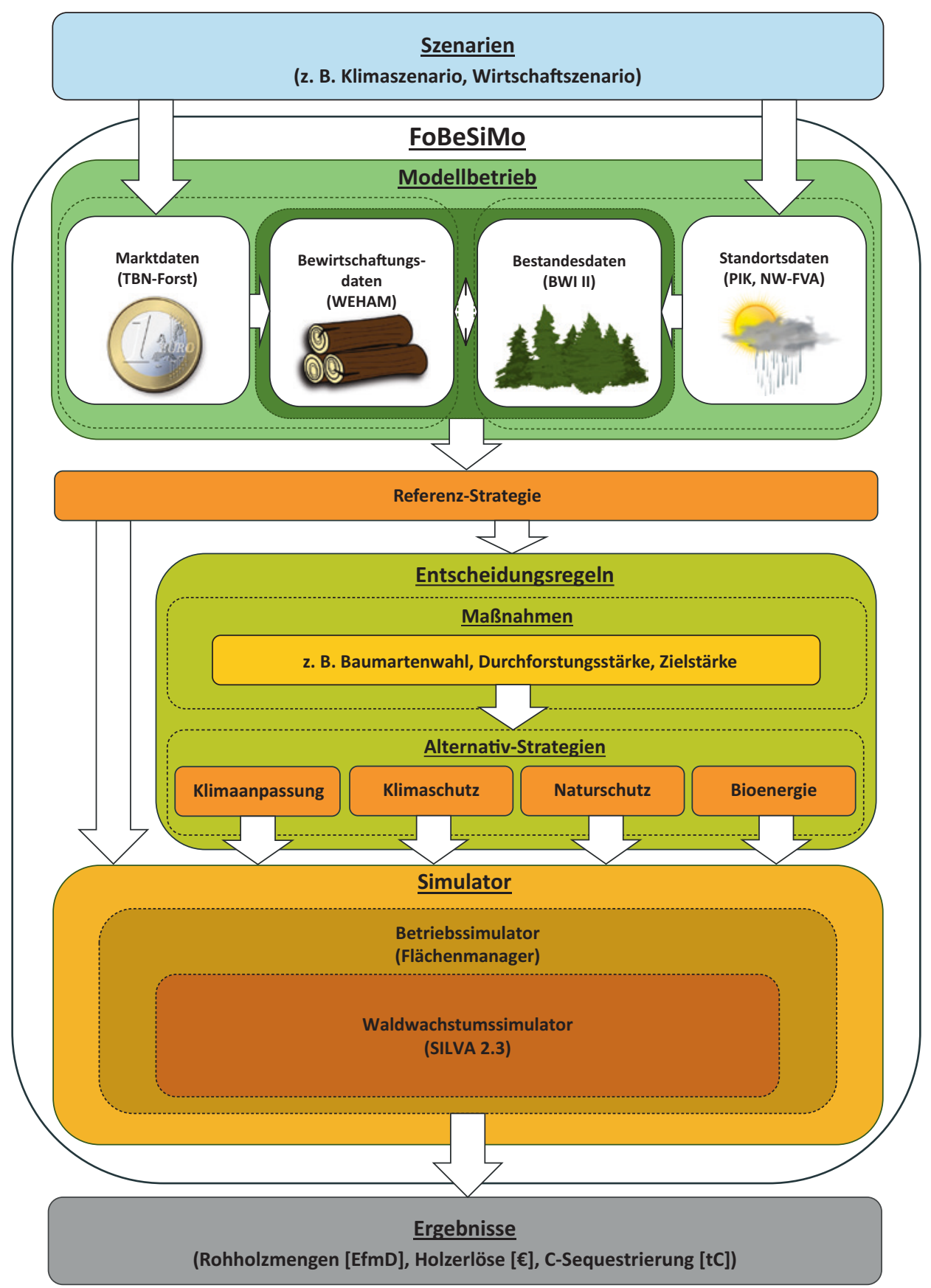

Abb. 4.3 Schematische Abbildung des forstbetrieblichen Simulationsmodells (FoBeSiMo) 
Selbstwerbermengen pro Holzartengruppe durchschnittliche erntekostenfreie Holzerlöse berechnet. Da die Erlös- und Kostengrößen aus Datenschutzgründen nicht eigentums- und länderspezifisch ausgewertet werden konnten, wurden die Eigentumsarten über alle Modellbetriebe nach ihren Einschlagsanteilen gewichtet und pro Holzartengruppen je ein gesamtsektoraler erntekostenfreier Holzerlös berechnet. Um Jahresschwankungen der Vergangenheit abzufangen, handelt es sich um durchschnittliche erntekostenfreie Holzerlöse der Jahre 2009 bis 2013. Zusätzlich wurden, basierend auf einer Literaturrecherche, unterschiedliche Kulturkosten veranschlagt, die zusätzlich nach Holzartengruppen und Verjüngungsart (Naturverjüngung oder Pflanzung) differenziert wurden. Ferner wurden Läuterungskosten berücksichtigt. Somit ist es möglich, pro Holzartengruppe einen Deckungsbeitrag I (DBI) ${ }^{3} \quad \mathrm{zu}$ berechnen. Des Weiteren wurden spezifische nicht verwertete Derbholzanteile (nvDHolzanteile) pro Holzartengruppe aus dem TBN-Forst abgeleitet. Die entsprechenden Annahmen bezüglich der unterstellten Marktdaten sind in Tab. 4.20 dargestellt. Als Kalkulationszins wurden die aktuell forstüblichen $1,5 \%$ p.a. angenommen.

b) Bewirtschaftungsdaten

Die für die Simulation der Bewirtschaftungsalternativen benötigten Annahmen wurden aus den Steuerdaten des Basisszenarios 2002 des WEHAM-Modells ${ }^{4}$ abgeleitet (Polley und Kroiher 2006 ). Es handelt sich dabei um die Steuerdaten $\mathrm{zu}$ zehn Bewirtschaftungsmaßnahmen, welche die Referenzbewirtschaftung und die Bewirtschaftungsziele der Alternativstrategien abbilden (vgl. Abschn. 4.6). Diese Bewirtschaftungsvorgaben nach WEHAM wurden für FoBeSiMo angepasst und mittels einer Analyse der aktuellen Waldbaurichtlinien der Bundesländer aktualisiert und überarbeitet. Da über den Körperschafts- und Privatwald keine ausreichenden Informationen vorlagen, wurden die Waldbewirtschaftungskonzepte des Staatswaldes im jeweiligen Bundesland auf diese beiden Eigentumsarten übertragen. Die Festlegung der Referenzbewirtschaftung bildet somit die Grundlage weiterer davon abweichender Bewirtschaftungsstrategien.

c) Bestandesdaten

Aus den Daten der Bundeswaldinventur (BWI) 2002 wurden für die vier Hauptbaumarten $^{5}$ Fichte, Kiefer, Buche und Eiche die dendrometrischen Werte je Bundesland berechnet (Thünen-Institut 2013). Diese bildeten die Eingangsdaten, um für jeden Modellbetrieb (bundeslandspezifische) Waldbestände zu modellieren.

\footnotetext{
${ }^{3}$ Der hier berechnete Deckungsbeitrag I entspricht dem waldbaulichen Deckungsbeitrag I, also dem erntekostenfreien Holzerlös (Verkaufserlös minus variable Holzerntekosten) abzüglich der Wiederbegründungskosten.

${ }^{4}$ WEHAM steht für WaldEntwicklungs- und HolzAufkommensModellierung.

${ }^{5}$ Im weiteren Verlauf werden die Hauptbaumarten (Eiche, Buche, Fichte und Kiefer) synonym für die vier Holzartengruppen (Eiche, Buche, Fichte und Kiefer) verwendet.
} 
Die Douglasie wird in der weiteren Analyse zwar als eigene Baumart geführt, ihr Wachstum sowie die ihr zugeschriebenen Kosten und Erlöse werden jedoch aus den entsprechenden Daten für Fichte übernommen. Die anderen Baumarten wurden den entsprechenden vier Hauptbaumarten (Fichte, Kiefer, Buche, Eiche) zugeschlagen und jeweils Durchschnitte der dendrometrischen Werte der Hauptbaumarten im Bundesland verwendet. Dies bedeutet, dass als Eingangswert der Kalibrierung pro Hauptbaumart ein durchschnittlicher Brusthöhendurchmesser für jeden Modellbetrieb verwendet wurde. Mit Hilfe des Waldwachstumssimulators SILVA wurden aus diesen Eingangswerten je Hauptbaumart und Modellbetrieb Klupplisten erstellt, die dann jeweils für eine Holzartengruppe Verwendung finden.

Für jedes Klimaszenario, jede Strategie und jeden Modellbetrieb wurde jeweils eine Wachstumskurve ab der Altersklasse 30 jeder Hauptbaumart modelliert. Somit liegen am Ende des Betrachtungszeitraumes für jeden Baum bzw. Bestand forstwirtschaftlich relevante Daten wie z. B. Durchmesser- und Höhenentwicklung vor. Alle weiteren Werte der Altersklassen unter den jeweiligen Bewirtschaftungsstrategien basieren auf diesen Wachstumskurven. Folglich ist keine dynamische Änderung von einer Strategie hin zu einer anderen im Zeitverlauf möglich. Zur Berechnung der genannten Wachstumskurven wurde der Einzelbaum-Wachstumssimulator SILVA (Biber et al. 2000) verwendet. Als Startaltersklasse wurde die Altersklasse 20-40 Jahre (im Mittel 30 Jahre) ausgewählt, da in der Altersklasse 0-20 Jahre die Bäume noch nicht alle die Brusthöhe (Messpunkt des Brusthöhendurchmessers) erreicht haben. Das beschriebene Vorgehen impliziert die Annahme, dass die Bewirtschaftung der jeweiligen Strategien zum Startzeitpunkt der Modellierung im Jahr 2015 bereits umgesetzt ist. Eine Übergangsphase vom heutigen Zustand hin zur jeweiligen Strategie wird nicht modelliert.

Da Risiko im Gegensatz zu Unsicherheit ein sowohl in der Folge als auch in der Eintrittswahrscheinlichkeit bekanntes Ereignis darstellt, ist es in Form altersspezifischer Überlebenswahrscheinlichkeiten nach Staupendahl (2011) für die vier Hauptbaumarten in der Modellierung berücksichtigt worden.

d) Standortdaten

Eine regionale Differenzierung für Deutschland erfolgte auf der Ebene der 13 Flächenbundesländer. In den Regionen wurden jeweils vier potenzielle Standorttypen unterschieden, die sich nach der aktuellen Bestockung der vier Hauptbaumarten zum Stand der BWI 2002 unterscheiden. Für die Beschreibung der Standorte verwendet SILVA neun Standortvariablen. ${ }^{6}$ Für die Standortdaten wurden in Zusammenarbeit mit der Nordwestdeutschen Forstlichen Versuchsanstalt und dem Potsdam-Institut für Klimafolgenforschung die einzelnen BWI-Stichprobenpunkte

\footnotetext{
${ }^{6}$ Je drei Standortvariablen zu Nährstoff $\left(\mathrm{NO}_{\mathrm{X}}, \mathrm{CO}_{2}\right.$, Nährstoffversorgung), Wärme (Anzahl der Tage mit Temperatur $>10^{\circ} \mathrm{C}$, Jahrestemperaturamplitude, Temperaturmittel Vegetationszeit) und Feuchtigkeit (Ariditätsindex Vegetationszeit, Bodenfrische, Niederschlag Vegetationszeit).
} 
mit der Bodenübersichtskarte (BGR 2004) und im Anschluss mit den Polygonen der Klimastationen verschnitten. Dies ermöglicht, temperaturabhängige Veränderungen der klimatischen und pedologischen Standortdaten regionalisiert zu berücksichtigen. Zur Herleitung von einheitlichen, bundeslandspezifischen Standortdaten ist aus den jeweiligen Mittelwerten der neun SILVA-Standortvariablen für jede der vier Hauptbaumarten der 13 Modellbetriebe ein „mittlerer Standort“ berechnet worden. Mit Hilfe des Waldwachstumssimulators SILVA wurden daraufhin baumarten- und standortspezifische Wachstumskurven der Alters-Höhenbeziehung berechnet. Diese lassen sich den ebenfalls ermittelten klimabedingten Wachstumskurven gegenüberstellen, um zwischen Baumart- und Standort- bzw. Klimaeffekten zu differenzieren.

2. Forstliche Entscheidungsregeln

Aus der Vielzahl möglicher Bewirtschaftungsmaßnahmen (vgl. Abschn. 3.3.2) wurden neun Einzelmaßnahmen ausgewählt (vgl. Abschn. 3.3.6), die die typischen Forstbetriebe im Rahmen dieser Studie ergreifen können, um sich veränderten Rahmenbedingungen anzupassen. Durch Variation der Ausprägung der Einzelmaßnahmen ist es möglich, verschiedene Strategien zu definieren, die sich in ihrer jeweiligen Umsetzung unterscheiden (vgl. Abschn. 4.6). Die Umsetzung der Einzelmaßnahmen innerhalb des Maßnahmenbündels richtet sich dabei nach dem jeweils verfolgten Strategieziel. Maßnahmen, ihre Varianten und die entsprechenden Strategien sind in Tab. 4.21 zusammengefasst.

3. Waldwachstums- und Betriebssimulator

Ausgehend von den dargestellten Start- und Steuergrößen lassen sich mit Hilfe von SILVA die Ausgangssituation eines Bestandes und die Standortbedingungen charakterisieren. In SILVA wird die Dynamik eines betrachteten Bestandes einzelbaumweise in 5-Jahres-Schritten von der Bestandesbegründung bis zum Generationenwechsel durch ein für den entsprechenden Standort initialisiertes Funktionensystem nachgebildet (Biber et al. 2000). Um die aus SILVA stammenden naturalen Ergebnisgrößen der Modellbetriebe ökonomisch bewerten zu können, wurde ein Betriebssimulator entwickelt. Der Betriebssimulator führt die von SILVA erzeugten Naturaldaten auf Bestandesebene zu Betriebsklassen zusammen. Die Betriebsklassen der 13 Modellbetriebe wurden auf Basis der Baumarten-, Flächenund Altersklassenverteilung der Bundesländer gemäß der BWI 2002 gebildet. Dieses Vorgehen ermöglicht es, einen Forstbetrieb aus unterschiedlichen (Haupt-)Baumarten auf unterschiedlichen Flächen in unterschiedlichen Altersklassen abzubilden und weiterführende ökonomische Analysen auf Betriebsebene durchführen zu können. Dadurch können die Holzmengen, Erlöse und Kosten sowie die Kohlenstoffmengen pro Modellbetrieb in den Simulationsperioden berechnet werden.

Zur Berechnung des jeweiligen Kohlenstoffgehaltes der vom Waldwachstumssimulator SILVA berechneten Holzvolumina und deren Substitutionspotenziale wurde auf einen Ansatz von Schweinle et al. (2018) zurückgegriffen. Dieser ermittelt auf Basis von Umrechnungsfaktoren aus der Literatur aus den vorliegenden Eingangsdaten den 
Kohlenstoffgehalt der drei Speicherkompartimente lebende Baumbiomasse (Derbholz und Nichtderbholz), Totholz und Holzprodukte sowie die jeweiligen Auswirkungen auf stoffliche und energetische Substitutionspotenziale. Holzprodukte werden dort nach Verwendungsbereichen unterteilt (Sägeholz, Platten, Papier), einschließlich der Berücksichtigung der jeweiligen Abbauraten. Veränderungen des Kohlenstoffgehaltes in Böden und Streu werden in diesem Modell nicht berücksichtigt.

\subsubsection{Modellierung der Stoffflüsse im Forst mit 4C}

Martin Gutsch, Petra Lasch-Born und Felicitas Suckow

Die Untersuchung der Auswirkungen von Landnutzungs- und Klimaänderungen auf den Stoffhaushalt der Waldbestände in Deutschland erfolgt mit Hilfe des prozessbasierten Waldwachstumsmodells 4C. Mit 4C werden sowohl der Stoffhaushalt als auch die forstliche Wuchsleistung von Wäldern unter sich ändernden Klimabedingungen und unter Bewirtschaftung analysiert (Lasch et al. 2005; Lasch-Born et al. 2015). Um flächendeckende Aussagen ableiten zu können, wurde das Modell 4C für 69.393 Waldbestände in Deutschland angewendet. Die Anzahl der Bestände ergab sich durch die Datengrundlage der Bundeswaldinventur (BWI ${ }^{2}$-Erhebung 2002), auf deren Basis die Charakterisierung der Bestandesausgangssituation erfolgte (BMELV 2005). Die Daten der Untersuchungsplots der BWI ${ }^{2}$ lieferten für jeden simulierten Waldbestand die Informationen „Baumart“ und „Alter“. Um die Initialisierung der Bestände für die Modellsimulation zu ermöglichen, mussten Vereinfachungen in der Bestandsstruktur vorgenommen werden. Berücksichtigung fanden nur die Baumartengruppen Fichte, Kiefer, Douglasie, Buche, Eiche ${ }^{7}$ und sonstige Laubhölzer niedriger Lebensdauer.

Des Weiteren wurden den Beständen die bundesland-, baumarten- und altersklassenspezifischen mittleren Bestandesparameter (Höhe, Bursthöhendurchmesser und Grundfläche) zugeordnet und für die Bestandesinitialisierung genutzt. Dadurch ist gesichert, dass die Aggregation der Bestände auf Kreis-, Landes- und Bundesebene den Erhebungen der BWI ${ }^{2}$ entspricht. Aus den Informationen, die durch die Verschneidung der Koordinaten jedes einzelnen Bestands mit der BÜK 1000 (BGR 2004) gewonnen wurden, wurde für jeden Bestand der entsprechende Boden initialisiert. Weiterhin wurde jedem Bestand die nächstliegende der insgesamt 1218 Klimastationen zugeordnet. Auf der Grundlage der zum Bestand gehörenden Waldfläche (CORINE Landnutzungsdatensatz; DLR-DFD 2004; CORINE Land Cover [CLC] 2000) werden die Ergebnisse der Simulationen auf die drei o.g. Verwaltungsebenen aggregiert. Diese Vorgehensweise ermöglichte eine flächige Simulation des Gesamtwaldes, ohne jedoch exakte Aussagen zu konkreten Waldbeständen machen zu können.

\footnotetext{
${ }^{7}$ Keine Unterscheidung von Traubeneiche und Stieleiche.
} 


\subsection{Einordnung der verwendeten Klimaszenarien}

Frank Wechsung, Tobias Conradt, Pia Gottschalk und Martin Gutsch

\section{Zusammenfassung}

Die Szenarienstudie CC-LandStraD bezieht sich auf Vermeidungsstrategien des Klimawandels. Klimawandel und seine Auswirkungen werden exemplarisch betrachtet. Die innerhalb des Projektes betrachteten Klimaszenarien und Folgenbetrachtungen liefern keine konkreten Begründungen, sondern illustrieren noch einmal den Anlass. In diesem Kapitel wird das genutzte Klimaszenario motiviert und eingeordnet. Ausgangspunkt bildet ein zusätzlicher globaler Strahlungsantrieb von $8,5 \mathrm{~W} / \mathrm{m}^{2}$ bis zum Jahr 2100 . Die daraus mit globalen Zirkulationsmodellen für Deutschland ermittelte Erwärmung wurde mit dem Szenariengenerator STAR zu einem Klimaszenario komplettiert. Zur Plausibilisierung und Einordnung des STAR-Szenarios wurden Ergebnisse der Projekte ISI-MIP und CORDEX-Europe herangezogen.

Das Klima in Deutschland hat sich in den letzten hundert Jahren deutlich erwärmt. Die Erwärmung ist Teil einer globalen Entwicklung und kann mit dem Anstieg der Emissionen von Treibhausgasen wie $\mathrm{CO}_{2}$, Methan oder Stickoxiden erklärt werden. Die Erwärmung der letzten Jahrzehnte kennt sowohl Phasen eines besonders starken als auch eines schwächeren Temperaturanstiegs. Ein besonders rasanter Temperaturanstieg war global in den 1980er- und 1990er-Jahren zu verzeichnen. Dieser hat sich zu Beginn des 21. Jahrhunderts jedoch nicht in dieser Geschwindigkeit fortgesetzt, was zu einer intensiven Diskussion über das Bestehen einer Erwärmungspause und seiner Gründe geführt hat (Flato et al. 2013). Die Entwicklung der mittleren Temperatur in Deutschland seit 1881 ist in Abb. 4.4 dargestellt. Auch hier sind Phasen deutlicherer und schwächerer Änderungen erkennbar.

Mit oder ohne Erwärmungspause muss nach dem bisherigen Erkenntnisstand davon ausgegangen werden, dass sich langfristig die Zunahme der Jahresmitteltemperatur in dem Maße fortsetzt, wie auch die THG-Konzentrationen weiter ansteigen. Die in dieser Formulierung mit zum Ausdruck kommende Unsicherheit speist sich aus unterschiedlichen Quellen. Alle Projektionen zum aktuellen Klima gehen von (in den gewohnten Schwankungsbereichen) stabilen kosmischen Randbedingungen (Sonnenaktivität) und einer weitgehend unveränderten vulkanischen Aktivität der Erde aus. Die Unsicherheit resultiert daneben aus der Vielfalt möglicher Emissionspfade und den objektiven und subjektiven Begrenzungen von Klimamodellen. Die Vielfalt möglicher Emissionspfade wird kategorisiert anhand der durch sie hervorgerufenen Störung des Strahlungsantriebes (,,radiative forcing“, RF, siehe unten). Unterschiedliche Entwicklungspfade sind vorstellbar. Sie hängen sowohl von den technologischen Möglichkeiten als auch dem politischen Willen für den Ausstieg aus der Energieerzeugung durch Verbrennung fossiler Energieträger ab.

Die Klimamodelle fassen den gegenwärtigen Erkenntnisstand zur Klimasensitivität auf Änderungen des Strahlungsantriebes und seine regionale Manifestierung zusammen. Stabile Änderungssignale sind aus diesen Modellen gegenwärtig nur durch starke zeitliche 


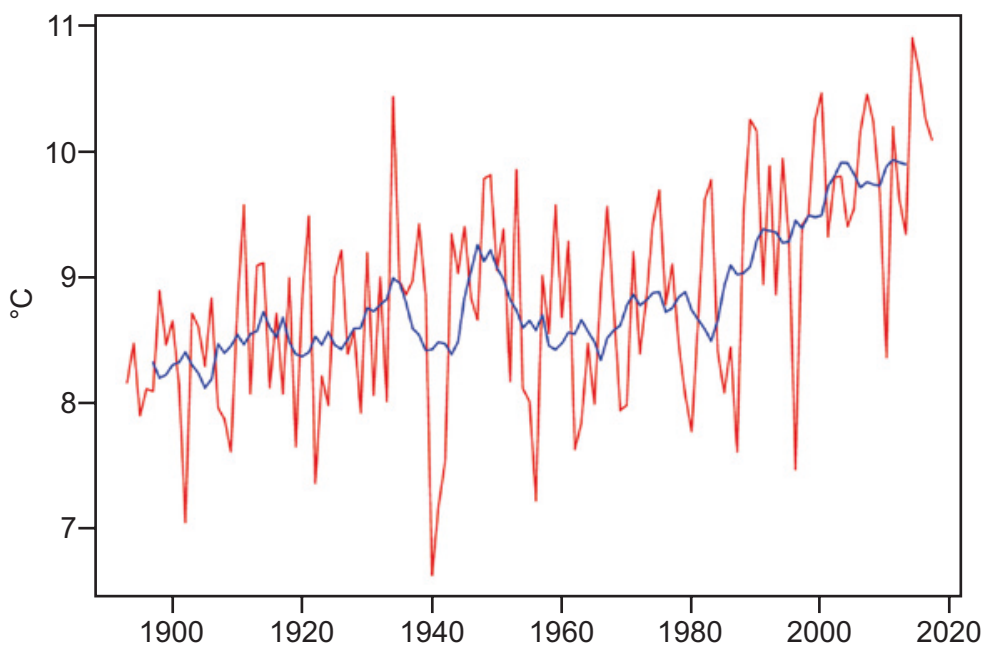

Entwicklung der Jahresmittel der Lufttemperatur an der Säkularstation Potsdam (rote Linie). Die blaue Linie ist eine zeitliche Glättung aus Neunjahresmittelwerten.

Datenquelle: Deutscher Wetterdienst

Abb.4.4 Entwicklung der mittleren Temperatur in Deutschland seit 1881. (Quelle: Deutscher Wetterdienst)

und räumliche Aggregation zu gewinnen. Dem Bedürfnis nach zeitlich und räumlich hochauflösenden Klimaprojektionen für die vorausschauende Identifizierung von Anpassungsstrategien steht diese Situation entgegen. Regionalstudien wie CC-LandStraD bewegen sich daher in einem besonderen Spannungsfeld. Einerseits leisten sie wichtige methodische Vorarbeiten für eine integrierte Betrachtung von Klimafolgen. Andererseits darf der exemplarische Charakter dieser Arbeiten nicht aus dem Auge verloren werden. Sie sind in der Lage, potenzielle Problemfelder, Anpassungsstrategien und Wirkungen von Anpassungsmaßnahmen zu quantifizieren. Aus der Beschäftigung mit diesen ergibt sich eine Bereicherung des Anpassungsportfolios, es kann hieraus jedoch keine normative Präferenz für bestimmte Anpassungsmaßnahmen gegenüber dem Klimawandel abgeleitet werden.

Das globale Emissionsgeschehen der letzten drei Jahrzehnte folgte in dieser Zeit dem sogenannten RCP-Szenario „RCP 8.5“. „RCP“ steht für „,representative concentration pathway“. Dabei handelt es sich um ein Szenario für die transiente Störung der Strahlungsbilanz an der oberen Grenzschicht der Atmosphäre bis zum Jahr 2100. Die Bezeichnung ,8.5“ entspricht dem zusätzlichen Strahlungsantrieb (RF, siehe oben) in $\mathrm{W} / \mathrm{m}^{2}$, um den die Strahlungsbilanz bis zum Ende des Jahrhunderts gestört wird (Burkett et al. 2014). Um diesen Wert würde sich unter sonst gleichen Bedingungen die langwellige atmosphärische Rückstrahlung erhöhen und damit die heutige Bilanz von einund ausstrahlender (kurz- und langwelliger) Strahlung verschieben.

In Abhängigkeit von der angenommenen Klimasensitivität $(\lambda)$ würde sich die globale Durchschnittstemperatur in einer Spanne zwischen 2,63 K $\left(\lambda=0,31 \mathrm{Km}^{2} / \mathrm{W}\right)$ und $4,81 \mathrm{~K}$ 
$\left(\lambda=0,56 \mathrm{Km}^{2} / \mathrm{W}\right.$ ) bis zum Jahr 2090 (Dekadenmittel 2081-2100) erhöhen (IPCC 2013). Es handelt sich dabei um die Spannweite zwischen dem 5. und 95. Perzentil aller Werte. Der Median aller berücksichtigten Modellsimulationen liegt bei $3,57 \mathrm{~K}\left(\lambda=0,42 \mathrm{Km}^{2} / \mathrm{W}\right)$. Bis zum Jahr 2060 entspricht dies einem mittleren Temperaturanstieg von 2,16 (1,55-2,99 K).

Der globale und regionale Temperaturanstieg des „RCP 8.5“-Szenarios bildete die Grundlage für das in CC-LandStraD in verschiedenen Klimafolgenbetrachtungen genutzte Klimaszenario. Es wurde mit dem Szenarienmodell STAR durch Filterung und Neukombination historischer Wetterreihen generiert (Gerstengarbe und Werner 2013; Gerstengarbe et al. 2013). Zielgröße der Filterung war die Erfüllung einer bestimmten Temperaturvorgabe. Die Temperaturvorgabe wurde für Deutschland aus den Ergebnissen von 21 globalen Klimamodellläufen zum Szenario „RCP 8.5“ ermittelt.

Die im Ergebnis entstandenen wärmeren Jahre waren im Sommer trockener und im Winter feuchter. Dies folgt aus dem Umstand, dass wärmere Sommer in Deutschland in der Regel eher auch trockener sind und wärmere Winter in der Regel auch feuchter. Ein Klimatrend wird somit aus der interannuellen Variabilität generiert. Ein solches Vorgehen lässt sich zwar pragmatisch rechtfertigen, eine eigene klimatische Plausibilität kann ihm jedoch nicht zugesprochen werden. Die Erwärmung im Szenario setzt eine jahreszeitliche Manipulation des Bewölkungsgrades, die sich aus den physikalischen Gründen des Klimawandels nicht ableiten lässt, voraus. Das Szenario musste daher extern plausibilisiert werden (Roers und Wechsung 2015; Wechsung und Wechsung 2015a, b).

Für die Sinnfälligkeit eines ,trockenere Sommer“-,,feuchtere Winter"-Szenarios sprachen verschiedene Gründe. Die saisonalen Änderungen entsprachen der generellen Erwartung, dass bei einer Erwärmung feuchte Verhältnisse feuchter und trocknere noch trockener würden (Collins et al. 2013). Dies entsprach auch den regionalen Trends: Der trockenere Osten Deutschlands verzeichnete bei den 30-Jahre-Trends zurückgehende Niederschläge, der feuchtere Westen zunehmende Niederschläge (eigene Ergebnisse). Regionale Auswertungen globaler Klimamodellrechnungen zeigten zudem, dass sich Deutschland im Übergangsbereich zwischen einer Region zunehmender Jahresniederschläge im Norden und einer Region abnehmender Niederschläge rund um das Mittelmeer befindet. Dieser Übergangsbereich verschiebt sich im Sommer nach Süden und im Winter nach Norden (Meehl et al. 2007). Die Szenarienauswahl fand nicht zuletzt unter dem Eindruck des Sommers 2003 statt, der als früher Bote des zu erwartenden Klimawandel eingestuft wurde.

In der Zwischenzeit liegen Ergebnisse sowohl von Modellrechnungen als auch statistischen Analysen vor, die daraufhin deuten, dass das in CC-LandStraD verwendete STAR-Szenario eher als eine trockene Variante des künftigen Klimas angesehen werden kann, die aber durchaus innerhalb des von den Modellen überstrichenen simulierten Bereiches liegt.

Um dies zu illustrieren, wird das STAR-Szenario hier nicht für sich stehend dargestellt, sondern mit Ergebnissen jüngster Klimamodellläufe verglichen (Tab. 4.2, 4.3, 4.4 und 4.5). Dabei handelt es sich einerseits um regional gemittelte Ergebnisse von ausgewählten globalen Klimamodellen und andererseits um Ergebnisse von regionalen Klimamodellen, die globale Simulationsrechnungen untersetzen. 
Tab.4.2 Saisonale, räumliche Mittelwerte (MW) und Standardabweichungen (SD) der Temperatur aus den drei Klimadatensätzen ISI-MIP, STARS und CORDEX für Deutschland zu den Perioden 1981-2010 und 2041-2070, sowie die Periodendifferenzen der Temperaturmittelwerte $\Delta \mathrm{T}$. (Quelle: T. Conradt)

\begin{tabular}{|c|c|c|c|c|c|}
\hline \multirow{2}{*}{$\begin{array}{l}\text { Jahr } \\
\text { Temperatur }\left[{ }^{\circ} \mathbf{C}\right]\end{array}$} & \multicolumn{2}{|l|}{ 1981-2010 } & \multicolumn{2}{|l|}{ 2041-2070 } & \multirow{2}{*}{$\begin{array}{l}\text { Änderung } \\
\Delta T\end{array}$} \\
\hline & MW & SD & MW & SD & \\
\hline \multicolumn{6}{|l|}{ Jahr } \\
\hline ISI-MIP & 8,99 & 0,69 & 11,65 & 1,11 & 2,66 \\
\hline STARS & 9,02 & 0,76 & 11,21 & 0,75 & 2,19 \\
\hline CORDEX & 8,85 & 0,72 & 10,77 & 1,02 & 1,92 \\
\hline \multicolumn{6}{|c|}{ Hydrologischer Sommer (Mai-Oktober) } \\
\hline ISI-MIP & 14,63 & 0,76 & 17,41 & 1,39 & 2,78 \\
\hline STARS & 14,61 & 0,65 & 16,59 & 0,56 & 1,98 \\
\hline CORDEX & 14,37 & 0,82 & 16,44 & 1,11 & 2,07 \\
\hline \multicolumn{6}{|c|}{ Hydrologischer Winter (April-November) } \\
\hline ISI-MIP & 3,29 & 1,14 & 5,82 & 1,18 & 2,53 \\
\hline STARS & 3,35 & 1,08 & 5,74 & 1,10 & 2,39 \\
\hline CORDEX & 3,23 & 1,15 & 5,00 & 1,42 & 1,77 \\
\hline
\end{tabular}

Tab. 4.3 Saisonale, räumliche Mittelwerte (MW) und Standartabweichungen (SD) der Globalstrahlung aus den drei Klimadatensätzen ISI-MIP, STARS und CORDEX für Deutschland zu den Perioden 1981-2010 und 2041-2070, sowie die Periodendifferenzen der Strahlungsmittelwerte $\Delta$ R. (Quelle: T. Conradt)

\begin{tabular}{|c|c|c|c|c|c|}
\hline \multirow{2}{*}{$\begin{array}{l}\text { Jahr } \\
\text { Globalstrahlung }\left[\mathrm{W} / \mathrm{m}^{2}\right]\end{array}$} & \multicolumn{2}{|l|}{ 1981-2010 } & \multicolumn{2}{|l|}{ 2041-2070 } & \multirow{2}{*}{$\begin{array}{l}\text { Änderung } \\
\Delta \mathbf{R}\end{array}$} \\
\hline & MW & SD & MW & SD & \\
\hline \multicolumn{6}{|l|}{ Jahr } \\
\hline ISI-MIP & 101 & 5,2 & 107,2 & 7,6 & 6,2 \\
\hline STARS & 117,6 & 4,9 & 126,3 & 4,7 & 8,7 \\
\hline CORDEX & 124,6 & 5,8 & 124,1 & 5,5 & $-0,5$ \\
\hline \multicolumn{6}{|l|}{ Hydrologischer Sommer } \\
\hline ISI-MIP & 145,4 & 8,4 & 156,1 & 11,8 & 10,7 \\
\hline STARS & 167,7 & 8,8 & 183,5 & 6,5 & 15,8 \\
\hline CORDEX & 177 & 8,9 & 178,3 & 9,7 & 1,3 \\
\hline \multicolumn{6}{|l|}{ Hydrologischer Winter } \\
\hline ISI-MIP & 56,2 & 3,9 & 57,8 & 4,6 & 1,6 \\
\hline STARS & 66,8 & 4,3 & 68,1 & 4,2 & 1,3 \\
\hline CORDEX & 72,2 & 4,9 & 69,9 & 4,5 & $-2,3$ \\
\hline
\end{tabular}


Tab.4.4 Saisonale, räumliche Mittelwerte (MW) und Standartabweichungen (SD) des Niederschlages aus den drei Klimadatensätzen ISI-MIP, STARS und CORDEX für Deutschland zu den Perioden 1981-2010 und 2041-2070 sowie die Periodendifferenzen der Niederschlagsmittelwerte $\Delta$ N. (Quelle: T. Conradt)

\begin{tabular}{|c|c|c|c|c|c|}
\hline \multirow{2}{*}{$\begin{array}{l}\text { Jahr } \\
\text { Niederschlag [mm] }\end{array}$} & \multicolumn{2}{|l|}{ 1981-2010 } & \multicolumn{2}{|l|}{ 2041-2070 } & \multirow{2}{*}{$\begin{array}{l}\text { Änderung } \\
\Delta \mathrm{N} \\
\end{array}$} \\
\hline & MW & SD & MW & SD & \\
\hline \multicolumn{6}{|l|}{ Jahr } \\
\hline ISI-MIP & 864 & 83 & 907 & 104 & 43 \\
\hline STARS & 800 & 99 & 740 & 84 & -60 \\
\hline CORDEX & 773 & 108 & 807 & 112 & 34 \\
\hline \multicolumn{6}{|c|}{ Hydrologischer Sommer } \\
\hline ISI-MIP & 453 & 59 & 460 & 82 & 7 \\
\hline STARS & 431 & 65 & 352 & 48 & -79 \\
\hline CORDEX & 418 & 67 & 414 & 77 & -4 \\
\hline \multicolumn{6}{|c|}{ Hydrologischer Winter } \\
\hline ISI-MIP & 414 & 54 & 450 & 60 & 36 \\
\hline STARS & 369 & 61 & 389 & 59 & 20 \\
\hline CORDEX & 357 & 72 & 392 & 76 & 35 \\
\hline
\end{tabular}

Tab. 4.5 Saisonale auf den Temperaturunterschied $(\Delta \mathrm{T})$ normierte Periodendifferenzen (20412070 minus 1981-2010) des Niederschlages $(\Delta N)$ und der Strahlung $(\Delta R)$ zu den Tab. 4.2 bis 4.4 für die drei Klimadatensätzen ISI-MIP, STARS und CORDEX. (Quelle: T. Conradt)

\begin{tabular}{|c|c|c|}
\hline & $\Delta \mathrm{N} / \Delta \mathrm{T}[\mathrm{mm} / \mathrm{C}]$ & $\Delta R / \Delta T\left[W / m^{2}{ }^{\circ} \mathrm{C}\right]$ \\
\hline \multicolumn{3}{|l|}{ Jahr } \\
\hline ISI-MIP & 16,17 & 2,33 \\
\hline STARS & $-27,40$ & 3,97 \\
\hline CORDEX & 17,71 & $-0,26$ \\
\hline \multicolumn{3}{|c|}{ Hydrologischer Sommer } \\
\hline ISI-MIP & 2,52 & 3,85 \\
\hline STARS & $-39,90$ & 7,98 \\
\hline CORDEX & $-1,93$ & 0,63 \\
\hline \multicolumn{3}{|c|}{ Hydrologischer Winter } \\
\hline ISI-MIP & 14,23 & 0,63 \\
\hline STARS & 8,37 & 0,54 \\
\hline CORDEX & 19,77 & $-1,30$ \\
\hline
\end{tabular}


Die regional gemittelten globalen Klimamodellsimulationen entstammen der ISIMIP-Modellstudie, in der fehlerkorrigierte Werte (WATCH Forcing Data; Weedon et al. 2011) folgender Globaler Klimamodelle verwendet wurden: HadGEM2-ES (Met Office Hadley Centre), IPSL-CM5A-LR (Institut Pierre-Simon Laplace), MIROC-ESM-CHEM (Japan Agency for Marine-Earth Science and Technology), GFDL-ESM2M (NOAA Geophysical Fluid Dynamics Laboratory), NorESM1-M (Norwegian Climate Centre). Als Fehler wird (analog zu Wettervorhersagen) die Abweichung zwischen Simulationen für die Vergangenheit und Beobachtungswerten aus demselben Zeitraum gewertet (Hempel et al. 2013). Es wird davon ausgegangen, dass dieser Fehler auch in der Zukunft auftreten wird und damit ein Verfahren der Fehlerkorrektur, das für die Vergangenheit entwickelt wurde, auch auf Zukunftssimulationen übertragen werden kann, wobei die langfristigen Änderungstrends hiervon unbeeinflusst sein sollten.

Die regionalisierten Klimamodellläufe sind das Ergebnis einer Kombination von globalen und regionalen Klimamodellen der CORDEX Europa Initiative (Jacob et al. 2013). Als Antrieb wurde das Szenario „RCP 8.5“ verwendet. Die Modellkombination umfassten: ICHEC-EC-EARTH/KNMI-RACMO22E, ICHEC-EC-EARTH/SMHI-RCA4, MOHC-HadGEM2-ES/SMHI-RCA4, MPI-M-ESM-LR/MPI-CSC-REMO2009. Die Modelldatensätze wurden mit einem Verfahren zur Fehlerkorrektur nach Gobiet et al. (2015) korrigiert. Als Datensatz für die Fehlerkorrektur diente: Europäischer Datensatz E-OBS (Haylock et al. 2008). Die räumlich grob aufgelösten Ergebnisse der globalen Klimamodelle liefern den Antrieb für die feiner aufgelösten regionalen Klimamodelle. Rückwirkungen werden dabei vernachlässigt. Die regionalen Modelläufe werden analog zum ISI-MIP-Projekt jedoch unter Nutzung eines anderen Verfahrens fehlerkorrigiert.

Eine zusammenfassende Darstellung der für Deutschland durch die verschiedenen Modelle simulierten Szenarienklimatologie wird im Folgenden für die simulierten Reihen für Temperatur, Niederschlag und Globalstrahlung vergleichend dargestellt und erläutert. Generell sollten die zeitlichen Schwankungsbereiche der Datenserien sich für die Zeit vor 2010 stark überlappen. Die fehlerkorrigierten Reihen (ISI-MIP, CORDEX Europa) können in dieser Zeit nur dann deutlich voneinander abweichen, wenn es Unterschiede zwischen den Datensätzen gibt, die der Fehlerkorrektur zugrunde lagen. Bei der Temperatur und dem Niederschlag ist dies nicht der Fall.

Die Reihen der Globalstrahlung hingegen zeigen einen solchen Unterschied. Die ISIMIP-Reihen zeigen eine deutliche Unterschätzung des beobachteten Strahlungsniveaus, die sich dann auch folgerichtig in die Zukunft fortsetzt. Diese Unterschätzung ist dem verwendeten globalen Datensatz geschuldet, in dessen Generierung offensichtlich weniger regionale Beobachtungsdaten eingingen als aus regionaler Sicht notwendig. Diese Diskrepanz unterstreicht die Notwendigkeit, regional verfügbare Daten auch umfassend global verfügbar zu machen, da ansonsten in global ausgerichteten Klimastudien die darin enthaltenen regionalen Betrachtungen unnötig mit Fehlern belastet werden.

Die Reproduktion der rezenten Verhältnisse von Temperatur und Niederschlag durch die drei Datensätze ist jedoch hinreichend, um die Projektionen bezüglich dieser beiden 
Variablen zu vergleichen. Zwischen den Temperaturprojektionen gibt es kaum relevante Unterschiede (vgl. Tab. 4.2). Dies ist zu erwarten, da sie erstens dem gleichen Strahlungsantrieb folgen (vgl. Tab. 4.3) und zweitens die regionalen Realisierungen der globalen Erwärmung weitaus weniger streuen als jene der Niederschläge.

Bei den Niederschlägen zeigt sich eine untere Randlage des STAR-Szenarios im Schwankungsband, die besonders stark im hydrologischen Sommer (Mai-Oktober) ausgeprägt ist und sich dann bis auf die Jahresniederschlagssummen durchprägt (vgl. Tab. 4.4). Dieser Befund wird durch die in Tab. 4.4 dargestellten Periodendifferenzen bestätigt. Das STAR-Szenario unterstellt für CC-LandStraD den stärksten Rückgang der Jahres- und Sommerniederschläge. Dies wird noch deutlicher, wenn man die Temperaturnormierten Änderungen vergleicht (vgl. Tab. 4.5).

Wenn man dem hier genutzten Ensemble von Klimamodellrechnungen Repräsentanz zubilligt und gleichzeitig berücksichtigt, dass die STAR-Szenarien eine externe Legitimierung benötigen, lässt sich schlussfolgern, dass das STAR-Szenario eine eher trockene Version des künftigen Klima beschreibt. Damit ist jedoch gleichzeitig gesagt, dass sich das STAR-Szenario durchaus im Schwankungsbereich der aus heutiger Sicht möglichen Klimata befindet. Die hier vorgelegten Klimafolgenbetrachtungen sind also insbesondere geeignet, die Vulnerabilität der Landnutzung gegenüber einem wärmeren und trockeneren Klima bzw. gegenüber den Trockenphasen innerhalb eines wärmeren und leicht feuchter werdenden Klima darzustellen. Diese Einordnung kann sich künftig durchaus ändern. Eine fortlaufende Hinterfragung dieser Einordnung durch spätere Analysen und Modellrechnungen ist deshalb ausdrücklich zu begrüßen.

Mit Blick auf die bis zum Ende des Jahrhunderts erwarteten Änderungen des Klimas wurden zwei Klimaszenarien durch das regionale statistische Klimamodell STAR berechnet, welches mittlere Temperaturerhöhungen für die Zukunft (hier 2007-2060 bzw. 2100) im Vergleich zu einem Referenzzeitraum (hier 1980-2006) in regionale differenzierte Klimatrends übersetzt. Um eine breite Spanne möglicher klimatischer Veränderungen abzubilden, wurde für die Klima-Referenzprojektion „,OK“ keine Temperaturerhöhung im jährlichen Mittel für Deutschland gegenüber dem Referenzzeitraum unterstellt. Das „RCP 8.5“-ECHAM6-Szenario für Deutschland beruht auf einem mittleren Klimatrend für Deutschland, der sich aus der Simulation des globalen Klimazirkulationsmodel ECHAM6 (Stevens et al. 2013), angerieben durch das Emissionsszenario „RCP 8.5“, für 2007-2100 ergibt (siehe oben). Der „,mittlere Temperaturtrend“ ergibt sich aus dem Vergleich der Ergebnisse für Deutschland verschiedener globaler Klimazirkulationsmodelle, die jeweils mit dem „RCP 8.5“ angetrieben wurden. Die beiden Klimaszenarien sind in Abb. 4.5 dargestellt.

Die Auswirkungen der erwarteten Klimaveränderungen auf die Ergebnisse der Strategien werden mittels qualitativer und (wenn möglich) quantitativer Abschätzungen beschrieben. 


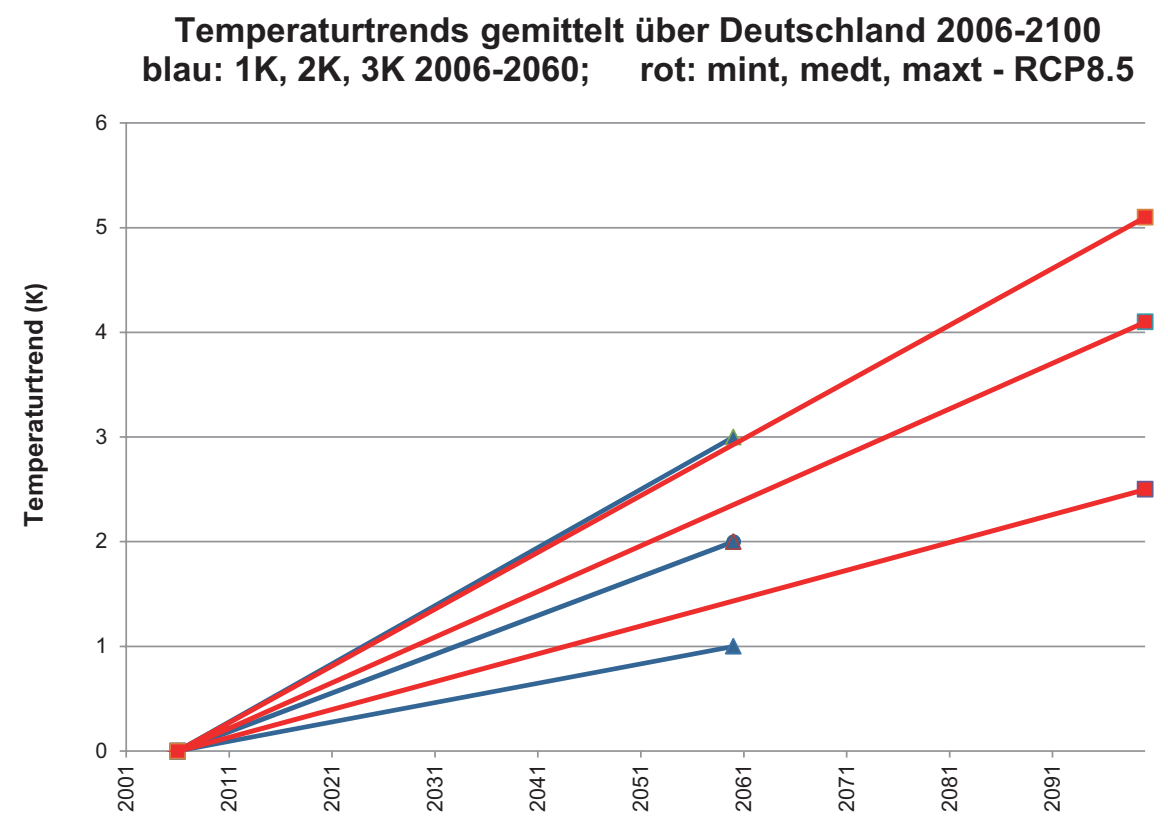

Abb. 4.5 Darstellung der Temperaturtrends für Deutschland, welche in CC-LandStraD verwendet werden. $\mathrm{Blau}=0 \mathrm{~K}$, rot $=\mathrm{RCP} 8.5$-ECHAM6

\subsection{Ergebnisse der Siedlungs- und Verkehrsflächenmodellierung}

Roland Goetzke und Jana Hoymann

\section{Zusammenfassung}

Durch politisch-strategische oder planerische Maßnahmen lässt sich die Siedlungsflächenentwicklung sowohl in ihrer Größenordnung als auch in ihrer räumlichen Ausprägung steuern. Je nachdem, welches übergeordnete Ziel verfolgt wird (Klimaschutz, Klimaanpassung, Natur- und Umweltschutz) können sehr unterschiedliche Maßnahmen zum Tragen kommen, die sich teilweise ergänzen oder auch gegensätzlich wirken können. In diesem Kapitel wird beschrieben, wie Maßnahmen im Siedlungs- und Verkehrsbereich im Modell abgebildet werden (Annahmen und Spezifikationen) und wie sie quantitativ und qualitativ die Landnutzungsentwicklung beeinflussen (Auswirkungen der Maßnahme). Eine Referenzprojektion, die die Siedlungsflächenentwicklung bis 2030 unter der Annahme aufzeigt, dass keine neuen Maßnahmen ergriffen werden, bildet die Basis. Anschließend werden zehn einzelne Maßnahmen und deren Modellergebnisse beschrieben. Schließlich wird die Wirkung von Maßnahmenbündeln innerhalb von übergeordneten Landnutzungsstrategien aufgezeigt. Die Maßnahmenwirkung wird mit Hilfe von Indikatoren bewertet. 


\subsubsection{Referenzprojektion Flächennutzung}

Die Bevölkerungsprognose 2030 des BBSR aus dem Jahr 2012, die den hier durchgeführten Berechnungen zugrunde liegt, geht davon aus, dass die aktuelle Bevölkerungszahl bis zum Jahr 2030 auf 78,6 Mio. Einwohner sinken wird. Der Bevölkerungsrückgang ist auf die bereits seit Jahrzehnten zu beobachtenden niedrigen Geburtenraten und die wieder steigenden Sterberaten zurückzuführen. Die in dieser Prognose angenommenen Wanderungsgewinne steigen von 50.000 im Jahr 2009 auf 150.000 im Jahr 2014, um bis zum Ende des Projektionszeitraums nahezu konstant zu bleiben. In der hier verwendeten Prognose können die Wanderungsgewinne den natürlichen Bevölkerungsrückgang langfristig nicht mehr ausgleichen. Wanderungsgewinne verzeichnen vor allem die wirtschaftlich starken Großstädte.

Obwohl die Bevölkerungszahl bereits seit einigen Jahren zurückgeht, nimmt die Anzahl der Haushalte bisher kontinuierlich zu, wobei die Zunahmen in den aktuellen Trends langsamer stattfinden. Im Jahr 2010 existierten in Deutschland 40,3 Mio. Haushalte. Bis zum Jahr 2025 steigt die Anzahl laut der Raumordnungsprognose auf 41,2 Mio. an, um anschließend bis 2030 wieder auf 41,0 Mio. zu sinken. Das Haushaltsbildungsverhalten mit der Tendenz zu kleineren Haushalten ist also künftig nicht mehr in der Lage, die demographische Entwicklung auszugleichen.

Das BBSR hat im Jahr 2015 die aktuelle Raumordnungsprognose 2035 veröffentlicht. In dieser wird den aktuell hohen Wanderungsgewinnen (ohne Zuwanderung von Geflüchteten) Rechnung getragen. Danach liegt der Außenwanderungssaldo aktuell und in den kommenden Jahren bei 400.000. Bis 2035 reduziert sich der Wanderungsgewinn dann wieder auf das langfristige Mittel von 200.000. Die hohen Wanderungsgewinne kompensieren derzeit den natürlichen Bevölkerungsrückgang. Langfristig ist dies allerdings nicht möglich. Im Jahr 2035 werden 78,2 Mio. Einwohner erwartet (BBSR 2015a, b). Diese aktuelle Prognose konnte aufgrund des Projektfortschritts in den Ergebnissen dieses Projektes nicht mehr verwendet werden. Es ist aber davon auszugehen, dass sich die hier dargestellten Entwicklungstrends, v. a. hinsichtlich der Flächennutzung, lediglich zeitlich verschieben und nicht grundsätzlich ändern. Das heißt, dass vorübergehend mit einem höheren Flächenzuwachs gerechnet werden kann, der dann in Zukunft aber wieder deutlich zurïckgeht.

Die tägliche Neuinanspruchnahme von Flächen für Siedlung und Verkehr wird von 73 ha/Tag im Zeitraum 2010 bis 2013 auf ca. 45 ha/Tag im Zeitraum 2026 bis 2030 zurückgehen. Das 30-Ha-Ziel der Nachhaltigkeitsstrategie wird demnach trotz eines deutlich rückläufigen Trends nicht erreicht. Davon werden allerdings nur rund 19,5 ha für Gebäude- und Freiflächen beansprucht. Für Erholungs- und Grünflächen beträgt die 
tägliche Neuinanspruchnahme 2030 noch knapp 9 ha und für Verkehrsflächen 15,5 ha. Die tägliche Neuinanspruchnahme für Betriebsflächen (ohne Abbauland) beträgt dann nur noch 1 ha. Ohne die Erholungs- und Grünflächen liegt die Flächenneuinanspruchnahme 2030 bei knapp unter 36 ha/Tag. Auch die räumlichen Unterschiede in der Flächenentwicklung, die bereits in der Vergangenheit beobachtet wurden, setzen sich fort. So wächst die künftige Siedlungs- und Verkehrsfläche vor allem im verdichteten Umland westdeutscher Großstädte und um Berlin. In einigen Regionen Süddeutschlands steigt die Flächenneuinanspruchnahme zunächst sogar noch.

Abb. 4.6 gibt einen auf Gemeindeebene aggregierten Überblick über die Siedlungsund Verkehrsflächenentwicklung in Deutschland bis 2030. Die Regionen mit künftig hoher Flächeninanspruchnahme sind vor allem die Regionen, die bereits heute einen hohen Anteil an Siedlungs- und Verkehrsfläche aufweisen. Im Besonderen sind das die Regionen um die großen Metropolen Hamburg, Berlin, München und Rhein-Main. Gleichzeitig sind die Regionen mit besonders geringer Flächeninanspruchnahme deutlich erkennbar. Vor allem in Sachsen-Anhalt und Mecklenburg-Vorpommern sowie dem Ruhrgebiet, dem Schwarzwald und der Schwäbischen Alb sind aufgrund der demographischen Entwicklung nur noch geringe Zuwächse der Siedlungs- und Verkehrsfläche zu erwarten.

Die verbleibende Produktionsfläche für die Landwirtschaft sinkt in der Referenzprojektion, bedingt durch den Zuwachs an Siedlungs- und Verkehrsflächen sowie Waldflächen, um knapp $2 \%$ von $186.193 \mathrm{~km}^{2}$ im Jahr 2013 auf $180.188 \mathrm{~km}^{2}$ im Jahr 2030 (Abb. 4.7). Dabei finden die größten prozentualen Rückgänge in den kreisfreien Großstädten und Ballungsräumen statt, in denen die Landwirtschaftsfläche ohnehin nur einen geringen Anteil an der Bodenfläche hat (Abb. 4.7 und 4.8). Die größten absoluten Rückgänge der Landwirtschaftsfläche sind in großen, stark landwirtschaftlich geprägten Kreisen mit hoher Siedlungsdynamik zu verzeichnen, wie im Ems- oder Münsterland oder der Region Hannover. Die Waldfläche nimmt prozentual vor allem in waldarmen Regionen zu (Abb.4.9). Absolut sind die Zunahmen auf Grenzertragsstandorten am höchsten.

\subsubsection{Analyse ausgewählter Maßnahmen „Siedlung und Verkehr}

Zu den Maßnahmen, die vor allem den Umfang der Siedlungs- und Verkehrsflächenentwicklung beeinflussen, zählen die Stärkung der Innenentwicklung, die Ausschöpfung der baulichen Dichte im Neubau, die Reduzierung der Flächeninanspruchnahme durch Verkehr und der Rückzug aus der Fläche. Die für die Modellierung getroffenen Spezifikationen und Annahmen zur Ausgestaltung dieser Maßnahmen sowie deren Auswirkungen auf die Flächennutzung sind im Folgenden zusammenfassend in Tab. 4.6 dargestellt. 
a

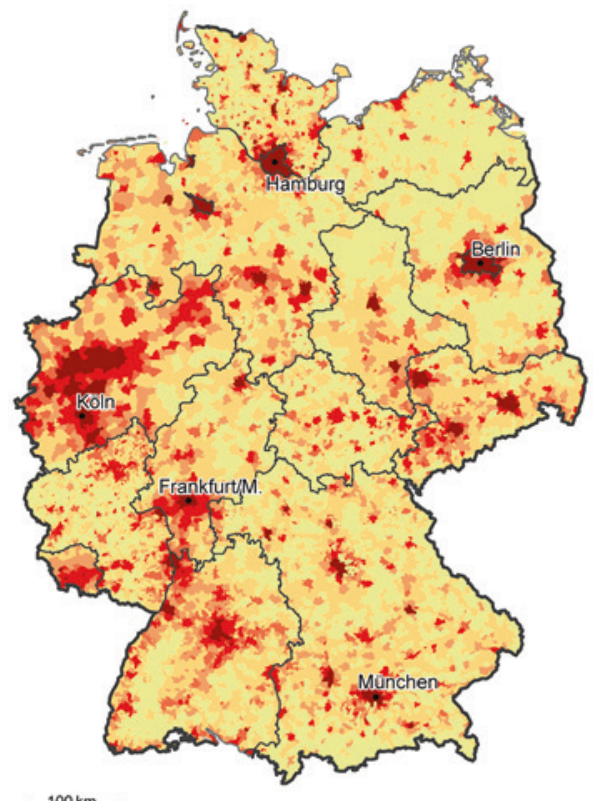

Anteil der Siedlungs- und Verkehrsfläche an der Katasterfläche 2010 in \% b

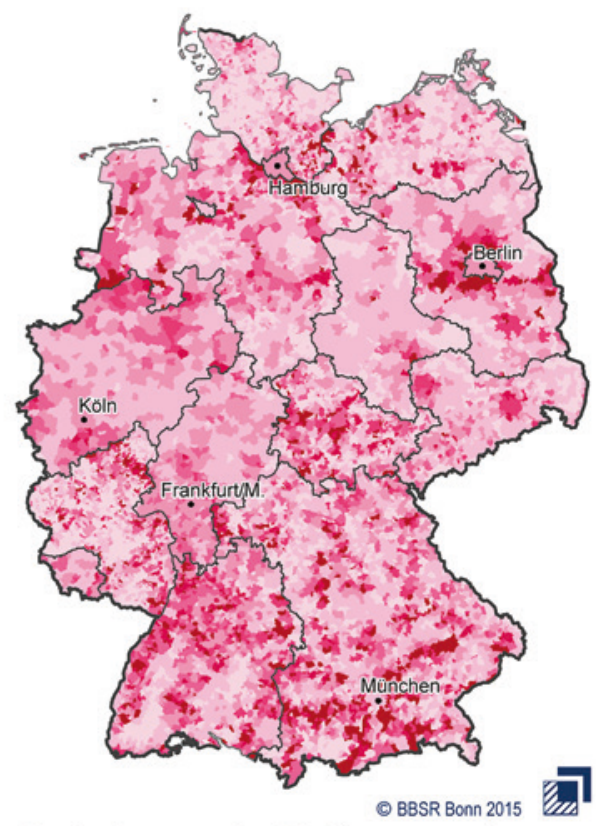

Veränderung der Siedlungs- und Verkehrsfläche zwischen 2010 und 2030 in \%

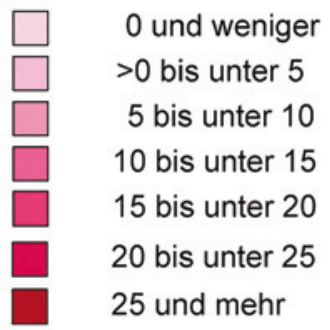

Quelle: Laufende Raumbeobachtung des BBSR, GWS Osnabrück 2012 ,

Berechnungen des BBSR Geometrische Basis: BKG/BBSR, Gemeinden 31.12.2012

Bearbeitung: J. Hoymann / R. Goetzke

Abb. 4.6 Entwicklung der Siedlungs- und Verkehrsfläche auf Gemeindesebene, Anteil der Siedlungs- und Verkehrsfläche 2010 (a) und Veränderung der Siedlungs- und Verkehrsfläche zwischen 2010 und 2030 (b)

\subsubsection{Stärkung der Innenentwicklung}

\section{Annahmen und Spezifikation der Maßnahme}

Es wird die Annahme getroffen, dass Flächenrecycling in stärkerem Umfang als bisher durchgeführt wird. Das bedeutet, dass vorhandene Brachflächen und Baulücken leichter 


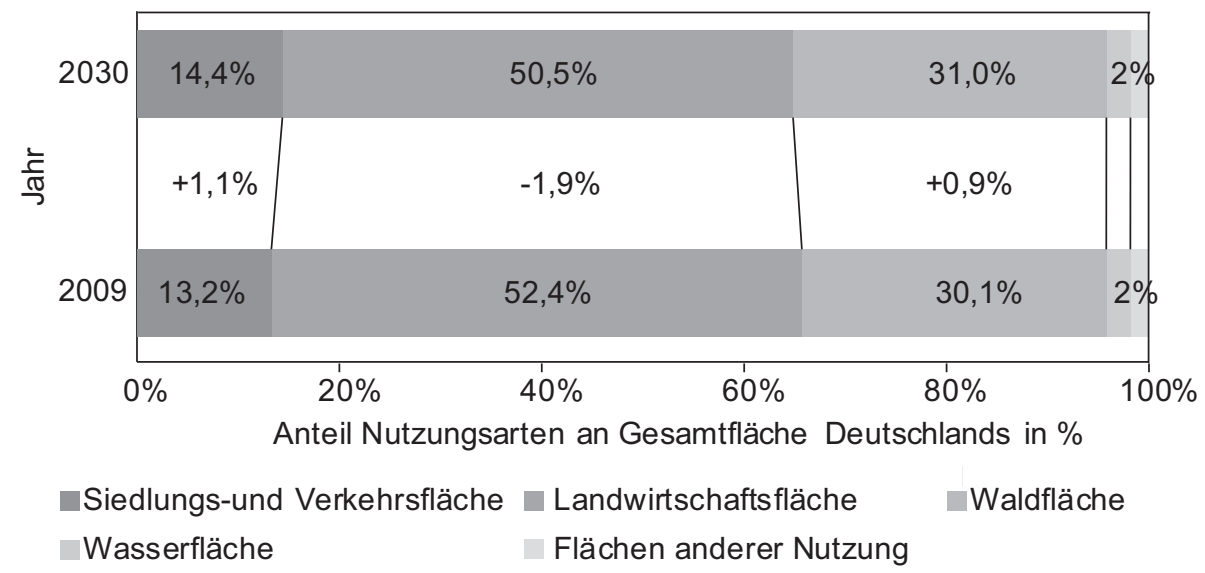

Abb. 4.7 Bodenfläche in Deutschland nach Nutzungsarten 2009 und 2030. (Datenquelle: Statistisches Bundesamt 2015; GWS Osnabrück 2012)

mobilisiert werden können. Allein durch die Nutzung von Brachflächen und Baulücken im Innenbereich könnten laut einer aktuellen Studie insgesamt 120.000 bis 165.000 ha Flächenpotenziale genutzt werden (BBSR 2013). Dies entspricht mehr als einem Drittel der bis 2030 geschätzten Nachfrage. Da etwa $70 \%$ hiervon aber nur schwer oder gar nicht aktivierbar sind und Angebot und Nachfrage außerdem nicht immer zusammenkommen, liegt die Wirksamkeit der Maßnahme deutlich unter den potenziell verfügbaren Brachflächen und Baulücken. Neben der vorrangigen Nutzung dieser Flächen beinhaltet die Maßnahme auch die Nutzung von Gebäudeleerständen und die Nutzung von Nachverdichtungsmöglichkeiten. Diese Handlungsoptionen sind in $\S 1$ a Abs. 2 BauGB seit der Novelle 2013 aufgeführt und werden in dem hier modellierten Maßnahmenbündel konsequent angewendet.

Die Nutzung von Wohnungsleerständen verringert zudem die Siedlungsflächennachfrage. Darüber hinaus wird im Bestand nachverdichtet. Dabei wird je nach derzeit vorhandener baulicher Dichte diese moderat angehoben. Auch dies reduziert die Siedlungsflächennachfrage.

\section{Auswirkungen der Maßnahme}

Unter Annahme einer konsequenten Mobilisierung von Brachflächen und Baulücken, der gezielten Wiedernutzung von Leerständen und einer moderaten Anhebung der Nachverdichtung im Bestand könnte die tägliche Flächenneuinanspruchnahme durch Siedlungs- und Verkehrsflächen im Zeitraum 2026 bis 2030 von 45 ha in der Referenzprojektion auf etwas mehr als 32 ha pro Tag zurückgehen. Das Innenentwicklungspotenzial würde somit theoretisch den Flächenbedarf für mehr als zehn Jahre decken 


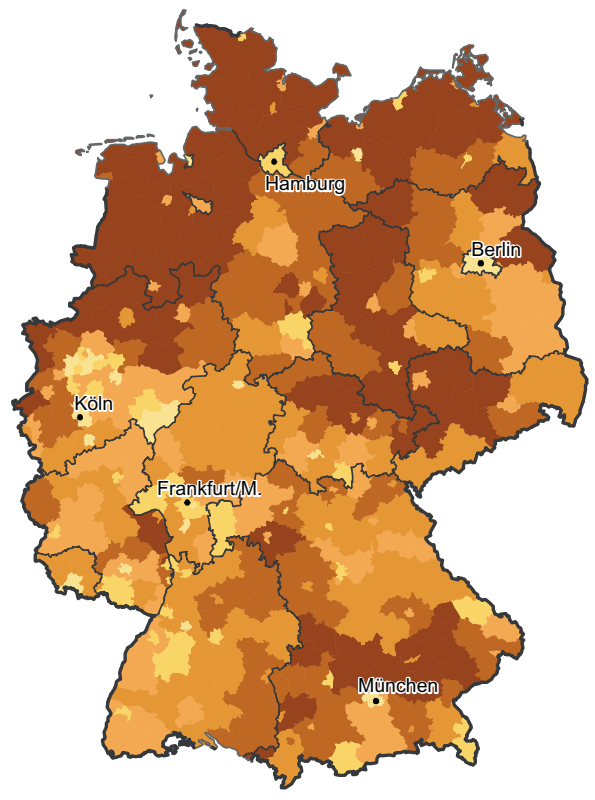

$100 \mathrm{~km}$

Anteil der Landwirtschaftsfläche an der Katasterfläche 2009 in Prozent

\begin{tabular}{|c|c|}
\hline & bis unter \\
\hline 20 & bis unter \\
\hline 30 & bis unter \\
\hline 40 & bis unter \\
\hline 50 & bis unter \\
\hline 60 & und mehr \\
\hline
\end{tabular}

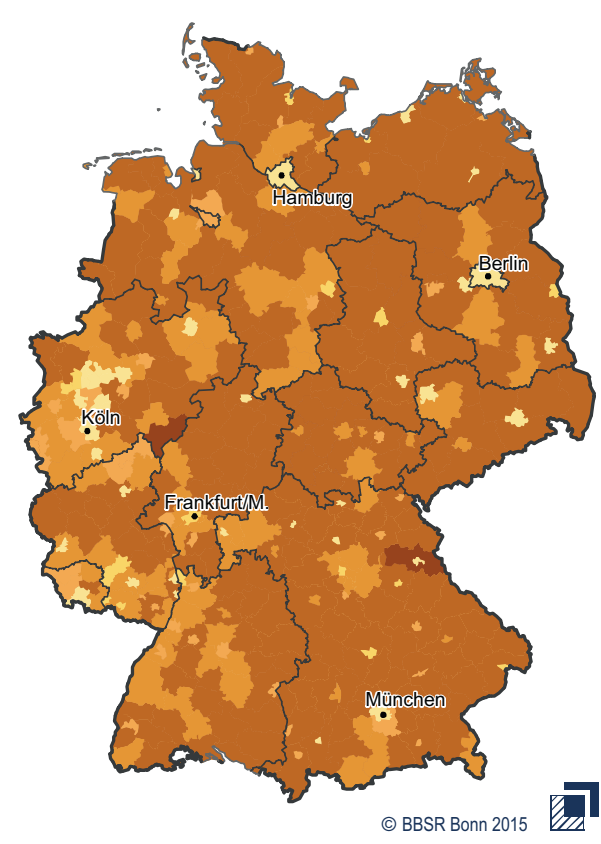

Veränderung der Landwirtschaftsfläche 2009 bis 2030 in Prozent

\begin{tabular}{lllr}
\hline & & bis unter & -20 \\
$\square \quad-20$ & bis unter & -15 \\
$\square$ & -15 bis unter & -10 \\
$\square \quad-10 \quad$ bis unter & -5 \\
$\square \quad-5 \quad$ bis unter & 0 \\
$\square$ & Zuwachs
\end{tabular}

Datenbasis: Laufende Raumbeobachtung des BBSR, GWS Osnabrück 2012, Berechnungen des BBSR

Geometrische Datenbasis: BKG/BBSR Kreise, 31.12.2012

Bearbeitung: R. Goetzke

Abb. 4.8 Landwirtschaftsfläche in den Kreisen Deutschlands 2009 und Entwicklung bis 2030

können. Durch konsequente Innenentwicklung ließe sich das 30-ha-Ziel der Nachhaltigkeitsstrategie annähernd erreichen. Nach den Modellergebnissen würden im Jahr 2030 im Vergleich zur Referenzprojektion rund 130.000 ha Landwirtschaftsfläche weniger für Siedlungs- und Verkehrszwecke in Anspruch genommen werden (vgl. Tab. 4.6).

Der Rückgang fällt nicht noch höher aus, da die Nachfrage nach Wohnraum und die Verfügbarkeit von Gebäudeleerständen oder Brachflächenbeständen oft in unterschied- 

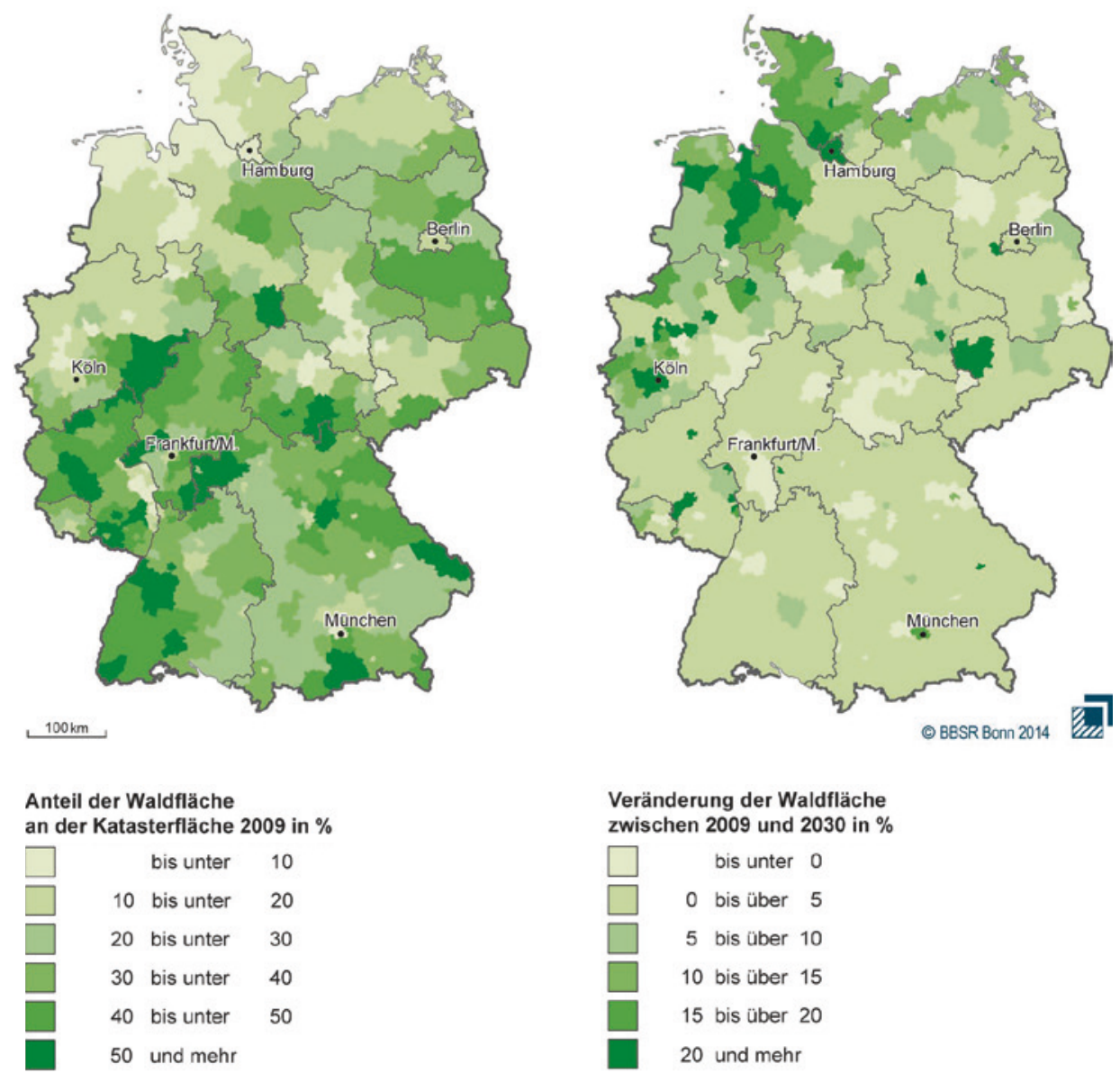

Datenbasis: Laufende Raumbeobachtung des BBSR, DESTATIS, GWS Osnabrück 2012,

Geometrische Datenbasis: BKG/BBSR Kreise, 31.12.2012 Bearbeitung: J. Hoymann

Abb. 4.9 Waldfläche in den Kreisen Deutschlands 2009 und Entwicklung bis 2030

lichen Regionen liegen. Bei Aufschlüsselung des Indikators „Zunahme der Siedlungsund Verkehrsfläche“ in die einzelnen Nutzungsarten der Siedlungs- und Verkehrsfläche fällt auf, dass der Rückgang der Neuinanspruchnahme vor allem von der Gebäude- und Freifläche getragen wird (vgl. Abb. 4.10). Der leichte Rückgang der Verkehrsflächenentwicklung ist eng mit der Gebäude- und Freiflächenentwicklung verknüpft, da durch die geringere Ausweitung der Siedlungsflächen weniger Erschließungsstraßen benötigt werden. 


\begin{tabular}{|c|c|c|c|c|c|c|c|}
\hline & $\begin{array}{l}\dot{\hat{\beta}} \\
\dot{0} \\
\dot{0}\end{array}$ & $\begin{array}{l}9 \\
0 \\
0\end{array}$ & $\begin{array}{c}0 \\
0 \\
0 \\
i \\
\end{array}$ & $\begin{array}{c}0 \\
i^{2} \\
n^{2}\end{array}$ & $\begin{array}{l}0 \\
i \\
\end{array}$ & 8 & $\stackrel{8}{1}$ \\
\hline & 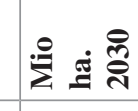 & $\frac{n}{n^{n}}$ & $\begin{array}{l}\text { di } \\
\text { i }\end{array}$ & $\begin{array}{l}\pi \\
\text { ת. } \\
0\end{array}$ & $\vec{\sigma}$ & $\stackrel{0}{0}$ & $\begin{array}{ll}8 & 8 \\
\underbrace{0} & \stackrel{ \pm}{=}\end{array}$ \\
\hline \multirow{2}{*}{ 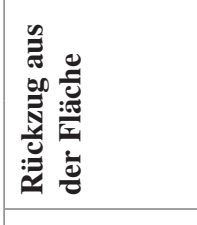 } & $\begin{array}{l}\dot{\hat{\beta}} \\
\dot{0} \\
\dot{0}\end{array}$ & $\begin{array}{l}0 \\
n \\
0 \\
1\end{array}$ & $\begin{array}{l}8 \\
0 \\
1 \\
1\end{array}$ & $\begin{array}{l}q \\
0 \\
i\end{array}$ & $\begin{array}{l}0 \\
i \\
\end{array}$ & 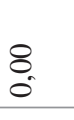 & $\frac{0}{0}:$ \\
\hline & 产产 & $\vec{z}$ & $\overrightarrow{\vec{j}}$ & $\begin{array}{l}0 \\
n \\
0 \\
0\end{array}$ & $\tilde{\sigma}$ & $\frac{0}{0}$ & 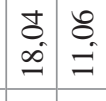 \\
\hline \multirow{2}{*}{ 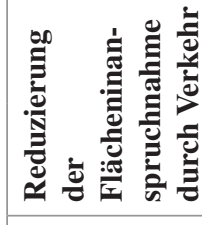 } & $\dot{\vec{\theta}} \dot{\overrightarrow{\tilde{E}}}$ & $\begin{array}{l}0 \\
0 \\
0 \\
1 \\
1\end{array}$ & 8 & $\begin{array}{c}0 \\
\text { c. } \\
0 \\
i\end{array}$ & $\stackrel{?}{i}$ & 8 & 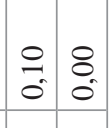 \\
\hline & ¿ & $=$ & $\begin{array}{l}\mathscr{B} \\
\text { i }\end{array}$ & : & 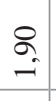 & $\stackrel{2}{0}$ & 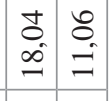 \\
\hline \multirow{2}{*}{ 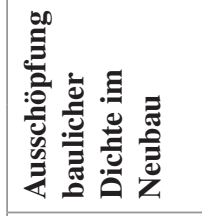 } & $\dot{\overrightarrow{3}}$ & $\begin{array}{l}8 \\
8 \\
0 \\
1\end{array}$ & $\underset{i}{i}$ & $\begin{array}{l}\text { q. } \\
\text { p. } \\
i\end{array}$ & $\begin{array}{l}q \\
q \\
i\end{array}$ & : & वृ : \\
\hline & 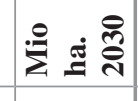 & o & $\begin{array}{l}8 \\
i\end{array}$ & 它 & $\vec{S}$ & $\stackrel{0}{0}$ & $\begin{array}{l}8 \\
\stackrel{8}{0} \\
\infty \\
\stackrel{0}{=} \\
=\end{array}$ \\
\hline \multirow{2}{*}{ 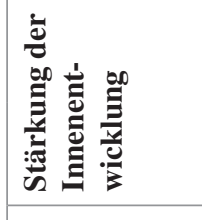 } & $\dot{\hat{\phi}} \dot{0}$ & $\begin{array}{l}0 \\
\text { n. } \\
i \\
1\end{array}$ & $\stackrel{0}{f}$ & 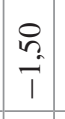 & $\begin{array}{c}0 \\
0 \\
0 \\
1\end{array}$ & 8 & $\begin{array}{l}R \\
0 \\
0\end{array}$ \\
\hline & 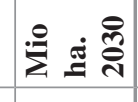 & 官 & $\begin{array}{l}\text { त̂ } \\
\text { in }\end{array}$ & b & $\stackrel{.}{-}$ & $\frac{0}{0}$ & 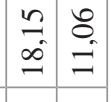 \\
\hline \multirow{3}{*}{ 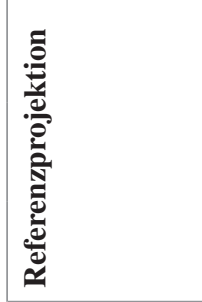 } & 을 & $\begin{array}{l}0 \\
\substack{1 \\
\infty \\
\infty}\end{array}$ & 品 & $\begin{array}{l}R \\
0 \\
0\end{array}$ & & ते & $\begin{array}{cc}\infty & 0 \\
0 & 0 \\
0 & 0 \\
4\end{array}$ \\
\hline & 言离产 & $\frac{m}{i n}$ & $\begin{array}{l}\mathscr{B} \\
\text { i }\end{array}$ & . & $\vec{\Xi}$ & $\stackrel{0}{0}$ & $\begin{array}{ll}8 \\
0 \\
0\end{array}$ \\
\hline & 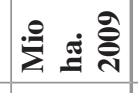 & $\begin{array}{l}\text { 亲 } \\
\dot{q}\end{array}$ & 年 & $\begin{array}{l}0 \\
g \\
0\end{array}$ & & $\stackrel{\infty}{0}$ & 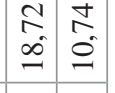 \\
\hline & & 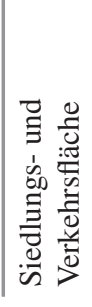 & 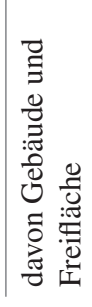 & 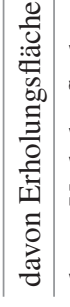 & & 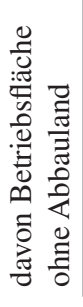 & 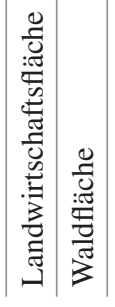 \\
\hline
\end{tabular}




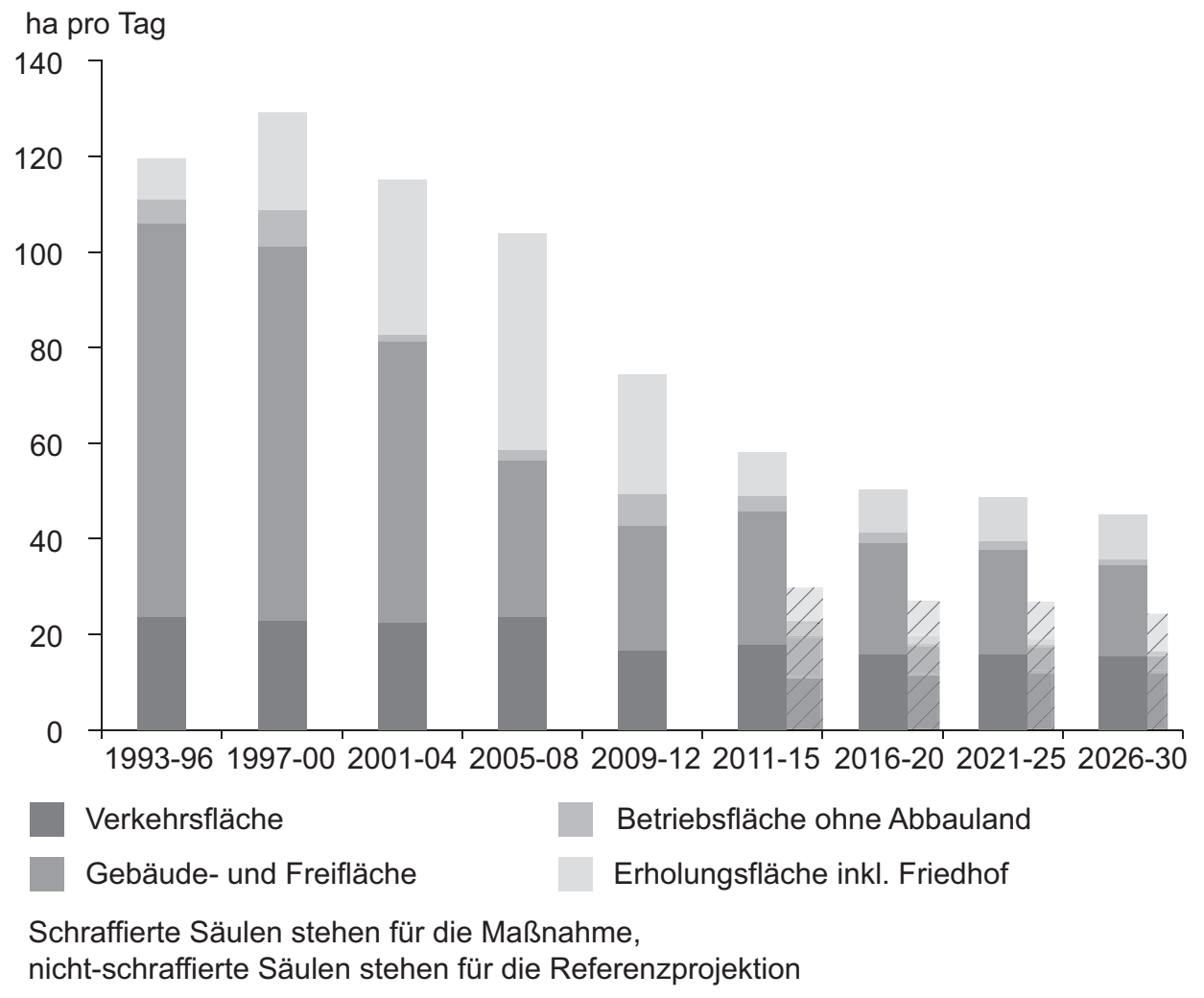

Abb. 4.10 Veränderung der der Siedlungs- und Verkehrsfläche 1993 bis 2030 für die Szenarien „Referenzprojektion“ und „Stärkung der Innenentwicklung“ (schraffierte Säulen stehen für die Maßnahme, nicht-schraffierte Säulen stehen für die Referenzprojektion)

Räumlich betrachtet wirkt die Maßnahme „Stärkung der Innenentwicklung“ sehr unterschiedlich (vgl. Abb. 4.11). In einigen Regionen könnte durch stärkere Innenentwicklung mehr als die Hälfte der Siedlungsflächennachfrage gedeckt werden. Beispiele hierfür sind Berlin, Teile des Ruhrgebiets oder das Rhein-Main-Gebiet östlich von Frankfurt a. M. In anderen von starkem Siedlungsflächenzuwachs geprägten Regionen kann die Innenentwicklung jedoch nur einen Teil der Flächennachfrage decken. Dies hängt einerseits damit zusammen, dass bei insgesamt hoher Nachfrage die Potenziale bereits weitgehend ausgeschöpft werden (z. B. München und Umland) oder andererseits, dass es sich um wirtschaftlich prosperierende Regionen mit einer dörflich/kleinstädtisch geprägten Siedlungsstruktur handelt, in denen nur wenige Leerstände oder Brachflächen vorhanden sind (z. B. Emsland, Oberbayern, Schwaben). Aber auch Regionen, die ein sehr hohes Innenentwicklungspotenzial aufweisen - wie die Altmark - können ihre Siedlungs- und Verkehrsflächenentwicklung nur in geringem Umfang reduzieren. Dies hängt damit zusammen, dass die Siedlungsflächennachfrage dort weitgehend zum 
Abb.4.11 Reduzierung der Flächeninanspruchnahme in Gemeinden und Städten bis 2030 durch die Maßnahme „Stärkung der Innenentwicklung“"

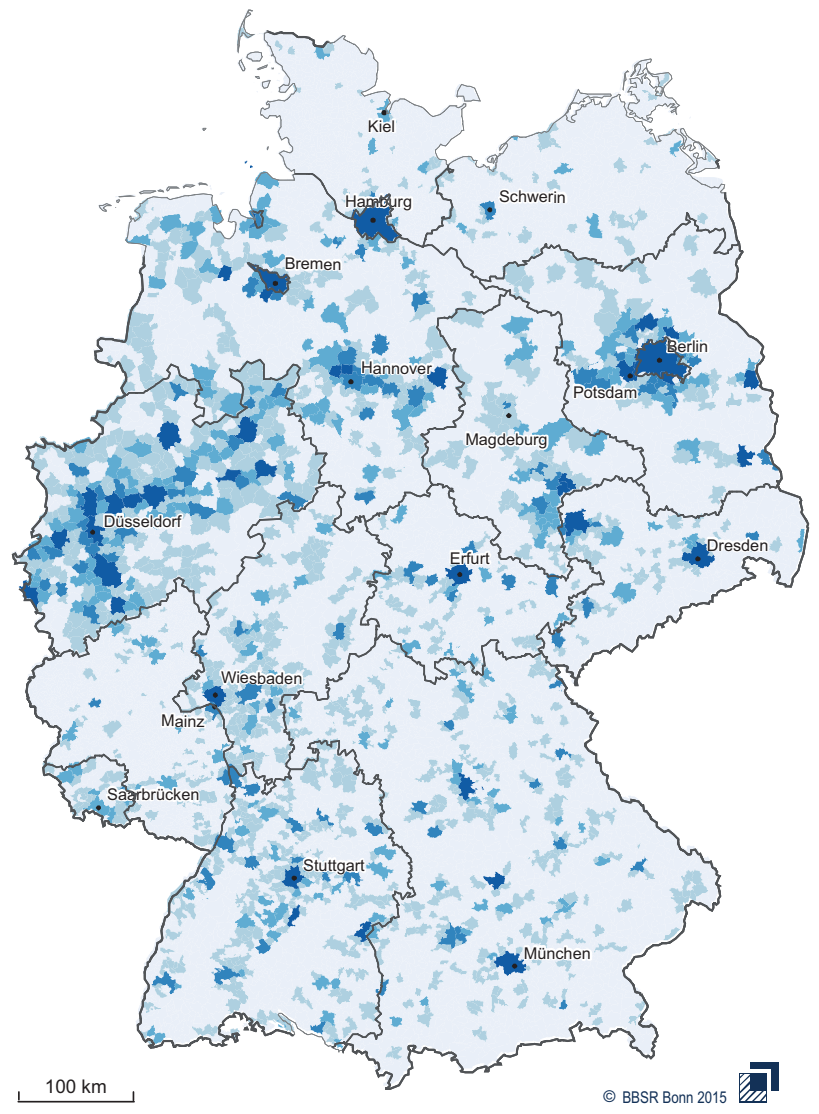

Reduzierung der Flächenneuinanspruchnahme bis 2030 durch die Maßnahme "Stärkung der Innenentwicklung" in ha

$>0$ bis unter -25

- 25 bis unter -75

- 75 bis unter -125

-125 bis unter -250

-250 und mehr

Datenbasis: Laufende Raumbeobachtung des BBSR; GWS Osnabrück 2012, Destatis 2014, BBSR-Studie Innenentwicklungspotenziale in Deutschland; Berechnungen des BBSR

Geometrische Grundlage: BKG/BBSR,Gemeinden, 31.12.2012 Bearbeitung: R. Goetzke 
Erliegen gekommen ist und die vorhandenen Potenziale nicht genutzt werden können. Erholungsflächen und vor allem Verkehrsflächen (z. B. Bau der A 14), die ebenfalls zur Siedlung- und Verkehrsfläche zählen, werden durch die Maßnahme nicht beeinflusst und tragen dort weiterhin zur Flächeninanspruchnahme bei.

Abb. 4.11 zeigt, dass in absoluten Zahlen die Maßnahme ,Stärkung der Innenentwicklung“ vor allem in Großstädten mit hohem Brachflächen- oder Nachverdichtungspotenzial wirken kann (z. B. Berlin, Hamburg, Leipzig, Dresden, Köln, Dortmund etc.). Teilweise ist auch das Umland dieser Städte mit entsprechenden Potenzialen ausgestattet. Dies ist vor allem im Umland von Berlin der Fall, während im Umland anderer Großstädte - wie Hamburg oder Stuttgart - die Siedlungsflächenentwicklung nur in geringem Umfang durch Innenentwicklung gedeckt werden kann.

Auch wenn die mengenmäßig größten Möglichkeiten zur Reduzierung der Flächeninanspruchnahme durch Innenentwicklung in Kernstädten und verdichteten Regionen liegen, ist die Umsetzung der Maßnahme gerade im ländlichen Raum und in Klein- und Mittelstädten wichtig. Denn Innenentwicklung wirkt der Entleerung von Ortszentren und der Verödung von Quartieren entgegen und trägt zum Erhalt lebendiger Dörfer und Städte bei. Gleichzeitig stellen die Innenentwicklung und das in diesem Zusammenhang notwendige Flächenmanagement gerade kleine und mittlere Städte mit eingeschränkten personellen und finanziellen Ressourcen vor besondere Herausforderungen.

\subsubsection{Ausschöpfung baulicher Dichte im Neubau}

\section{Annahmen und Spezifikation der Maßnahme}

Die Siedlungsflächennachfrage wird in dieser Maßnahme durch die Anhebung der baulichen Dichte im Neubau verringert. Zur Berechnung der baulichen Dichte in der Referenzprojektion wird aus der projizierten Zahl der fertiggestellten Wohnungen und der projizierten Gebäude- und Freiflächenentwicklung ein Geschossflächenindex berechnet. Ist der berechnete Geschossflächenindex niedriger als der Durchschnitt des gleichen siedlungsstrukturellen Kreistyps, wird er bis auf diesen Durchschnittswert angehoben. Dabei ist der Geschossflächenindex in kreisfreien Großstädten um das 2,5-fache höher als in städtischen Kreisen und dort wiederum doppelt so hoch wie in ländlichen Kreisen mit Verdichtungsansätzen und in dünn besiedelten ländlichen Kreisen. ${ }^{8}$ Durch die Orientierung an Durchschnittswerten der baulichen Dichte für siedlungsstrukturelle Kreistypen wird vermieden, dass aus städtebaulichen Gesichtspunkten „unpassend“ dicht gebaut wird (z. B. Geschosswohnungsbau in stark ländlich geprägten Gebieten).

\footnotetext{
${ }^{8}$ Vgl. Raumabgrenzungen der laufenden Raumbeobachtung des BBSR: https://www.bbsr.bund. de/BBSR/DE/Raumbeobachtung/Raumabgrenzungen/deutschland/kreise/Kreistypen4/kreistypen node.html (zitiert am 13.02.2020).
} 


\section{Auswirkungen der Maßnahme}

Durch die Ausschöpfung der baulichen Dichte im Neubau kann die tägliche Flächeninanspruchnahme im Zeitraum 2026-2030 von 45 ha/Tag in der Referenzprojektion auf 41 ha/ Tag gesenkt werden (vgl. Abb. 4.12). Der Rückgang der Flächenneuinanspruchnahme wird von der Gebäude- und Freiflächenentwicklung getragen (15 ha/Tag), auf die diese Maßnahme abzielt. Ebenso ist die Verkehrsflächenentwicklung leicht rückläufig (12,4 ha/ Tag), da durch die kompaktere Bauweise im Neubau weniger Erschließungsstraßen benötigt werden. Die Maßnahme wirkt komplementär zur Siedlungsentwicklung dort am stärksten, wo diese hoch ist. In Regionen, in denen bereits heute stark verdichtet gebaut wird, bringt die Maßnahme keinen zusätzlichen Effekt. Die Außenentwicklung ist in diesen Städten trotz hoher Wohnungsnachfrage gering, da bereits heute vor allem im Innenbereich stark verdichtet gebaut wird (z. B. München).

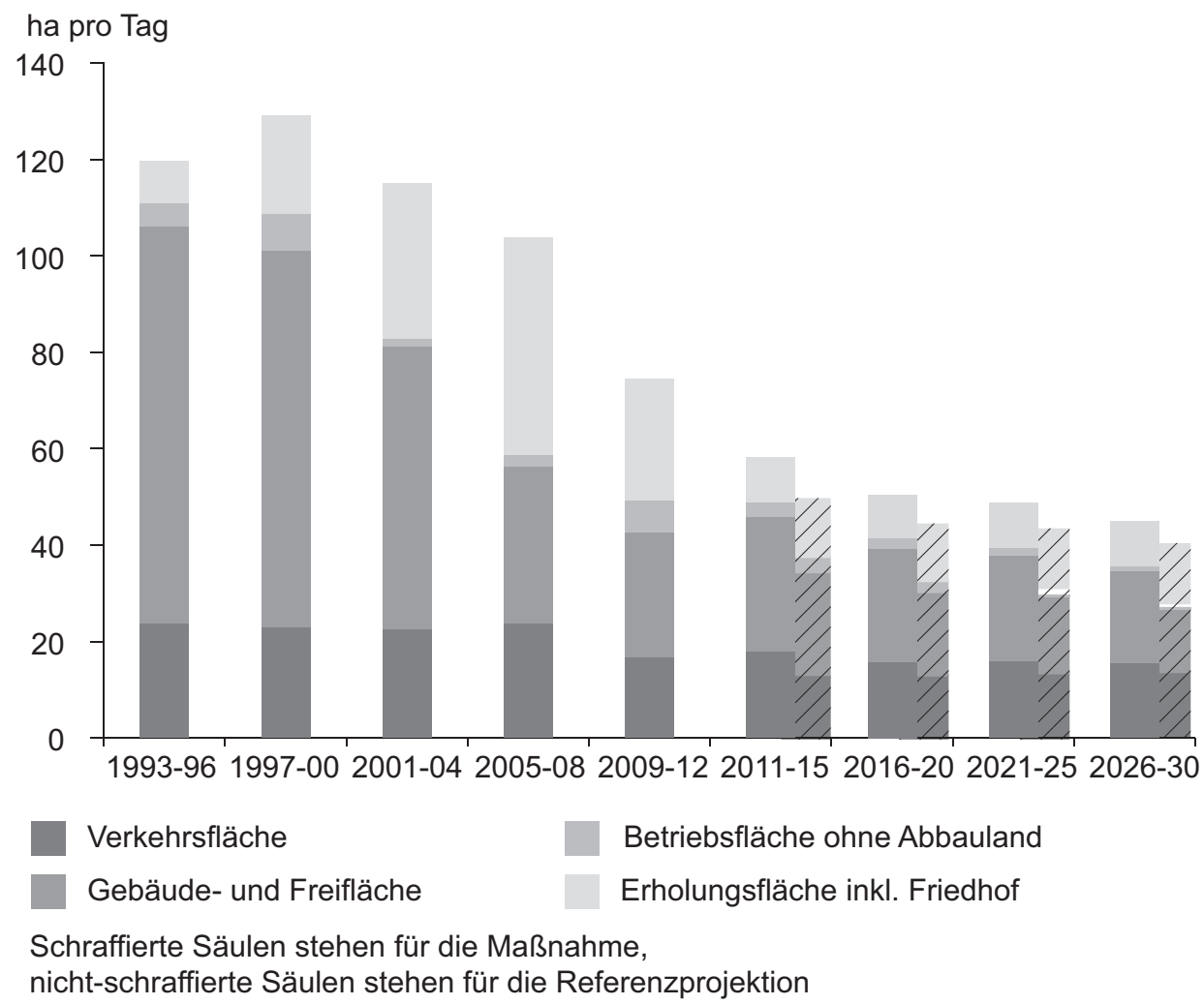

Abb. 4.12 Veränderung der Siedlungs- und Verkehrsfläche 1993 bis 2030 für die Szenarien „Referenzprojektion“ und „Ausschöpfung baulicher Dichte im Neubau“ (schraffierte Säulen stehen für die Maßnahme, nicht-schraffierte Säulen stehen für die Referenzprojektion) 


\subsubsection{Reduzierung der Flächeninanspruchnahme durch Verkehr}

\section{Annahmen und Spezifikation der Maßnahme}

Für die Umsetzung der Maßnahme „Reduzierung der Flächeninanspruchnahme durch Verkehr" im Modell werden mehrere Annahmen hinsichtlich des Rückbaus und der effizienteren Nutzung von Verkehrsflächen getroffen. Es findet ein Rückbau bereits stillgelegter Verkehrsflächen statt, wobei unter „Rückbau“ nicht zwangsläufig die physische Entfernung aller Elemente der Verkehrsinfrastruktur zu verstehen ist, sondern die endgültige Aufgabe der Nutzung. Beispielsweise können Bahntrassen erhalten bleiben, da sie sich oft zu Sonderstandorten für seltene Pflanzen entwickeln können. Im Modell wird eine solche Fläche dann nicht mehr als Verkehrsfläche weitergeführt. Des Weiteren findet eine Konzentration auf den Erhalt und Ausbau, statt auf den Neubau von Verkehrsinfrastruktur statt. Dabei wird davon ausgegangen, dass weniger Verkehrsprojekte umgesetzt werden als in der Referenzprojektion und dass vor allem in unzerschnittenen, verkehrsarmen Räumen zusätzliche Verkehrsprojekte vermieden werden. Der innerstädtische Flächenbedarf für Verkehrsflächen wird reduziert, indem der Ausbau verringert wird und in Regionen mit langfristig rückläufigem Verkehrsaufkommen unterausgelastete Infrastruktur zurückgebaut wird. Um die vorhandene Verkehrsinfrastruktur effizienter zu nutzen und Neubau zu vermeiden, wird die Siedlungsflächenentwicklung an leistungsfähigen Verkehrs- und Infrastrukturorten/-trassen konzentriert. Damit erhöht sich die Siedlungsentwicklung in Gebieten mit guter verkehrlicher Erreichbarkeit (Autobahnanschlüsse, Fernbahnhöfe, Flughäfen), während sie in schlechter erreichbaren Gebieten zurückgeht.

\section{Auswirkungen der Maßnahme}

Die Maßnahme „Reduzierung der Flächeninanspruchnahme durch Verkehr“ führt zu einem Rückgang der täglichen Flächeninanspruchnahme auf 43,5 ha (vgl. Abb. 4.13). Damit werden bis 2030 knapp 23.000 ha weniger Fläche in Anspruch genommen (vgl. Tab. 4.6). Darin enthalten ist zu einem geringen Anteil ein Rückgang der Grün- und Erholungsflächenentwicklung. Durch den geringeren Ausbau der Verkehrsinfrastruktur werden auch weniger Ausgleichs- und Ersatzmaßnahmen benötigt, die sonst teilweise als städtische Grünfläche realisiert werden. In einigen Regionen kommt es durch die Maßnahme zu einer Verlagerung der Siedlungs- und Verkehrsflächenentwicklung von Gemeinden mit schlechterer, zu Gemeinden mit besserer verkehrlicher Anbindung an Infrastrukturknotenpunkte. Dies lässt sich beispielsweise im Umland von Berlin und Hamburg, in Ostfriesland oder dem südlichen Sachsen-Anhalt beobachten.

\subsubsection{Rückzug aus der Fläche}

\section{Annahmen und Spezifikation der Maßnahme}

Die Maßnahme besteht aus einer Kombination aus dezentraler Konzentration und Rückbau in schrumpfenden peripheren ländlichen Räumen. Dabei findet eine Konzentration 
Abb.4.13 Veränderung der Flächeninanspruchnahme durch die Maßnahme „Reduzierung der Flächeninanspruchnahme durch Verkehr" gegenüber der Referenzprojektion

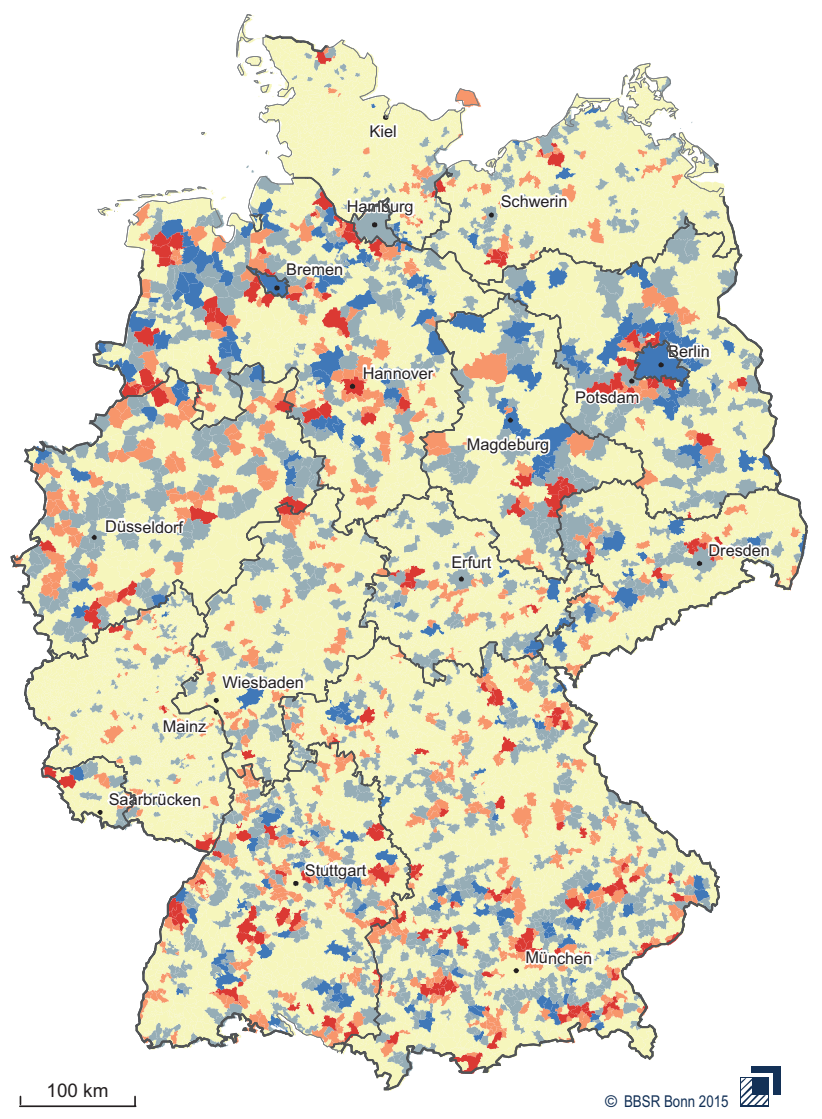

Veränderung der Flächeninanspruchnahme bis 2030 durch die Maßnahme "Reduzierung der Flächeninanspruchnahme durch Verkehr" gegenüber der Referenzprojektion

mehr als -25 ha

-25 bis unter -5 ha

-5 bis unter 5 ha

5 bis unter 25 ha

mehr als 25 ha

Datenbasis: Laufende Raumbeobachtung des BBSR; GWS Osnabrück 2012 Destatis 2014, Erreichbarkeitsmodell des BBSR 2014; ATKIS Basis DLM, BKG; Berechnungen des BBSR

Geometrische Grundlage: BKG/BBSR, Gemeinden, 31.12.2012

Bearbeitung: R. Goetzke 
der Siedlungsentwicklung auf mittel- und oberzentrale Orte statt (ohne Unterzentren mit Teilfunktionen von Mittelzentren). Neben einer Konzentration der Entwicklung im ländlichen Raum führt diese Maßnahme zu einer Entlastung von Großstadtregionen, da die Mittelzentren in deren Randbereich ebenfalls an Bedeutung gewinnen. In ländlich-peripheren Regionen mit Schrumpfungstendenzen findet Rückbau und Entsiegelung bei gleichzeitiger Stärkung des Bestands statt. Für die Umsetzung der Maßnahme werden Regionen mit hohen Leerstandsquoten identifiziert und je nach siedlungsstrukturellem Kreistyp die Leerstände reduziert. Dabei wird nochmals unterschieden zwischen ländlich-peripheren und verstädterten Gemeinden innerhalb der Kreise. Für diese Maßnahme bedarf es einer Senkung des (modellinternen) Aufwands für den Rückbau von Gebäudeund Freifläche, wodurch die Wahrscheinlichkeit im Modell für eine Umwandlung in die Kategorie Grün- und Erholungsfläche steigt. In dieser Maßnahme ist ebenfalls enthalten, dass der Rückbau in Gebieten mit erhöhtem Schadenspotenzial (Hochwasser, Lawinen) wahrscheinlicher ist als in Regionen ohne diese Gefährdung.

\section{Auswirkungen der Maßnahme}

Aufgrund der Maßnahme kann die Flächenneuinanspruchnahme bis 2030 auf weniger als 42 ha pro Tag sinken. Maßgeblich hierfür ist der Rückbau an Gebäude- und Freifläche von ca. 23.000 ha (vgl. Tab. 4.6). Die tägliche Zunahme an Gebäude- und Freifläche sinkt von 19,5 ha in der Referenzprojektion auf 16 ha (vgl. Abb. 4.14).

In der räumlichen Darstellung der Ergebnisse (vgl. Abb. 4.15) wird die Wirkung der Maßnahme deutlich: In peripheren ländlichen Gebieten, v. a. in Sachsen-Anhalt, Brandenburg und Mecklenburg-Vorpommern, findet ein deutlicher Rückgang der Neuinanspruchnahme durch Siedlungs- und Verkehrsflächen statt - regional bis zum NettoRückbau von Siedlungsfläche. Aufgrund der dezentralen Konzentration mit Stärkung des Zentrale-Orte-Systems erhöht sich in Gemeinden mit zentralörtlichen Funktionen die Siedlungsflächenzunahme. Dieser Konzentrationsprozess wird zum einen deutlich in ländlich geprägten Regionen in Bayern, Baden-Württemberg, Rheinland-Pfalz und Niedersachsen, wo eine Verlagerung der Siedlungsentwicklung aus benachbarten ländlichen Gemeinden ohne zentralörtliche Funktionen stattfindet. Zum anderen treten Mittelzentren im Einzugsbereich von Großstadtregionen deutlich hervor, die zunehmend Teile von deren Funktionen übernehmen. Damit erfolgt eine Verlagerung der Siedlungsentwicklung aus den Großstadtregionen in benachbarte Mittelzentren. Diese werden zusätzlich durch eine Verlagerung der Siedlungsflächenentwicklung aus dem angrenzenden ländlichen Raum gestärkt. Dies ist vor allem im Umland von Berlin, Frankfurt/Mainz/ Wiesbaden, Hamburg, Düsseldorf, Köln, Bonn und Dresden zu beobachten. Im Ruhrgebiet wird durch die Maßnahme ein Teil der Siedlungsflächenentwicklung von den Großstädten mit deutlicherem Zuwachs in der Referenzprojektion (z. B. Essen, Dortmund) in die benachbarten Zentren sowie in Städte im erweiterten Umland (südliches Münsterland) verlagert. Dies hängt mit der Stärkung dieser Zentren durch die 


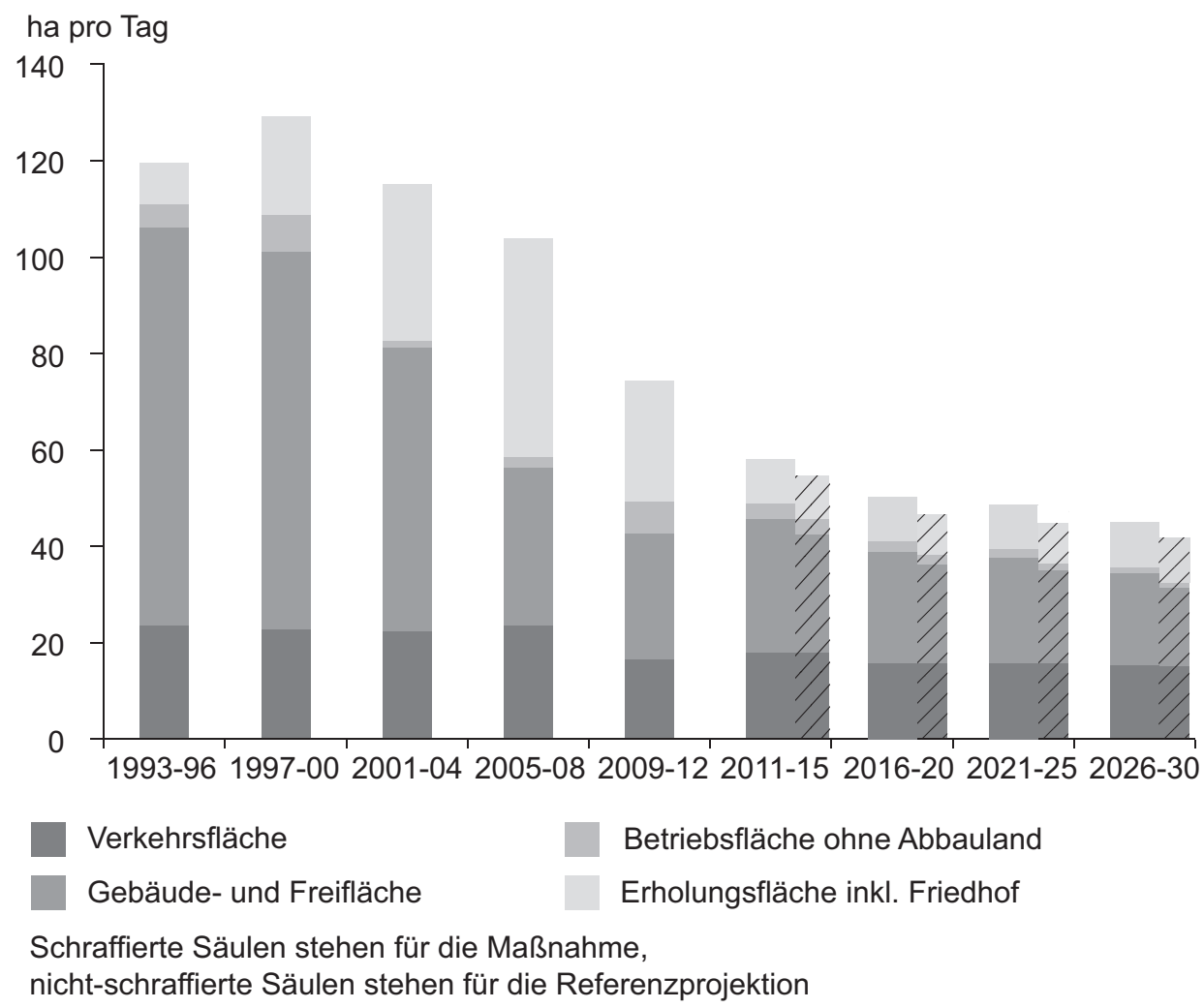

Abb. 4.14 Veränderung der Siedlungs- und Verkehrsfläche 1993 bis 2030 für die Szenarien „Referenzprojektion“ und „Rückzug aus der Fläche“ (schraffierte Säulen stehen für die Maßnahme, nicht-schraffierte Säulen stehen für die Referenzprojektion)

dezentrale Konzentration zusammen. Hierdurch werden diese Städte nicht nur gegenüber dem Umland attraktiver und erhalten mehr Zuzug, sondern werden auch gegenüber den benachbarten Zentren gestärkt.

\subsubsection{Erhalt und Entwicklung innerstädtischer Freiflächen}

\section{Annahmen und Spezifikation der Maßnahme}

Bei der Anpassung von Siedlungen an den Klimawandel ist neben der Berücksichtigung von zunehmendem Hochwasserrisiko die steigende Anzahl an Extremwetterlagen zu beachten. Wenn die Anzahl an Hitzetagen weiter zunimmt, gilt es, Maßnahmen zu ergreifen, die deren Auswirkungen im Siedlungsraum abmildern. Der „Erhalt und die Entwicklung innerstädtischer Freiflächen“ ist eine Maßnahme, die dazu beiträgt, das Wohlbefinden in den Städten bei steigender Hitzebelastung zu verbessern, da sie die Menge und die Erreichbarkeit urbaner Grünflächen erhöht. Die Maßnahme beinhaltet, 
Abb.4.15 Veränderung der Flächenneuinanspruchnahme durch Siedlung und Verkehr aufgrund der Maßnahme „Rückzug aus der Fläche" gegenüber der Referenzprojektion

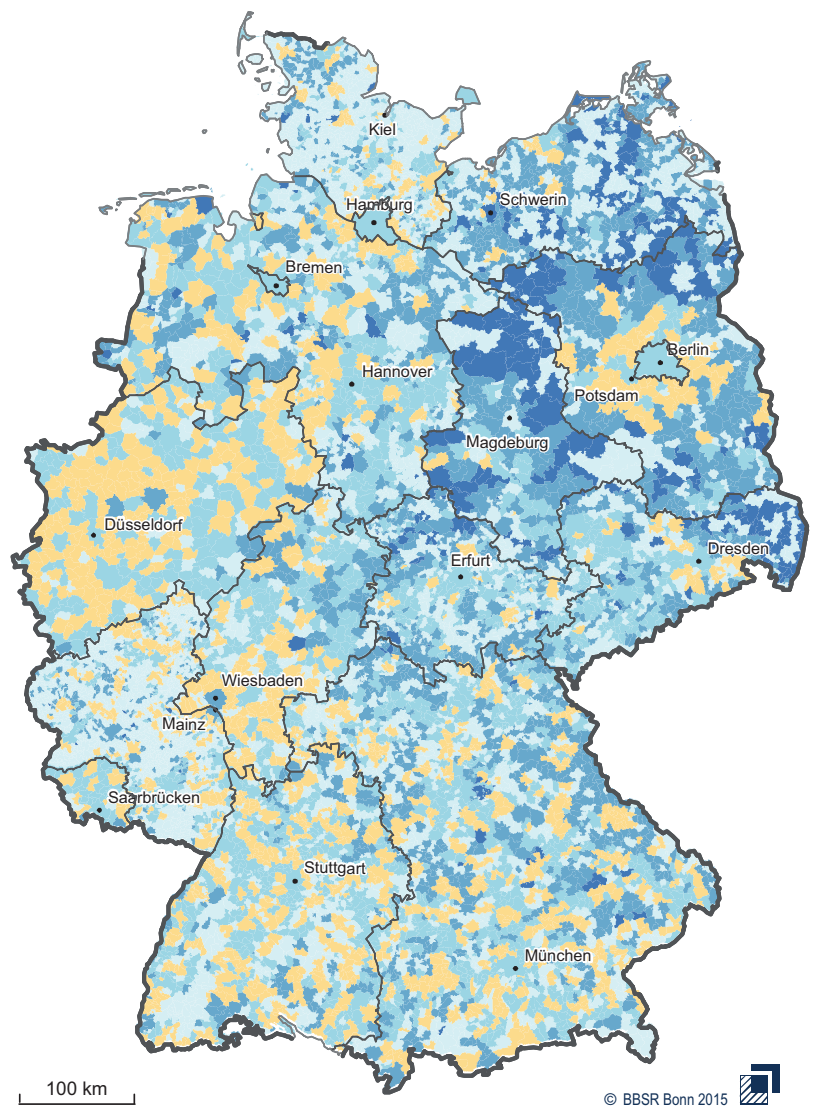

Veränderung der Flächenneuinanspruchnahme bis 2030 durch die Maßnahme "Rückzug aus der Fläche" gegenüber der Referenzprojektion

\section{Zunahme}

keine Veränderung

Reduzierung um $<50 \%$

Reduzierung um $>50 \%$

Rückbau

Datenbasis: Laufende Raumbeobachtung des BBSR; GWS Osnabrück 2012, Destatis 2014, BBSR-Studie Entwicklung von Wohnungsleerständen 2014; Berechnungen des BBSR

Geometrische Grundlage: BKG/BBSR, Gemeinden, 31.12.2012 Bearbeitung: R. Goetzke 
dass bestehende innerstädtische Grünflächen erhalten bleiben, indem der Aufwand zur Bebauung von Grün- und Erholungsflächen erhöht und die Nachverdichtungsrate im Verhältnis zur Referenzprojektion reduziert wird. Im Neubau werden Luftaustauschkorridore und qualitätsvolle Grünflächen berücksichtigt. Durch die Stärkung raumplanerischer Instrumente zum Siedlungsklimaschutz, Freiraumschutz, regionale Grünzüge und Grünzäsuren sowie der Landschaftsplanung bleiben stadtregionale Freiraumfunktionen sowie grüne und blaue Strukturen erhalten. Im Stadtraum werden neue Siedlungsflächen geschaffen (z. B. auf Brachen). Hierfür wird die Erholungsfläche je Einwohner erhöht und vor allem dort verortet, wo bislang Defizite hinsichtlich der Grünausstattung bestehen, und der Aufwand zur Umnutzung von Brachen in Grünflächen abgesenkt. In Kreisen mit hohen Leerstandsquoten und Bevölkerungsrückgang findet Rückbau, Entsiegelung und Konzentration gebauter Strukturen statt.

\section{Auswirkung der Maßnahme}

Die Wirkung dieser Maßnahme wird mit dem Indikator „Flächeninanspruchnahme in wärmebelasteten Gebieten“" bewertet. Dieser Indikator setzt sich aus zwei Teilindikatoren zusammen, die gleichgewichtet in den Gesamtindikator einfließen. Dabei handelt es sich einerseits um den Anteil der Flächenneuinanspruchnahme (Wohnbebauung) in wärmebelasteten Gebieten und andererseits um den Anteil der Siedlungsflächen im Jahr 2030 (Wohnbebauung) innerhalb einer Distanz von $500 \mathrm{~m}$ um grüne und blaue Strukturen (urbane Grün- und Erholungsflächen, Wald, Feuchtgebiete, Wasser). Die tägliche Flächeninanspruchnahme liegt im Zeitraum 2026 bis 2030 bei Umsetzung der Maßnahme „Erhalt und Entwicklung innerstädtischer Freiflächen“ bei 47 ha/Tag und damit nur leicht oberhalb der Referenzprojektion. Dies hängt in erster Linie mit einem höheren Bedarf an Erholungsflächen zusammen (13 ha/Tag). Die Gebäude- und Freiflächenentwicklung ist aufgrund des Rückbaus von Leerständen geringer als in der Referenzprojektion und liegt im Zeitraum 2026 bis 2030 bei etwa 18 ha/Tag (vgl. Abb. 4.16). ${ }^{9}$ Die Verkehrsflächenentwicklung wird durch die Umsetzung der Maßnahme nur leicht erhöht. Dies hängt mit der Verlagerung der Gebäude- und Freiflächenentwicklung an die Siedlungsränder zusammen, da Freiflächen im Innenbereich stärker in Grünflächen umgewandelt werden als in der Referenzprojektion. Zudem wird eine insgesamt lockerere Bebauung angestrebt, was längere Erschließungsstraßen zur Folge hat.

Die Maßnahme ist vor allem in Regionen wirksam, die einen hohen Brachflächenanteil, bzw. Leerstand bei geringem Flächendruck haben (vgl. Abb. 4.17). Dort ergeben sich größere Handlungsspielräume für die Schaffung neuer Grünflächen bspw. durch Rückbau. Dies ist vor allem in Teilen Sachsens, im südlichen Brandenburg sowie in Teilen Thüringens und Sachsen-Anhalts der Fall. Insgesamt erhöht sich allerdings durch

\footnotetext{
${ }^{9}$ Gebäude- und Freifläche bleibt zwar im Vergleich zur Referenzprojektion konstant, doch durch den Rückbau ergibt sich ein Netto-Rückgang der Flächenentwicklung.
} 


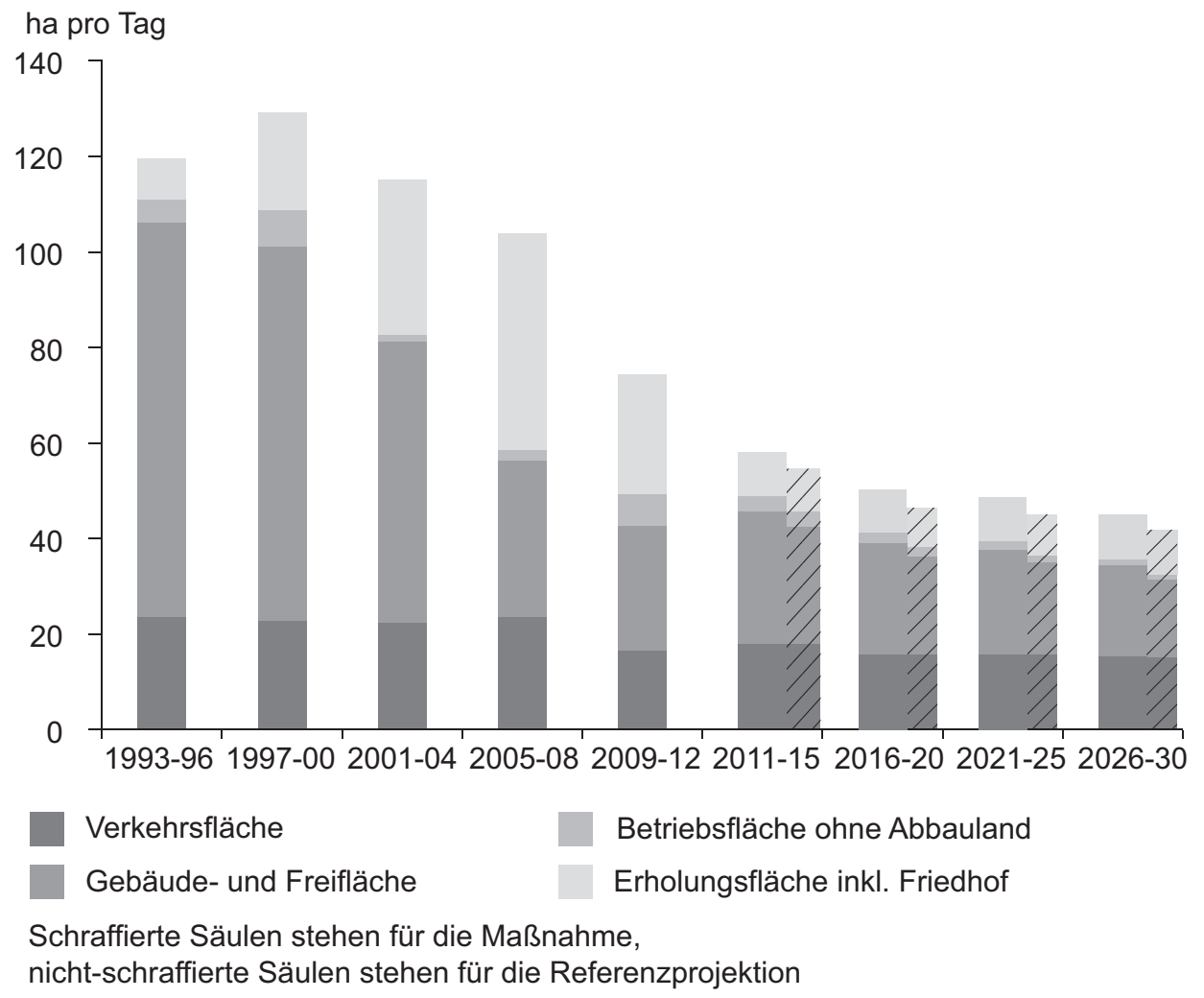

Abb. 4.16 Veränderung der Siedlungs- und Verkehrsfläche 1993 bis 2030 für die Szenarien „Referenzprojektion“ und „Erhalt und Entwicklung innerstädtischer Freiflächen“ (schraffierte Säulen stehen für die Maßnahme, nicht-schraffierte Säulen stehen für die Referenzprojektion)

die stärkere Berücksichtigung des Freiraums der Bedarf an neuer Siedlungs- und Verkehrsfläche leicht, da durch die konsequente Anwendung der Maßnahme die Möglichkeiten der Innenentwicklung eingeschränkt werden und allgemein eine lockerere Bebauung angestrebt wird. Dies hat für einige Regionen zur Konsequenz, dass der Indikator „Flächeninanspruchnahme in wärmebelasteten Gebieten“ aufgrund der höheren Gebäude- und Freiflächenentwicklung im Vergleich zur Referenzprojektion leicht zunimmt. Im Ergebnis sind die Unterschiede zwischen der Referenzprojektion und Maßnahmewirkung gering, da in vielen Regionen die positiven Effekte, die sich aus einer besseren Erreichbarkeit von erholungswirksamen grünen und blauen Strukturen ergeben, durch eine höhere Flächenneuinanspruchnahme in wärmebelasteten Gebieten ausgeglichen werden. 
a

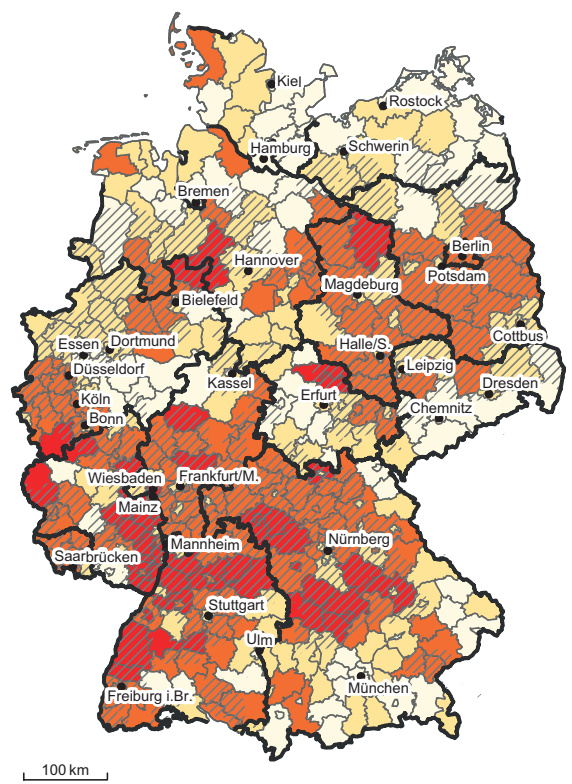

Referenzprojektion (im Vergleich zum Bundesdurchschnitt)

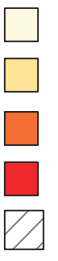

stark unterdurchschnittlich

leicht unterdurchschnittlich

leicht überdurchschnittlich

weit überdurchschnittlich

Gebiete mit Hitzebelastung

2001-2010: >= 7 heiße Tage / Jahr

Anzahl heißer Tage / Jahr steigt

bis $2091-2100$ um $>7$ b

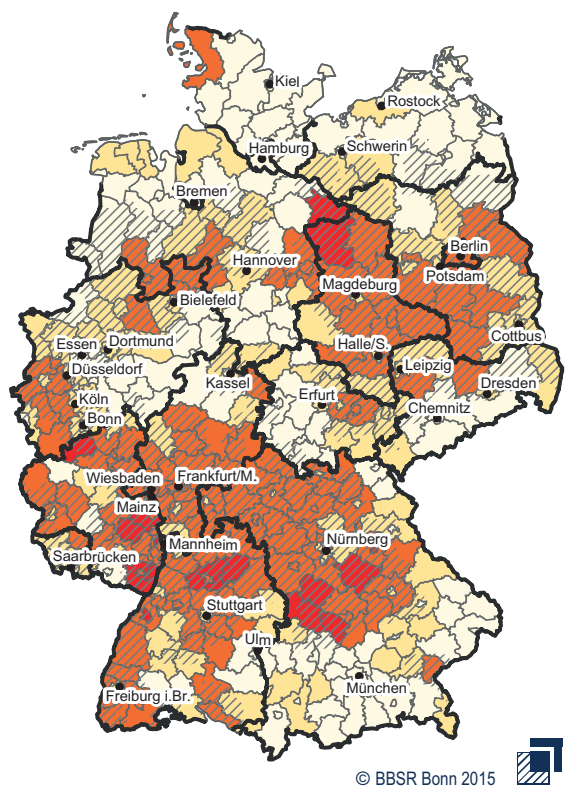

Referenzprojektion 2030 mit Maßnahme "Erhalt und Entwicklung innerstädtischer Freiflächen" (Abweichung vom Bundesdurchschnitt 2009)

Datenbasis:

Laufende Raumbeobachtung des BBSR; GWS Osnabrück 2012; Klimaprojektion: PIK 2014; Berechnungen des BBSR

Geometrische Grundlage: BKG/BBSR, Kreise, 31.12.2008 Bearbeitung: R. Goetzke

Abb. 4.17 Flächeninanspruchnahme in wärmebelasteten Gebieten (Simulation der Siedlungsund Verkehrsflächenentwicklung bis 2030 und Berücksichtigung der Entwicklung der Hitzetage bis 2100). Vergleich zwischen Referenzprojektion (links) und Maßnahme „Erhalt und Entwicklung innerstädtischer Freiflächen“ (rechts)

\subsubsection{Stärkung des ÖPNV}

\section{Annahmen und Spezifikation der Maßnahme}

Die Maßnahme „Stärkung des ÖPNV“ zielt auf die verbesserte Anbindung neuer Siedlungsflächen an den ÖPNV ab. Damit soll der Umstieg vom MIV auf den ÖPNV erleichtert, Fahrstrecken reduziert und eine kompakte und effiziente Siedlungsstruktur 
erreicht werden. Ziel dieser Maßnahme ist die Reduzierung von $\mathrm{CO}_{2}$-Emissionen im Verkehrssektor. Die zentralen Modellannahmen umfassen Verbesserung der ÖPNVErreichbarkeit von ober- und mittelzentralen Orten und die stärkere Gewichtung dieser Erreichbarkeit bei der Siedlungsentwicklung. Konkret wird im Modell beispielsweise die Erreichbarkeit von zentralen Orten mit dem ÖPNV höher gewichtet als die mit dem motorisierten Individualverkehr, und unter der Annahme, dass es zu einer Angebotsverbesserung kommt, auch von Ortszentren und Haltestellen entferntere Siedlungsflächen in die Gewichtung mit einbezogen. Da zudem eine hohe Siedlungsdichte die Erreichbarkeit von Orten und Einrichtungen des täglichen Bedarfs erhöht, wird im Modell die Bedeutung der Siedlungsdichte für die Wohnstandortwahl heraufgesetzt. Das bedeutet, bereits verdichtete Orte sind für die Standortwahl im Modell attraktiver als gering verdichtete. Das führt zu einer stärkeren Konzentration der Siedlungsentwicklung in verdichteten Regionen und somit zu kürzeren Wegen und besserer ÖPNV-Auslastung.

Um die Wirkung der Maßnahme abschätzen zu können, wird der Indikator ,Verkehrliche Erschließung neuer Siedlungsflächen“ verwendet. Der Indikator misst die ÖPNV-Erreichbarkeit mittel- und oberzentraler Orte von neuen Siedlungsflächen (Wohnbebauung). Als Datengrundlage dient eine Karte der Erreichbarkeit von Mittel- und Oberzentren aus dem Erreichbarkeitsmodell des BBSR (Abb. 4.18). ${ }^{10}$

Im Durchschnitt benötigt man von den bestehenden Siedlungsflächen aus etwa $30 \mathrm{~min}$ bis zum nächsten Zentrum eines mittel- oder oberzentralen Ortes. Regionale Unterschiede der ÖPNV-Erreichbarkeit sind deutlich zu erkennen (vgl. Abb. 4.18). Defizite sind vor allem im ländlich geprägten Norden und Osten (v. a. Mecklenburg-Vorpommern) zu erkennen, während in den stärker verdichteten Kreisen im Westen und Süden sowie in den meisten kreisfreien Städten ${ }^{11}$ die ÖPNV-Erreichbarkeit gut ist.

\section{Auswirkung der Maßnahme}

Von den Siedlungsflächen, die in der Referenzprojektion bis 2030 neu hinzukommen, sind Mittel- und Oberzentren mit dem ÖPNV im Schnitt in $28 \mathrm{~min}$ zu erreichen. Durch die Maßnahme „Stärkung des ÖPNV“ verbessert sich diese Erreichbarkeit auf 23 min. Eine deutliche Abweichung von diesem Wert ist nach Durchführung der Maßnahme nur noch in wenigen Kreisen messbar. Abb. 4.19 zeigt den Index „Verkehrliche Erschließung neuer Siedlungsflächen“, der sich aus der Abweichung der Erreichbarkeit von Mittel- und Oberzentren ausgehend von neuen Siedlungsflächen errechnet. Auf der linken Seite der Karte sind diese Abweichungen in der Referenzprojektion erkennbar. Die rechte Seite der Karte zeigt die Maßnahmenwirkung.

\footnotetext{
${ }^{10}$ Die ÖPNV-Erreichbarkeit entspricht dem Status quo. Über die zukünftige Entwicklung des ÖPNV-Netzes liegen keine Informationen vor. Daher wird die Lage neuer Siedlungsflächen anhand der heutigen ÖPNV-Erreichbarkeit bewertet.

${ }^{11}$ Berlin bildet hier eine Ausnahme, was allerdings mit dem Erreichbarkeitsmodell zusammenhängt, das als Zielorte die Zentren der Städte beinhaltet. Bei der Größe Berlins sind bis ins Zentrum erhebliche Strecken zurückzulegen.
} 


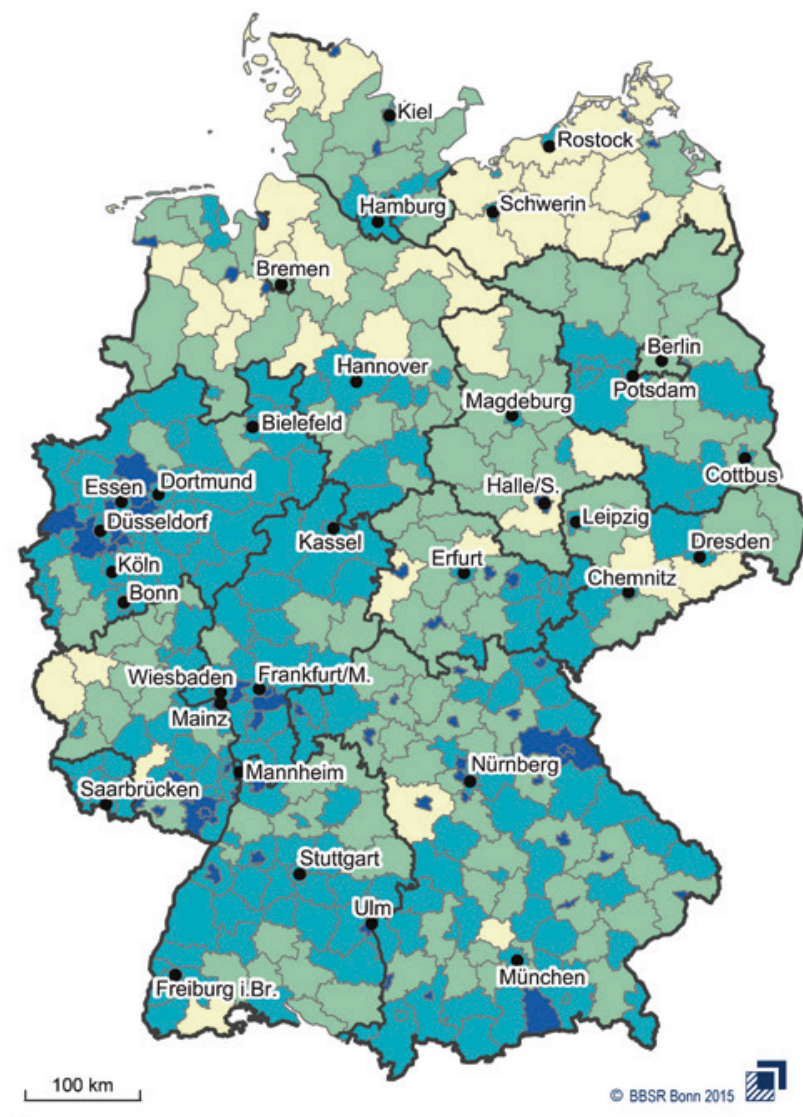

\section{ÖPNV-Erreichbarkeit von Mittel- und Oberzentren (in Minuten)}

weniger als 20

20 bis unter 30

30 bis unter 40

D 40 und mehr

\footnotetext{
Datenbasis: Laufende Raumbeobachtung des BBSR; GWS Osnabrūck 2012; Erreichbarkeitsmodell des BBSR; Berechnungen des BBSR;

Geometrische Grundlage: BKG/BBSR, Kreise, 31.12.2008

Bearbeitung: R. Goetzke
}

Abb. 4.18 Mittlere Erreichbarkeit von Mittel- und Oberzentren mit dem ÖPNV (ausgehend von bestehender Wohnbebauung 2009) 
a

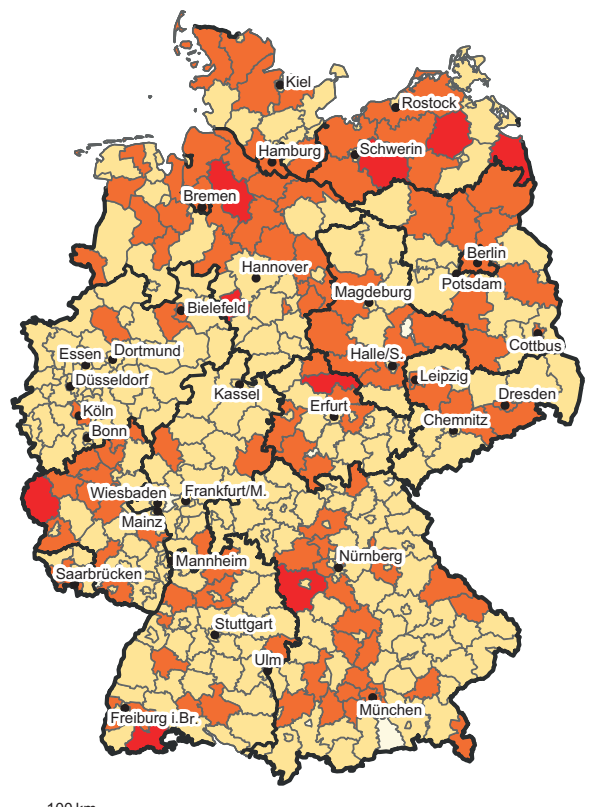

Referenzprojektion (im Vergleich zum Bundesdurchschnitt)

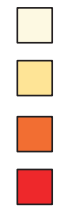

stark unterdurchschnittlich

leicht unterdurchschnittlich

leicht überdurchschnittlich

weit überdurchschnittlich b

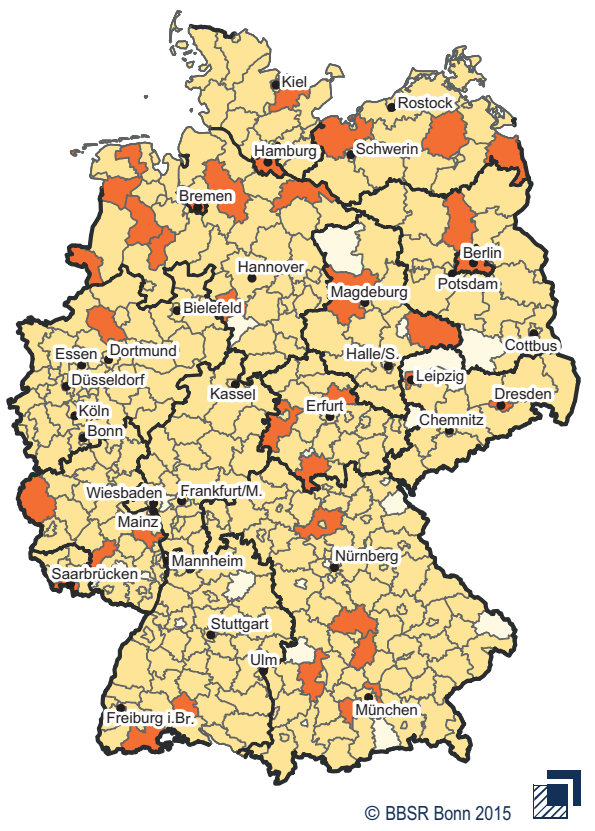

Referenzprojektion 2030 mit Maßnahme "Stärkung des ÖPNV" (Abweichung vom Bundesdurchschnitt 2009)

\footnotetext{
Datenbasis:

Laufende Raumbeobachtung des BBSR; GWS Osnabrück 2012; Errreichbarkeitsmodell des BBSR 2014

Berechnungen des BBSR;

Geometrische Grundlage: BKG/BBSR, Kreise, 31.12.2008 Bearbeitung: R. Goetzke
}

Abb.4.19 Verkehrliche Erschließung (ÖPNV) neuer Siedlungsflächen (Simulation der Siedlungs- und Verkehrsflächenentwicklung bis 2030 und Berücksichtigung der heutigen ÖVErreichbarkeit von Mittel- und Oberzentren). Vergleich zwischen Referenzprojektion Bundesdurchschnitt 2009 (links) und Maßnahme „Stärkung des ÖPNV“ (rechts)

In den meisten Regionen sind durch die Maßnahme neue Siedlungsflächen deutlich besser über den ÖPNV an Mittel- und Oberzentren angeschlossen als in der Referenzprojektion, gerade auch in ländlich-peripheren Kreisen. Ein Beispiel hierfür ist die Altmark. Dort findet durch die modellierte Maßnahme die Siedlungsentwicklung nur noch in unmittelbarer Nähe und damit guter Erreichbarkeit der wenigen Mittelzentren (Stendal und Salzwedel) statt. Hierbei gilt die Annahme einer Verbesserung des ÖPNV-Angebots. Dabei gilt es zu berücksichtigen, dass die Siedlungsentwicklung dort insgesamt verglichen mit dem Bundesdurchschnitt sehr niedrig ist und aufgrund geringer Flächenkonkurrenz die Siedlungsentwicklung auf die optimalsten Standorte (in Bezug auf die ÖPNV-Erreich- 
barkeit) ausweichen kann. Insgesamt ist die ÖPNV-Erreichbarkeit in der Altmark jedoch unterdurchschnittlich (vgl. Abb. 4.19).

Da die Maßnahme nur die räumliche Verteilung der Siedlungsentwicklung steuert und nicht deren Menge, hat sie keinen Einfluss auf die tägliche Flächenneuinanspruchnahme. Diese ist identisch mit der Siedlungs- und Verkehrsflächenentwicklung in der Referenzprojektion.

\subsubsection{Zusätzliche Ausweisung von Vorrang- und Vorbehaltsgebieten}

\section{Annahmen und Spezifikation der Maßnahme}

Bei der Maßnahme handelt es sich weniger um eine Umsetzungsmaßnahme, sondern vielmehr um die konsequente Anwendung des raumordnerischen Instrumentariums. Bestandteile dieser Maßnahme sind auch in anderen Maßnahmen enthalten. Hier werden stattdessen alle Möglichkeiten gebündelt, die der Raumordnung zur Verfügung stehen, um die Siedlungsentwicklung im Hinblick auf Anforderungen von Klimaschutz, Klimaanpassung und Natur- und Umweltschutz zu steuern. Die Maßnahme beinhaltet die Stärkung des Freiraumschutzes (z. B. durch Umwandlung von Vorbehalts- in Vorranggebiete) und den Schutz natürlicher Bodenfunktionen durch Raumordnungsgebiete. Des Weiteren werden neue Vorrang- und Vorbehaltsgebiete zum Schutz vor Naturgefahren (z. B. Hangrutschungen) ausgewiesen und Vorrang- und Vorbehaltsgebiete für erneuerbare Energien (Windenergie) erweitert.

Mit diesem Bündel an raumordnerischen Maßnahmen wird die Wahrscheinlichkeit für neue Siedlungsentwicklung in folgenden Vorrang- und Vorbehaltsgebieten deutlich herabgesetzt: Hochwasserschutz, regionale Grünzüge, Natur- und Landschaft, Landwirtschaft, Forstwirtschaft, Freiraumschutz, Siedlungsklimaschutz, Windenergienutzung, Grundwasserschutz, Bodenschutz. Außerdem werden Vorrang- und Vorbehaltsgebiete für Gebiete ausgewiesen, die ein hohes landwirtschaftliches Ertragspotenzial, einen hohen Kohlenstoffgehalt im Oberboden oder ein Hangrutschungsrisiko aufweisen.

\section{Auswirkung der Maßnahm}

Die Maßnahme führt zu einer Verlagerung der Siedlungsentwicklung. Ein klares räumliches Muster der Verlagerung ist in den Ergebniskarten nicht $\mathrm{zu}$ erkennen (vgl. Abb. 4.20). Ein solches Muster ergibt sich nicht, weil dieses Maßnahmenbündel in unterschiedliche Richtungen wirkt (Klimaschutz, Klimaanpassung, Naturschutz). Verlagerungseffekte sind beispielsweise entlang großer Flussläufe zu erkennen (Rhein, Donau), wo sich die Siedlungsentwicklung in Gemeinden in größerer Entfernung zu den Flüssen verstärkt, während sie sich in deren Nähe abschwächt (aufgrund der stärkeren Wirkung der Vorrang- und Vorbehaltsgebiete zum Hochwasserschutz).

Da die Maßnahme nur die räumliche Verteilung der Siedlungsentwicklung steuert und nicht deren Menge, hat sie keinen Einfluss auf die tägliche Flächenneuinanspruchnahme. Diese ist identisch mit der Siedlungs- und Verkehrsflächenentwicklung in der Referenzprojektion. 


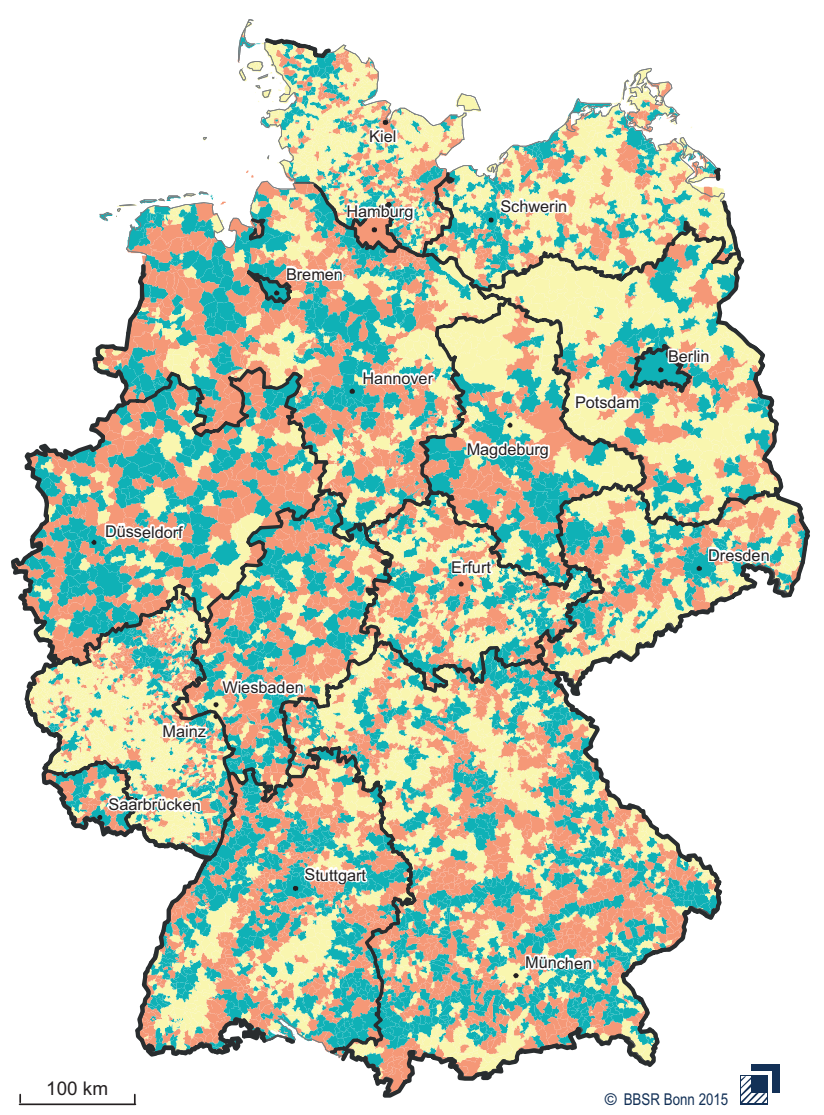

Veränderung der Flächenneuinanspruchnahme

bis 2030 durch die Maßnahme "Zusätzliche

Ausweisung von Vorrang- und Vorbehaltsgebieten" gegenüber der Referenzprojektion

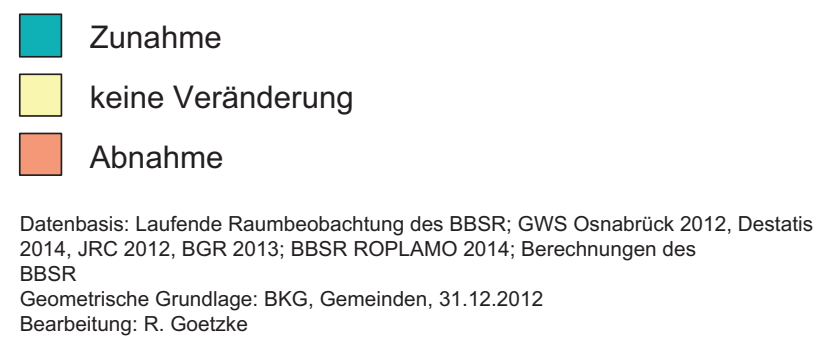

Abb.4.20 Veränderung der Flächenneuinanspruchnahme durch Siedlung und Verkehr aufgrund der Maßnahme „Zusätzliche Ausweisung von Vorrang- und Vorbehaltsgebieten“ gegenüber der Referenzprojektion 


\subsubsection{Stärkung des Hochwasserschutzes}

\section{Annahmen und Spezifikation der Maßnahme}

Die Maßnahme „Stärkung des Hochwasserschutzes“ beinhaltet vorsorgende (raumplanerische) Instrumente und zielt darauf $a b$, die Siedlungsentwicklung aus Gebieten herauszuhalten, in denen Extremhochwasser auftreten kann. Konkret werden in der Modellierung Vorranggebiete des Hochwasserschutzes stärker gewichtet als in der Referenzprojektion, was eine Erhöhung des Abwägungsaufwands darstellen soll. Zudem werden bestehende Vorbehaltsgebiete in Vorranggebiete umgewandelt. Die Hochwasserrisikomanagementrichtlinie (HWRM-RL) wird konsequent umgesetzt und zusätzlich werden Extremhochwasserbereiche $\left(\mathrm{HQ}_{\text {extrem }}\right)$ als Vorranggebiete ausgewiesen. Somit ist die Fläche, in denen aufgrund von Hochwassergefahr die Siedlungsentwicklung erschwert wird (z. B. durch höheren Begründungsaufwand oder die Vorgabe hochwasserangepasster Bauweise), deutlich größer als in der Referenzprojektion.

\section{Auswirkung der Maßnahme}

Durch die Maßnahme „Stärkung des Hochwasserschutzes“ kann die Flächeninanspruchnahme in hochwassergefährdeten Gebieten deutlich reduziert werden. Die linke Karte in Abb. 4.21 zeigt die Flächenneuinanspruchnahme durch versiegelungsrelevante Siedlungsflächen bis 2030 in durch Extremhochwasser gefährdeten Gebieten $\left(\mathrm{HQ}_{\text {extrem }}\right)$ als Abweichung der Kreiswerte vom Bundesdurchschnitt in der Referenzprojektion. In der Referenzprojektion werden die derzeit bestehenden Vorrang- und Vorbehaltsgebiete zur Steuerung der Siedlungsentwicklung berücksichtigt, im Maßnahmen-Szenario die erweiterte Kulisse an Vorranggebieten (rechte Karte). Der Indikator berechnet sich jedoch in beiden Fällen aus der Flächeninanspruchnahme in $\mathrm{HQ}_{\text {extrem }}-$ Gebieten. Damit haben die Bewertung der Referenzprojektion und die Maßnahmenwirkung die gleiche Bemessungsgrundlage.

Dabei wird deutlich, dass in den weitaus meisten Kreisen das Hochwasserrisiko durch die Ausweisung weiterer Vorranggebiete für den Hochwasserschutz deutlich reduziert wird. Ausnahmen bilden die Kreise, in denen die Siedlungsflächennachfrage sehr hoch und gleichzeitig die Menge an verfügbaren Freiflächen außerhalb hochwassergefährdeter Gebiete gering ist (Rheinland, Oberrhein, Rhein-Main). Daneben bleibt in den Kreisen die Siedlungsentwicklung in hochwassergefährdeten Bereichen hoch, in denen der weitaus größte Teil der Fläche der Kreise hochwassergefährdet ist (Nordsee). In einigen Gebieten, die vom Jahrhunderthochwasser 2013 an der Elbe und ihren Zuflüssen betroffenen waren, ist das Hochwasserrisiko auch in der Referenzprojektion vergleichsweise gering. Dies hängt mit der im Vergleich zum Bundesdurchschnitt geringen Siedlungsflächenentwicklung sowie ausreichend zur Verfügung stehenden Ausweichflächen zusammen. 
a

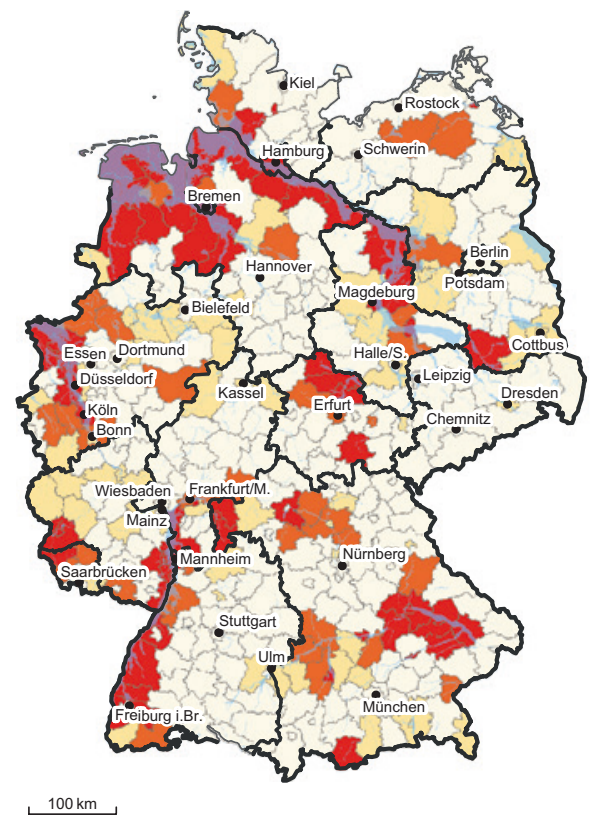

Referenzprojektion (im Vergleich zum Bundesdurchschnitt)

stark unterdurchschnittlich

leicht unterdurchschnittlich

leicht überdurchschnittlich

weit überdurchschnittlich

Gebiete mit Hochwassergefahr bei extremen Hochwasserereignissen (HQextrem) b

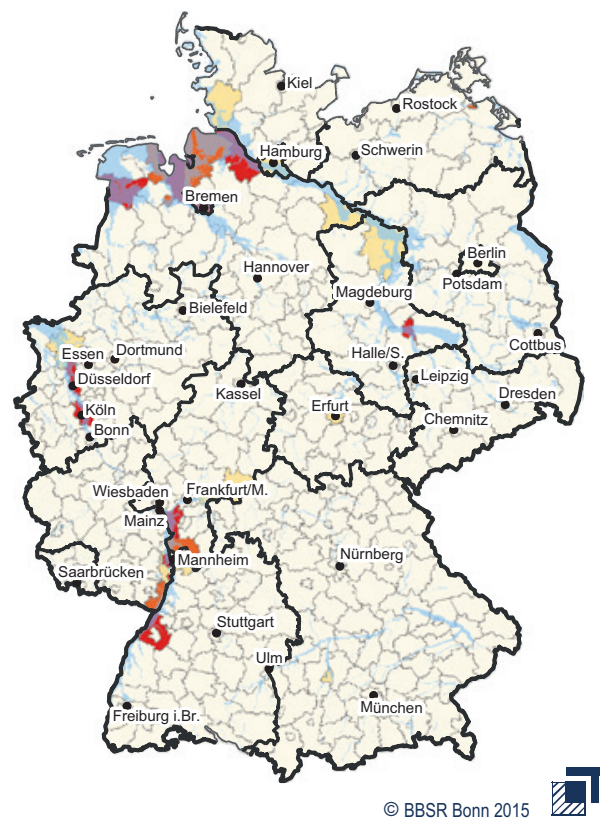

Referenzprojektion 2030 mit Maßnahme "Stärkung des Hochwasserschutzes" (Abweichung vom Bundesdurchschnitt in der Referenzprojektion)

Datenbasis:

Laufende Raumbeobachtung des BBSR; GWS Osnabrück 2012; Überflutungsbereiche nach HWRM-RL: BfG \& Zuständige Behörden der Länder 2014; ExtremeHochwasserereignisse: Hydrologischer Atlas für Deutschland, BfG 2003; Berechnungen des BBSR;

Geometrische Grundlage: BKG/BBSR, Kreise, 31.12.2008 Bearbeitung: R. Goetzke

Abb. 4.21 Flächenneuinanspruchnahme in hochwassergefährdeten Gebieten (Simulation der versiegelungsrelevanten Siedlungs-und Verkehrsflächenentwicklung bis 2030). Vergleich zwischen Referenzprojektion (links) und Maßnahme „Stärkung des Hochwasserschutzes“ (rechts)

\subsubsection{Restriktiver Freiraumschutz}

\section{Annahmen und Spezifikation der Maßnahme}

In der Maßnahme „Restriktiverer Freiraum-/Naturschutz“ wird die Schutzfunktion einiger Schutzgebietskategorien erhöht (Landschaftsschutzgebiete, Natura 2000-Gebiete, Biosphärenreservate), was die Wahrscheinlichkeit neuer Siedlungsflächenentwicklung in diesen Gebieten deutlich herabsetzt. Außerdem wird die Schutzgebietskulisse erweitert, 
indem um bestehende Schutzgebiete mit hohem Schutzstatus (Naturschutzgebiete, Nationalparks) ein Puffer gelegt wird. Zusätzlich wird die Regionalplanung in Bezug auf den Schutz von Natur und Landschaft gestärkt, bspw. durch die Umwandlung von Vorbehaltsgebieten für Natur und Landschaft und regionale Grünzüge in Vorranggebiete.

\section{Auswirkung der Maßnahme}

Mit Hilfe des Indikators „Flächeninanspruchnahme in Schutzgebieten und schutzwürdigen Landschaften“ lässt sich die Wirkung der Maßnahme räumlich darstellen (vgl. Abb. 4.22). Zunächst wird deutlich, dass es in Deutschland verschiedene „Hot Spots“ gibt, in denen vermehrt Fläche in aus Naturschutzsicht schützenswerten Gebieten für die

a

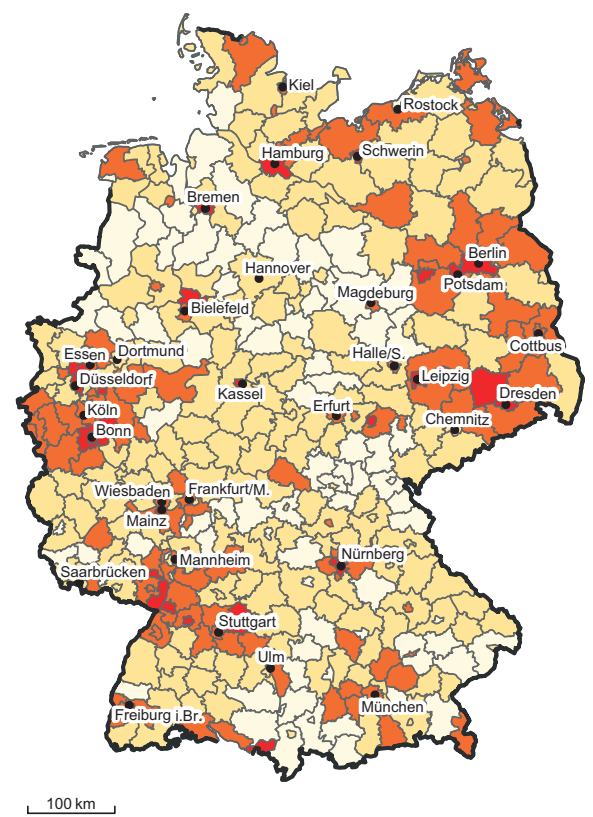

Referenzprojektion (im Vergleich zum Bundesdurchschnitt)

stark unterdurchschnittlich

leicht unterdurchschnittlich

leicht überdurchschnittlich

weit überdurchschnittlich b

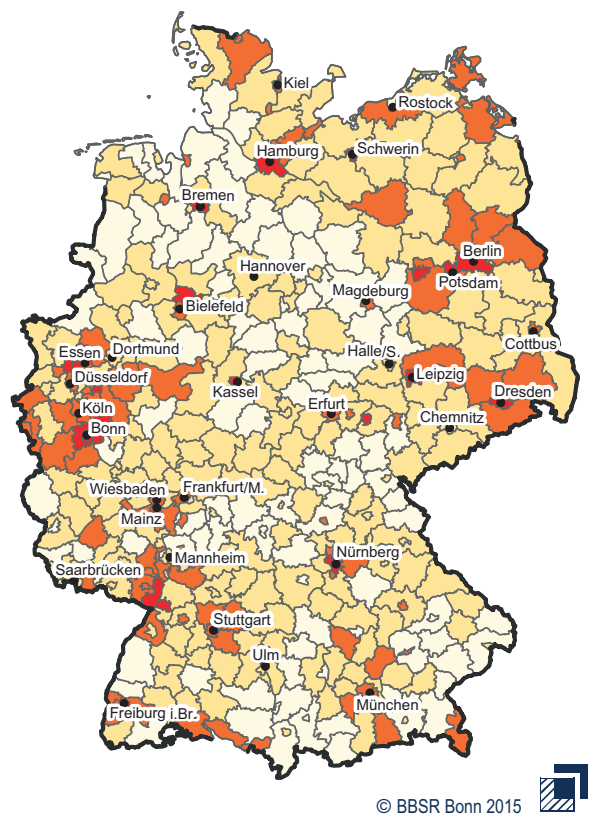

Referenzprojektion 2030 mit Maßnahme "Restriktiverer Freiraum-/Naturschutz" (Abweichung vom Bundesdurchschnitt 2009)

Datenbasis:

Laufende Raumbeobachtung des BBSR; GWS Osnabrück 2012; Schutzgebietsflächen (BfN 2011): NTP, NSG,

Natura 2000; Berechnungen des BBSR;

Geometrische Grundlage: BKG/BBSR, Kreise, 31.12.2008 Bearbeitung: R. Goetzke

Abb. 4.22 Flächeninanspruchnahme in Schutzgebieten (Simulation der Siedlungs- und Verkehrsflächenentwicklung bis 2030). Vergleich zwischen Referenzprojektion (links) und Maßnahme „Restriktiverer Freiraum-/Naturschutz“ (rechts) 
Siedlungs- und Verkehrsflächenentwicklung in Anspruch genommen wird. Dies ist beispielsweise im Rheinland, am Oberrhein, im Raum Stuttgart, im Umland von Berlin und in Teilen Sachsens der Fall. Durch die Maßnahme kommt es regional zu einem Rückgang der Flächeninanspruchnahme in Schutzgebieten und schutzwürdigen Landschaften, aber die Maßnahme scheint nicht überall zu greifen. Dies kann damit zusammenhängen, dass die Bundesländer Schutzgebietsausweisungen sehr unterschiedlich handhaben.

Zur Berechnung des Indikators wird auch die Flächeninanspruchnahme in Landschaftsschutzgebieten herangezogen, die in einigen Regionen - insbesondere im erweiterten Umland von Agglomerationen - flächenmäßig große Gebiete umfassen. Trotz der Erhöhung der Restriktion durch die Maßnahme verteilt das Modell in diesen Gebieten weiterhin Siedlungsflächen, da bei hoher Nachfrage (z. B. im Umland von Berlin oder im Rheinland) und gleichzeitig zahlreichen anderen Restriktionen (z. B. durch Hochwasserschutz) nur wenige Ausweichmöglichkeiten bestehen.

Da die Maßnahme nur die räumliche Verteilung der Siedlungsentwicklung steuert und nicht deren Menge, hat sie keinen Einfluss auf die tägliche Flächenneuinanspruchnahme. Diese ist identisch mit der Siedlungs- und Verkehrsflächenentwicklung in der Referenzprojektion.

\subsubsection{Energieerzeugung auf für Siedlungszwecke ungeeigneten nicht-agrarischen Flächen}

\section{Annahmen und Spezifikation der Maßnahme}

Um beziffern zu können, wie viel Fläche durch Freiflächen-Photovoltaikanlagen zukünftig in Anspruch genommen wird, bedarf es einer Projektion des zukünftigen Ausbaus der installierten Leistung sowie Annahmen über die Anteile von Freiflächen-Photovoltaikanlagen an diesem Ausbau. Genaue Daten hierüber liegen nicht vor. Anhand der Anlagenstammdaten der Übertragungsnetzbetreiber lassen sich jedoch grobe Schätzungen vornehmen. Die jährlich installierte Leistung an Photovoltaikanlagen ist zwischen 2005 und 2011 von 900 MW auf 7600 MW gestiegen. Im gleichen Zeitraum ist der Anteil von Freiflächen-Photovoltaikanlagen an der installierten Leistung von 6 auf $30 \%$ gestiegen (vgl. Abb. 4.23). Der Anteil der Freiflächenanlagen an der gesamten installierten Leistung (Summe aller Jahre) beträgt $21 \%$ und die in Anspruch genommene Fläche mindestens 15.600 ha.

Der Ausbaupfad des EEG zur Erzeugung von Strom aus solarer Strahlungsenergie liegt bei $2500 \mathrm{MW}$ (brutto) pro Jahr. Es werden die Annahmen getroffen, dass das Verhältnis von Freiflächenanlagen an der gesamten installierten Leistung bei $30 \%$ bleibt (Stand: 2011), der jährliche Ausbau bis 2030 genau dem Ausbaupfad folgt und der Flächenbedarf gleichbleibt.

\section{Auswirkung der Maßnahme}

Bis 2030 würden nach den oben beschriebenen Annahmen 44.460 ha Fläche für Freiflächen-Photovoltaikanlagen benötigt. $\mathrm{Ob}$ dieser Wert plausibel ist, lässt sich in 
Jährliche installierte

Anteile an

Leistung (MW)

installierter

Leistung (\%)

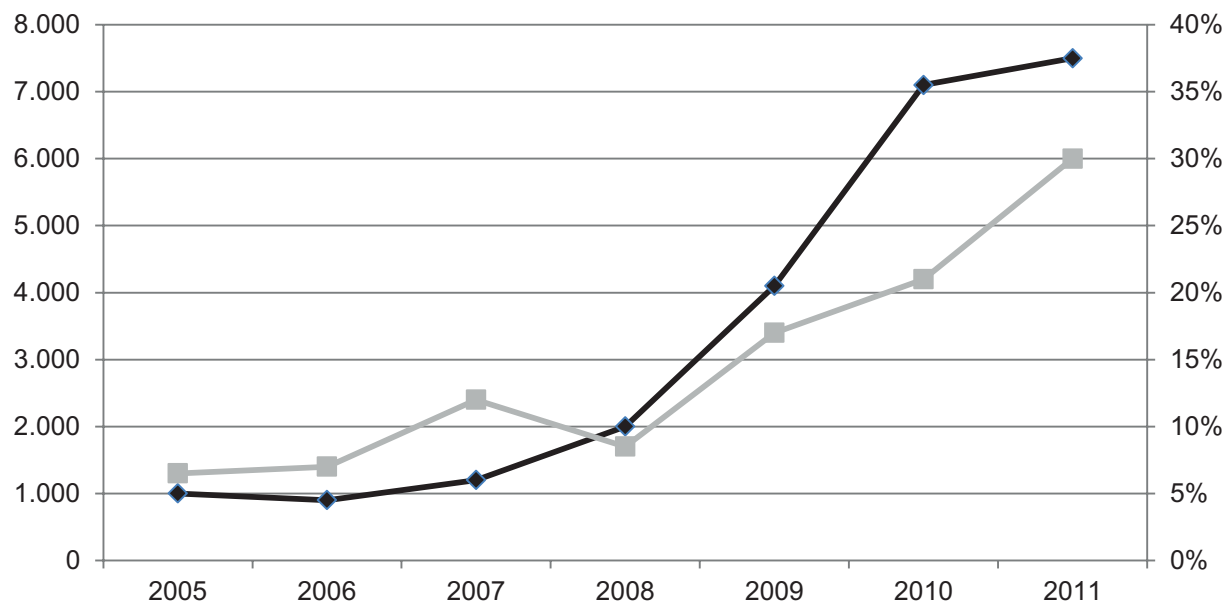

$\rightarrow$ Gesamte jährliche installierte Leistung (MW)

- Anteil Freiflächenphotovoltaik an installierter Leistung (\%)

Abb. 4.23 Photovoltaik: installierte Leistung (MW) und Anteil Freiflächen-Photovoltaikanlagen an installierter Leistung (\%) (Datengrundlage: $50 \mathrm{~Hz}$ Transmission, Amprion, Tennet TSO, TransnetBW, Berechnung R. Goetzke)

diesem Kontext nicht weiter erörtern. Möglicherweise wird weniger Fläche benötigt, da neue Anlagen effizienter werden, eine Erneuerung bestehender Anlagen durchgeführt wird (,Repowering“), die verfügbaren Flächen aufgrund der Auflagen des EEG begrenzt sind und der Ausbau möglicherweise nicht auf einem so hohen Niveau bleiben wird.

Um die Wirkung der Maßnahme abzuschätzen, wäre eine Erhebung von Flächen notwendig, die weder für Siedlungszwecke, noch für eine landwirtschaftliche Nutzung geeignet sind. Dabei handelt es sich vor allem um Konversionsflächen aus wirtschaftlicher und militärischer Nutzung. Unter Konversionsflächen aus wirtschaftlicher Nutzung fallen beispielsweise ehemalige Abbauflächen oder Deponien. In dem hier verwendeten Landnutzungsdatensatz sind etwa 17.700 ha an Abbauflächen und Deponien erfasst. Davon wären etwa 6300 ha aufgrund ihrer Ausrichtung (südlich) und ihrer Hangneigung (maximal $40^{\circ}$ ) für die Errichtung von Freiflächen-Photovoltaikanlagen geeignet. Inwiefern diese Flächen z. B. aufgrund des Untergrundes oder der geplanten Nachnutzung (z. B. Rekultivierung) genutzt werden können, kann hier nicht beurteilt werden.

Wie viel Fläche aus militärischer Konversion in den Jahren bis 2030 frei wird, lässt sich nicht bestimmen. Derzeit werden fast 550.000 ha in Deutschland militärisch genutzt. Mehr als die Hälfte dieser Fläche steht jedoch unter Naturschutz (Naturschutzgebiet, Natura 2000-Gebiet). Werden davon noch die Flächen subtrahiert, die Wald, Wasserfläche 
oder Feuchtgebiet sind oder bereits für Wohnzwecke oder landwirtschaftlich genutzt werden, so bleiben noch etwas mehr als 54.000 ha übrig. Werden hiervon nur die Flächen berücksichtigt, die eine südliche Ausrichtung und eine geeignete Hangneigung aufweisen, beläuft sich das verbleibende Potenzial auf weniger als 20.000 ha. Wie viel davon in den nächsten Jahren aus der militärischen Nutzung genommen wird, ist unbekannt. Das theoretisch verfügbare Potenzial aus wirtschaftlichen und militärischen Konversionsflächen beläuft sich demnach auf etwa 26.000 ha. Würden ausschließlich diese Flächen genutzt, könnten die heute von Freiflächen-Photovoltaikanlagen in Anspruch genommene Fläche mehr als verdoppelt werden, ohne weitere Landwirtschaftsfläche in Anspruch zu nehmen oder die Siedlungsentwicklung zu beeinträchtigen. Ob dies die Flächennachfrage decken würde, kann hier nicht beantwortet werden.

Bei dieser Analyse handelt es sich ausschließlich um eine GIS-gestützte Analyse verfügbarer Datensätze und erhebt keinen Anspruch auf Korrektheit der verfügbaren Potenziale von erneuerbaren Energien auf für Siedlungszwecke ungeeigneten nichtagrarischen Flächen.

\subsubsection{Strategien „Siedlung und Verkehr"}

Die im Sektor Siedlung- und Verkehr modellierten Maßnahmen und deren Zuordnung zu den Strategien, die intensiv mit nationalen und regionalen Akteuren der Landnutzung diskutiert wurden, sind in Tab. 4.7 aufgeführt. Tab. 4.8 bündelt die Flächennutzung für Siedlung und Verkehr sowie Landwirtschaft und Wald in der Referenzprojektion sowie den untersuchten Landnutzungsstrategien.

\subsubsection{Ergebnisse der Klimaschutzstrategie}

Für eine klimaschützende Landnutzungsstrategie werden im Sektor Siedlung und Verkehr Maßnahmen gebündelt, die eine ressourcenschonende Siedlungsentwicklung und effiziente Siedlungsstrukturen fördern. Mit Blick auf die Relevanz für die Flächennutzung geht es in erster Linie um die Reduzierung der Flächeninanspruchnahme, wodurch Kohlenstoffsenken erhalten bleiben. Zudem ermöglicht gezielte Innenentwicklung die effizientere Ausnutzung von Infrastruktur. Laut Jenssen (2009, S. 283) könne „ein wichtiges Element zur THG-Minderung [...] die Gestaltung der zukünftigen Siedlungsentwicklung durch dichte Siedlungsformen sein“, was durch entsprechende Studien belegt ist (Dodman 2009; Hamin und Gurren 2009; Jenssen 2009). Es geht in der Klimaschutzstrategie genauso wenig wie in den auf die Erhöhung der städtebaulichen Dichte ausgerichteten Einzelmaßnahmen um ,bedingungslose Dichte im Namen des Klimaschutzes“ (Jenssen 2009, S. 283), sondern um eine Dichte, die mit hoher städtebaulicher Qualität einhergeht. 
Tab.4.7 Maßnahmen zur Steuerung der Siedlungs- und Verkehrsflächenentwicklung und Zuordnung der Maßnahmen zu Strategien. (Quelle: J. Hoymann und R. Goetzke)

\begin{tabular}{|c|c|c|c|c|}
\hline Maßnahme & Klimaschutz & Bioenergie* & $\begin{array}{l}\text { Natur- und } \\
\text { Umweltschutz }\end{array}$ & $\begin{array}{l}\text { Klima- } \\
\text { anpassung }\end{array}$ \\
\hline Stärkung der Innenentwicklung & +++ & $(+)$ & ++ & $+1-$ \\
\hline $\begin{array}{l}\text { Ausschöpfung baulicher Dichte } \\
\text { im Neubau }\end{array}$ & ++ & $(+)$ & + & $+1-$ \\
\hline $\begin{array}{l}\text { Reduktion der Flächeninan- } \\
\text { spruchnahme durch Verkehr }\end{array}$ & ++ & $(+)$ & ++ & + \\
\hline $\begin{array}{l}\text { Rückzug aus der Fläche } \\
\text { (dezentrale Konzentration) }\end{array}$ & $++/-$ & $(+)$ & ++ & ++ \\
\hline $\begin{array}{l}\text { Erhalt und Entwicklung inner- } \\
\text { städtischer Freiflächen }\end{array}$ & + & $(++)$ & + & +++ \\
\hline Stärkung des ÖPNV & +++ & $\mathrm{O}$ & + & $\mathrm{O}$ \\
\hline $\begin{array}{l}\text { Zusätzliche Ausweisung von Vor- } \\
\text { rang- und Vorbehaltsgebieten }\end{array}$ & ++ & $(+)$ & ++ & ++ \\
\hline $\begin{array}{l}\text { Stärkung des Hochwasser- } \\
\text { schutzes }\end{array}$ & $\mathrm{O}$ & $\mathrm{O}$ & $\mathrm{O}$ & +++ \\
\hline Restriktiverer Freiraumschutz & + & $\mathrm{O}$ & +++ & $\mathrm{O}$ \\
\hline $\begin{array}{l}\text { Energieerzeugung auf für } \\
\text { Siedlungszwecke ungeeigneten } \\
\text { nicht-agrarischen Flächen }\end{array}$ & ++ & $(+)$ & $\mathrm{O}$ & $\mathrm{O}$ \\
\hline $\begin{array}{l}\text { Nutzung von Biomasse der Land- } \\
\text { schaftspflege }\end{array}$ & ++ & ++ & $\mathrm{O}$ & $\mathrm{O}$ \\
\hline
\end{tabular}

Anmerkung: Eignung der Maßnahme zur Erreichung des Ziels: $+=$ unter bestimmten Voraussetzungen geeignet, bzw. Teile der Maßnahme geeignet, $++=$ gut geeignet, $+++=$ sehr gut geeignet, $+(++) /-=$ geeignet, aber unter Umständen gegenläufige Wirkung, $\mathrm{O}=$ keine Wirkung *Die Strategie „Bioenergie“ wurde im Sektor Siedlung und Verkehr nur am Rande untersucht, weil damit keine flächenrelevanten Landmanagementmaßnahmen aus Sicht der Siedlungs- und Verkehrsflächenentwicklung verbunden sind. Allerdings können einzelne Bestandteile anderer Maßnahmen einen Beitrag zur Strategie „Bioenergie“ leisten, beispielsweise dadurch, dass sie durch die Verringerung des Siedlungsdrucks auf die Landwirtschaftsfläche die dortigen Nutzungskonkurrenzen entschärfen. Diese Maßnahmen sind mit () gekennzeichnet

Insbesondere im Zusammenspiel mit Maßnahmen zur Reduzierung des Verkehrsaufkommens können hierdurch erhebliche $\mathrm{CO}_{2}$-Emissionen eingespart werden. Die $\mathrm{CO}_{2}$ Emissionen des Verkehrssektors beliefen sich im Jahr 2006 auf 160 Mio. t, wovon $93 \%$ auf den Straßenverkehr entfallen (infas Institut für angewandte Sozialwissenschaften GmbH und DLR Institut für Verkehrsforschung 2010, S. 1). Damit ist der Straßenverkehr für knapp $16 \%$ der Gesamt-Emissionen an $\mathrm{CO}_{2}$ verantwortlich (Umweltbundesamt 2013, S. 196). Würde beispielsweise das Verhältnis von ÖPNV zu MIV von derzeit 1:5 auf 2:1 verändert werden, hätte dies eine beachtliche Wirkung auf die gesamte THG-Bilanz 
Tab. 4.8 Flächennutzung im jahr 2030 für Siedlung und Verkehr sowie Landwirtschaft und Wald in der Referenzprojektion sowie den untersuchten Landnutzungsstrategien. (Quelle: R. Goetzke)

\begin{tabular}{|c|c|c|c|c|c|c|c|c|c|}
\hline & \multirow{2}{*}{ 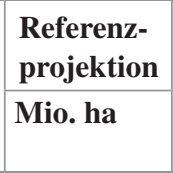 } & \multicolumn{2}{|c|}{ Klimaschutz } & \multicolumn{2}{|c|}{ Biomasse } & \multicolumn{2}{|c|}{$\begin{array}{l}\text { Natur- und } \\
\text { Umweltschutz }\end{array}$} & \multicolumn{2}{|c|}{$\begin{array}{l}\text { Klima- } \\
\text { anpassung }\end{array}$} \\
\hline & & Mio. ha & $\begin{array}{l}\% \text { vs. } \\
\text { Ref. }\end{array}$ & Mio. ha & $\begin{array}{l}\text { \% vs. } \\
\text { Ref. }\end{array}$ & Mio. ha & $\begin{array}{l}\text { \% vs. } \\
\text { Ref. }\end{array}$ & Mio. ha & $\begin{array}{l}\text { \% vs. } \\
\text { Ref. }\end{array}$ \\
\hline $\begin{array}{l}\text { Siedlungs- } \\
\text { und Ver- } \\
\text { kehrsfläche }\end{array}$ & 5,133 & 4,915 & $-4,2$ & 4,977 & $-3,0$ & 4,957 & $-3,4$ & 5,081 & $-1,0$ \\
\hline $\begin{array}{l}\text { davon } \\
\text { Gebäude } \\
\text { und Frei- } \\
\text { fläche }\end{array}$ & 2,630 & 2,463 & $-6,3$ & 2,516 & $-4,3$ & 2,499 & $-5,0$ & 2,583 & $-1,8$ \\
\hline $\begin{array}{l}\text { davon } \\
\text { Erholungs- } \\
\text { fläche }\end{array}$ & 0,498 & 0,481 & $-3,2$ & 0,484 & $-2,7$ & 0,484 & $-2,8$ & 0,517 & 4,0 \\
\hline $\begin{array}{l}\text { davon Ver- } \\
\text { kehrsfläche }\end{array}$ & 1,914 & 1,875 & $-2,0$ & 1,881 & $-1,7$ & 1,879 & $-1,8$ & 1,886 & $-1,5$ \\
\hline $\begin{array}{l}\text { davon } \\
\text { Betriebs- } \\
\text { fläche ohne } \\
\text { Abbauland }\end{array}$ & 0,095 & 0,095 & 0,0 & 0,095 & 0,0 & 0,095 & 0,0 & 0,095 & 0,0 \\
\hline $\begin{array}{l}\text { Landwirt- } \\
\text { schafts- } \\
\text { fläche }\end{array}$ & 18,019 & 17,444 & $-3,2$ & 18,174 & 0,9 & 18,194 & 1,0 & 18,174 & 0,9 \\
\hline Waldfläche & 11,056 & 11,848 & 7,2 & 11,056 & 0,0 & 11,056 & 0,0 & 11,056 & 0,0 \\
\hline
\end{tabular}

(Fuhrich et al. 2004; infas Institut für angewandte Sozialwissenschaften GmbH und DLR Institut für Verkehrsforschung 2010). Gezielte Maßnahmen können dies befördern, wie die Konzentration der Siedlungsentwicklung auf zentrale Orte und effiziente (ÖPNV-) Verkehrsknotenpunkte oder die Reduzierung der Verkehrsflächenentwicklung sowie eine insgesamt verdichtete Siedlungsentwicklung. Die Abschätzung der dadurch möglichen THG-Einsparung ist nicht Gegenstand dieser Studie (vgl. Kap. 1).

Die Strategie „Klimaschutz“ wird durch die Kombination folgender Maßnahmen modelliert:

- Stärkung der Innenentwicklung

- Ausschöpfung baulicher Dichte im Neubau

- Stärkung des ÖPNV

- Reduktion der Flächeninanspruchnahme durch Verkehr

- Rückzug aus der Fläche 
- Restriktiverer Freiraumschutz

- Zusätzliche Ausweisung von Vorrang- und Vorbehaltsgebieten (nur solche, die einen Klimaschutzbezug haben, z. B. durch Freiraumschutz, Bodenschutz oder Förderung erneuerbarer Energien)

- Energieerzeugung auf für Siedlungszwecke ungeeigneten nicht-agrarischen Flächen

Darüber hinaus fließt aus der Maßnahme „Erhalt und Entwicklung innerstädtischer Freiflächen“ das Element „Rückbau, Entsiegelung und Konzentration gebauter Strukturen“ in die Strategie ein. Dabei gilt die Annahme, dass entsiegelte Flächen innerhalb des Siedlungskörpers begrünt werden und somit einen Beitrag zur Kohlenstoffspeicherung leisten können.

Neben den Maßnahmen aus dem Siedlungssektor, die zu dieser Strategie beitragen, wird zusätzlich eine sektorübergreifende Maßnahme „Aufforstung“ betrachtet. In dieser Maßnahme wird von einer Erhöhung der Waldfläche um $10 \%$ oder $10.800 \mathrm{~km}^{2}$ ausgegangen, wodurch der Waldflächenanteil von 30 auf $33 \%$ der Bundesfläche steigen würde. In den anderen Landnutzungsstrategien wird ein kontinuierlicher Flächenzuwachs des Waldes von insgesamt knapp $3 \%$ angenommen, wodurch sich die Waldfläche bis 2030 von 30 auf $31 \%$ erhöhen würde.

Aufgrund der Kombination mehrerer Maßnahmen, die auf die Reduzierung der Flächeninanspruchnahme abzielen, ergibt sich in der Klimaschutzstrategie laut Modellrechnung eine deutliche Reduktion der täglichen Flächeninanspruchnahme auf etwa 20 ha im Zeitraum 2026 bis 2030 (vgl. Abb. 4.24). Insgesamt würden zwischen 2010 und 2030 fast $2200 \mathrm{~km}^{2}$ weniger Fläche für Siedlungs- und Verkehrszwecke in Anspruch genommen, als in der Referenzprojektion. Das entspräche etwa der 2,5-fachen Fläche Berlins. Der Rückgang der Flächenneuinanspruchnahme wird vom Rückgang der Gebäude- und Freifläche getragen, auf die die Maßnahmen insbesondere abzielen. Ein leichter Rückgang der Verkehrsflächenentwicklung gegenüber der Referenzprojektion ergibt sich aus der geringeren Anzahl benötigter Erschließungsstraßen. Auch die Erholungsflächenentwicklung wäre rückläufig, da weniger Ausgleichs- und Ersatzmaßnahmen umgesetzt werden müssten. Ab etwa 2025 würde netto keine zusätzliche Gebäude- und Freiflächenentwicklung mehr stattfinden. Sie könnte durch den fortlaufenden Rückbau von Leerständen und die damit einhergehende Konzentration auf bereits bebaute Strukturen unter Null sinken.

Auch wenn deutschlandweit betrachtet in der Klimaschutzstrategie die Flächeninanspruchnahme deutlich reduziert werden kann, ist davon auszugehen, dass es weiterhin Regionen mit hohem Flächenzuwachs gibt (vgl. Abb. 4.25). Von einem weiterhin hohen Flächenzuwachs trotz der durchgeführten Maßnahmen wird im Umland der großen Großstädte (v. a. Hamburg, Berlin, München), im Rheinland, der Metropolregion Rhein-Neckar und im westlichen Niedersachsen (Emsland) auszugehen sein. In weiten Landesteilen Sachsens, Sachsen-Anhalts und Mecklenburgs könnte aufgrund des hohen 


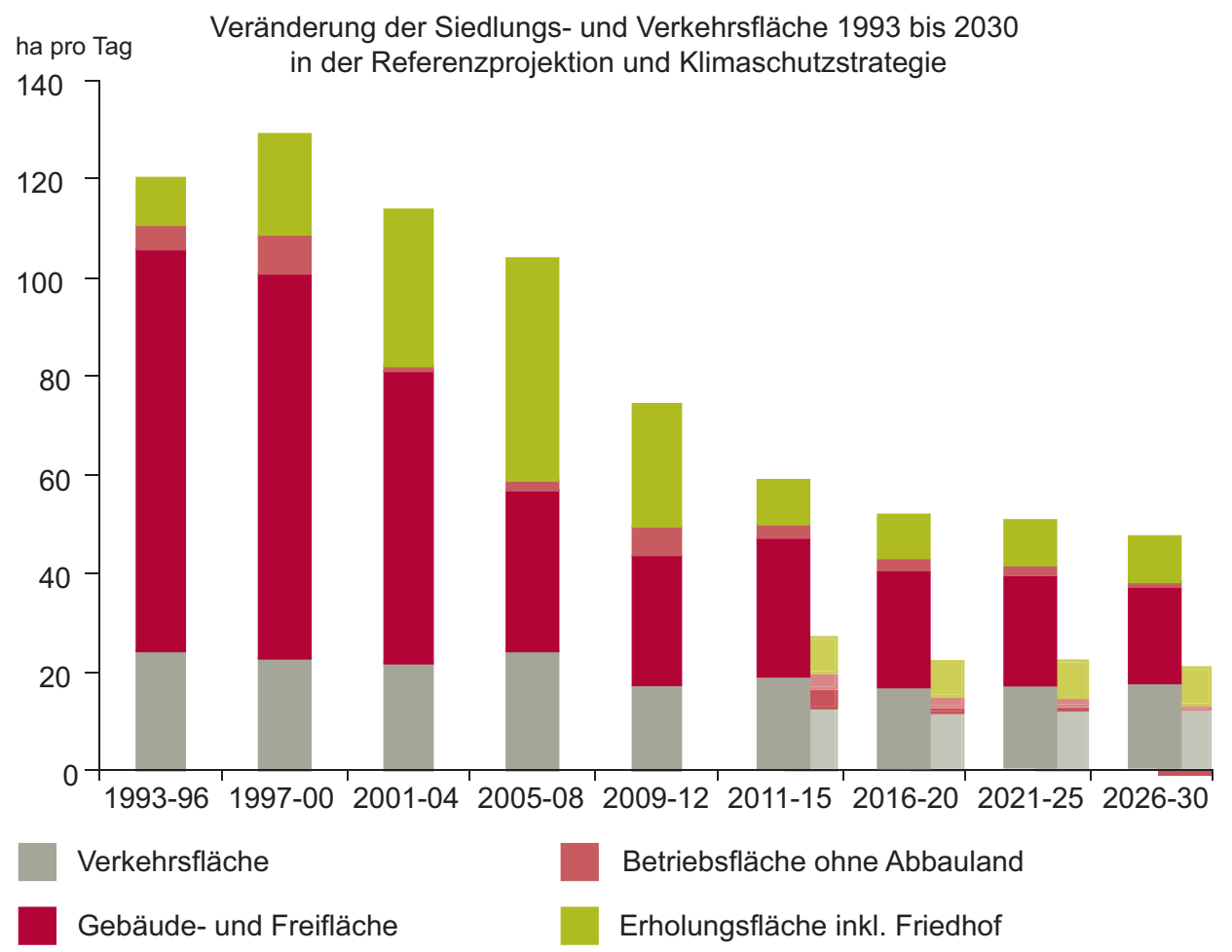

Abb. 4.24 Veränderung der Siedlungs- und Verkehrsfläche 1993 bis 2030 in der Referenzprojektion und Klimaschutzstrategie. (Quelle: Flächenerhebung des Bundes und der Länder, GWS Osnabrück 2012, Berechnungen des BBSR)

Leerstands ein Netto-Rückbau stattfinden, der entsprechend begleitet werden muss, um die bestehenden Strukturen zu stärken. In vielen kreisfreien Städten könnte die Baulandnachfrage vollständig durch Innenentwicklung befriedigt werden. Hierzu zählen viele Städte im Ruhrgebiet (z. B. Essen, Dortmund, Herne), von denen einige aufgrund des hohen Leerstands auch Netto-Rückbau betreffen könnte (z. B. Gelsenkirchen, Bochum). Aber auch andere kreisfreie Städte - vor allem solche mit Bevölkerungsrückgang könnten ihre Außenentwicklung durch Maßnahmen der Innenentwicklung, Konzentration und Entsiegelung drastisch reduzieren, wie z. B. Offenbach, Ludwigshafen, Wuppertal, Solingen, Krefeld oder Kassel.

In der Klimaschutzstrategie wird neben den Annahmen zu Maßnahmen im Siedlungsund Verkehrsbereich ein Aufforstungsszenario berücksichtigt, bei dem die Waldfläche bis 2030 um $10 \%$ zunimmt. Dies wirkt sich lokal auf die räumliche Verteilung der Siedlungsflächenentwicklung aus, da durch die zusätzlichen Flächenansprüche manche Flächen eine gewisse Eignung sowohl für die Ausweitung von Siedlungsgebieten aufweisen als auch für Aufforstung. Insgesamt ändert sich das Muster der 


\section{Entwicklung der Siedlungs- und Verkehrsfläche in der Referenzprojektion und in den Strategien zwischen 2010 und 2030 in den Gemeinden}
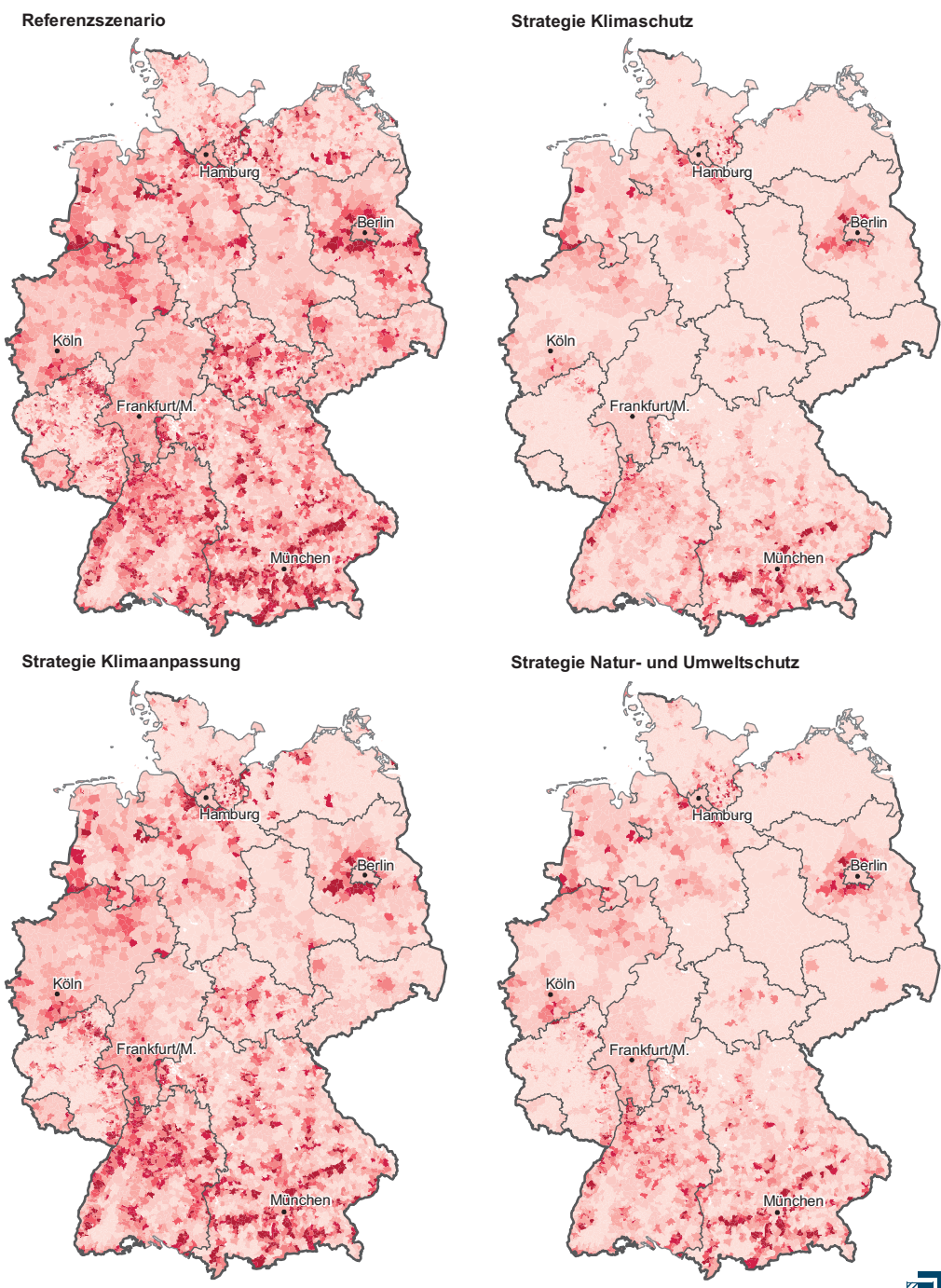

$100 \mathrm{~km}$

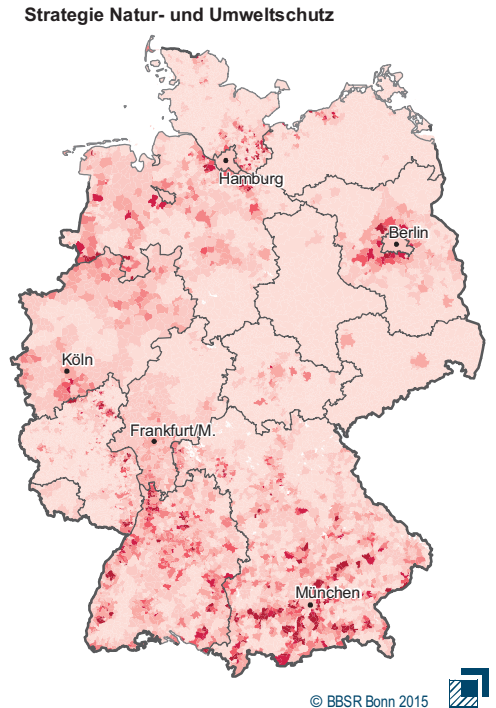

Veränderung der Siedlungs- und Verkehrsfläche zwischen 2010 und 2030 in \%

$$
\begin{aligned}
& \quad 0 \text { und weniger } \\
& >0 \text { bis unter } 5 \\
& 5 \text { bis unter } 10 \\
& 10 \text { bis unter } 15 \\
& 15 \text { bis unter } 20 \\
& 20 \text { bis unter } 25 \\
& 25 \text { und mehr }
\end{aligned}
$$

Quelle: Laufende Raumbeobachtung des BBSR, GWS Osnabrück 2012, Berechnungen des BBSR

Geometrische Basis: BKG/BBSR, Gemeinden 31.12.2011

Bearbeitung: J. Hoymann, R. Goetzke

Abb. 4.25 Entwicklung der Siedlungs- und Verkehrsfläche in der Referenzprojektion und in den Strategien zwischen 2010 und 2030 in den Gemeinden. Referenzprojektion (oben links), Klimaschutzstrategie (oben rechts), Klimaanpassungsstrategie (unten links), Natur- und Umweltschutzstrategie (unten rechts) 
Siedlungsentwicklung jedoch nicht, da durch die getroffenen Annahmen zur Aufforstung Waldmehrung vor allem in Gebieten mit einem hohen Anteil an Grenzertragsstandorten stattfindet. Historisch gesehen sind dies nicht die Gegenden, in denen größere Siedlungsgebiete entstanden sind, sondern in weiten Teilen periphere ländliche Regionen. Die höchsten absoluten Zuwächse der Waldfläche finden sich unter dieser Annahme beispielsweise in Brandenburg (z. B. Uckermark, Havelland, Potsdam-Mittelmark), Sachsen-Anhalt (Altmark, Börde), im Alpenvorland (z. B. Weilheim-Schongau, Bad Tölz-Wolfrathshausen, Oberallgäu) und auf der Schwäbischen Alb (z. B. Zollernalbkreis, Reutlingen).

Um die Wirkung der in der Klimaschutzstrategie verwendeten Maßnahmen zu bewerten, werden unterschiedliche Indikatoren herangezogen, wie die „Verkehrliche Erschließung neuer Siedlungsflächen“. Während in der Referenzprojektion neue Siedlungsflächen im Durchschnitt mit einer Fahrtzeit von 28 min per öffentlichem Verkehr vom nächsten Mittel- oder Oberzentrum entstehen, sind es in der Klimaschutzstrategie im Schnitt 25 min (vgl. zur Erläuterung Abschn. 4.4.2.5). ${ }^{12}$ Die Maßnahmen der Klimaschutzstrategie führen demnach dazu, dass nicht nur weniger neue Siedlungsflächen benötigt werden, sondern auch zu einer besseren verkehrlichen Erreichbarkeit. Dies unterstützt eine Veränderung des Modal Split und kann den hohen Anteil des MIV und damit beträchtliche THG-Emissionen reduzieren.

Die insgesamt kompaktere Siedlungsentwicklung (Leitbild ist die Stadt der kurzen Wege) lässt sich mit Hilfe des Indikators „Standörtliche Integration neuer Siedlungsflächen“ messen. Zur Berechnung dieses Indikators wird die gemeinsame Randlänge neuer und bestehender Siedlungs- und Verkehrsflächen durch den Umfang neuer Siedlungs- und Verkehrsflächen geteilt. Dies erlaubt Aussagen über die räumliche Konzentration der Siedlungsentwicklung, bzw. über das Ausmaß der Zersiedelung (BMVBS 2007). Abb. 4.26 lässt erkennen, dass durch das Maßnahmenbündel der Klimaschutzstrategie in den meisten Regionen eine bessere Integration neuer Siedlungsflächen in bestehende Siedlungskörper erreicht werden kann. Dies ist in $86 \%$ der Kreise der Fall. Die standörtliche Integration neuer Siedlungsflächen verbessert sich vor allem in den Regionen, die in der Klimaschutzstrategie kaum noch nennenswerte Flächenerweiterungen aufweisen (z. B. Altmark). In diesen Regionen sind die wenigen neu hinzukommenden Siedlungsflächen gut in den Siedlungsbestand integriert, woraus sich eine deutliche Verbesserung des Indexwertes ergibt (vgl. Abschn. 4.4.2.2).

\footnotetext{
${ }^{12}$ Die durchschnittliche Fahrtzeit liegt etwas höher als im Maßnahmen-Szenario „Stärkung des ÖPNV“, da in der Klimaschutzstrategie noch andere „konkurrierende“ Maßnahmen umgesetzt werden, die ihrerseits eine andere Zielrichtung haben, als die Siedlungsentwicklung möglichst verkehrsoptimiert zu steuern (z. B. Maßnahme „Zusätzliche Ausweisung von Vorrang- und Vorbehaltsgebieten").
} 


\section{Integration neuer Siedlungsflächen in den Siedlungsbestand durch Maßnahmen der Klimaschutzstrategie}

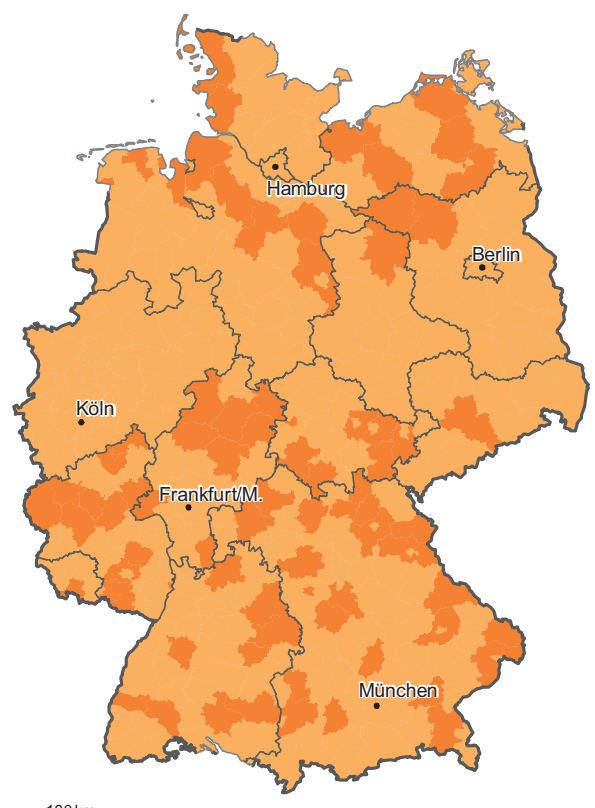

$100 \mathrm{~km}$

\section{Klimaschutzstrategie}

(Abweichung vom Bundesdurchschnitt)

$$
\begin{aligned}
& \text { deutlich bessere standörtliche Integration } \\
& \text { leicht bessere standörtliche Integration } \\
& \text { leicht schlechtere standörtliche Integration } \\
& \text { deutlich schlechtere standörtliche Integration }
\end{aligned}
$$

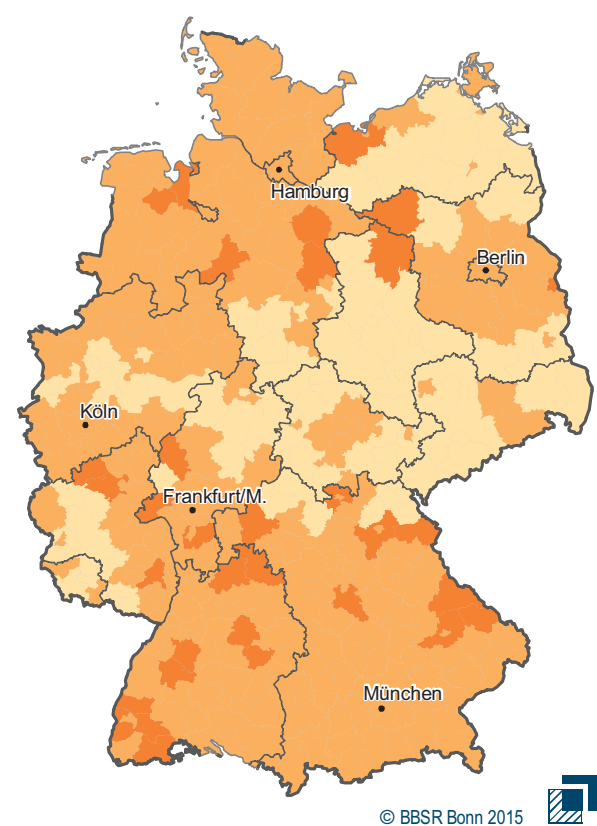

Datenbasis: Laufende Raumbeobachtung des BBSR, Berechnungen des BBSR

Geometrische Datenbasis: BKG/BBSR Kreise31.12.2013

Bearbeitung: J. Hoymann, R. Goetzke

Abb. 4.26 Standörtliche Integration neuer Siedlungsflächen als Abweichung vom Bundesdurchschnitt in der Referenzprojektion (links) und in der Klimaschutzstrategie (rechts)

Durch die Klimaschutzmaßnahmen im Siedlungs- und Verkehrssektor ergeben sich auch Änderungen in der Kohlenstoffbilanz der betroffenen Landnutzung. Auf versiegelten Flächen findet weder Sequestrierung noch Emission von Kohlenstoff durch den Boden statt. Je nach Humusgehalt des Oberbodens und Bewirtschaftungsweise wird auf Ackerflächen weniger Kohlenstoff eingespart als auf Grünland oder im Wald oder sogar netto emittiert. Abhängig davon, auf welchen Flächen sich die Siedlungs- und Verkehrsfläche ausdehnt und wie diese zusammengesetzt ist (Grünflächen, Versiegelungsgrad), ergeben sich Veränderungen in der Kapazität von Boden und Vegetation zur Bindung von Kohlenstoff. Demnach wirkt eine klimaschützende Siedlungsentwicklung vor allem dort, wo Flächeninanspruchnahme hauptsächlich auf Grünland stattfindet. 


\subsubsection{Ergebnisse der Bioenergiestrategie}

Im Siedlungs- und Verkehrsbereich werden keine Maßnahmen der Bioenergiestrategie modelliert, da keine flächenrelevanten Maßnahmen identifiziert wurden. Jedoch können Maßnahmen, die nicht primär auf die Förderung von Biomasse abzielen, eine Bioenergiestrategie trotzdem unterstützen. So können städtische Grünflächen erhalten und ausgeweitet werden (Maßnahme „Erhalt und Entwicklung innerstädtischer Freiflächen“), sodass der dort entstehende Grünschnitt energetisch weiterverarbeitet werden kann (vgl. Abschn. 4.4.2.4). Des Weiteren trägt eine Reduzierung der Flächenneuinanspruchnahme durch Siedlung und Verkehr zu verminderten Flächenkonkurrenzen auf der Landwirtschaftsfläche bei, was ein Nebeneinander von Pflanzen zur energetischen Nutzung und zur Nahrungs- und Futtermittelnutzung erleichtert. Es wurde festgestellt, dass alle Maßnahmen im Siedlungs- und Verkehrsbereich aus der Natur- und Umweltschutzstrategie auch einen positiven Effekt auf die Bioenergiestrategie haben. $\mathrm{Zu}$ welchem Zweck die von Siedlungsentwicklung freigehaltenen Flächen genutzt werden (Biomasse oder Naturschutz), ist aus Sicht der Siedlungsflächenmodellierung unerheblich. Dies wird in den land- und forstwirtschaftlichen Modellen analysiert. Die für land- und forstwirtschaftliche Zwecke verbleibenden Flächensummen sind in den Strategien „Bioenergie“ und „Natur- und Umweltschutz“ nahezu identisch.

\subsubsection{Ergebnisse der Natur- und Umweltschutzstrategie}

In der Natur- und Umweltschutzstrategie werden Maßnahmen durchgeführt, die eine nachhaltige Landnutzung zum Ziel haben und dabei eine Siedlungs- und Verkehrsflächenentwicklung fördern, die verstärkt Natur- und Umweltschutzaspekte berücksichtigt. Der Klimawandel steht dabei nicht im Mittelpunkt des Interesses, doch können Natur- und Umweltschutzmaßnahmen durchaus positive Klimawirkungen entfalten. Andersherum betrachtet können Klimaschutz- und Klimaanpassungsaspekte zusätzliche Argumente für die Durchführung von Natur- und Umweltschutzmaßnahmen im Siedlungs- und Verkehrsbereich sein. Das Zusammenwirken von Stadtnaturschutz und Gesundheitsaspekten wird von Rittel et al. (2014) ausführlich beschrieben. Beim Thema Brachflächen gibt es nicht nur teils divergierende Interessen in Bezug auf Klimaschutz (Nachverdichtung) und -anpassung (Freiraum), hinzu kommen auch Naturschutzinteressen, weil innerstädtische Brachflächen oft bedeutende Standorte für seltene Arten darstellen und damit eine wichtige Rolle für die biologische Vielfalt in der Stadt spielen (Hansen et al. 2012). Hier gilt es, einen Ausgleich zwischen Erholungsnutzung, Förderung der Biodiversität und berechtigten Nachverdichtungsinteressen herzustellen.

Naturschutzpolitische Instrumente können auch einen Beitrag zur Steuerung der Flächeninanspruchnahme leisten, wie Heiland et al. (2006) ausführlich darstellen. Dies kann wiederum Klimaschutzinteressen positiv unterstützen. Einige dieser Instrumente, wie beispielsweise Landschaftsschutzgebiete, werden bislang kaum zur Steuerung der Siedlungsflächenentwicklung in Betracht gezogen. Mit Hilfe der Landnutzungsmodellierung kann dargestellt und analysiert werden, welche Wirkung sich aus einer 
Stärkung naturschutzpolitischer Instrumente in Bezug auf die Siedlungsentwicklung ergeben kann.

In der Modellierung werden Maßnahmen kombiniert, die sich in weiten Teilen auch in den Strategien Klimaschutz bzw. Klimaanpassung wiederfinden. Folgende Maßnahmen werden in der Natur- und Umweltschutzstrategie modelliert:

- Stärkung der Innenentwicklung (allerdings geringere Wiedernutzung von Brachflächen als in der Klimaschutzstrategie)

- Ausschöpfung baulicher Dichte im Neubau

- Reduzierung der Flächeninanspruchnahme durch Verkehr

- Rückzug aus der Fläche/dezentrale Konzentration

- Erhalt und Entwicklung innerstädtischer Freiflächen (Konzentration auf Erhalt innerstädtischer Grünflächen)

- Zusätzliche Ausweisung von Vorrang- und Vorbehaltsgebieten (nur solche mit Bezug zu Natur- und Umweltschutzzielen)

- Restriktiverer Freiraumschutz

In der Natur- und Umweltschutzstrategie liegt die Flächenneuinanspruchnahme durch Siedlung und Verkehr nach Modellrechnungen im Zeitraum 2026 bis 2030 bei etwa 25 ha/Tag. Dieser Rückgang ergibt sich vor allem aus einem deutlich geringeren Bedarf an Gebäude- und Freifläche mit 8 ha/Tag gegenüber 19 ha/Tag in der Referenzprojektion. Aber auch die Verkehrsflächenentwicklung ist mit unter 13 ha/Tag niedriger als in der Referenzprojektion mit 15 ha/Tag (vgl. Abb. 4.27). Die deutlichen Rückgänge der täglichen Flächeninanspruchnahme in der Natur- und Umweltschutzstrategie haben ein ähnliches räumliches Muster wie in der Klimaschutzstrategie, allerdings sind sie insgesamt weniger ausgeprägt.

In Bezug auf die Menge der Flächeninanspruchnahme ist die Natur- und Umweltschutzstrategie ein Mittelweg zwischen den Strategien Klimaschutz und Klimaanpassung, denn sie berücksichtigt sowohl Aspekte des Flächensparens als auch wertvolle Freiräume. Auch die beiden anderen Strategien gehen nicht an die Grenze ihrer Möglichkeiten bspw. hinsichtlich der Ausschöpfung von Innenentwicklungspotenzialen oder des Rückbaus, da die Maßnahmen innerhalb der Strategien immer im Bereich des städtebaulich sinnvollen bzw. machbaren bleiben. Doch in der Natur- und Umweltschutzstrategie finden sich Elemente von beiden „Polen“.

Hinsichtlich des räumlichen Musters der Siedlungsentwicklung ergeben sich in der Natur- und Umweltschutzstrategie spezifische Besonderheiten, die beispielsweise aus der Stärkung von Schutzgebieten, z. B. durch Pufferung, resultieren. Insgesamt verortet das Landnutzungsmodell nur noch ein Drittel der in der Referenzprojektion in Schutzgebieten verorteten Siedlungsflächen in diesen Gebieten. An dieser Stelle sei angemerkt, dass es in der Klimaschutzstrategie noch weniger sind, da die Flächenneuinanspruchnahme insgesamt geringer ausfällt. Aus dieser Erkenntnis ergeben sich aus rein quantitativer 


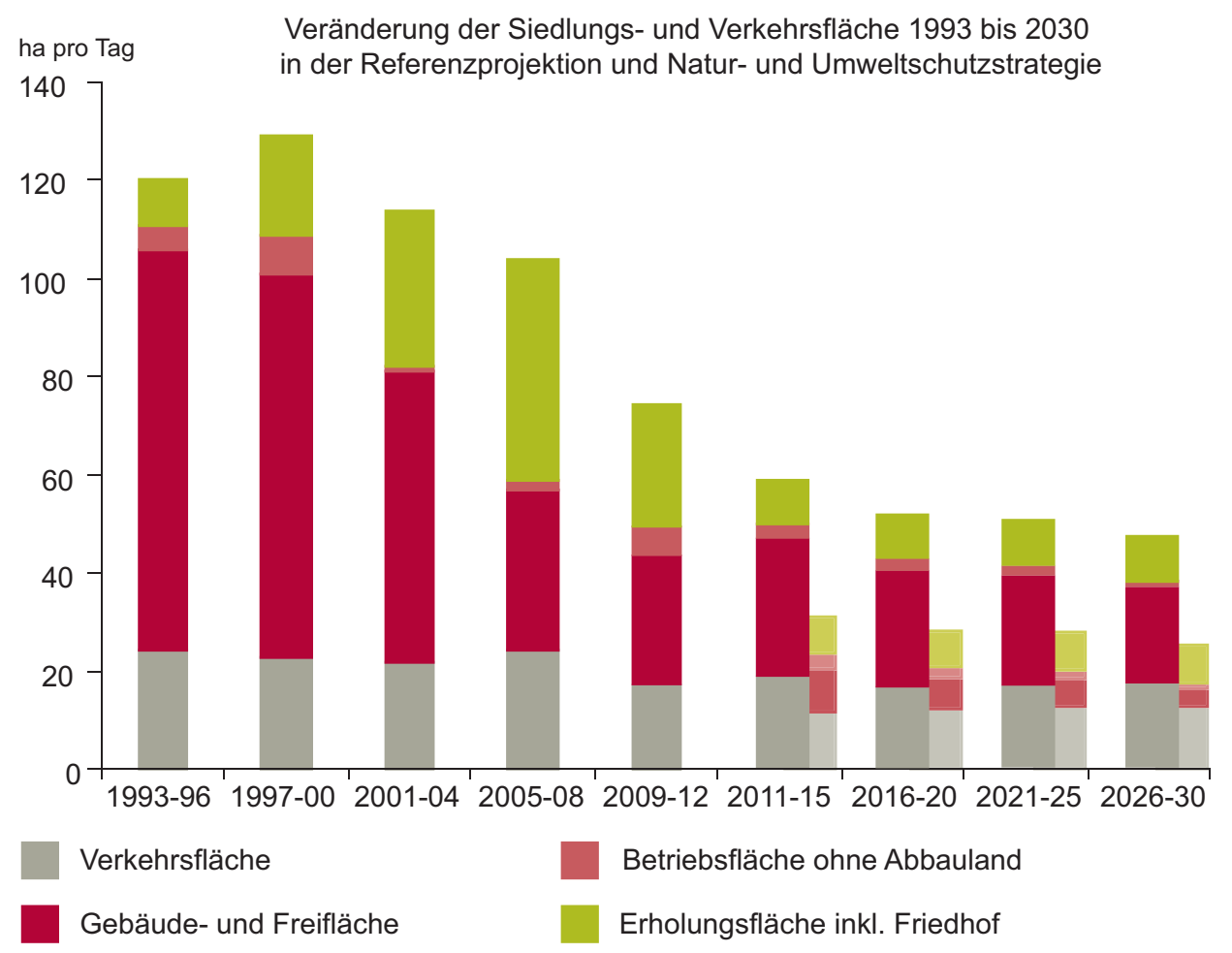

Abb. 4.27 Veränderung der Siedlungs- und Verkehrsfläche 1993 bis 2030 in der Referenzprojektion und Natur- und Umweltschutzstrategie

Sicht Argumentationsmöglichkeiten, dass Klimaschutz dem Naturschutz, bzw. Naturschutz dem Klimaschutz dienlich sein kann. Hierfür bedarf es allerdings einer genaueren Betrachtung, was auf den entsprechenden Flächen genau passiert.

Deutlich ist in der Natur- und Umweltschutzstrategie der Rückgang der Siedlungsund Verkehrsflächenentwicklung in unzerschnittenen Räumen. Während in der Referenzprojektion das Modell noch mehr als 15.000 ha bis 2030 in diesen Räumen verortet, sind es in der Natur- und Umweltschutzstrategie nur noch weniger als 4000 ha (in der Klimaschutzstrategie 3000 ha).

Somit lässt sich festhalten, dass in der Klimaschutzstrategie zwar noch mehr schützenswerte Räume erhalten bleiben als in der Natur- und Umweltschutzstrategie, diese aber die Möglichkeiten der Kommunen bei der Ausweisung von Baugebieten deutlich beschränkt. Die Maßnahmen der Natur- und Umweltschutzstrategie sind hinsichtlich des Flächensparens weniger restriktiv, bewirken aber dennoch eine erkennbare Verbesserung beim Erhalt von aus naturschutzfachlicher Sicht schützenswerter Räume. 


\subsubsection{Ergebnisse der Klimaanpassungsstrategie}

Im Siedlungs- und Verkehrsbereich sind, ähnlich wie im Forst, Maßnahmen zur Anpassung an den Klimawandel von wesentlich größerer Bedeutung als in der Landwirtschaft, mit denen kurzfristiger auf sich ändernde Umweltbedingungen reagiert werden kann. Heutige Planungen von Baugebieten und Verkehrsinfrastruktur manifestieren Landnutzungsänderungen auf Jahrzehnte hinaus und sind nur unter hohem Kostenaufwand wieder zu verändern. Aus den prognostizierten Auswirkungen des Klimawandels in Deutschland, wie lang anhaltende Hitzewellen im Sommer, zunehmende Starkregen und häufiger wiederkehrende extreme Hochwasserereignisse, ergeben sich besondere Anforderungen an den Siedlungs- und Infrastrukturbau zum Schutz von Menschen und Sachgütern (vgl. Bundesregierung 2008, S. 19 f.).

Anpassung kann im Bereich des Bauwesens geschehen, beispielsweise durch hochwasserangepasste Bauweise, Ausbau der Kanalisation, hitzebeständigere Materialien im Straßenbau oder durch Fassaden mit hoher Albedo. Im Fokus stehen hier hingegen flächenrelevante Maßnahmen. Eine besondere Bedeutung kommt in diesem Zusammenhang der Raum-, Regional- und Bauleitplanung zu, wie schon die Deutsche Anpassungsstrategie an den Klimawandel feststellt (Bundesregierung 2008, S. 42): „Die Raum-, Regional- und Bauleitplanung stehen am Anfang der Risikovermeidungskette, da sie räumliche Vorsorgekonzepte entwickeln, die Planungsdokumente hohe Bestandsdauer und rechtliche Verbindlichkeit besitzen und bis zur praktischen Umsetzung der Planinhalte teilweise lange Vorlaufzeiten entstehen. Der räumlichen Planung kommt dabei die wichtige Aufgabe zu, verschiedene Ansprüche an den Raum miteinander zu vereinbaren." Insbesondere die Raumordnung ist geeignet, entsprechende Risikovorsorge durch die Ausweisung von Vorrang- und Vorbehaltsgebieten und die Steuerung der Siedlungsentwicklung zu leisten (vgl. Spiekermann und Franck 2014). Dies geschieht im Bereich der Hochwasservorsorge - derzeit im Zusammenhang mit der Umsetzung der Hochwasserrisikomanagement-Richtlinie des europäischen Parlaments und des Rates (Richtlinie 2007/60/EG) - , aber auch bei der thermischen Entlastung von Städten kann die Raumordnung einen wichtigen Beitrag leisten, beispielsweise durch das Freihalten von Luftaustauschkorridoren. Zur Reduzierung des Hitzeinseleffekts (vgl. Oke 1997) müssen, wie auch bei anderen Anpassungsaspekten, die verschiedenen Planungsebenen ineinandergreifen, beispielsweise durch (Lülf 2008):

- „die Auflockerung der Bebauung,

- die Schaffung, Sicherung und klimaeffiziente Bewirtschaftung klimarelevanter Grünflächen sowie

- die Erhaltung, strukturelle Verbesserung und Schaffung von Luftleitbahnen (insbesondere linearer Grün- und Wasserflächen)“

Für konkrete Umsetzungsbeispiele aus der Praxis im Bereich Überflutungs- und Hitzevorsorge durch die Stadtentwicklung sei auf die Ergebnisse des Projektes „Strategie 
Klimaanpassung zur Überflutungsvorsorge verschiedener Siedlungstypen als kommunale Gemeinschaftsaufgabe“ aus dem Forschungsprogramm „Experimenteller Wohnungsund Städtebau" (ExWoSt) des Bundesministeriums für Umwelt, Naturschutz, Bau und Reaktorsicherheit (BMUB) verwiesen (BBSR 2015a, b).

Zur Modellierung der Klimaanpassungstrategie im Sektor Siedlung und Verkehr werden folgende Maßnahmen miteinander kombiniert:

- Erhalt und Entwicklung innerstädtischer Freiflächen

- Reduktion der Flächeninanspruchnahme durch Verkehr

- Rückzug aus der Fläche/dezentrale Konzentration

- Zusätzliche Ausweisung von Vorrang- und Vorbehaltsgebieten (nur solche mit Bezug zur Klimaanpassungstrategie, bspw. Freihalten von hochwasser- und lawinengefährdeten Bereichen, Stärkung des Netzes regionaler Grünzüge)

- Stärkung des Hochwasserschutzes

- Stärkung der Innenentwicklung (nur solche Maßnahmen, die keine weitere Verdichtung/Versiegelung nach sich ziehen, wie bspw. Leerstandswiedernutzung)

In der Klimaanpassungstrategie fällt die Reduzierung der Flächeninanspruchnahme geringer aus als in der Klimaschutzstrategie. Laut Modellrechnungen liegt der tägliche Zuwachs an Siedlungs- und Verkehrsfläche im Zeitraum 2026-2030 bei etwas unter 40 ha (vgl. Abb. 4.28). Der im Vergleich zur Referenzprojektion nur mäßige Rückgang der Flächenneuinanspruchnahme hängt vor allem mit dem höheren Bedarf an urbanen Grün- und Erholungsflächen zusammen sowie mit der gezielten Berücksichtigung von Grünflächen im Neubau und einer geringeren Ausnutzung von Innenentwicklungspotenzialen. Werden innerstädtische Brachflächen nicht für den Neubau, sondern als Grün- und Erholungsflächen genutzt, werden weitere Baugebiete am Siedlungsrand benötigt, was wiederum einen höheren Bedarf an Erschließungsstraßen und Ausgleichsund Ersatzmaßnahmen nach sich zieht. Die Maßnahmen der Klimaanpassungstrategie führen zu einem weniger effizienten Siedlungsmuster als in der Klimaschutzstrategie, da sie den Siedlungsfreiraum höher gewichten.

In Abb. 4.29 wird deutlich, dass die Flächenneuinanspruchnahme in den Regionen hoch bleibt, die auch in der Referenzprojektion die stärksten Wachstumsregionen sind (Umland von München, Berlin und Hamburg, Emsland, nördliches Münsterland, Rheinland, Oberrhein, Großraum Stuttgart). In Regionen mit ohnehin geringen Zuwachsraten kann die Flächenneuinanspruchnahme dagegen weiter gesenkt werden (z. B. in Mecklenburg-Vorpommern, Sachsen-Anhalt, dem südlichen Niedersachsen, sowie in Teilen von Hessen und Rheinland-Pfalz). Dafür sind vor allem die hohen Leerstandsraten verantwortlich, die im Rahmen der Strategie entweder stärker wiedergenutzt oder zurückgebaut werden.

Die Effekte der Klimaanpassungstrategie hinsichtlich der Verbesserung der thermischen Belastung lassen sich mit dem Indikator „Flächeninanspruchnahme in wärmebelasteten Gebieten" messen. Der Indikator setzt sich aus mehreren gleichgewichteten Teilindikatoren 


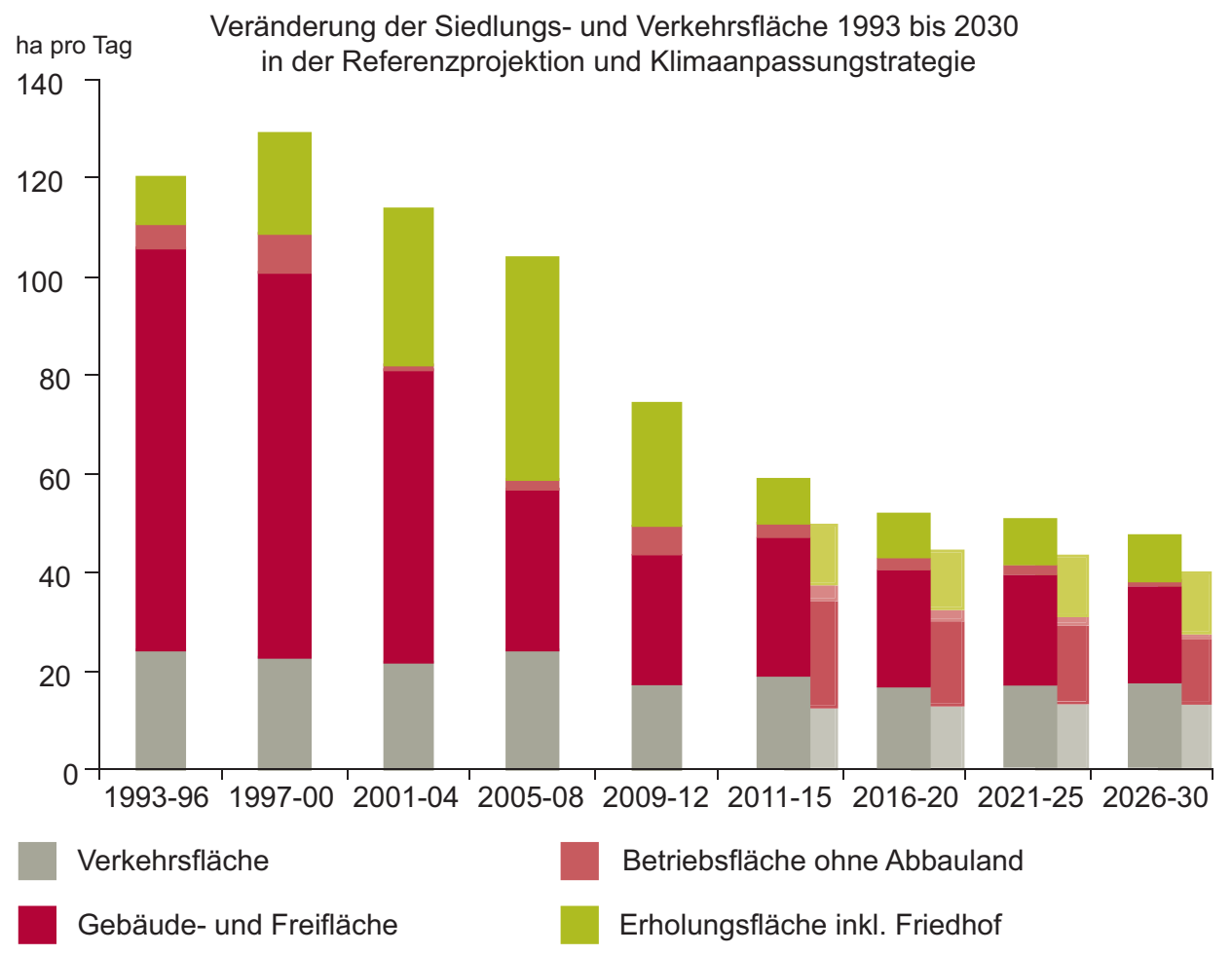

Abb. 4.28 Veränderung der Siedlungs- und Verkehrsflächenentwicklung 1993 bis 2030 für die Referenzprojektion und Klimaanpassungstrategie

zusammen, von denen einer die Versorgung neuer Siedlungsflächen mit erholungsrelevanten Freiflächen beschreibt (Abb. 4.29). Diese verbessert sich durch die eingeleiteten Maßnahmen mit wenigen Ausnahmen in den meisten Kreisen.

Mehrere Maßnahmen der Klimaanpassungstrategie beeinflussen nicht die Höhe der Flächeninanspruchnahme insgesamt, sondern führen $\mathrm{zu}$ einer Verlagerung der Siedlungsentwicklung, v. a. durch die Stärkung des Hochwasserschutzes und weitere Risikovorsorge. Während in der Referenzprojektion das Modell noch 20.000 ha versiegelungsrelevanter Siedlungs- und Verkehrsfläche in Bereichen verortet hat, die von extremen Hochwasserereignissen betroffen sein können, sind es in der Klimaanpassungstrategie 5000 ha weniger bei einer Flächenneuinanspruchnahme, die nur wenig geringer ist als in der Referenzprojektion. In Gebieten mit erhöhter Gefährdung von Hangrutschungen verteilt das Modell in der Referenzprojektion 10.000 ha versiegelungsrelevante Siedlungs- und Verkehrsfläche und in der Klimaanpassungsstrategie 6500 ha. 
a

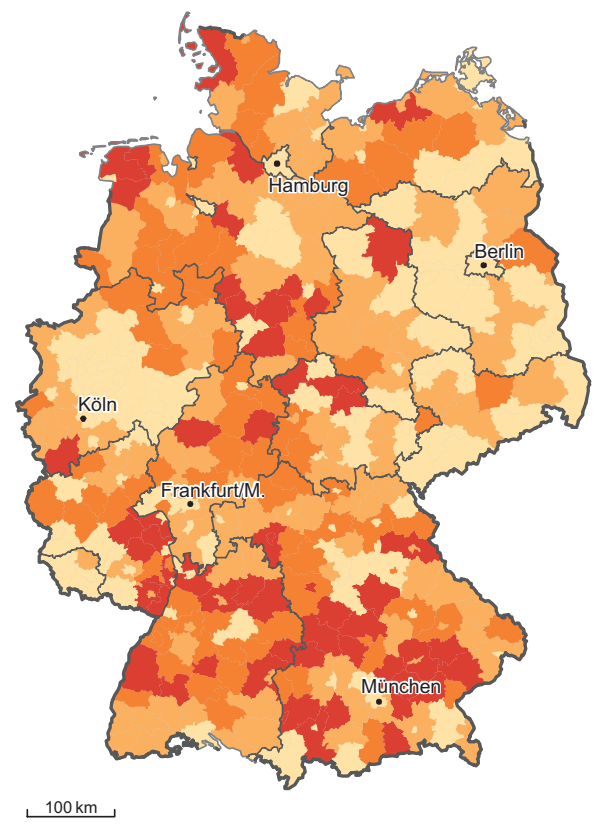

Referenzprojektion

(Abweichung vom Bundesdurchschnitt)

stark unterdurchschnittlich

leicht unterdurchschnittlich

leicht überdurchschnittlich

stark überdurchschnittlch b

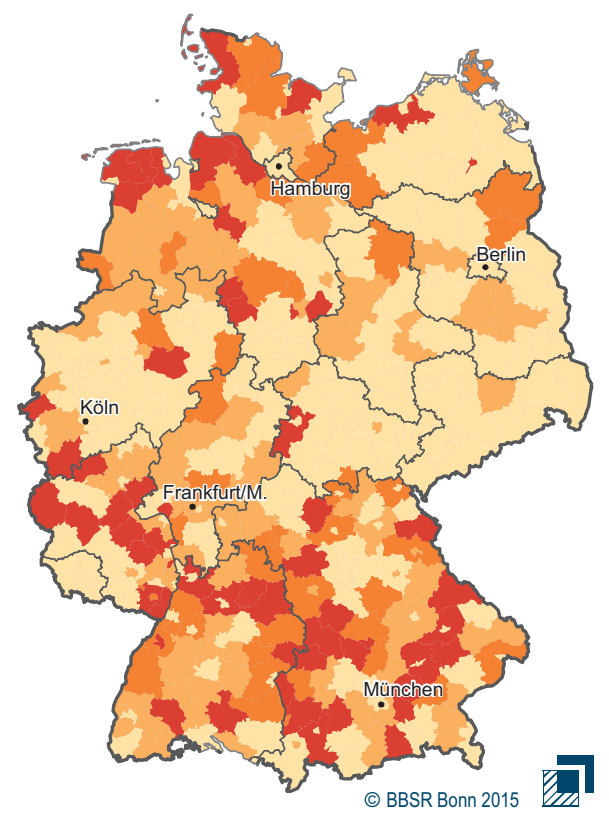

Klimaanpassungstrategie (Abweichung vom Bundesdurchschnitt)

Datenbasis: Laufende Raumbeobachtung des BBSR

Geometrische Datenbasis: BKG/BBSR Kreise, 31.12.2013

Bearbeitung: J. Hoymann, R. Goetzke

Abb. 4.29 Anteil der Siedlungsflächenentwicklung in der Nähe $(500 \mathrm{~m})$ von erholungsrelevanten Freiflächen durch Maßnahmen der Klimaanpassungstrategie im Vergleich zum Bundesdurchschnitt 2009. a Referenzprojektion, b Klimaanpassungstrategie

\subsection{Ergebnisse der landwirtschaftlichen Flächennutzungsmodellierung}

Martin Henseler, Sarah Baum und Peter Kreins

\section{Zusammenfassung}

Die Auswirkungen veränderter agrarpolitischer Rahmenbedingungen und die Umsetzung landwirtschaftlicher Maßnahmen zur THG-Reduzierung wurden mit RAUMIS für das Jahr 2030 simuliert. Hierbei wurden Veränderungen hinsichtlich Einkommen, Produktion, 
THG-Emissionen und andere Umweltfaktoren (Stickstoffdüngung, Pflanzenschutzmitteleinsatz) im Vergleich zur Referenzprojektion analysiert. Die THG-Verringerung ist beim Energiemaisanbau am höchsten, aber durch Intensivierung der Produktion mit negativen Umweltwirkungen verbunden. Aufforstung, Wiedervernässung und Paludianbau haben ebenfalls hohe Einsparpotenziale; flächenbezogen ist dies bei der Aufforstung am höchsten. Die geringsten Vermeidungskosten pro vermiedene Tonne $\mathrm{CO}_{2}$-Äqu. entstehen bei Wiedervernässung, die höchsten bei der verpflichtenden Flächenstilllegung und der Anpassung des Düngemanagements durch eine Stickstoffabgabe. Die Strategien „Klimaschutz“, „Bioenergie“ sowie „Natur- und Umweltschutz“ wurden als Kombination ausgewählter Maßnahmen simuliert. Die Strategie „Bioenergie erzielt deutlich höhere THG-Vermeidungseffekte als die anderen Strategien. Hinsichtlich der durchschnittlichen Vermeidungskosten ist die Strategie „Natur- und Umweltschutz“ am teuersten. Für die Strategie „Klimaschutz“ wurden die geringsten durchschnittlichen Vermeidungskosten pro vermiedener $\mathrm{t} \mathrm{CO}_{2}$ - ̈̈qu. berechnet. Die Strategien wirken sich regional verschieden aus und bedienen die Akteursinteressen unterschiedlich. Die alleinige Betrachtung sektoraler Effekte reicht nicht aus, um die Auswirkungen umfassend abschätzen zu können, was für eine erfolgreiche Umsetzung unumgänglich ist.

\subsubsection{Landwirtschaftliche Referenzprojektion}

Ausgehend vom Basisjahr 2010 wurde bis zum Zieljahr 2030 eine Referenzprojektion simuliert. Sie dient als Vergleichssituation bei der Analyse von Wirkungen einzelner THG-Minderungsmaßnahmen im Bereich der landwirtschaftlichen Landnutzung sowie der oben beschriebenen Landnutzungsstrategien. Die Referenzprojektion basiert auf Annahmen und Ergebnissen der GLUES-Baseline (Delzeit et al. 2016, 2017) sowie der Thünen-Baseline 2013-2023 (Offermann et al. 2014). Die Referenzprojektion stellt wie die Thünen-Baseline keine Prognose der Zukunft dar, sondern beschreibt die erwarteten Entwicklungen unter bestimmten Annahmen zur Entwicklung exogener Faktoren und Politiken. Die Projektion beruht auf den Ende 2013 vorliegenden Daten und Informationen zur weltwirtschaftlichen Entwicklung sowie der Umsetzung der EU-Agrarreform aus dem Jahr 2015.

In der Referenzprojektion wird von einer Beibehaltung der derzeitigen Agrarpolitik bzw. der Umsetzung bereits beschlossener Politikänderungen ausgegangen. Dies beinhaltet im Wesentlichen die folgenden Maßnahmen: die Umschichtung der Mittel der ersten Säule (Direktzahlungen) in die zweite Säule (Förderung der ländlichen Entwicklung), die Entkopplung produktionsbezogener Direktzahlungen als flächenflächenbezogene einheitliche Direktzahlungen, die Verpflichtung der Landwirte zur Einhaltung umweltschonender Flächennutzung („Greening“) sowie das Auslaufen der Milch- und Zuckerquotenregelungen, welche die Produktion vor der Reform beschränkten. Die Förderung der Biogaserzeugung erfolgt durch das EEG, das mehrfach novelliert und 
mit der Novelle im Jahr 2012 deutlich eingeschränkt wurde (vgl. Abschn. 2.1). Für Biogasbestandsanlagen, deren Förderzeitraum vor dem Zieljahr 2030 endet, wird eine Anschlussförderung bis ins Zieljahr 2030 unterstellt, die die Kosten der Biogasherstellung und Stromeinspeisung kompensiert. Die Förderung des EEG-Folgeregimes stellt eine Biogaserzeugung im Umfang der Bestandsanlagen sowie eines moderaten Zubaus bis zum Zieljahr 2030 sicher.

Die Ergebnisse der Thünen-Baseline, die mit Hilfe eines Verbundes unterschiedlicher agrarökonomischer Modelle erstellt werden, wurden mit dem regionalisierten Agrarsektormodell RAUMIS bis zum Jahr 2030 fortgeschrieben. Entwicklungen ausgewählter Betriebsmittelpreise, Produktpreise sowie Flächenerträge sind in Tab. 4.9 zusammengestellt. Die Projektion der Flächenerträge wurde auf der Basis der Ertragszuwächse der letzten 20 Jahre fortgeschrieben. Die Auswirkungen bspw. des Wegfalls von Pflanzenschutzmitteln oder klimatische Änderungen auf die Ertragsrelationen wurden nicht explizit adressiert.

Tab. 4.10 gibt einen Überblick über die projizierten Änderungen in der Pflanzenproduktion und Tierhaltung infolge der agrarpolitischen Rahmenbedingungen sowie Marktentwicklungen. Im Vergleich zum Basisjahr 2010 nimmt die von landwirtschaftlichen Betrieben bewirtschaftete Fläche (LF) aufgrund der anhaltenden Ausdehnung der Siedlungs- und Verkehrsflächen um insgesamt 1,1\% ab (vgl. Abschn. 4.4.1). Innerhalb des Anbaus landwirtschaftlicher Kulturen gewinnen Ölsaaten wegen der vergleichsweise günstigen Marktentwicklungen an Bedeutung. Ihre Anbaufläche wird nach den Modellergebnissen um rund 0,2 Mio. ha ausgedehnt, vor allem zu Lasten des Anbaus von Roggen und Gerste (vgl. Tab. 4.10).

Der Zuckerrübenanbau wird durch den Wegfall der Zuckerquote, kontinuierlich hoher Ertragszuwächse und günstiger Zuckerpreisentwicklungen leicht ausgedehnt

Tab.4.9 Annahmen zur Entwicklung der Produktivität sowie ausgewählter Produktpreise in der Referenzprojektion bis 2030 in Deutschland. (Quelle: Offermann et al. 2014)

\begin{tabular}{l|r|r|r|r}
\hline & \multicolumn{2}{l}{$\begin{array}{l}\text { Basisjahr } \\
\text { 2010 }\end{array}$} & $\begin{array}{r}\text { Referenz 2030 } \\
\text { Betriebsmittelpreise }\end{array}$ & $\begin{array}{l}\text { Änderung 2030 } \\
\text { zu 2010 in \% }\end{array}$ \\
\hline Energie- und Schmierstoffe & $\%$ & 100 & 218 & 218 \\
\hline Düngemittel & $\%$ & 100 & 236 & 236 \\
\hline Maschinen & $\%$ & 100 & 181 & 181 \\
\hline Flächenerträge & $\mathrm{dt} / \mathrm{ha}$ & 73,8 & 91,9 & 24 \\
\hline Weizen & $\mathrm{dt} / \mathrm{ha}$ & 37,1 & 45,3 & 22 \\
\hline Raps & & & & \\
\hline Produktpreise & $€ / t$ & 144 & 209 & 45 \\
\hline Weizen & $€ / t$ & 328 & 419 & 28 \\
\hline Raps & \multicolumn{5}{|l}{}
\end{tabular}


Tab.4.10 Anbauflächen und Produktionsumfänge Tierhaltung im Basisjahr und in der Referenzprojektion 2030. (Quelle: RAUMIS 2017)

\begin{tabular}{|c|c|c|c|c|}
\hline & & $\begin{array}{l}\text { Basisjahr } 2010 \\
\text { (Mio.) }\end{array}$ & $\begin{array}{l}\text { Referenz } 2030 \\
\text { (Mio.) }\end{array}$ & $\begin{array}{l}\text { Änderung } 2030 \mathrm{zu} \\
2010 \text { in \% }\end{array}$ \\
\hline $\begin{array}{l}\text { Landwirtschaftlich } \\
\text { genutzte Fläche }\end{array}$ & ha & 16,9 & 16,7 & $-1,3$ \\
\hline Ackerfläche & ha & 12,0 & 12,2 & 1,9 \\
\hline Weizen & ha & 3,3 & 3,8 & 15,2 \\
\hline Roggen & ha & 0,6 & 0,5 & $-20,6$ \\
\hline Gerste & ha & 1,6 & 1,1 & $-31,1$ \\
\hline Sonst. Getreide & ha & 1,0 & 1,05 & 5,0 \\
\hline Sonst. Marktfrüchte & ha & 0,5 & 0,4 & $-20,8$ \\
\hline Ölsaaten & ha & 1,5 & 1,7 & 14,0 \\
\hline Hackfrüchte & ha & 0,6 & 0,7 & 8,1 \\
\hline Silomais & ha & 1,1 & 1,0 & $-7,6$ \\
\hline Sonst. Ackerfutter & ha & 0,8 & 0,7 & $-2,7$ \\
\hline Energiemais & ha & 0,8 & 1,0 & 23,5 \\
\hline Dauergrünland & ha & 4,9 & 4,5 & $-8,2$ \\
\hline Flächenstilllegung & ha & 0,2 & 0,3 & 31,3 \\
\hline Milchkühe & GVE & 4,2 & 4,1 & $-1,0$ \\
\hline Mastrinder & GVE & 1,8 & 1,7 & $-5,9$ \\
\hline Schweine & GVE & 1,7 & 1,9 & 11,7 \\
\hline Geflügel & GVE & 0,3 & 0,4 & 15,2 \\
\hline
\end{tabular}

(vgl. Tab. 4.10). Das Greening der GAP führt zu einer Ausdehnung der Stilllegungsfläche (+0,08 Mio. ha). Der Wegfall der Milchquote erlaubt bei weiterhin zunehmender Milchleistung je Milchkuh eine Ausdehnung der Milchproduktion bei nahezu konstantem Milchviehbestand. Die stark sinkende Haltung von Mastrindern und sonstigen Rindern (-0,1 Mio. GVE) sowie ein erwarteter Ertragsanstieg bei Silomais bewirken den Rückgang der Silomais- und Grünlandfutterfläche. Eine nachfrageinduzierte Preiserhöhung für Schweinefleisch steigert die Wettbewerbsfähigkeit der Schweinefleischproduktion und führt zu einer Ausdehnung der Schweinehaltung (+0,2 Mio. GVE) bis zum Zieljahr 2030.

Die THG-Emissionen bleiben dabei nahezu konstant $(-0,4 \%)$. Produktivitäts- und Preissteigerung führen zu einem deutlichen Anstieg des landwirtschaftlichen Nominaleinkommens $(+63 \%)$. Unterstellt man die Inflationsraten der Vergangenheit, ergäbe sich ein Anstieg des realen Sektoreinkommens von insgesamt rund $10 \%$ über den gesamten Zeitraum. 


\subsubsection{Analyse ausgewählter landwirtschaftlicher Maßnahmen}

Die Auswirkungen von THG-Minderungsmaßnahmen im Bereich der landwirtschaftlichen Landnutzung wurden mit Hilfe des RAUMIS-Modells (vgl. Abschn. 4.2.2.2) analysiert. Im Folgenden wird die Ausgestaltung der Maßnahmen durch Politikinstrumente sowie die Umsetzung im RAUMIS-Modell erläutert. Das Modell ermittelt eine den veränderten Rahmenbedingungen angepasste optimalere Produktionsstruktur, die mit der Referenzprojektion komparativ statisch verglichen wird.

Die Zahlungen für Ökosystemdienstleistungen (engl. Payments for Ecosystem Services, kurz: PES) sind im RAUMIS-Modell als Anreizinstrument simuliert, um THG-Minderungsmaßnahmen umzusetzen. Dafür wird eine PES in Höhe von $100 € / \mathrm{CO}_{2}$ - ̈̈qu. angenommen. Dieser Wert orientiert sich an den von Schwermer et al. (2012) geschätzten maximalen Schadenskosten, welche bereits in anderen Studien für Mitigationsmaßnahmen in Deutschland als maximale Vermeidungszahlungen angenommen wurden (Röder et al. 2015; Henseler et al. 2015). Diese zum „Europäischen $\mathrm{CO}_{2}$-Handelssystem“ vergleichsweise hohe Mitigationszahlung wird gewählt, um zu analysieren, welche Wirkung des Politikinstruments maximal realisierbar ist. In der Berechnung der Vermeidungskosten werden diese $100 € / t \mathrm{CO}_{2}$ - ̈̈qu. um Mitnahmeeffekte bereinigt. Mitnahmeeffekte entstehen dadurch, dass die PES von $100 € / t \mathrm{CO}_{2}$ Äqu. die tatsächlichen Kosten einiger Maßnahmen deutlich überkompensieren und zu Einkommenssteigerungen im Vergleich zur Referenz führen. Somit werden zur Abschätzung der Vermeidungskosten nur die Kosten betrachtet, die durch die Umsetzung der Maßnahmen entstehen, wie z. B. die Einkommensverluste durch Produktionsverschiebungen und die Kosten der Wiedervernässung.

Die Ergebnisse der Maßnahmewirkungsanalysen sind in einem umfassenden vergleichenden Überblick in Tab. 4.12, 4.13 und 4.14 zusammengestellt.

\subsubsection{Anpassung der Nutzung organischer Böden}

\section{Annahmen und Spezifikation der Maßnahmen}

Die Nutzung organischer Böden wurde in das RAUMIS-Modell als Produktionsverfahren implementiert (vgl. Abschn.4.2.2.2), um die Auswirkungen einer Wiedervernässung auf die landwirtschaftliche Produktion und die THG-Emissionsminderung abzubilden. Zur Berechnung der THG-Emissionen wurden in RAUMIS Emissionsfaktoren aus den Modellen MODE und GAS-EM verwendet (vgl. Abschn. 4.2.2.3).

In Deutschland haben organische Böden einen Anteil von $7 \%$ an der landwirtschaftlichen Nutzfläche (LN) (vgl. Abschn. 2.2.3), was 5,6\% der für RAUMIS relevanten landwirtschaftlich genutzten Fläche (LF) entspricht. Laut Einschätzungen von Experten sind nicht alle entwässerten organischen Böden wiedervernässbar (vgl. Abschn. 3.4.2). Beispielsweise befinden sich zahlreiche organische Böden in einem fortgeschrittenen degradierten Zustand, sodass eine Wiedervernässung nicht mehr möglich ist (Bonn 
et al. 2015). Für die Berechnungen der Vermeidungsleistung wurde unterstellt, dass sich maximal $30 \%$ der organischen Böden wiedervernässen lassen. Ein darüber hinausgehender Wiedervernässungsanteil hielten Experten für unrealistisch.

\section{Sektorale Auswirkungen}

In den Simulationen wird annahmegemäß der maximal mögliche Flächenumfang in Höhe von $30 \%$ der organischen Böden in RAUMIS wiedervernässt. Damit werden insgesamt rund 0,27 Mio. ha (vgl. Tab. 4.12) bzw. rund 1,7 \% der LF aus der Produktion genommen. Die Wiedervernässung der Grünlandfutterfläche reduziert vor allem die darauf basierende Milchkuhhaltung. Neben der THG-Vermeidung führt die Produktionsaufgabe zu einem geringeren Einsatz von Dünge- und Pflanzenschutzmitteln, was weitere positive Umwelteffekte, z. B. hinsichtlich der Biodiversität und der Gewässerqualität, mit sich bringen dürfte.

Das landwirtschaftliche Einkommen geht gemessen an der Nettowertschöpfung zu Faktorpreisen (NWSF) um 0,9\% im Vergleich zur Referenzprojektion zurück. Die Wiedervernässung führt zu einer Reduzierung der deutschlandweiten NettoTHG-Emissionen aus der Landwirtschaft um 7,5\% (vgl. Tab. 4.13). Durch die hohe Vermeidungseffizienz pro Flächeneinheit erzielt die Wiedervernässung eine hohe Vermeidungswirkung von insgesamt 6,0 Mio. $\mathrm{CO}_{2}$ - ̈̈qu. auf lediglich 0,27 Mio. ha wiedervernässter Moorfläche. Die Kosten pro vermiedener Tonne $\mathrm{CO}_{2}$ belaufen sich auf rund $30 €$ (exkl. iLUC-Effekte) und $34 €$ (inkl. iLUC-Effekte; vgl. Tab. 4.14). Die durch iLUC entstehenden THG-Emissionen werden über Schätzwerte nach Osterburg et al. (2013) berüicksichtigt. Die Rechnung basiert auf der Annahme, dass die reduzierte Nahrungsmittelproduktion in Deutschland durch Importe ausgeglichen wird, was zu Intensivierungen und Rodungen in anderen Ländern führen kann.

\section{Regionale Auswirkungen}

Regionale Schwerpunkte des Vorkommens organischer Böden befinden sich in Nordostdeutschland, in Nordwestdeutschland und im zentralen bis südlichen Bayern. Bei der Umsetzung der Maßnahme der Wiedervernässung wird zwischen Acker- bzw. Grünlandnutzung unterschieden. Während organische Ackerflächen vorwiegend im Nordwesten und im Nordosten Deutschlands wiedervernässt werden (vgl. Abb. 4.30), befindet sich wiedervernässtes organisches Grünland in der nordwestdeutschen Küstenregion und in Schleswig-Holstein (vor allem intensives Grünland) sowie, in geringerem Umfang, im südlichen Bayern und Baden-Württemberg (vgl. Abb. 4.30).

Auch wenn die Sektoranalyse die Wiedervernässung als effiziente Maßnahme erscheinen lässt, muss bei der Umsetzung der Förderung dieser Maßnahme die ausgeprägte regionale Heterogenität berücksichtigt werden. Dementsprechend müsste eine Förderung gezielt regional ausgerichtet werden, um Regionen, die die Möglichkeit zur Wiedervernässung haben, einen hinreichend hohen Anreiz zur Wiedervernässung zu bieten. 
a

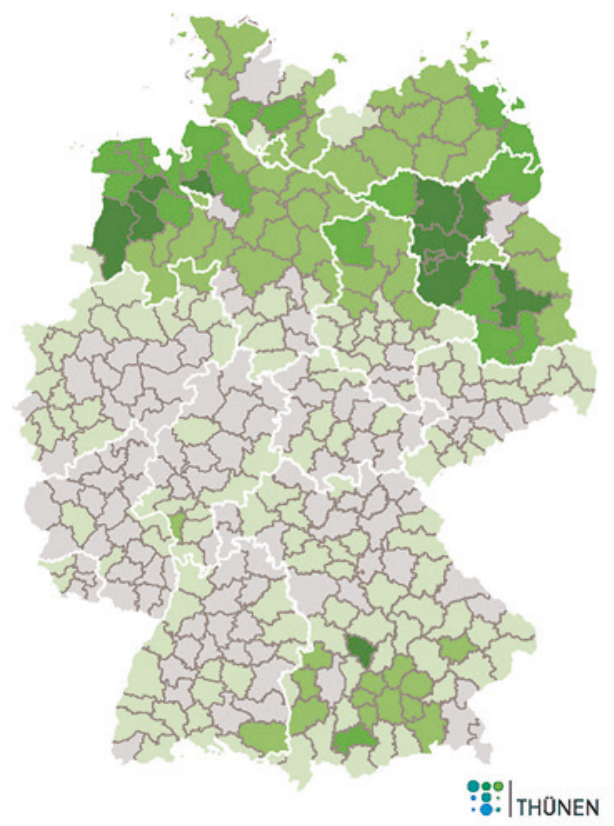

Regionale Umfänge der wiedervernässten Moorackerfläche (\% landwirtschaftliche Fläche)

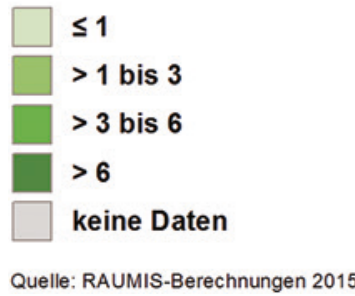

b

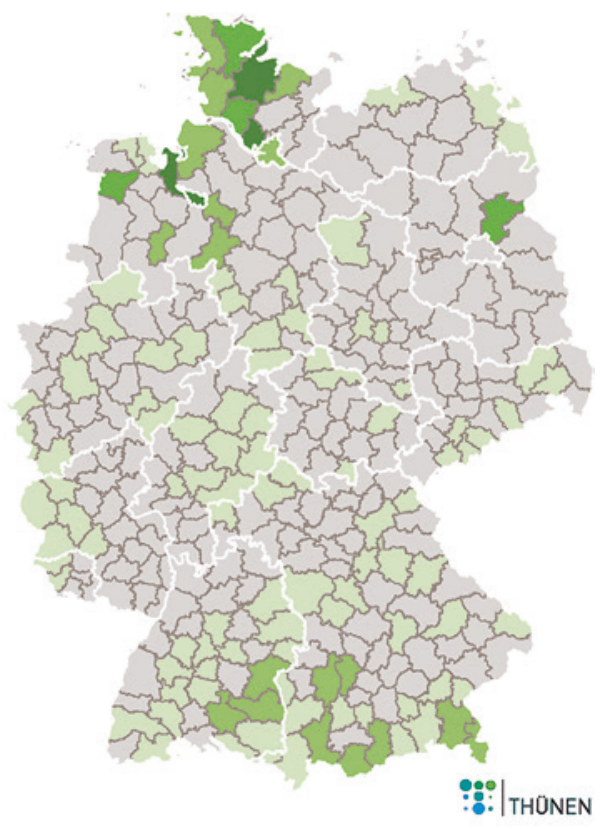

Regionale Umfänge der wiedervernässten Moorgrünlandfläche (\% landwirtschaftliche Fläche)

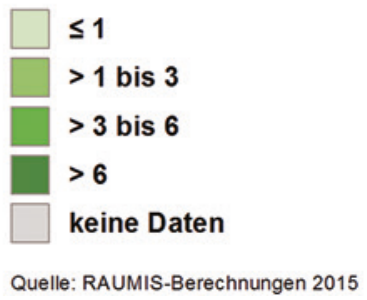

Abb. 4.30 Regionale Umfänge der wiedervernässten Moorackerfläche (\% LF) (a); Regionale Umfänge der wiedervernässten Moorgrünlandfläche (\% LF) (b)

\subsubsection{Anpassung des Düngemanagements}

\section{Annahmen und Spezifikation der Maßnahmen}

Durch Steigerungen der Effizienz des Düngemanagements (vgl. Abschn. 3.4.3) lassen sich THG-Emissionen aus dem Einsatz von Stickstoffdünger vermeiden. RAUMIS bildet die durch Stickstoffdüngung verursachten Lachgas-Emissionen $\left(\mathrm{N}_{2} \mathrm{O}\right)$ aus Mineralböden und organischen Böden ab. Dazu wurden in das Simulationsmodell regional differenzierte Emissionsfaktoren für Ackerlandkulturen und Grünland aus den Modellen 
MODEmin und MODEorg integriert (Abschn. 4.2.2.3). Weitere düngungsabhängige THG-Emissionen werden pauschal bei den indirekten Emissionen berücksichtigt und nicht regional differenziert.

RAUMIS berücksichtigt folgende direkte und indirekte Anpassungen des Düngemanagements:

a) In einem Intensitätsmodul wird die optimale spezielle Bewirtschaftungsintensität, d. h. die optimale Düngermenge in den Anbaukulturen, in Abhängigkeit von Produkt- und Düngemittelpreisen berechnet. Bei einer Veränderung der Relation zwischen Produktund Düngemittelpreisen resultiert eine veränderte optimale Düngeeinsatzmenge.

b) Der gesamtbetriebliche Düngemitteleinsatz wird neben der speziellen Intensität von der Produktionsstruktur beeinflusst. RAUMIS ermittelt bei sich ändernden Rahmenbedingungen eine angepasste optimale Produktionsstruktur. Nimmt beispielsweise der Düngemittelpreis zu, steigt die relative Vorzüglichkeit der düngeextensiveren Ackerkulturen wie Roggen, Sommergerste oder Hülsenfrüchte gegenüber düngeintensiveren Kulturen wie Raps oder Winterweizen.

c) Die Aufgabe der landwirtschaftlichen Produktion umfasst die Flächenstilllegung von Ackerland und Grünland. Auf Stilllegungsflächen finden lediglich Pflegemaßnahmen statt, um die Flächen in einem guten ökologischen und landwirtschaftlichen Zustand zu erhalten.

d) Innerhalb weiter pflanzenbaulicher Grenzen sind in RAUMIS mineralische Dünger und Wirtschaftsdünger substituierbar.

In der Referenzprojektion 2030 beträgt der Anbauumfang von Leguminosen (Hülsenfrüchte, Klee und Luzerne) nur in sehr wenigen Landkreisen mehr als $10 \%$ der LF. Aufgrund der geringen Bedeutung werden Leguminosen nicht explizit als Mitigationsmaßnahme modelliert. Sie sind als Produktionsverfahren in RAUMIS implementiert und können daher grundsätzlich im Zuge von Anpassungen an veränderte Rahmenbedingungen über ihre symbiotische N-Fixierung zur partiellen Substitution von Stickstoffdünger beitragen. In der Grünlandnutzung können intensive Nutzungsformen in extensive Nutzungsformen konvertiert werden, indem die Anzahl der Schnitte oder Weidegänge reduziert wird.

Mit der Reduzierung des Einsatzes von Mineralstickstoffdünger reduzieren sich dessen industrielle Produktion und die damit verbundenen THG-Emissionen. Diese THGVermeidung aus industrieller Produktion wird über einen Emissionsfaktor von 4 t $\mathrm{CO}_{2}$ Äqu./t N (Heinzlmaier 2013) berücksichtigt.

Neben der Einsparung von THG-Emissionen verringert die Reduzierung von Stickstoffdünger das Risiko der Stickstoffeinträge ins Grundwasser sowie in Oberflächengewässer als weitere positive Umweltwirkung.

Eine vielfach diskutierte Stickstoffminderungsmaßnahme ist die Einführung einer Stickstoffabgabe. Hier lassen sich unterschiedliche Ausgestaltungsformen unterscheiden 
(siehe auch Weingarten 1996; Strotmann 1992). So ist beispielsweise eine Abgabe auf mineralische und/oder organische Düngemittel denkbar. Ebenso wird die Einführung einer Abgabe auf den betrieblichen Stickstoffbilanzüberschuss diskutiert. Die Wirkungen der unterschiedlichen Ausgestaltungen unterscheiden sich. In den nachfolgenden Analysen wird eine Abgabe auf mineralischen Stickstoffdünger untersucht, durch die der Preis von Mineralstickstoffdünger um $40 \%$ erhöht wird. Durch die Abgabe wird ein Anreiz gesetzt, den Einsatz von Mineraldünger zu reduzieren.

Die Einführung einer Abgabe auf mineralischen Stickstoff ändert zum einen die Wettbewerbsfähigkeit der Produktionsverfahren in Abhängigkeit von der jeweiligen Einsatzmenge an mineralischem Stickstoff und erhöht zum anderen den Substitutionswert des Stickstoffs in Wirtschaftsdüngern. Infolgedessen wird die Pflanzenproduktion bezüglich der Düngeintensität sowie der Produktionsstruktur angepasst. Die Erhebung einer Mineralstickstoffabgabe von $40 \%$ des Referenzpreises orientiert sich an einer Vorgängerstudie für Deutschland (Henseler und Dechow 2014). Für die Mineralstickstoffabgabe wurde eine produktionsneutrale Rückerstattung angenommen.

\section{Auswirkungen der Maßnahmen}

\section{Sektoranalyse}

Die Einführung einer Stickstoffabgabe führt zu einer Anpassung der Anbaustruktur. Davon betroffen sind hauptsächlich die Weizen- und die Ölsaatenfläche (-7 bzw. $-11 \%)$, während die Silomais- und die Stilllegungsfläche deutlich steigen (+20 bzw. $+160 \%$; vgl. Tab. 4.12) Insgesamt vermindern sich die Netto-THG-Emissionen aus landwirtschaftlichen Böden und Tierhaltung um ca. 4,9 Mio. t $\mathrm{CO}_{2}$ - ̈̈qu. (vgl. Tab. 4.13). Werden die in der industriellen Stickstoffdüngerproduktion eingesparten Emissionen von 1 Mio. t $\mathrm{CO}_{2}$-Äqu. in der Bilanz berücksichtigt, ergibt sich eine Reduzierung der gesamten THG-Emissionen um 5,9 Mio. t $\mathrm{CO}_{2}$ - ̈̈qu.

Die Emissionsminderung resultiert hauptsächlich aus der Pflanzenproduktion, die infolge der maßnahmebedingten Verringerung der Düngeintensität sinkt. Die Erzeugung von Nahrungsmitteln geht um 4,1\% zurück. Die Reduktion des Mineralstickstoffeinsatzes $(-12 \%)$ senkt den Stickstoffbilanzsaldo insgesamt um $5 \%$.

Durch die steigenden Düngekosten und die reduzierte Produktion von Marktfrüchten entstehen hohe Einkommenseinbußen, die sich bei einer Rückführung der erhobenen Stickstoffabgabe als produktionsneutrale Einkommenskompensation auf $5 \%$ reduzieren lassen.

Die Stickstoffabgabe wirkt sich auf die Bewirtschaftungsintensität vermindernd aus und reduziert neben den THG-Emissionen auch den Pflanzenschutzmitteleinsatz. Allerdings bleibt die intensive Tierhaltung erhalten, da keine Besteuerung auf Wirtschaftsdünger vorgenommen worden ist. Die Vermeidungskosten pro vermiedene Tonne $\mathrm{CO}_{2}$ betragen $225 €$ (exkl. iLUC-Effekte) und $381 €$ (inkl. iLUC-Effekte; siehe auch Tab. 4.11). 
Tab. 4.11 Indikatoren zu Einkommen und THG-Emissionen im Basisjahr 2010 und in der Referenzprojektion 2030. (Quelle: RAUMIS 2017)

\begin{tabular}{|c|c|c|c|c|}
\hline & & $\begin{array}{l}\text { Basisjahr } 2010 \\
\text { (Mio.) }\end{array}$ & $\begin{array}{l}\text { Referenz } 2030 \\
\text { (Mio.) }\end{array}$ & $\begin{array}{l}\text { Änderung } 2030 \\
\text { zu } 2010 \text { (in \%) }\end{array}$ \\
\hline $\begin{array}{l}\text { Nettowertschöpfung zu } \\
\text { Faktorkosten }\end{array}$ & EUR & 15.900 & 26.000 & 63 \\
\hline THG-Bilanz gesamt netto ${ }^{a}$ & $\mathrm{t} \mathrm{CO}_{2}$ - ̈̈qu. & 73,0 & 72,6 & $-0,5$ \\
\hline $\begin{array}{l}\text { THG-Emissionen aus } \\
\text { Tierhaltung }{ }^{\mathrm{b}}\end{array}$ & $\mathrm{t} \mathrm{CO}_{2}$ - $\mathrm{A} q u$ & 28,9 & 27,8 & $-3,7$ \\
\hline $\begin{array}{l}\text { THG-Emissionen aus } \\
\text { Mineralböden }^{\mathrm{c}}\end{array}$ & $\mathrm{t} \mathrm{CO}_{2}$ - $\mathrm{Äqu}$ & 19,0 & 19,1 & 0,6 \\
\hline $\begin{array}{l}\text { THG-Emissionen aus } \\
\text { organischen Böden }^{\mathrm{d}}\end{array}$ & t $\mathrm{CO}_{2}$ - $\mathrm{Äqu}$ & 21,7 & 21,7 & 0,00 \\
\hline Indirekte THG-Emissionen ${ }^{\mathrm{e}}$ & $\mathrm{t} \mathrm{CO}_{2}$ - ̈̈qu. & 11,5 & 12,7 & 10,4 \\
\hline
\end{tabular}

aTHG-Bilanz gesamt netto $=$ THG-Emissionen aus Tierhaltung + THG-Emissionen aus Mineralböden+THG-Emissionen aus organischen Böden+Indirekte THG-Emissionen maßnahmebedingter Mitigationseffekt (Wiedervernässung, Substitution)

${ }^{b}$ Emissionen aus Verdauung und Wirtschaftdüngermanagement: $\mathrm{N}_{2} \mathrm{O}$ und $\mathrm{CH}_{4}$

${ }^{\mathrm{c}}$ Emissionen aus der Pflanzenproduktion auf Mineralböden durch mineralisch und organische Düngung: $\mathrm{N}_{2} \mathrm{O}$

${ }^{d}$ Emissionen aus landwirtschaftlich genutzten organischen Böden: $\mathrm{N}_{2} \mathrm{O}, \mathrm{CO}_{2}$ und $\mathrm{CH}_{4}$ e'Lachgasemissionen $\left(\mathrm{N}_{2} \mathrm{O}\right)$ enstammen aus reaktiven Stickstoffverbindungen $\left(\mathrm{NO}_{3}, \mathrm{NH}_{4}\right)$, die aus der landwirtschaftlichen Produktionskette entweichen und in außerlandwirtschaftlichen Systemen in $\mathrm{N}_{2} \mathrm{O}$ umgewandelt werden

\section{Regionalanalyse}

Die regionalen Produktionsintensitäten und -schwerpunkte der Ausgangsituation bestimmen im Wesentlichen das Potenzial der möglichen Minderung des Stickstoffeinsatzes. Die Stickstoffabgabe wirkt in weiten Teilen Deutschlands reduzierend auf den Mineralstickstoffeinsatz (Abb.4.31a) und führt ebenso zu einer großflächigen Reduzierung der Stickstoffflächenbilanz (Abb. 4.31b).

\subsubsection{Substitution fossiler Energie durch Bioenergie}

\section{Annahmen und Spezifikation der Maßnahmen}

Die bei der Verbrennung fossiler Energieträger entstehenden THG-Emissionen lassen sich vermeiden, indem fossile Energie durch Bioenergie substituiert wird. Im RAUMISModell wird die Bioenergieproduktion durch die Verfahren Energiemais für die Biogaserzeugung und Paludikulturen (Abschn. 3.4.2.3) abgebildet. Aus der EEG-Förderung bzw. der Anschlussförderung für Bestandanlagen Biogaserzeugung resultiert das Niveau der Biogaserzeugung und Energiemaisanbau der Referenzprojektion. Um fossile Energie stärker durch Bioenergie zu substituieren, wird als zusätzlicher Anreiz zur Ausdehnung 
a

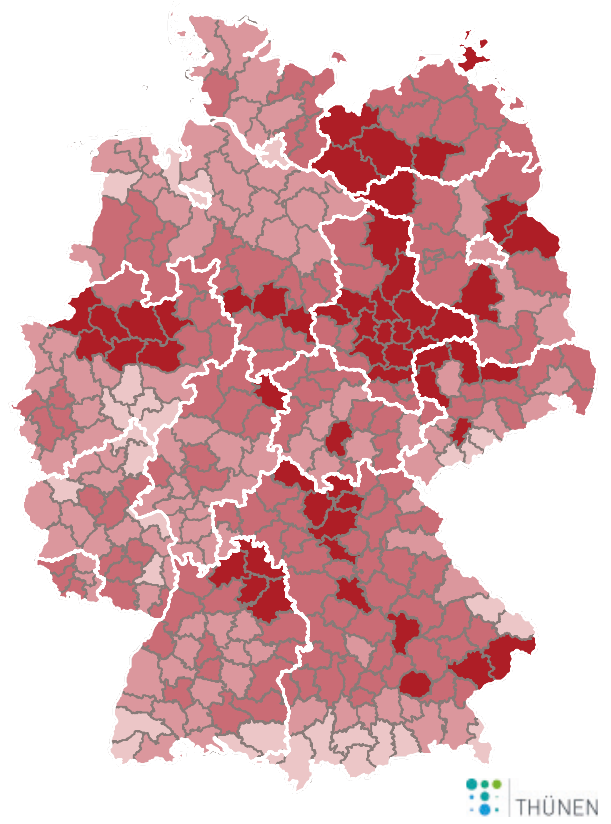

Regionale Änderung des Einsatzes von mineralischem Stickstoffdünger in $\mathrm{kg} \mathrm{N} \mathrm{ha}^{-1}$

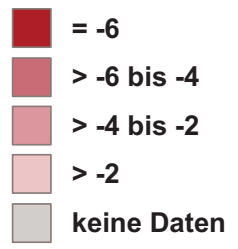

Quelle: RAUMIS-Berechnungen 2015 b

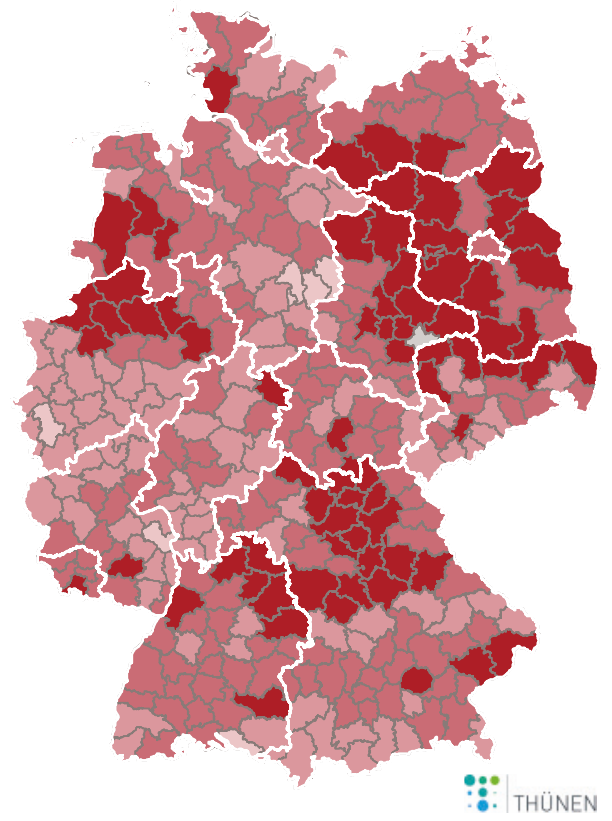

Regionale Änderung der Stickstoffflächenbilanz in $\mathrm{kg} \mathrm{ha}^{-1}$

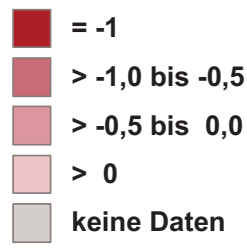

Quelle: RAUMIS-Berechnungen 2015

Abb. 4.31 Regionale Änderung des Einsatzes von mineralischem Stickstoffdünger in $\mathrm{kg} \mathrm{N} \mathrm{ha}^{-1}$ landwirtschaftlich genutzter Fläche (a), Regionale Änderung des Stickstoffflächenbilanzsaldos in $\mathrm{kg} \mathrm{ha}^{-1}$ landwirtschaftlich genutzter Fläche (b)

der THG-mindernden Anbauverfahren eine Direktzahlung in Höhe von $100 € / \mathrm{CO}_{2}$ - $\mathrm{Äqu}$. gezahlt.

Eine zusätzliche Förderung der Produktion von Biokraftstoffen (Bioethanol, Biodiesel) aus Getreide, Zuckerrüben oder Raps wird nicht simuliert. Zum einen sind die Landnutzungseffekte der Biokraftstoffförderung in Deutschland vergleichsweise gering, weil die Produkte der gleichen Anbaukulturen entweder zur Nahrungs- oder zur Biokraftstofferzeugung verwendet werden können. Zum anderen wird sich in der aktuellen politischen 
Debatte weitgehend von der Förderung von Biokraftstoffen abgewandt, weil deren Erzeugung in Deutschland nur eine vergleichsweise geringe THG-Vermeidungsleistung zugeschrieben wird (WBA 2007). Des Weiteren wird in der Projektion von steigender Nahrungsmittelnachfrage und somit steigenden Agrarpreisen ausgegangen (vgl. Abschn. 3.4.4). Daher wird auf die Simulation einer Förderung von Biokraftstoffen, welche den Preisdruck auf Agrarprodukte deutlich erhöhen würde, verzichtet. Die Entwicklung der Biokraftstoffnachfrage ist indirekt über die Preisentwicklung für Ölsaaten in der Referenzprojektion berücksichtigt.

Der einjährige Energiemais steht in Konkurrenz zu anderen Ackerkulturen (z. B. Getreide, Ölsaaten und Ackerfutter). Die maximale Ausdehnung der mehrjährigen Paludikultur wird auf landwirtschaftlich genutzte An- und Niedermoorstandorte außerhalb der Schutzgebietskulisse (kein Nationalpark, Naturschutzgebiet, FFH-, SPAoder Ramsar-Gebiet) beschränkt. Ferner wird der Wiedervernässungsanteil auf maximal $30 \%$ der organischen Böden begrenzt. Es wird angenommen, dass die Paludiproduktion gleichermaßen auf wiedervernässtem Acker- und Grünland umgesetzt wird.

Die Wiedervernässung organischer Böden ist die Voraussetzung für den Anbau von Paludikulturen. Daher wird bei der Modellierung der Maßnahmen die Vermeidungsleistung aus der Wiedervernässung als indirekte Vermeidungsleistung in den Gesamteffekt einbezogen. Die Wettbewerbsfähigkeit der Paludiproduktion auf wiedervernässten organischen Böden wird ebenfalls durch Mitigationszahlungen zur Substitution fossiler Energieträger sowie für die Vermeidung von THG-Emissionen aus organischen Böden erhöht.

Die folgenden Berechnungen sind getrennt für eine Förderung des Energiemaisanbaus und von Paludikulturen durchgeführt worden.

\section{Auswirkungen der Maßnahmen}

\section{Sektoranalyse}

Energiemaisanbau: Durch die zusätzliche Mitigationszahlung wird die Energiemaisfläche gegenüber der Referenzprojektion um rund 1 Mio. ha ausgedehnt, zu Lasten des Anbaus pflanzenschutzintensiverer Kulturen (Weizen $-6 \%$ und Ölsaaten $-11 \%$, vgl. Tab. 4.12), wodurch der Pflanzenschutzmitteleinsatz sinkt ( $-3 \%$, vgl. Tab. 4.13). Die verringerte Futtermittelproduktion (Silomais $-4 \%$ ) führt $\mathrm{zu}$ einer geringfügigen Intensivierung der Grünlandnutzung sowie einer reduzierten Tierhaltung (z. B. der Rinderhaltung, vgl. Tab. 4.12), wodurch die Emissionen aus der Tierhaltung zurückgehen (-0,3 Mio. $\mathrm{C} \mathrm{CO}_{2}-$ Äqu.; vgl. Tab. 4.13). Die THG-Gesamtnettovermeidung bezogen auf die Anbaufläche von Energiemais (1 Mio. ha) führt zu einer Flächeneffizienz von 10,9 t CO $\mathrm{CO}_{2}$ - Äqu./ha (exkl. iLUC-Effekte) bzw. 8,5 t $\mathrm{CO}_{2}$-Äqu./ha (inkl. iLUC-Effekte). Die Vermeidungskosten je vermiedener Tonne $\mathrm{CO}_{2}$ - ̈̈qu. belaufen sich auf $183 €$ (exkl. iLUC-Effekte) und auf $234 €$ (inkl. iLUC-Effekte; vgl. Tab. 4.14). Durch die hohe Profitabilität des Energiemaises und dessen Ausdehnung entstehen in der Landwirtschaft keine Einkommensverluste. 
矛

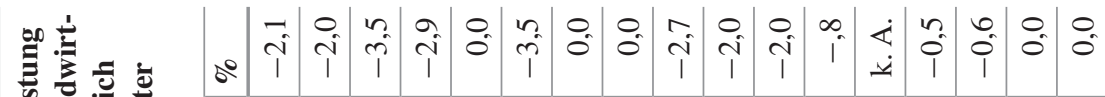

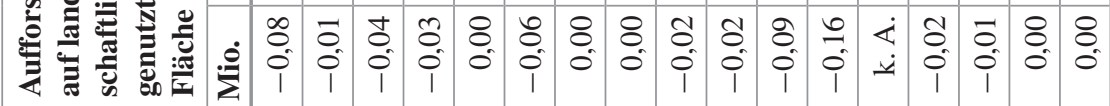
菑

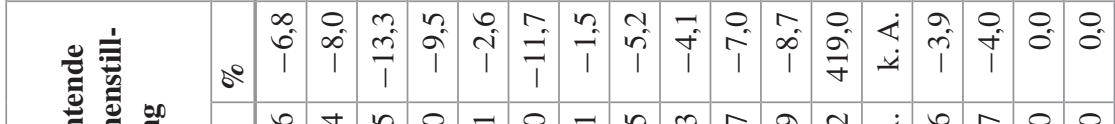

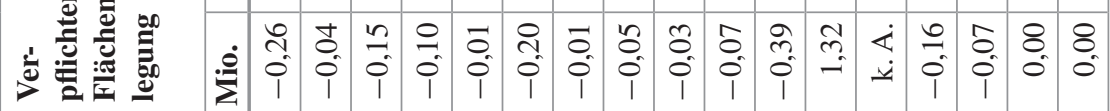
כ

更

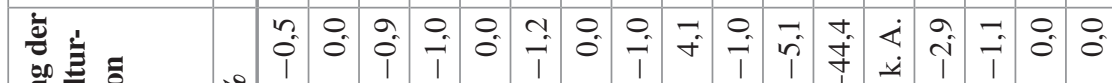

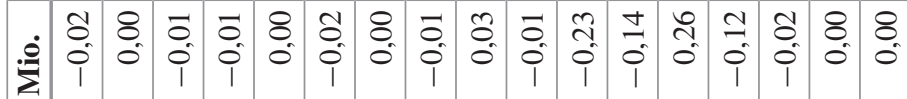

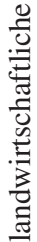

$\because$

光

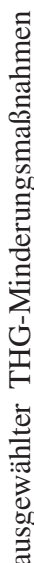

施

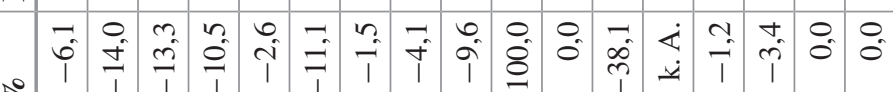

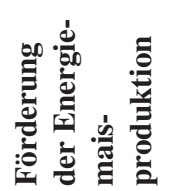

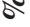

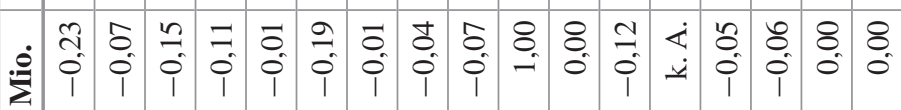

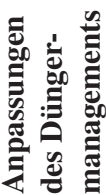
so

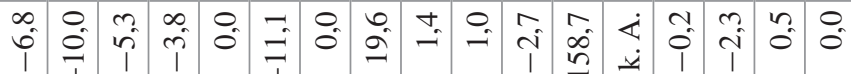

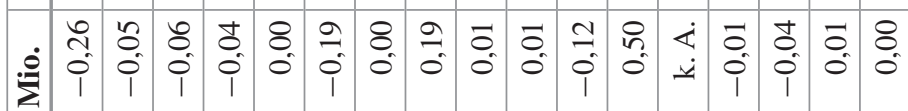

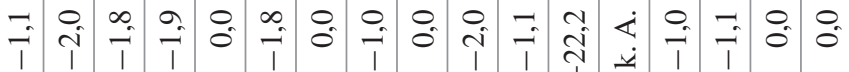
:

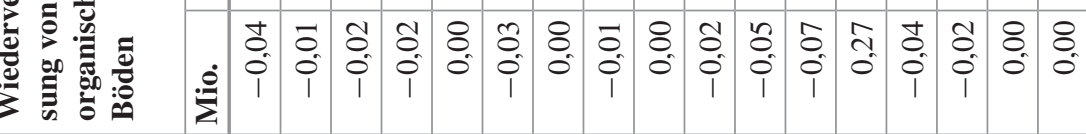

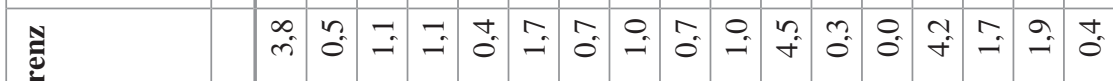

$\dot{\ominus}$

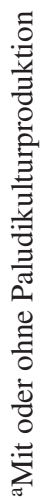




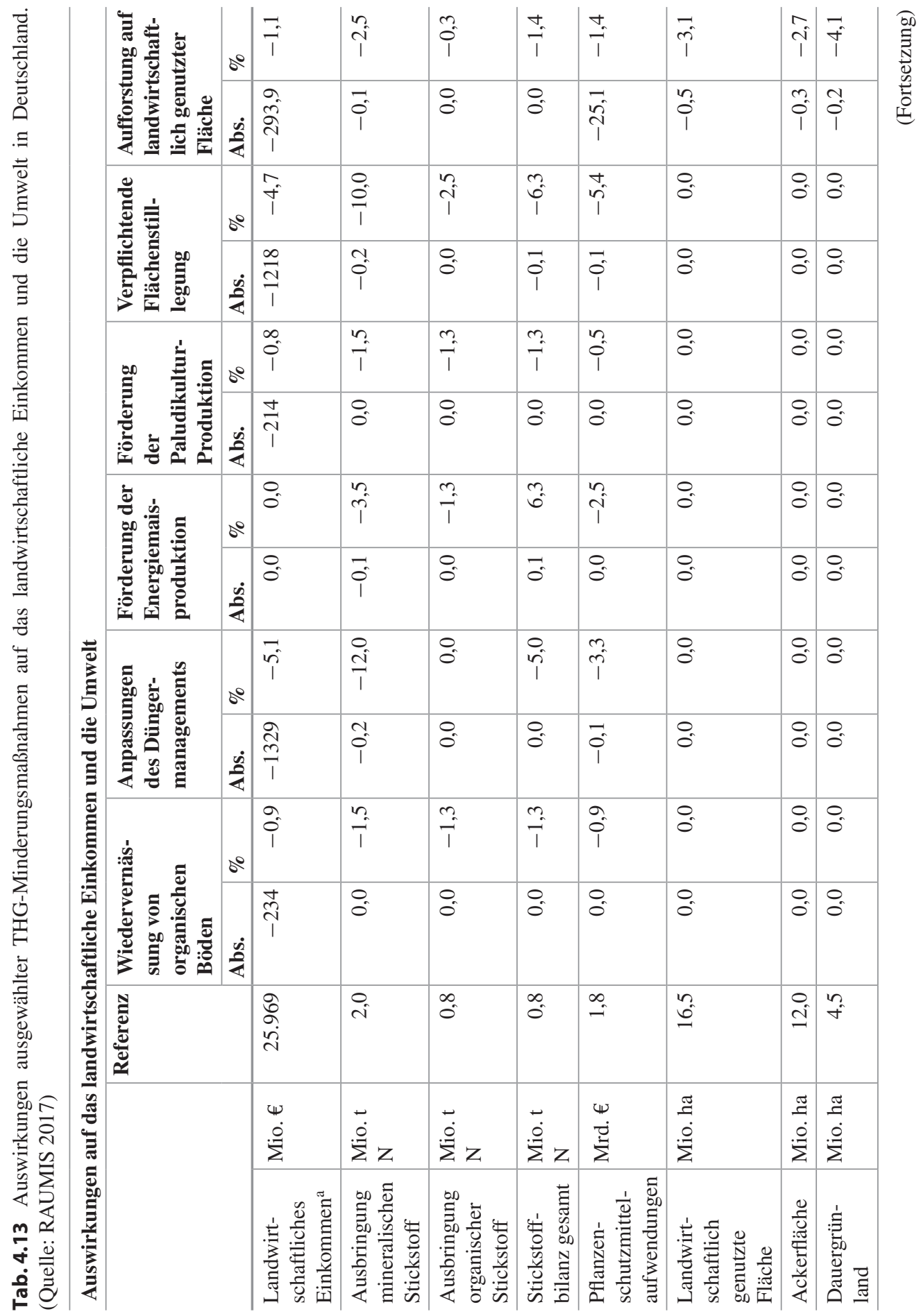




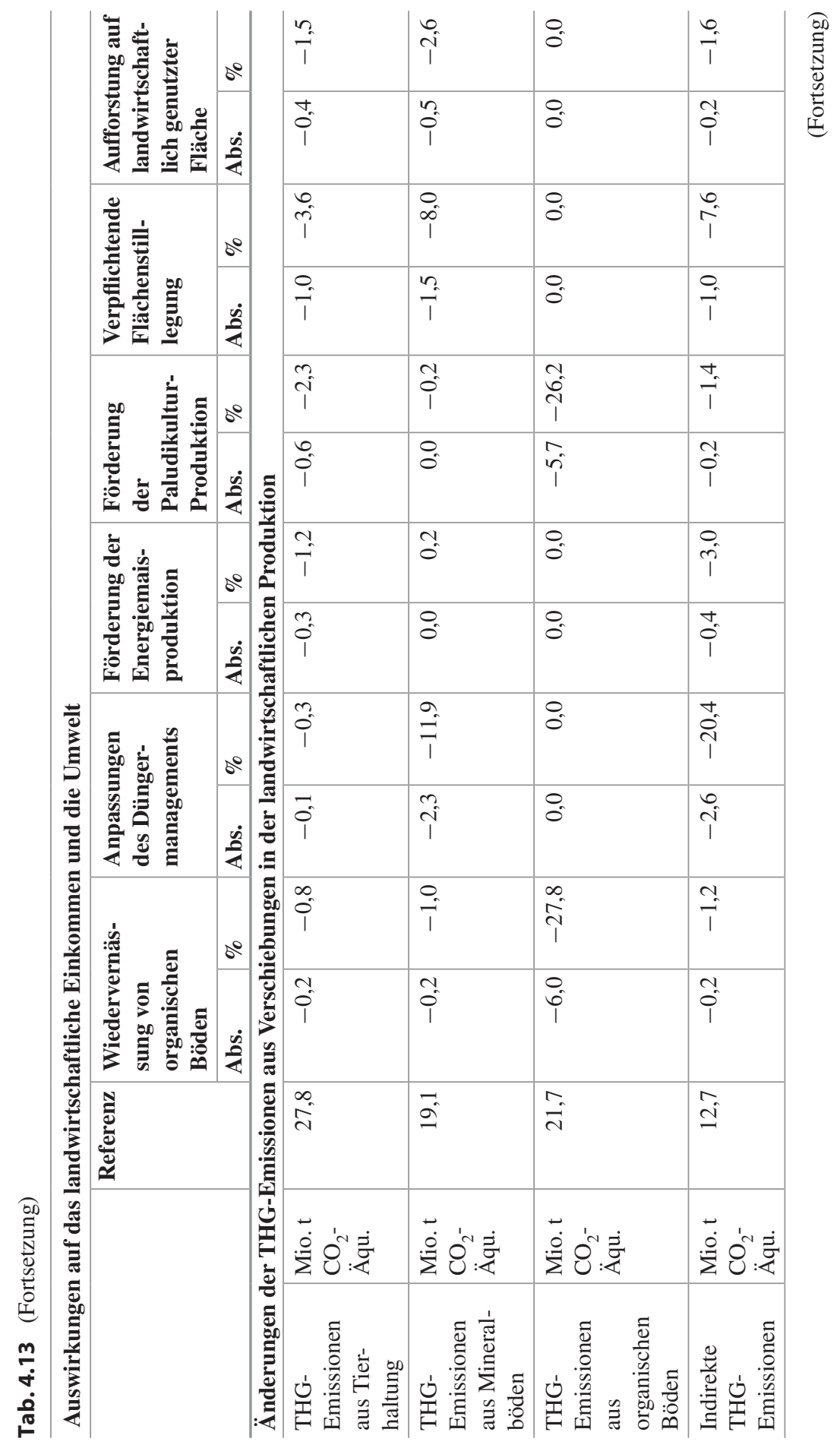




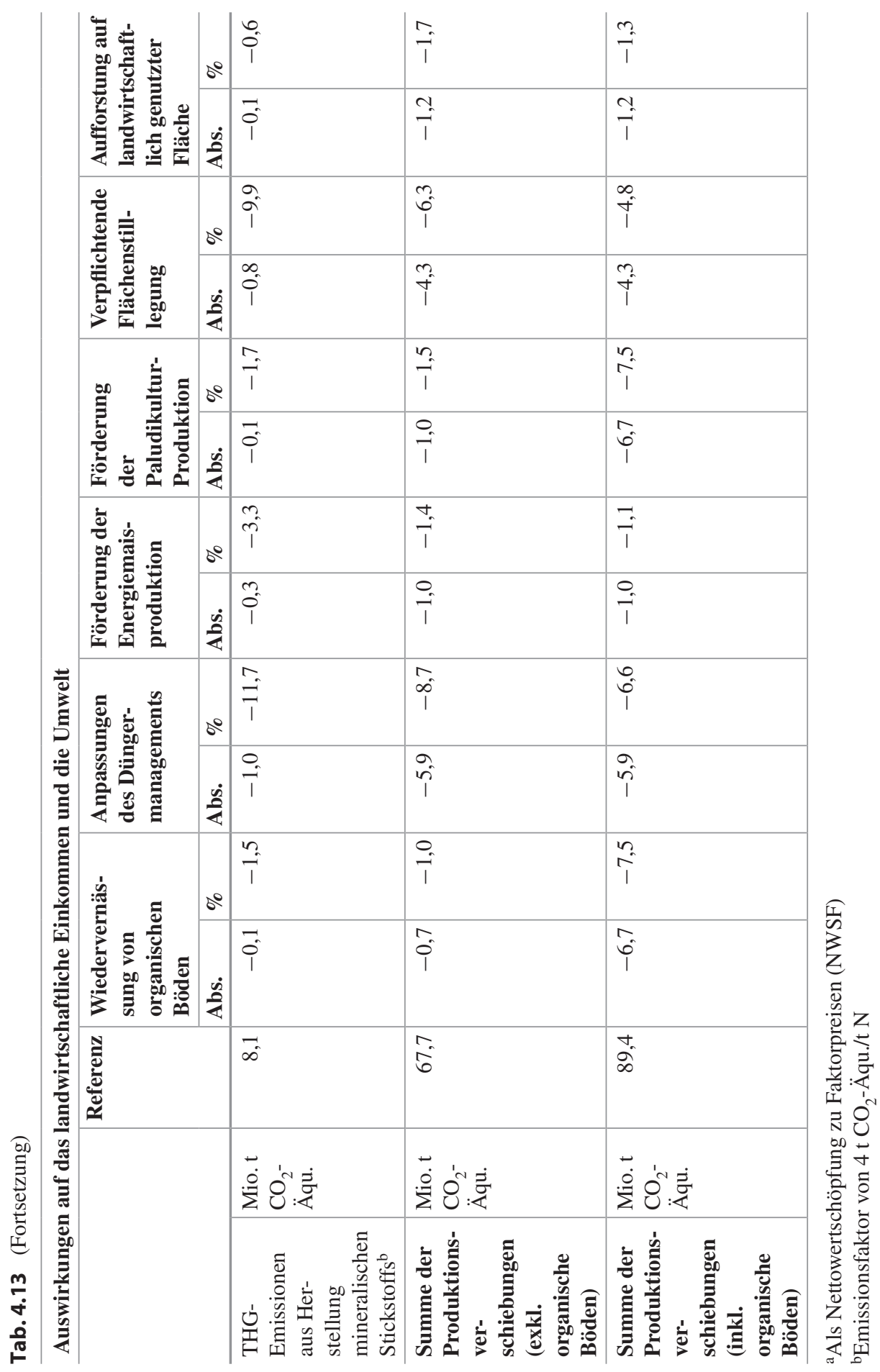




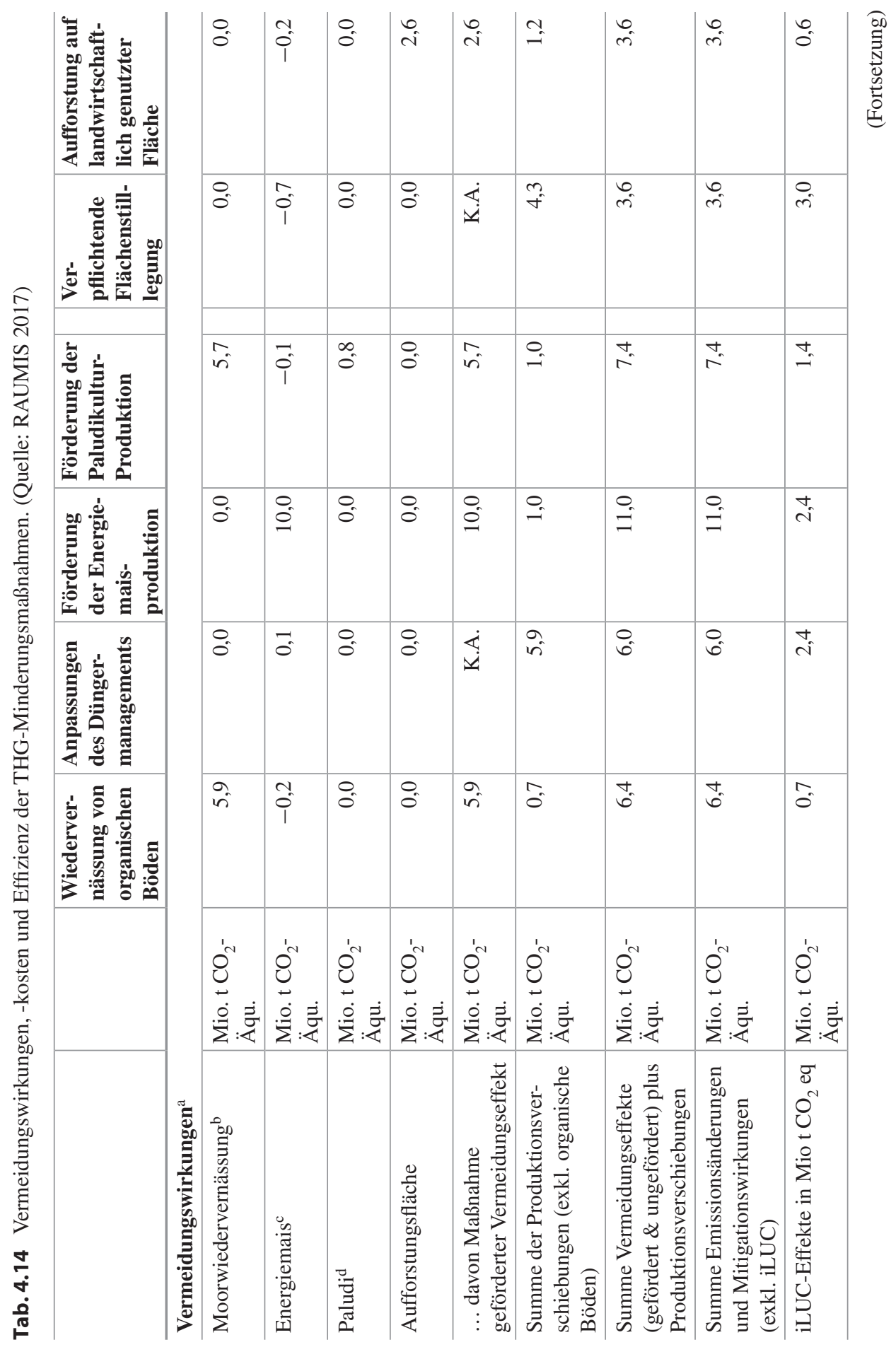




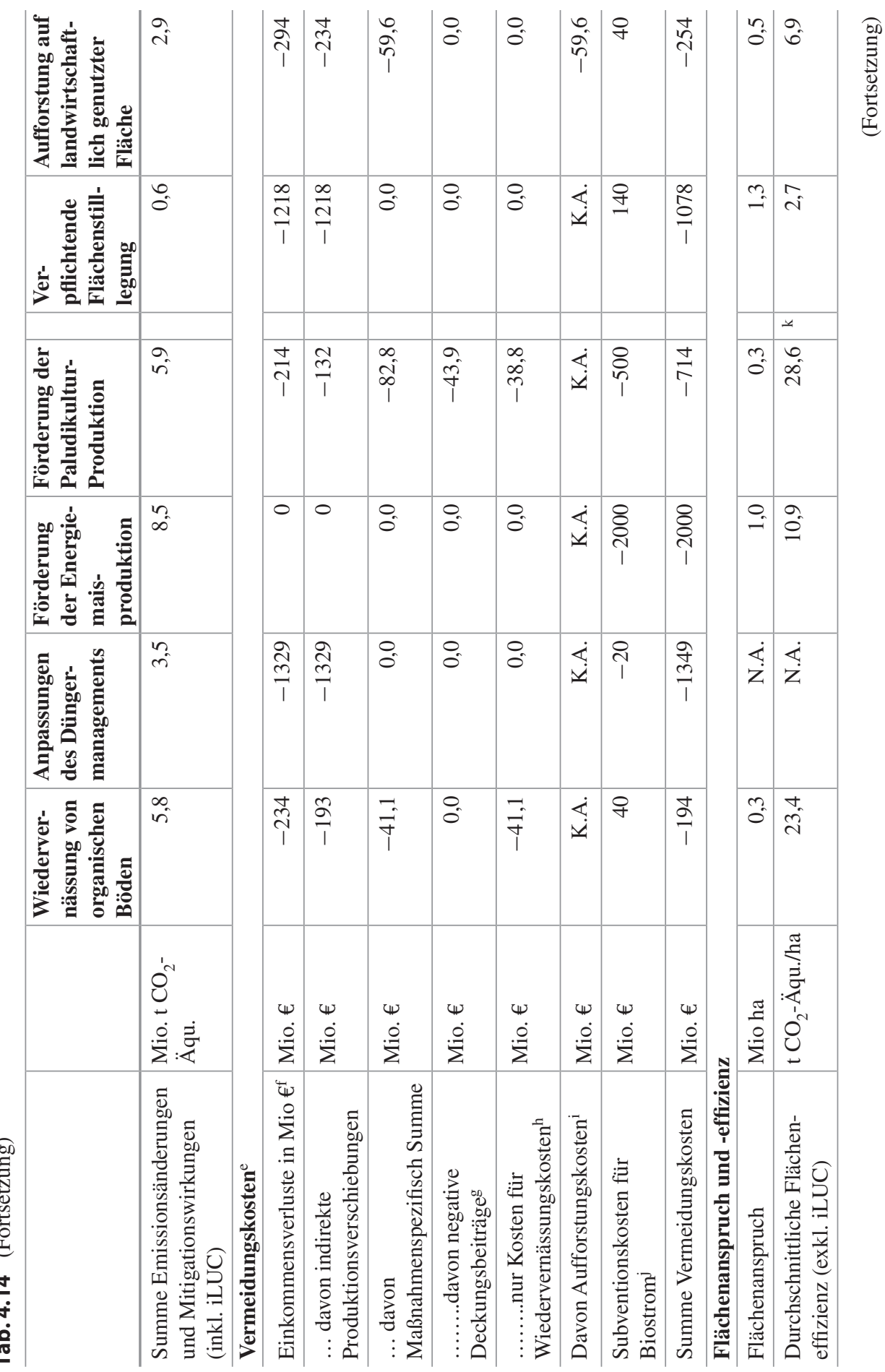


S. Baum et al.

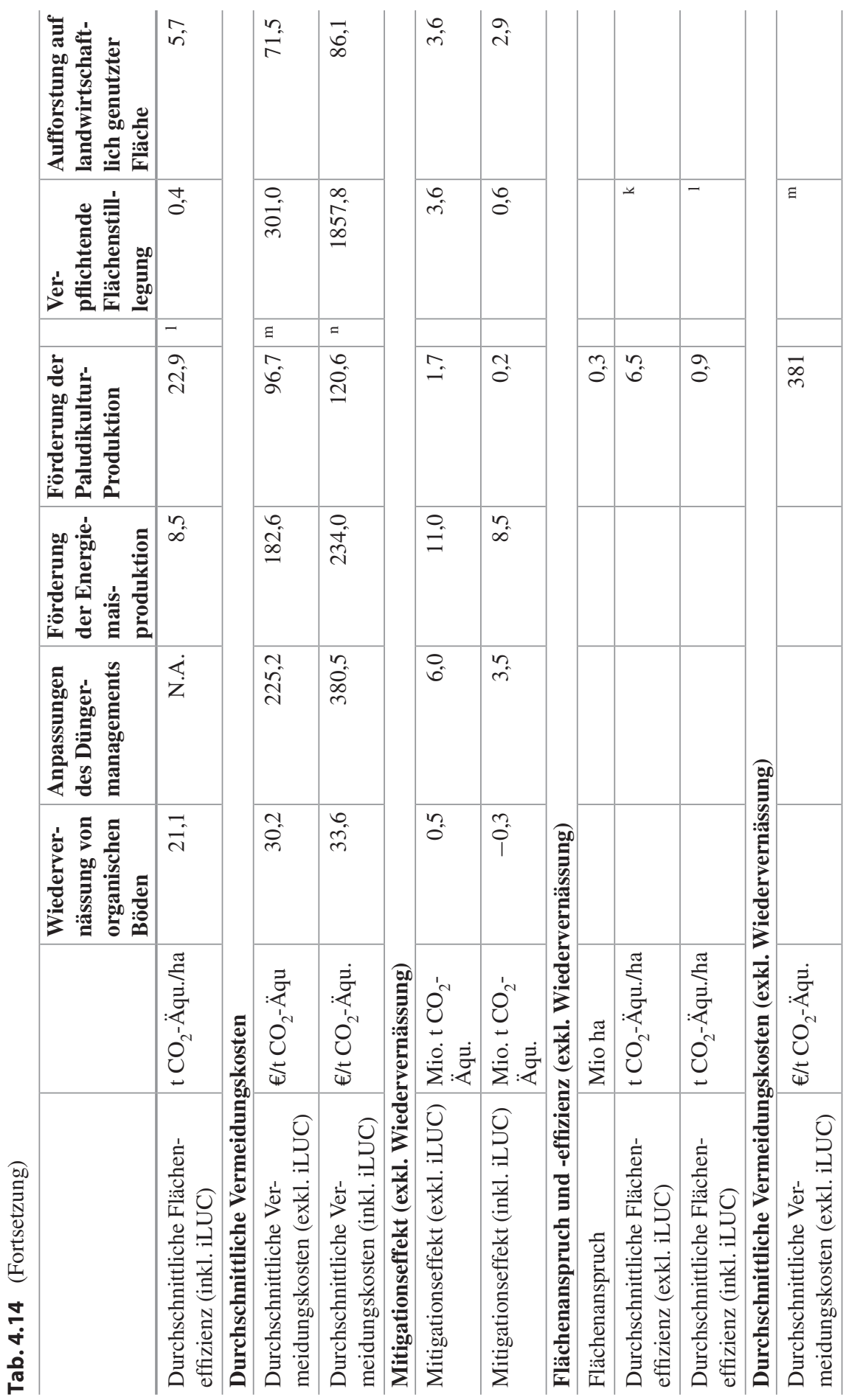




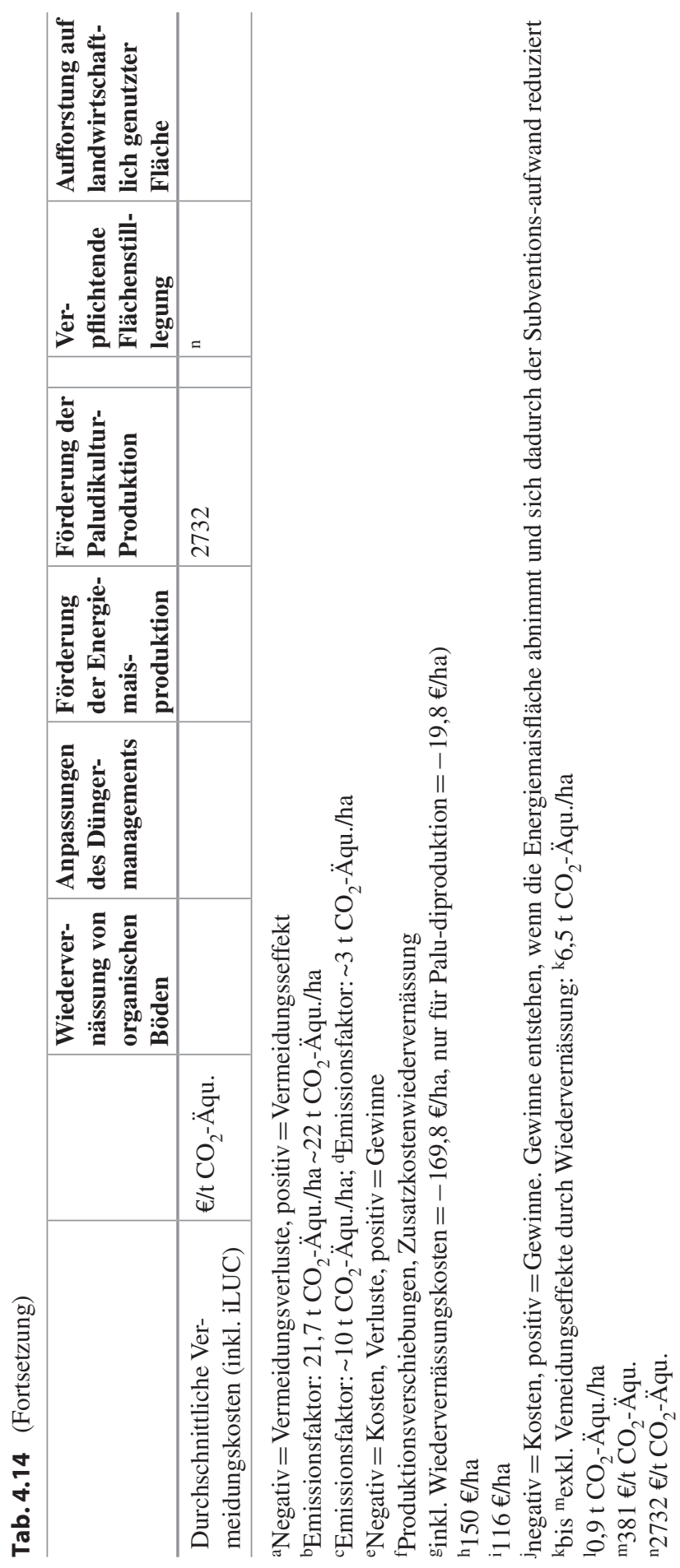


Die Förderung der Substitution fossiler Energien durch Biogas aus Energiemais verringert die Netto-THG-Emissionen um 11 Mio. $\mathrm{CO}_{2}$ - ̈̈qu. (vgl. Tab. 4.14). Da der Energiemais in der Regel mineraldüngerintensivere Kulturen (z. B. Getreide) verdrängt und seinen Düngebedarf überwiegend durch die Gärsubstratrücklieferung deckt, verringert sich der Einsatz von mineralischem Stickstoff (-4\%, vgl. Tab. 4.13). Allerdings weist das Gärsubstrat des Energiemaises, das als organischer Dünger in der Pflanzenproduktion eingesetzt wird, eine geringere N-Effizienz gegen über mineralischem Stickstoffdünger auf, wodurch sich der Gesamtstickstoffbilanzsaldo erhöht (+6\%, vgl. Tab. 4.13) und somit das Risiko zur Nitratauswaschung ins Grundwasser.

Paludianbau: Der Anbau von Paludikulturen ist annahmegemäß auf die wiedervernässten organischen Böden in Höhe von 0,26 Mio. ha (vgl. Tab. 4.12) begrenzt. Der Umfang von Dauergrünland nimmt ab (-5\%), während der Flächenumfang für sonstiges Ackerfutter ansteigt (+4\%). Ein starker Rückgang ist bei der Flächenstilllegung $(-44 \%) \mathrm{zu}$ beobachten, da diese Flächen genutzt werden zur Produktion von Paludikulturen und zur Produktion von Nahrungs- und Futtermitteln, um die Produktionsverluste durch wiedervernässte Standorte abzufangen (vgl. Tab. 4.12).

Die regionale Verdrängung der intensiven Verfahren durch die extensiven Paludikulturen bewirkt eine Reduzierung der Düngeintensitäten und eine sinkende N-Flächenbilanz $(-1 \%)$ sowie einen zurückgehenden monetären PSM-Einsatz $(-1 \%)$. Aufgrund verlorener Futterflächen wird die Tierhaltung reduziert, was einen Rückgang der THG-Emissionen aus der Tierhaltung um 0,6 Mio. t $\mathrm{CO}_{2}$-Äqu. bewirkt (vgl. Tab. 4.14).

Die energetische Nutzung der Paludibiomasse zur Substitution fossiler Brennstoffe trägt zum Vermeidungseffekt nur 0,8 Mio. t $\mathrm{CO}_{2}$ - ̈̈qu. bei, da die Emissionsvermeidungsleistung pro Flächeneinheit mit rund $3 \mathrm{t} \mathrm{CO}_{2}$-Äqu./ha relativ gering ist (vgl. Tab. 4.14). Die aggregierten Vermeidungseffekte, ohne die Vermeidungsleistung der Wiedervernässung organischer Böden, betragen daher nur 1,5 Mio. t $\mathrm{CO}_{2}$-Äqu. (vgl. Tab. 4.10). Werden nur die der Paludiproduktion zugeordneten Kosten (aus Produktionsverschiebungen und Biostromsubvention) betrachtet, ergeben sich vergleichsweise hohe Vermeidungskosten von $381 € / t \mathrm{CO}_{2}$-Äqu. (exkl. iLUC-Effekte). Berücksichtigt man die iLUC-Effekte von 1,4 Mio. t $\mathrm{CO}_{2}$-Äqu., übersteigen die Kosten sogar $2000 € / t \mathrm{CO}_{2}$-Äqu.

Die Wiedervernässung organischer Böden ist die Voraussetzung zur Paludiproduktion und trägt zur Reduzierung der Emissionen mit 5,7 Mio t $\mathrm{CO}_{2}$ - ̈̈qu. bei. Werden zur Bewertung der Maßnahme die Vermeidungseffekte der energetischen Nutzung und die der Wiedervernässung organischer Böden betrachtet, betragen die Vermeidungskosten $97 \mathrm{EUR} / \mathrm{t} \mathrm{CO}$ - ̈̈qu. (exkl. iLUC-Effekte) und $121 \mathrm{EUR} / \mathrm{CO}_{2}$-Äqu. (inkl. iLUCEffekte).

\section{Regionalanalyse}

Energiemaisanbau: Die Umsetzung der Maßnahme der Produktion von Energiemais zur Bioenergiegewinnung ist deutschlandweit verteilt. Dabei wird der Energiemaisanbau in allen Regionen zumindest leicht ausgedehnt, jedoch schwerpunktmäßig in 
Regionen, in denen die Energiemaisproduktion in der Referenzprojektion bereits eine hohe Wettbewerbsfähigkeit aufweist (im Nordwesten, Nordosten, Südwesten, Bayern, vgl. Abb. 4.32a). In diesen Regionen kann der erhöhte Energiemaisanbau zu negativen Umweltwirkungen führen (erhöhter Stickstoffaustrag, erhöhtes Erosionspotenzial). Des Weiteren wird die Nahrungsmittelproduktion in diesen Regionen durch die Bioenergieproduktion verdrängt.

Paludianbau: Die Umsetzung der Maßnahme „Bioenergiegewinnung aus Paludikulturen" wird durch das regionale Vorkommen der An- und Niedermoore bestimmt. Produktionsschwerpunkte ergeben sich im Nordwesten, Nordosten sowie in Südbayern (vgl. Abb. 4.32b). Daher sind die Auswirkungen durch eine verstärkte Energieproduktion aus Paludikulturen regional begrenzt.

\subsubsection{Erhalt und Schaffung von Treibhausgassenken}

\section{Annahmen und Spezifikation der Maßnahmen}

RAUMIS berücksichtigt drei flächenbasierte Maßnahmen zur Erhalt und Schaffung von THG-Senken (Kohlenstoff-Sequestrierung): a) Obligatorische Flächenstilllegung, b) Aufforstung auf landwirtschaftlich genutzten Flächen und c) Erhalt des Grünlands.

a) Die obligatorische Flächenstilllegung ist zum einen eine Maßnahme, um die durchschnittliche Düngeintensität bzw. die durch die Düngung bedingten LachgasEmissionen zu reduzieren (vgl. Abschn. 2.3). Zum anderen erhält bzw. schafft eine begrünte Stilllegungsfläche eine THG-Senke. RAUMIS differenziert Flächenstilllegung in zwei Verfahren: Stilllegungsfläche auf Ackerfläche und auf Dauergrünland, wobei jeweils die Erhaltung der Fläche in gutem landwirtschaftlichen und ökologischen Zustand unterstellt wird. Durch die Flächenstilllegung von Ackerund Grünlandfläche könnte als indirekte Auswirkung eine Steigerung der Bewirtschaftungsintensität in anderen Regionen Deutschlands (oder auch weltweit) zur Kompensation der Produktionsverluste erfolgen (iLUC-Effekt).

Die Maßnahme der obligatorischen Flächenstilllegung wird in der Simulation auf $10 \%$ der landwirtschaftlich genutzten Fläche umgesetzt. Dabei kann die Flächenstilllegung wahlweise auf Acker- und/oder Grünlandfläche erfolgen. Die Gemeinsame Agrarpolitik (GAP) der EU schreibt im Rahmen des Greening seit dem 01.01.2015 für Betriebe mit über 15 ha Ackerfläche vor, dass $5 \%$ ihrer Ackerfläche als ökologische Vorrangflächen ausgewiesen werden müssen. Die Landwirte können zwischen verschiedenen Flächentypen mit Nutzung (z. B. Kurzumtriebsplantagen, Leguminosen, Zwischenfruchtanbau) oder ohne Nutzung (z. B. Brachflächen, Landschaftselemente) wählen, wobei die Flächentypen unterschiedlich angerechnet werden. RAUMIS setzt das Greening als Flächenstilllegung um. Die Reduktion der landwirtschaftlichen Produktion auf den stillgelegten Flächen führt zur Minderung der THG-Emissionen sowie des Einsatzes von Stickstoffdüngern und Pflanzenschutzmitteln. Dadurch wird die Belastung für Flora und Fauna sowie Gewässer und Böden reduziert. Den Stilllegungsflächen wird eine Funktion als Habitat zur Förderung der Biodiversität zugeschrieben. 
a

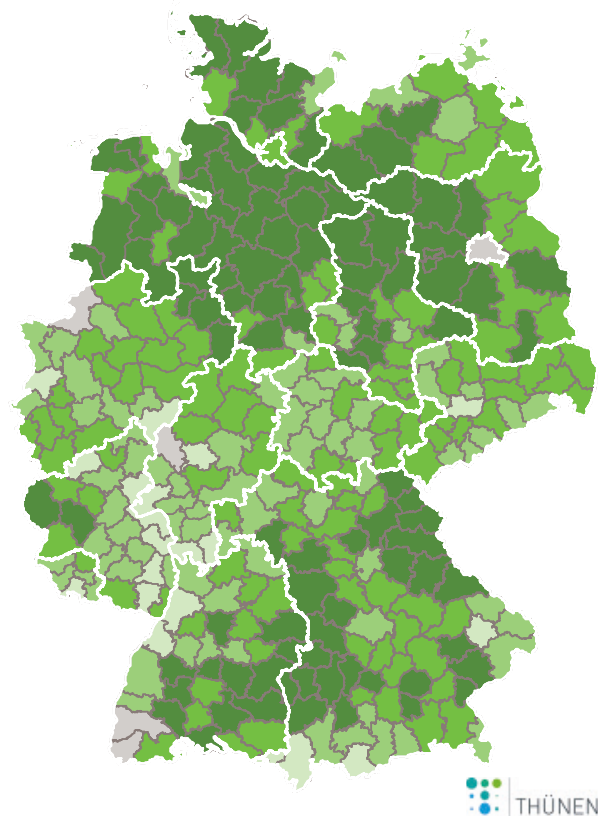

Regionale Änderung der Energiemaisproduktion in \%-Punkten der landwirtschaftlichen Fläche (LF)

$\begin{aligned} & \square<=1 \\ &>1 \text { bis } 3 \\ &>3 \text { bis } 6 \\ &>6 \\ & \text { keine Daten } \\ & \text { Quelle: RAUMIS-Berechnungen } 2015\end{aligned}$ b

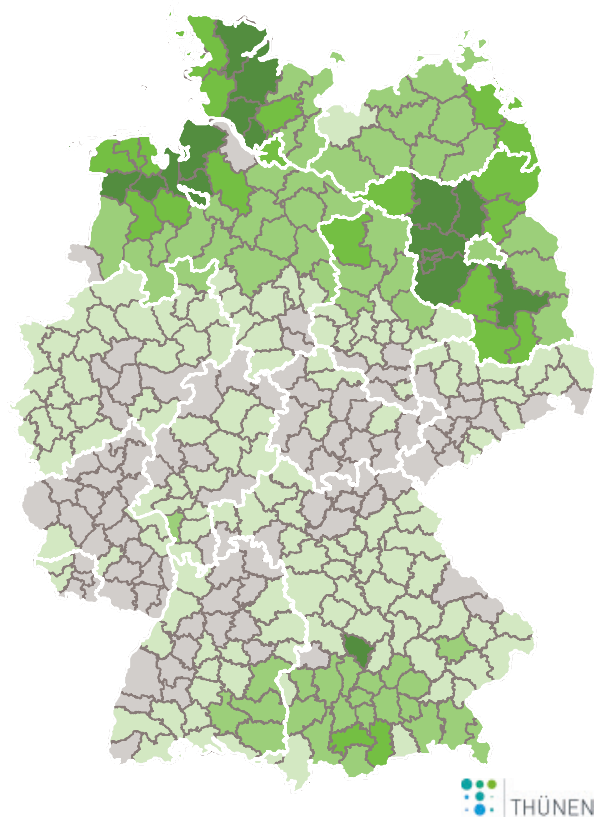

Regionale Änderung der Paludikulturproduktion in \%-Punkten der landwirtschaftlichen Fläche (LF)

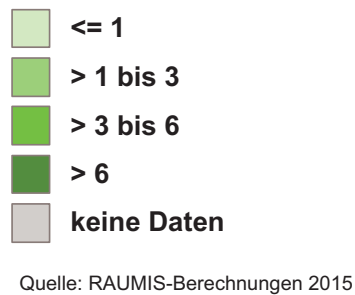

Abb. 4.32 Regionale Änderung der Energiemaisproduktion in Prozentpunkten der landwirtschaftlich genutzten Fläche (a), Regionale Änderung der Paludikulturproduktion in Prozentpunkten der landwirtschaftlich genutzten Fläche (b)

b) Die Aufforstung auf landwirtschaftlich genutzten Flächen stellt eine Schaffung von THG-Senken dar, da der Atmosphäre durch das Holzbiomassewachstum auf den neuen Forstflächen $\mathrm{CO}_{2}$ entzogen wird. Die katastermäßig erfasste Landwirtschaftsfläche (LN) ist um ca. 2 Mio. ha größer als die durch die Agrarstatistik erfasste landwirtschaftlich genutzte Fläche (LF), die als Datenbasis für RAUMIS dient. Über die Nutzung dieser Flächen liegen keine belastbaren Informationen vor, da sie überwiegend durch statistisch nicht erfasste Betriebe (z. B. kleine Betriebe unterhalb der Erfassungsgrenze, Pferde- 
haltung) und private landwirtschaftliche Nutzung (z. B. private Pferdehaltung, Streuobstwiesen) bewirtschaftet werden. In der Vergangenheit fand ein Teil der Neuaufforstung auch auf diesen Landwirtschaftsflächen statt, die nicht durch die Agrarstatistik erfasst werden.

Durch die Aufforstung soll die Waldfläche um $10 \%$ bzw. 1,08 Mio. ha ausgedehnt werden (vgl. Abschn. 4.4.3). Diese Flächenvorgabe resultiert aus der modellexogenen Flächenkulisse für 2030 des Modells Land Use Scanner (vgl. Abschn. 4.2.2.1). Es wird angenommen, dass rund die Hälfte (0,51 Mio. ha) der Aufforstung auf der in RAUMIS berücksichtigten LF auf Grenzertragsstandorten umgesetzt wird. Für die andere Hälfte wird unterstellt, dass die Aufforstung auf nicht von der Agrarstatistik erfassten Landwirtschaftsflächen erfolgt.

Durch die Konvertierung von LF zu Forstfläche verzeichnen die Produzenten Flächenund Einkommensverluste auf den ehemaligen Agrarflächen. Da RAUMIS keine forstwirtschaftlichen Verfahren abbildet, werden die dortigen Einkommen und Kosten sowie die Mitigationswirkungen durch C-Sequestrierung modellexogen berücksichtigt und in den Analysen für den Forstsektor untersucht (Abschn. 4.6).

c) Der Erhalt von Grünland ist in RAUMIS durch das Verbot von Grünlandumbruch explizit berücksichtigt. Die regionalen Umfänge von Acker- und Grünlandfläche sind als modellexogene Flächenkulissen in RAUMIS abgebildet, die RAUMIS über das Modell Land Use Scanner für alle Strategien bezieht (vgl. Abschn. 4.2.2.1).

\section{Sektorale Auswirkungen der Maßnahme}

Obligatorische Flächenstilllegung: Wird der Anteil der obligatorischen Flächenstilllegung auf $10 \%$ der LF festgelegt, sind gegenüber der Referenzprojektion zusätzlich rund 1,3 Mio. ha der Anbauflächen stillzulegen. (Dies entspricht $8 \%$ der LF). Die Anbauumfänge nehmen vor allem bei Dauergrünland (-9\%), Weizen $(-7 \%)$, Energiemais $(-7 \%)$ und Ölsaaten $(-12 \%)$ ab (vgl. Tab. 4.12). Da vorrangig ertragsschwächere Standorte stillgelegt werden, nimmt die landwirtschaftliche Produktion unterproportional ab. Zwar werden die Stickstoffdüngung und der PSM-Einsatz absolut reduziert, die verbleibenden Produktionsflächen weisen jedoch eine durchschnittlich höhere Produktionsintensität auf.

Der maßnahmebedingte Rückgang der Pflanzen- und Tierproduktion führt zu einer vergleichsweise geringen Reduzierung der THG-Emissionen um 3,6 Mio. t $\mathrm{CO}_{2}$ - ̈̈qu. (exkl. iLUC-Effekte) und um 0,6 Mio. t $\mathrm{CO}_{2}$-Äqu. (inkl. iLUC-Effekte; (vgl. Tab. 4.13). Den stillgelegten Flächen wird eine Funktion als $\mathrm{CO}_{2}$-Speicher zugeschrieben, da kein Umbruch erfolgt. Diese THG-Emissionsminderung wird vorrangig den Mineralböden zugeschrieben, da der absolute Umfang der Flächenstilllegung auf organischen Böden gering ist.

Angesichts der zusätzlichen Stilllegung von $8 \%$ der LF, sinkt das landwirtschaftliche Einkommen nur um $5 \%$, da vor allem ertragsschwächere Standorte aus der Produktion genommen werden. Bezogen auf die maßnahmebedingt stillgelegten Flächen von 1,3 Mio. ha beträgt die Flächeneffizienz 2,7 $\mathrm{CO}_{2}$-Äqu./ha (exkl. iLUC-Effekte) und 
0,4 t $\mathrm{CO}_{2}$ - ̈̈qu./ha (inkl. iLUC-Effekte). Für die obligatorische Flächenstilllegung fallen Vermeidungskosten von $301 € / \mathrm{CO}_{2}$ - $\mathrm{Äqu}$. an. Werden die iLUC-Effekte berücksichtigt, belaufen sich die Vermeidungskosten auf mehr als $1000 € / \mathrm{t} \mathrm{CO}_{2}$ - ̈̈qu.

Aufforstung in Höhe von $10 \%$ der Waldfläche auf landwirtschaftlicher Nutzfläche: Die landwirtschaftlich genutzte Fläche (LF) wird annahmegemäß um 3,1 \% (0,51 Mio. ha) verringert (vgl. Tab. 4.13). Die Hälfte der Fläche entfällt vor allem auf freiwillig stillgelegte Flächen und Grünland. Durch die mit der Flächenumwidmung einhergehenden Verringerungen der landwirtschaftlichen Produktion nehmen die THG-Netto-Emissionen um 3,6 Mio. t $\mathrm{CO}_{2}$ - ̈̈qu. ab (vgl. Tab. 4.13).

Die Senkenfunktion der aufgeforsteten Fläche wird der landwirtschaftlichen Fläche zugeordnet. Die kumulierte Flächeneffizienz der im Jahr 2015 aufgeforsteten Fläche beträgt im Jahr 2030 und 80,5 t CO${ }_{2}$ - ̈̈qu./ha; im Jahr 2055 wäre es mit 398,3 t CO $2^{-}$ Äqu./ha bereits fünfmal so viel. Die mittlere annuelle Senkenfunktion der Aufforstungsfläche auf der LF beträgt für den Betrachtungszeitraum von 2015 bis 2030 rund $5 \mathrm{t}$ $\mathrm{CO}_{2}$ - ̈̈̈qu./ha und entspricht somit einer jährlichen Vermeidungsleistung von 2,6 Mio.t $\mathrm{CO}_{2}$ - Äqu. Betrachtet man die aufgeforstete LF für den gesamten forstwirtschaftlichen Simulationszeitraum (2015 bis 2055), ist die annuelle Vermeidungsleistung deutlich größer und beläuft sich auf 7,1 t CO $2-$ Äqu./ha bzw. insg. 3,7 Mio. t $\mathrm{CO}_{2}$ - ̈̈qu.

Entsprechend der Vermeidungseffekte werden auch die Einkommensverluste der landwirtschaftlichen Aktivitäten $(-1 \%)$ mit Einnahmen und Ausgaben aus der Forstwirtschaft auf den ehemaligen Landwirtschaftsflächen verrechnet. Da es sich um eine Douglasien-Neuanpflanzung handelt (vgl. Abschn. 4.6), entstehen zunächst hohe Kosten, die die Erlöse übersteigen. Im betrachteten Zeitraum von 2015 bis 2030 entstehen auf der aufgeforsteten LF Kosten in Höhe von 59,6 Mio. € (entsprechend durchschnittlich annuell $116 € /$ ha). Bei der Berechnung der Aufforstungskosten wurden die Investitionskosten der Anpflanzung zu gleichen Teilen auf 80 Jahre verteilt. Ein Betrachtungszeitraum von 16 Jahren ist für einen Wald ein kurzer Zeitraum, vor allem, wenn es sich wie hier um eine Neuanpflanzung handelt. Die Umtriebszeit (Uz) von Douglasien beträgt ca. 80 Jahre. Neuanpflanzungen erfolgen mit hoher Pflanzdichte, sodass im Laufe der Jahre vereinzelt junge Bäume entnommen werden müssen, um den verbleibenden Bäumen gute Wuchsbedingungen zu ermöglichen. Durch diese Entnahmen entsteht zwar ein Gewinn; dieser kann die hohen Anpflanzungskosten jedoch zunächst nicht decken. Für das Simulationsjahr 2030 betragen die durchschnittlichen Vermeidungskosten je Tonne $\mathrm{CO}_{2}{ }^{-}$ Äqu. $72 €$ (exkl. iLUC-Effekte) und um $87 €$ (inkl. iLUC-Effekte).

\section{Regionalanalyse}

Die obligatorische Flächenstilllegung als Maßnahme zur Emissionsreduzierung wird deutschlandweit umgesetzt, hat aber regional unterschiedliche Auswirkungen auf den Umfang und die Art der Flächenstilllegung. Je nach regionaler Vorzüglichkeit und bereits vorhandener Flächenstilllegung wird entweder Ackerland oder Grünland aus der landwirtschaftlichen Produktion genommen. Im Nordosten sind bereits in der Referenzprojektion hohe Flächenanteile stillgelegt, sodass nur noch wenig Fläche zusätzlich aus 
der Nutzung genommen werden muss, um die vorgeschriebenen $10 \%$ der LF zu erfüllen (vgl. Abb. 4.33a). In Nordrhein-Westfalen, Rheinlandpfalz und im Südwesten werden bedeutende Teile des Grünlands stillgelegt. Im Nordwesten und Bayern hat das Grünland eine wichtige Bedeutung als Futterbasis für die Rinderhaltung, weshalb hier vorwiegend extensives Ackerland stillgelegt wird (vgl. Abb. 4.33b), und Grünland als Futterbasis erhalten bleibt.

a

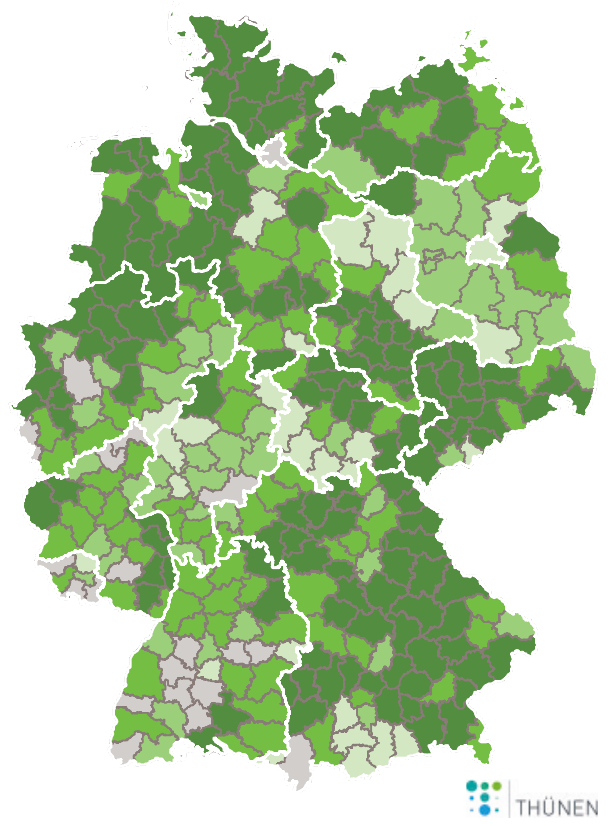

Regionale Änderung der Flächenstilllegung auf Ackerland in \%-Punkten der LF unter der Maßnahme „Verpflichtende Flächenstillegung“

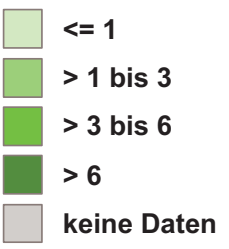

Quelle: RAUMIS-Berechnungen 2015 b

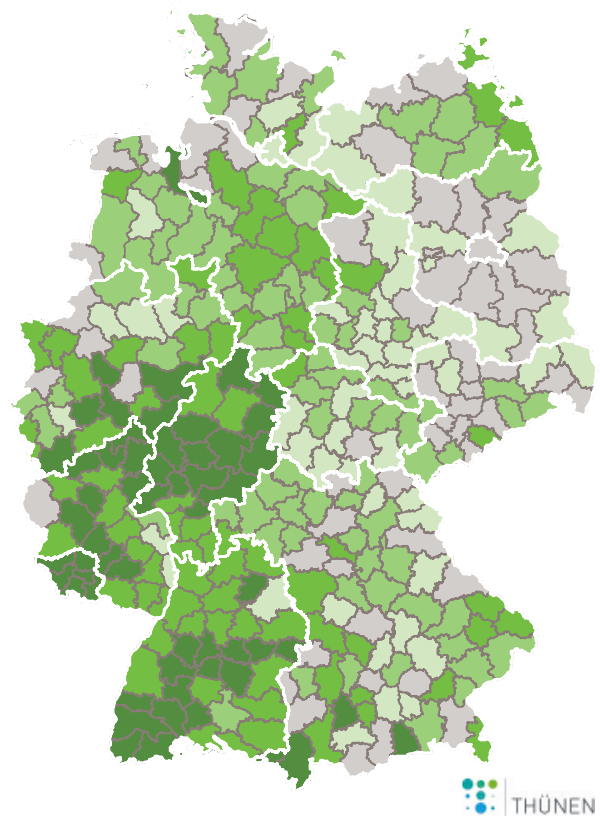

Regionale Änderung der Flächenstilllegung auf Grünland in \%-Punkten der LF unter der Maßnahme „Verpflichtende Flächenstilllegung“

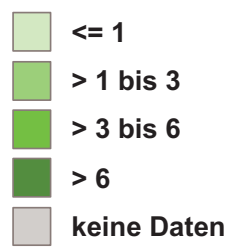

Quelle: RAUMIS-Berechnungen 2015

Abb. 4.33 a Regionale Änderung der Flächenstilllegung auf Ackerland in \% der landwirtschaftlich genutzten Fläche unter der Maßnahme „Verpflichtende Flächenstilllegung“, b Regionale Änderung der Flächenstilllegung auf Grünland in \% der landwirtschaftlich genutzten Fläche unter der Maßnahme „Verpflichtende Flächenstilllegung“ 
Die Umsetzungsschwerpunkte der Aufforstung auf landwirtschaftlicher Nutzfläche liegen vor allem in Südwest-, Mittel- und Teilen Nordostdeutschlands (vgl. Abb. 4.34). In Südwestthüringen werden große Flächenanteile aufgeforstet. Der geringe Anteil der zusätzlichen obligatorischen Flächenstilllegung deutet darauf hin, dass die Stilllegungsfläche in der Referenzprojektion schon fast $10 \%$ der LF erreicht. Somit besteht eine relativ geringe Flächennachfrage für die landwirtschaftliche Produktion, welche konsistent ist zum hohen Aufforstungsumfang in dieser Region.

\subsubsection{Vergleichende Beurteilung der Maßnahmen}

Die Wiedervernässung organischer Böden ist hinsichtlich der THG-Vermeidungsleistung als sehr flächen- und kosteneffizient zu bewerten. Durch den Wegfall der landwirtschaftlichen Nutzung auf den wiedervernässten Moorflächen entsteht in der Regel ein

Abb. 4.34 Regionale Änderung landwirtschaftlich genutzte Fläche in Prozentpunkten der LF unter der Maßnahme „Aufforstung“

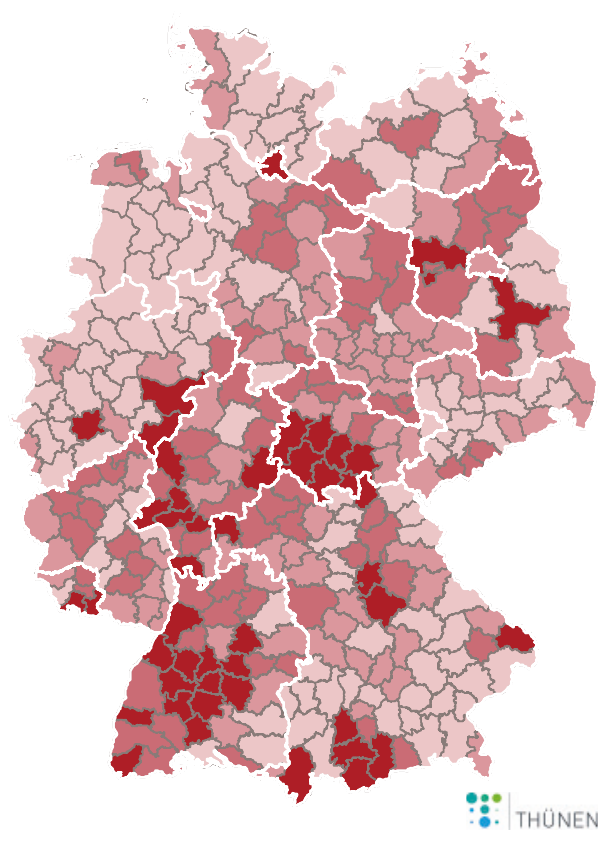

Regionale Änderung der landwirtschaftlich genutzten Fläche in \%-Punkten der LF unter der Maßnahme „Aufforstung“ (BBSRklima)

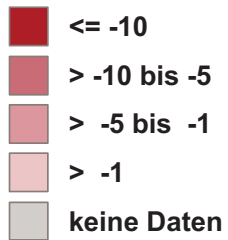

Quelle: RAUMIS-Berechnungen 2015 
ökologischer Mehrwert für Natur und Umwelt, da beispielsweise Habitate für seltene Tier- und Pflanzenarten entstehen und sich die Nutzungsaufgabe positiv auf Gewässerund Bodenschutz auswirkt. Allerdings ist die Umsetzung regional auf Standorte mit hohen Flächenanteilen landwirtschaftlich genutzter Moorböden begrenzt (z. B. in Norddeutschland) und bedeutet einen substantiellen Eingriff in das Bodeneigentum.

Die Erhebung einer Stickstoffabgabe auf den Verkaufspreis von mineralischem Stickstoff ist administrativ mit relativ geringerem Aufwand verbunden, da sie an wenigen zentralen Stellen des Handels erhoben werden könnte. Somit ist es ein Instrument, das verhältnismäßig einfach zu kontrollieren und umzusetzen ist. Allerdings muss die Abgabe zum einen hoch genug sein, um Wirksamkeit zu zeigen, und zum anderen sollte die Heterogenität der Betriebsausrichtungen berücksichtigt werden. Betriebe, welche durch Tierhaltung Zugriff auf Wirtschaftsdünger haben, setzen im Vergleich zu reinen Ackerbaubetrieben weniger mineralischen Stickstoffdünger ein. Eine Abgabe auf mineralischen Stickstoffdünger würde die Nutzung von Wirtschaftsdünger in Ackerbaubetrieben/regionen attraktiver machen.

Bei der Förderung der Energiemaisproduktion steht der hohen Flächeneffizienz eine starke Erhöhung der N-Bilanz als negative Umweltwirkung gegenüber. Des Weiteren müssen die Erhöhung des Erosionspotenzials sowie der zu erwartende Rückgang der Agrarbiodiversität beachtet werden. Außerdem sind bei unverändertem Verbrauch von Nahrungsmitteln die Emissionen durch indirekte Landnutzungsänderungen zu berücksichtigen. Neben den vergleichsweise hohen Vermeidungskosten in Höhe von rund $200 € / \mathrm{t} \mathrm{CO}_{2}$ - ̈̈qu., die auch von anderen Studien bestätigt werden (u. a. WBA 2011) sind zur Bewertung der Förderung der Energiemaisproduktion die weiteren Umweltkosten einzubeziehen.

Der Anbau von Paludikulturen stellt eine Option der Biomasseproduktion für eine energetische Nutzung auf wiedervernässten organischen Böden dar. Die Kombination von Wiedervernässung und Paludikulturen führt zu einer Extensivierung der Produktion und stellt besondere Flora- und Faunahabitate bereit und leistet neben der THGEmissionsminderung positive Beiträge zum Natur- und Umweltschutz. Die Maßnahme ist nur auf organischen Böden umsetzbar, sodass ihre Potenziale regional begrenzt sind. Nur durch die Vermeidungswirkung der Wiedervernässung organischer Böden als Voraussetzung zur Paludiproduktion erfährt die Maßnahme ein hohe Wirksamkeit und Effizienz. Die Paludikulturproduktion als separate Maßnahme wäre aufgrund der geringen Mitigationswirkung durch Substitution fossiler Energie nicht effizient.

Die obligatorische Flächenstilllegung ist mit einer deutlichen Einschränkung der landwirtschaftlichen Produktion und einem Rückgang des Einkommens verbunden. Die zu berücksichtigenden iLUC-Effekte reduzieren die Effektivität und Effizienz dieser Maßnahme. Allerdings sind positive Umweltwirkungen wie die Reduzierung der Produktionsintensität und Erhöhung der Biodiversität der Agrarlandschaft zu erwarten.

Die Aufforstung in Höhe von $10 \%$ der Waldfläche auf landwirtschaftlicher Nutzfläche führt zur regionalen Reduzierung der landwirtschaftlichen Produktion und zu einem verminderten Einsatz von Dünge- und Pflanzenschutzmitteln. Wie bei der Förderung 
der Paludiproduktion können positive Umweltwirkungen auf die Biodiversität erwartet werden.

\section{Vergleich der ökonomischen Bewertung der Maßnahmen}

Vergleicht man die Maßnahmen anhand ihrer durchschnittlichen Flächeneffizienz, kann die bereits weit überdurchschnittliche Flächeneffizienz der Wiedervernässung durch die Förderung der Paludikulturproduktion noch gesteigert werden. Die Förderung der Energiemaisproduktion steht an dritter Stelle der durchschnittlichen Flächeneffizienz (vgl. Abschn. 3.4.5).

Bezüglich der Vermeidungskosten ist die Wiedervernässung von organischen Böden als beste der untersuchten Maßnahmen zu bewerten, gefolgt von der Förderung der Paludikulturproduktion und der Aufforstung auf landwirtschaftlich genutzter Fläche. An vierter und fünfter Stelle im Ranking liegen die Förderung der Energiemaisproduktion und die Erhebung einer Stickstoffabgabe. Bei den Maßnahmen mit den geringsten Vermeidungskosten besteht der Nachteil in der regional begrenzten Anwendbarkeit. Die Maßnahmen, die auf Wiedervernässung basieren, können nur in Regionen umgesetzt werden, in denen organische Böden landwirtschaftlich genutzt werden. Auch die Aufforstung wird durch ihre langfristige Flächenumnutzung nicht auf den ertragsstarken Standorten umgesetzt werden, sondern auf den Grenzertragsstandorten. Die übrigen Maßnahmen haben den Vorteil, sich deutschlandweit umsetzen zu lassen. Dabei können der Stickstoffdüngerabgabe noch Vorteile ihrer Umweltwirkung (Reduzierung der Nitratbelastung in Gewässern) zugeschrieben werden und der Energiemaisförderung die Bereitstellung von Energie.

\subsubsection{Strategien „Landwirtschaft"}

Im Sektor Landwirtschaft werden die drei Strategien „Klimaschutz“, „Bioenergie“ sowie „Natur- und Umweltschutz“ untersucht. Die Strategie „Klimaanpassung“ wird nicht untersucht, da sich für die Landwirtschaft angesichts der kurz- bis mittelfristig erwarteten geringen Klimaänderungen und der hohen Anpassungsfähigkeit der Landwirtschaft in den nächsten 15 Jahren kein akuter Handlungsbedarf ergibt (vgl. Abschn. 3.4.5).

In den Strategien werden die beschriebenen und analysierten Einzelmaßnahmen kombiniert, da sich die aggregierte Wirkung eines Maßnahmenbündels von den jeweiligen Einzelwirkungen unterscheidet. Tab. 4.15 gibt einen Überblick über die zu Landnutzungsstrategien zusammengefassten Maßnahmen sowie deren Ausgestaltung. Dabei werden bewusst extreme Annahmen für die Umsetzung der Strategien gewählt, wobei auch negative Auswirkungen auf andere Aspekte zunächst akzeptiert werden. Die Anzahl an Maßnahmen je Strategie wird gering gehalten, um Verzerrungseffekte zu vermeiden und präzisere Aussagen zu Ursache und Wirkung treffen zu können.

RAUMIS nutzt die Flächenkulissen des Land Use Scanners als modellexogene Vorgaben für Acker- und Grünland in den drei Strategien. Die Land Use Scanner-Flächen- 
kulissen berücksichtigen die strategiespezifisch unterschiedlichen Flächenverteilungen zwischen den Sektoren Landwirtschaft, Forstwirtschaft sowie Siedlung und Verkehr $(\mathrm{SuV})$.

\subsubsection{Ergebnisse der Klimaschutzstrategie}

Die Klimaschutzstrategie folgt mit der Kombination von Instrumenten einer klimapolitischen Ausrichtung und berücksichtigt die kosteneffizienten Maßnahmen Wiedervernässung, angepasstes Düngemanagement und Aufforstung (vgl. Tab. 4.15). Hinsichtlich der Aufforstung wird ein Zuwachs der Waldfläche um $10 \%$ (1,08 Mio. ha) unterstellt, die zur Hälfte auf landwirtschaftlich genutzten Flächen (LF) erfolgt und die andere Hälfte auf nicht von landwirtschaftlichen Betrieben bewirtschafteter Landwirtschaftsfläche. Trotz der im Vergleich zur Referenzprojektion geringeren Flächeninanspruchnahme durch Siedlung und Verkehr (vgl. Tab. 4.8), steht der Landwirtschaft wegen der unterstellten Aufforstung eine um 3,1\% geringere Produktionsfläche zur Verfügung. Die Wiedervernässung ist auf maximal $30 \%$ der Moorfläche begrenzt. Mit einer Abgabe auf Mineralstickstoffdünger in Höhe von 40 \% berücksichtigt die Strategie „Klimaschutz“ ein marktbasiertes Instrument der Umweltpolitik.

Tab.4.15 Zuordnung der Maßnahmen zu den Strategien im Sektor Landwirtschaft. (Quelle: Thünen-Institut)

\begin{tabular}{|c|c|c|c|}
\hline Maßnahme & Klimaschutz & Bioenergie & $\begin{array}{l}\text { Natur- und Umwelt- } \\
\text { schutz }\end{array}$ \\
\hline $\begin{array}{l}\text { Wiedervernässung von } \\
\text { Mooren }\end{array}$ & $\begin{array}{l}\text { Max. } 30 \% \text { der Moor- } \\
\text { flächen }\end{array}$ & & $\begin{array}{l}\text { Max. } 30 \% \text { der Moor- } \\
\text { flächen }\end{array}$ \\
\hline $\begin{array}{l}\text { Anpassung Dünge- } \\
\text { management: } \\
\text { Verringerung der } \\
\text { Bewirtschaftungs- } \\
\text { intensität }\end{array}$ & $\begin{array}{l}\text { Abgabe auf } \\
\text { mineralischen Stick- } \\
\text { stoffdünger: } \\
\text { +40\% des Referenz- } \\
\text { preises in } 2030\end{array}$ & & \\
\hline Anbau Energiemais & & $\begin{array}{l}\text { Ausdehnung } \\
\text { durch zusätzliche } \\
\text { Mitigationszahlungen } \\
\text { (PES) }\end{array}$ & \\
\hline $\begin{array}{l}\text { Anbau } \\
\text { Paludikulturen nach } \\
\text { Wiedervernässung }\end{array}$ & & $\begin{array}{l}\text { Auf max. } 30 \% \\
\text { der Moorflächen; } \\
\text { Mitigationszahlungen } \\
\text { (PES) }\end{array}$ & \\
\hline $\begin{array}{l}\text { Ökologische Flächen- } \\
\text { stilllegung }\end{array}$ & & & $\begin{array}{l}\text { Flächenrestriktion } \\
\text { (Acker und Grünland): } \\
10 \% \text { der LF }\end{array}$ \\
\hline Aufforstung & $\begin{array}{l}\text { Aufforstungsgebot: } \\
+10 \% \text { Wald }\end{array}$ & & \\
\hline
\end{tabular}




\section{Sektoranalyse}

Durch die Maßnahmen reduziert sich die landwirtschaftliche Produktion. Die Stickstoffabgabe erhöht die Produktionskosten, verringert die Düngeintensität, sowohl durch eine Reduktion der speziellen Intensität als auch durch eine Anpassung der Anbaustruktur hin zu weniger düngeintensiven Kulturen, und bewirkt insgesamt eine Reduzierung des Mineralstickstoffeinsatzes. Die Kombination der Maßnahmen der Klimaschutzstrategie ergibt eine Reduktion der THG-Emissionen aus mineralischen (-2,7 Mio.t $\mathrm{CO}_{2}-$ Äqu.) und organischen Böden (-5,9 Mio.t $\mathrm{CO}_{2}$ - ̈̈qu.) sowie aus der Tierproduktion (-0,4 Mio. t $\mathrm{CO}_{2}$ - ̈̈qu.; vgl. Tab. 4.17).

Vor allem die Abgabe auf mineralischen Stickstoff wirkt sich vermindernd auf die Bewirtschaftungsintensität aus. So sinkt der Einsatz von mineralischem Stickstoff und reduziert damit die Stickstoffbilanz $(-6 \%)$. Ebenso verringert sich der Einsatz von Pflanzenschutzmitteln $(-5 \%)$. Durch die verminderte Mineralstickstoffproduktion werden in der Strategie „Klimaschutz“ etwa 1,2 Mio. t $\mathrm{CO}_{2}$-Äqu. vermieden, wenn man von einem Emissionskoeffizienten von $4 \mathrm{t} \mathrm{CO}_{2}$ - $\mathrm{Äqu}$./t industriell hergestelltem Stickstoffdünger ausgeht (Heinzlmaier 2013).

Die Emissionsvermeidungsleistung durch die $\mathrm{CO}_{2}$-Fixierung der Biomasse auf den aufgeforsteten, zuvor landwirtschaftlich genutzten Flächen wird zur Bewertung der Strategie in die THG-Bilanz aufgenommen. Die Hälfte der Aufforstungsflächen befinden sich auf der LF (vgl. Abschn. 4.5.2). Berücksichtigt man den Mittelwert der C-Sequestrierung durch die Aufforstung auf der LF der Jahre 2015 bis 2030 bei der Berechnung der Netto-THG-Emissionen der Klimastrategie, inklusive der Vermeidung infolge der reduzierten Mineralstickstoffdüngerherstellung, verringern sich diese um 15,3 Mio. t $\mathrm{CO}_{2}$ - ̈̈qu. (exklusiv iLUC-Effekte) und um 11,8 Mio. $\mathrm{C} \mathrm{CO}_{2}$ - $\ddot{A q u}$. (inklusiv iLUC-Effekte; vgl. Tab. 4.18). Ein Simulationszeitraum über das Jahr 2030 hinaus würde den C-Speicher im Wald deutlich erhöhen, da der Wald in dieser Phase den höchsten jährlichen Biomassezuwachs aufweist.

Die Stilllegungsflächen nehmen zu (+38 \%), was vor allem daran liegt, dass die Stickstoffabgabe die Pflanzenproduktion verteuert und dadurch reduziert.

Die Förderung der Wiedervernässung reduziert die meisten Pflanzenproduktionsverfahren um insgesamt 0,27 Mio. ha wiedervernässter Moorfläche (Tab. 4.16). Die Silomaisfläche steigt, um den Verlust der Futterflächen auf organischen Böden zu kompensieren.

\section{Regionalanalyse}

Die regionale Betrachtung der Klimaschutzstrategie zeigt, dass die THG-Emissionen insbesondere in den Moorregionen infolge der Wiedervernässung reduziert werden (Abb. 4.35a). Demgegenüber betreffen die Einkommenseinbußen durch die Stickstoffabgabe deutschlandweit insbesondere die Regionen mit intensiven Ackerbau und geringer Viehdichte (z. B. Mitteldeutschland; Abb. 4.35b). In Regionen mit intensiver Ackerbauproduktion und hohem Viehbesatz fallen die produktionsbedingten Einkommensverluste geringer aus (z. B. in Nordrhein-Westfalen), da der reduzierte Mineralstickstoffdünger- 


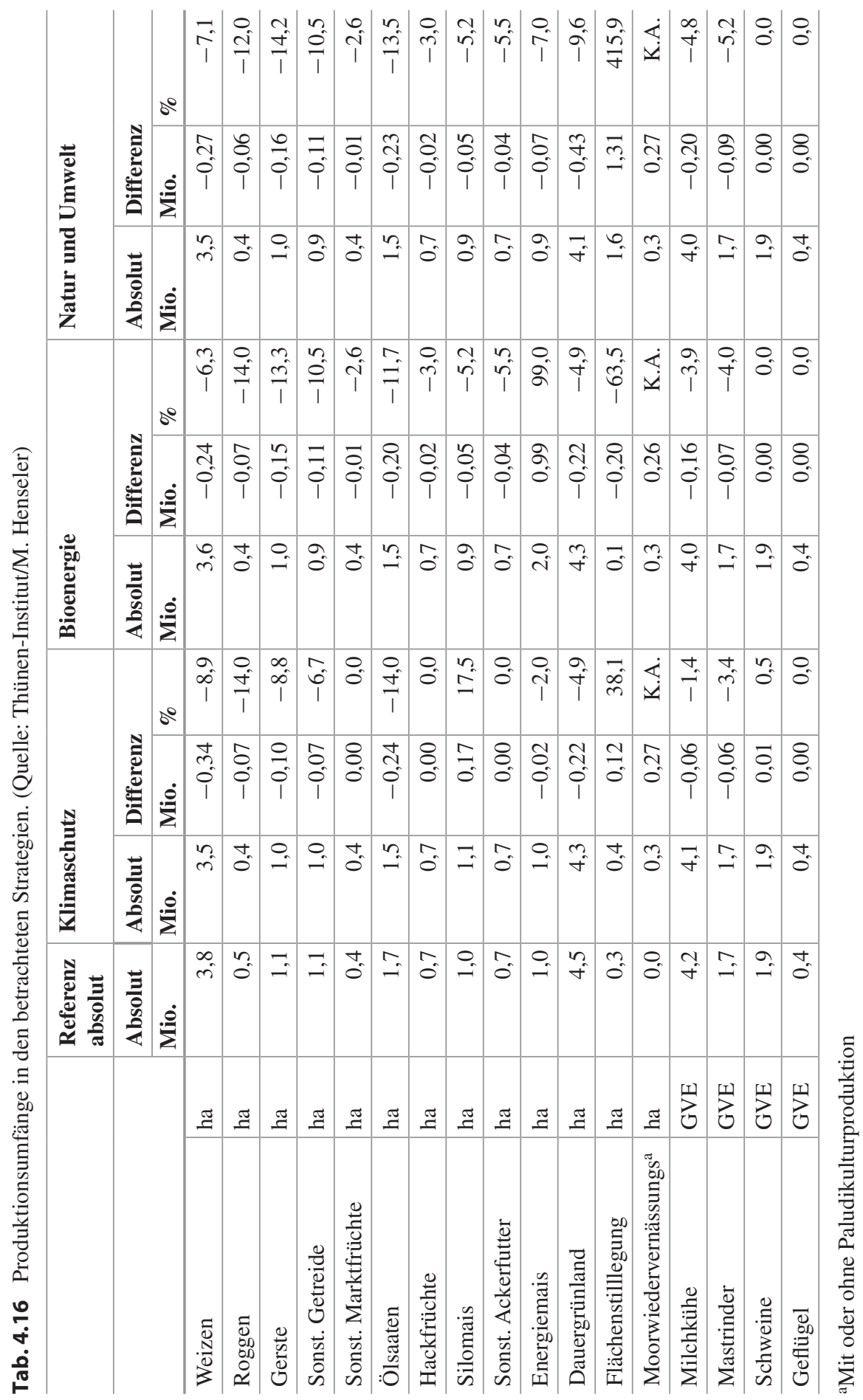


a

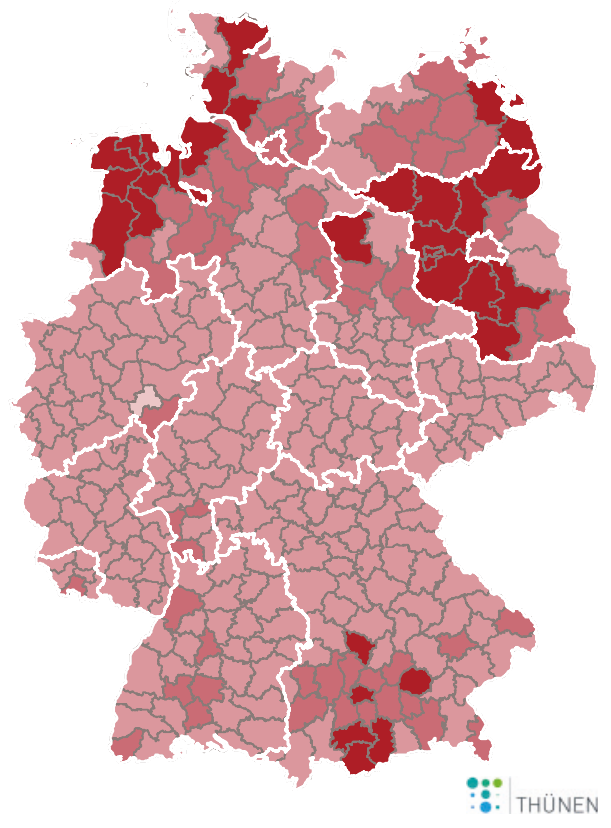

Regionale Änderung der Netto THG-Emissionen in $\mathrm{t} \mathrm{CO}_{2}$-Äquivalent ha-1 unter der Strategie „Klimaschutz“

\footnotetext{
$<=-1,0$

$>-1,0$ bis $-0,5$

$>-0,5$ bis 0

$\square>0$

keine Daten
}

Quelle: RAUMIS-Berechnungen 2015 b

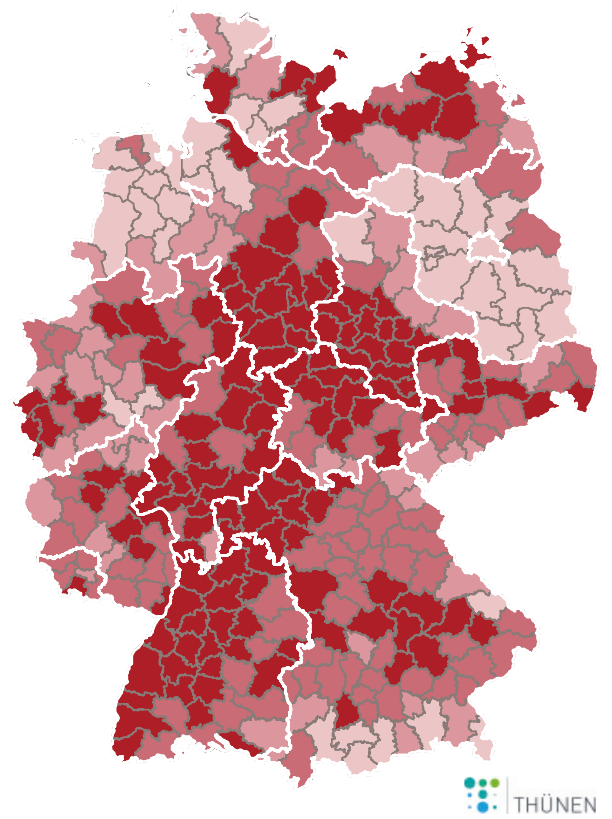

Regionale Änderu
landwirtschaftlich
inklusive Mitigation
EUR ha hnter de $^{-1}$ unte
"Klimaschutz"
$<=-200$
$>-200$ bis -150
$>-150$ bis -100
$>-100$
$\square$ keine Daten

Quelle: RAUMIS-Berechnungen 2015

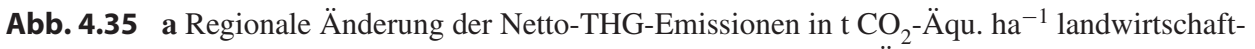
lich genutzter Fläche unter der Strategie „Klimaschutz“, b Regionale Änderung des landwirtschaftlichen Einkommens inklusive Mitigationszahlungen in EUR ha ${ }^{-1}$ landwirtschaftlich genutzter Fläche unter der Strategie „Klimaschutz“

einsatz mit organischer Stickstoffdüngung aus der Tierhaltung kompensiert werden kann. In Regionen mit hohen Anteilen an Moorflächen trägt die geringe Profitabilität der Paludikulturen zu den Einkommensverlusten bei (z. B. Norddeutschland). 


\subsubsection{Ergebnisse der Bioenergiestrategie}

Die Bioenergiestrategie setzt den Schwerpunkt auf THG-Emissionsvermeidung durch die Substitution fossiler Energieträger durch Bioenergie und enthält die Einzelmaßnahmen „Energiemaisanbau für die Biogasproduktion“ sowie den „Anbau von Paludikulturen auf wiedervernässten Moorflächen“ (vgl. Tab. 4.15). Durch die Siedlungs- und Verkehrspolitik wird weniger landwirtschaftliche Nutzfläche umgewandelt als in der Referenzprojektion. Der verringerte Flächenverlust durch die Siedlungs- und Verkehrspolitik bedeutet eine geringere Reduzierung der Grünlandfläche und kann daher auch als eine Erhaltung des Grünlands interpretiert werden (vgl. Abschn. 4.5.2). Ebenso bleiben Ackerflächen durch die Nichtumwandlung erhalten. Dadurch steht für eine gesteigerte Produktion von Biomasse zur energetischen Verwendung mehr Ackerfläche bereit als in der Referenzprojektion. Die landwirtschaftlichen Produktionsaktivitäten werden um die Produktion von extensiven Paludikulturen auf An- und Niedermoorstandorten ergänzt.

\section{Sektoranalyse}

In der Bioenergiestrategie bewirkt die Substitution fossiler Energien durch Energiemais und Paludikulturen eine Senkung der Gesamt-THG-Emissionen um 18,1 Mio. t $\mathrm{CO}_{2}$ Äqu. (exkl. iLUC-Effekte) und um 14,5 Mio. t $\mathrm{CO}_{2}$-Äqu. (inkl. iLUC-Effekte; vgl. Tab. 4.18). Davon entfallen ca. 5,7 Mio. t $\mathrm{CO}_{2}$ - ̈̈qu. auf die Wiedervernässung, die eine Voraussetzung der Paludikulturproduktion ist, und daher einen indirekten Vermeidungseffekt darstellt. Die energetische Nutzung der Paludikulturproduktion trägt nur 0,8 Mio. t $\mathrm{CO}_{2}$ - ̈̈qu. zum Vermeidungseffekt bei und ist somit deutlich geringer als die Vermeidung durch Energiemais mit 9,9 Mio. t $\mathrm{CO}_{2}$-Äqu. Ohne Berücksichtigung der Vermeidungseffekte durch Wiedervernässung organischer Böden betragen die Vermeidungseffekte der Strategie 12,4 Mio. t $\mathrm{CO}_{2}$-Äqu. (exkl. iLUC-Effekte) und 8,8 Mio. t $\mathrm{CO}_{2}$-Äqu. (inkl. iLUC-Effekte).

Die Maßnahmen bewirken eine deutliche Verschiebung von der Nahrungsmittel- zur Biomasseproduktion, wodurch auch die Tierhaltung - insbesondere die Rinderhaltung rückläufig ist. Dementsprechend gehen die Emissionen aus der Tierhaltung um 0,9 Mio. t $\mathrm{CO}_{2}$-Äqu. zurück (vgl. Tab. 4.17). Während Paludikulturen ein extensives Produktionsverfahren darstellen, ist der Anbau von Energiemais eher zu den düngeintensiven Verfahren zu zählen. Darüber hinaus fallen bei der Vergärung des Energiemaises Gärreste an, die als Wirtschaftsdünger dem Pflanzenbau zugeführt werden. Da der Wirtschaftsdünger im Vergleich zum mineralischen Dünger eine geringere Düngeeffizienz aufweist, treten höhere Stickstoffbilanzüberschüsse im Vergleich zum substituierten Pflanzenbauverfahren auf, sodass neben den positiven Wirkungen der Strategie hinsichtlich der THG-Einsparung auch negative Wirkungen zu Lasten der Umwelt erfolgen, wie am Stickstoffbilanzsaldo deutlich wird (+5\%, Tab. 4.17).

Die Energiemaisfläche wird um das Doppelte ausgedehnt (+99\%), was einen Rückgang der Stilllegungsflächen $(-64 \%)$, des Dauergrünlands $(-5 \%)$ sowie aller Ackerkulturen zur Folge hat. Der Paludikulturanbau nimmt 0,26 Mio. ha auf wiedervernässten 
Tab. 4.17 Auswirkungen der betrachteten Strategien auf das landwirtschaftliche Einkommen und die Umwelt in Deutschland. (Quelle: Thünen-Institut/M. Henseler)

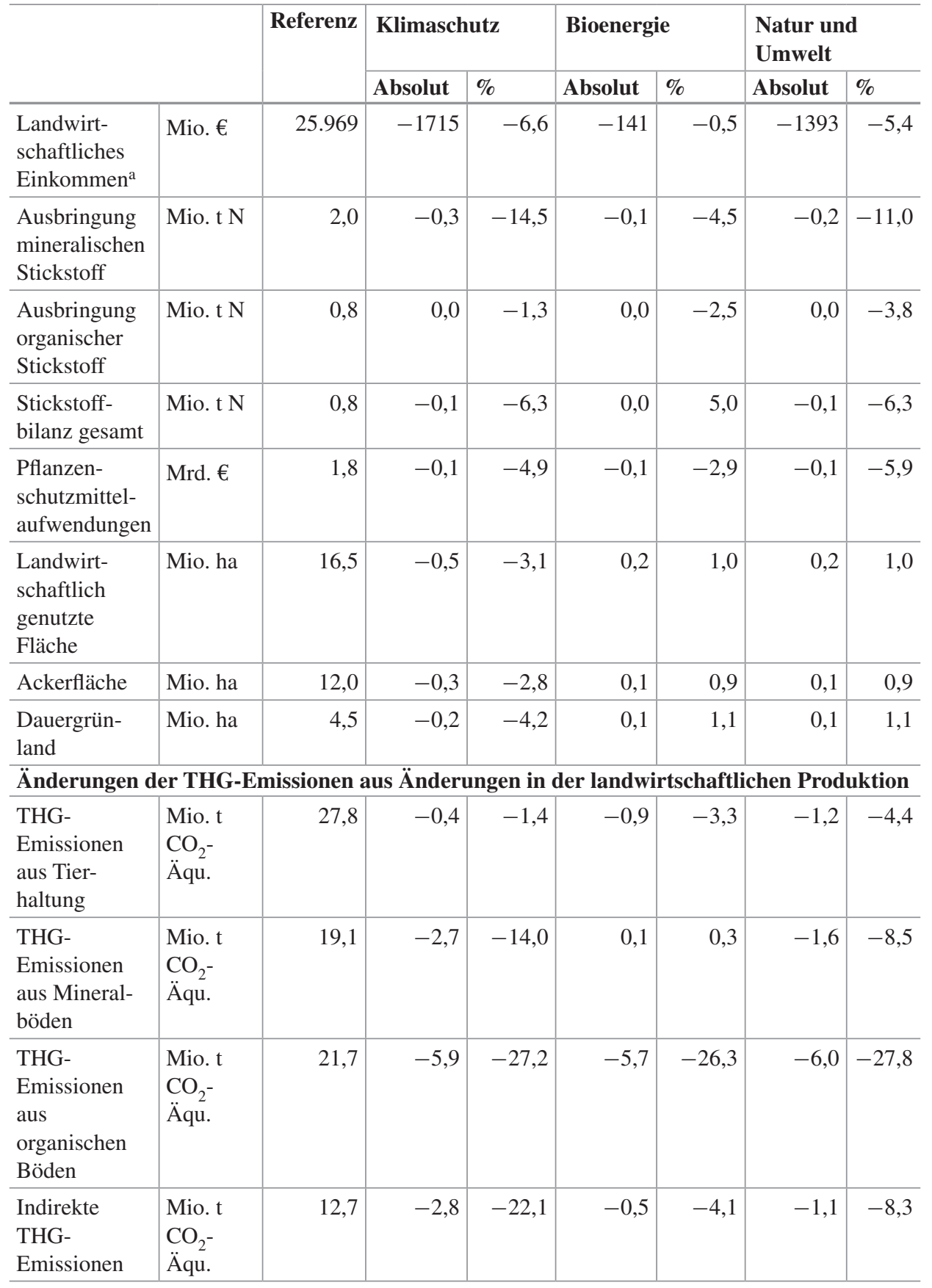

(Fortsetzung) 
Tab.4.17 (Fortsetzung)

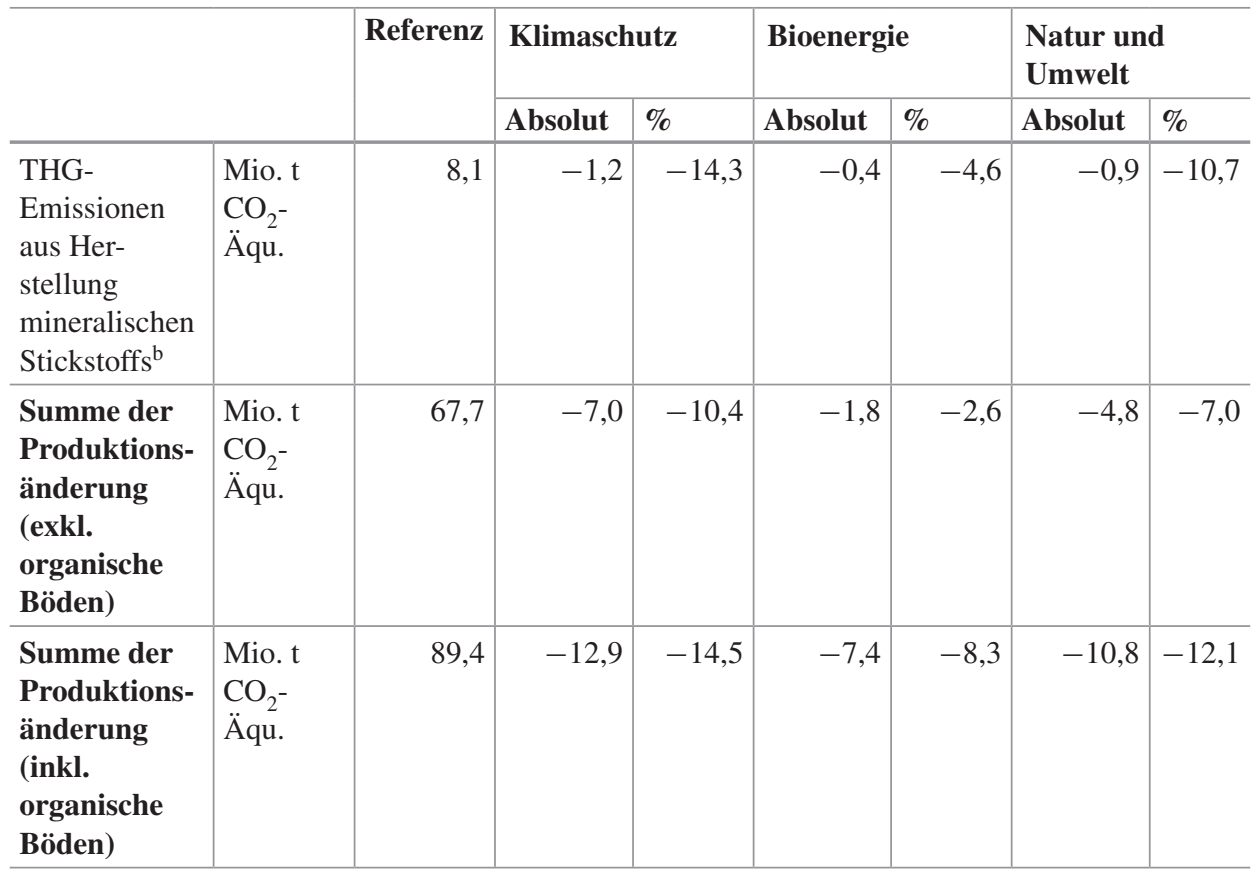

${ }^{\mathrm{a} A l s}$ Nettowertschöpfung zu Faktorpreisen (NWSF)

${ }^{b}$ Emissionsfaktor von 4 t $\mathrm{CO}_{2}$ - $\mathrm{Äqu}$./t N

An- und Niedermooren ein. Durch den Rückgang der Nahrungsmittelproduktion sinkt das landwirtschaftliche Einkommen geringfügig $(-1 \%)$.

\section{Regionalanalyse}

Der THG-Bilanzsaldo reduziert sich deutschlandweit und vor allem in den moorreichen Regionen durch den indirekten Vermeidungseffekt der Wiedervernässung in der Paludikulturproduktion. Weiter nimmt der Maisanbau für Biogas in den bereits in der Referenzprojektion durch Energiemais geprägten Regionen stark zu, wodurch dort hohe Vermeidungsleistungen zu verzeichnen sind. Die größten Vermeidungseffekte sind v. a. in Norddeutschland sowie in einigen Regionen Süddeutschlands zu beobachten (Abb. 4.36a).

Das landwirtschaftliche Einkommen ändert sich in weiten Teilen Deutschlands nur geringfügig. Im Norden und im Süden Deutschlands bewirkt der wenig rentable Paludianbau die Verluste in landwirtschaftlichen Einkommen. Auf den Ackerbaustandorten hingegen in Mittel- und Süddeutschland entstehen durch die Ausdehnung der Energiemaisproduktion geringe Gewinne (Abb. 4.36b). 


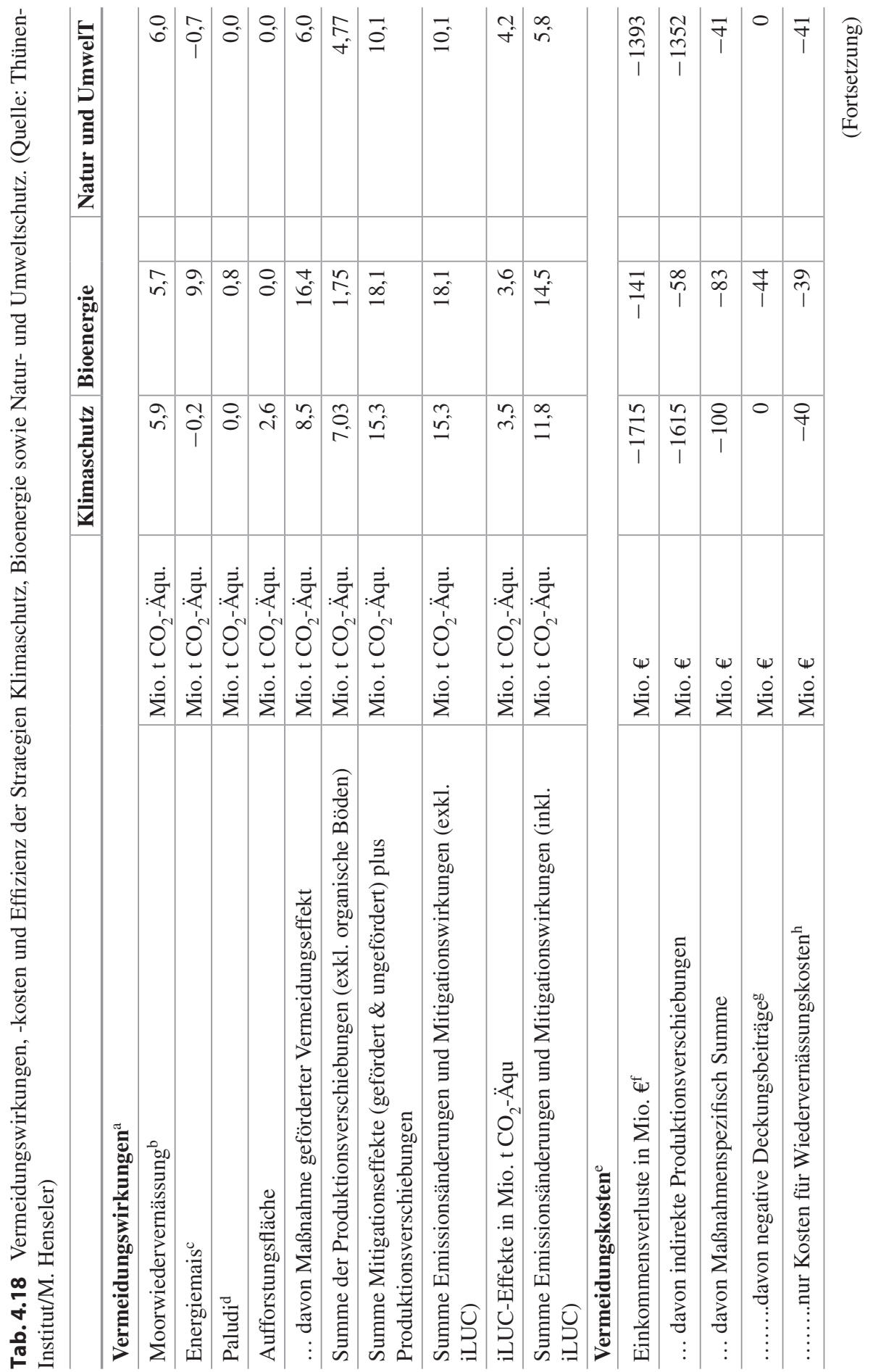




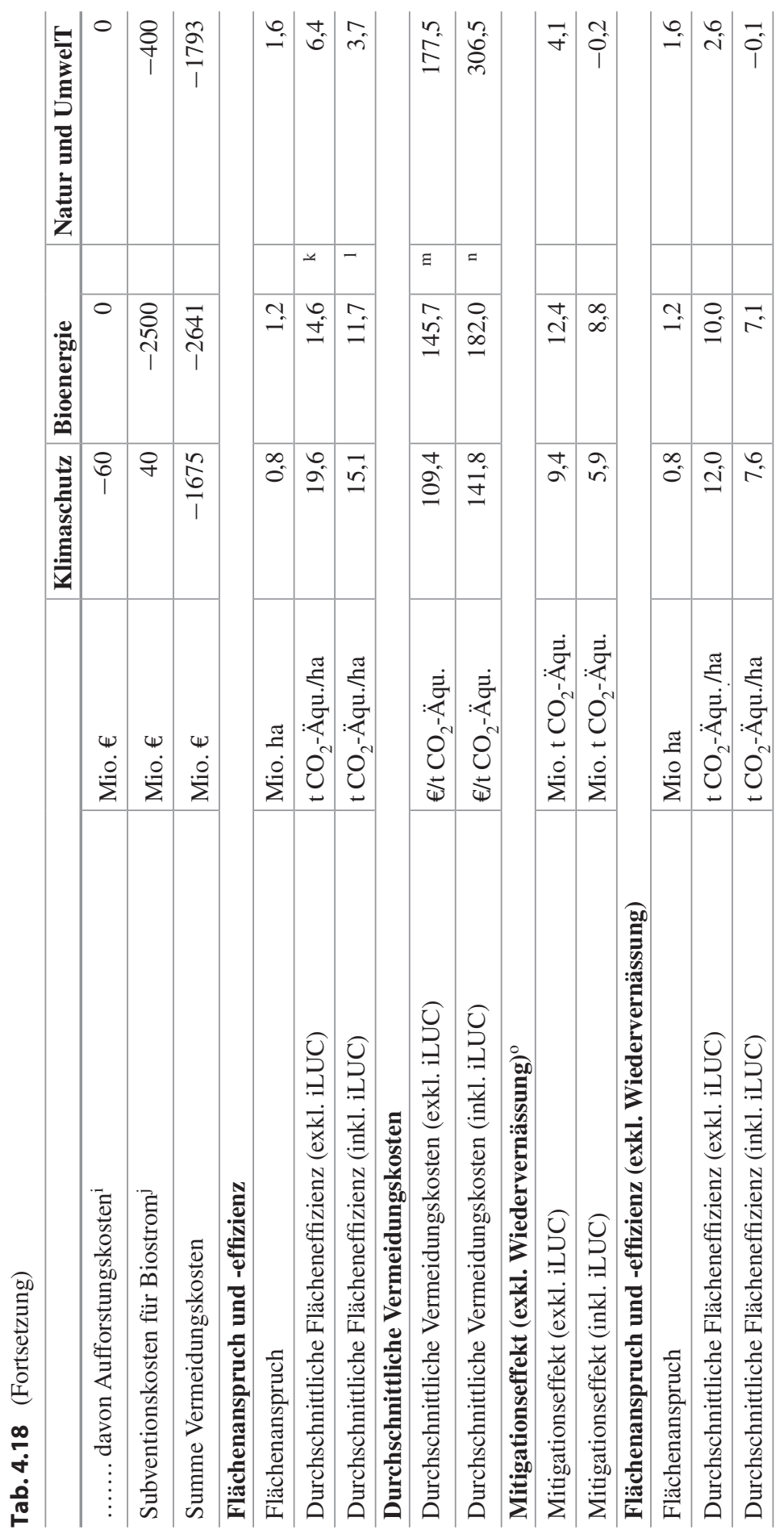




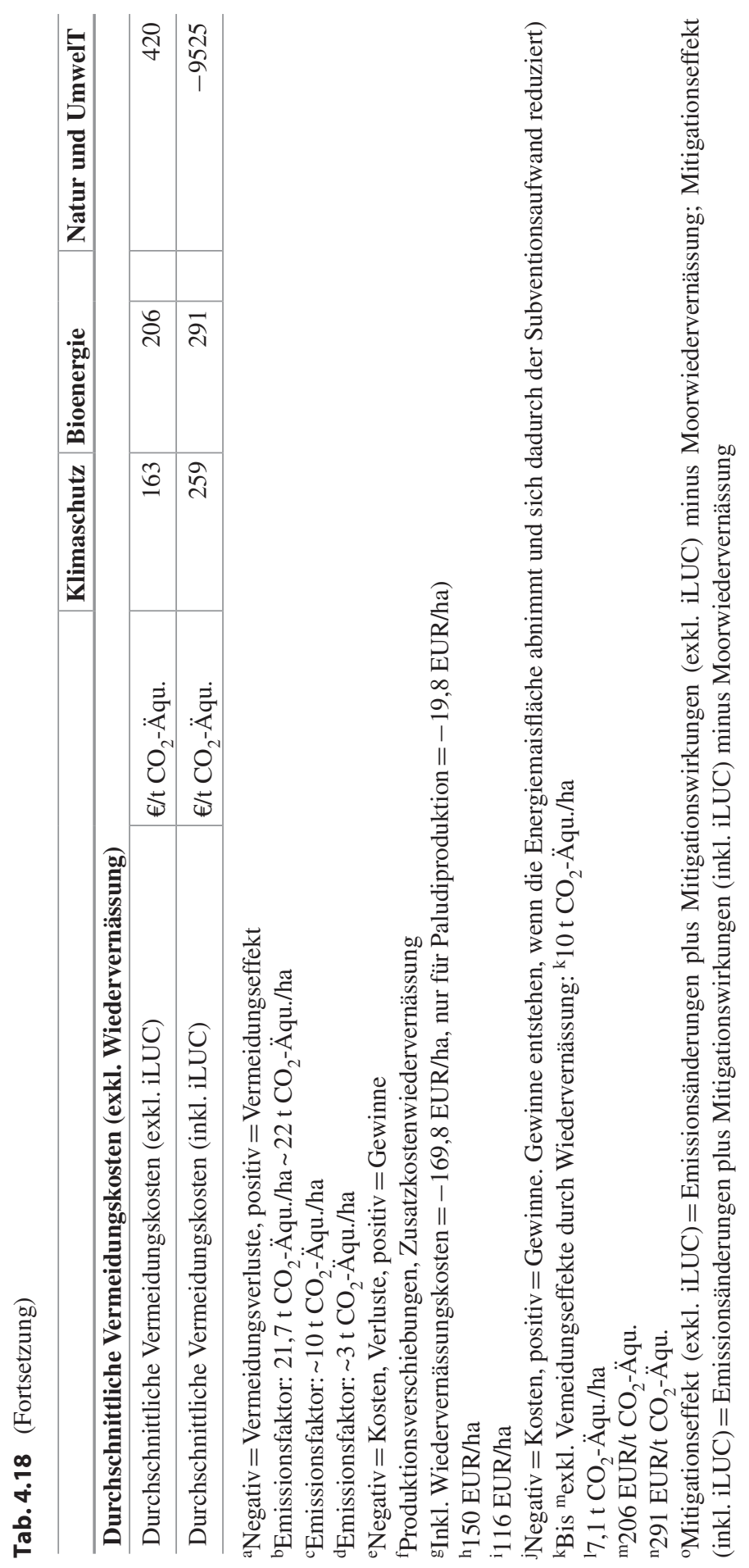


(a)

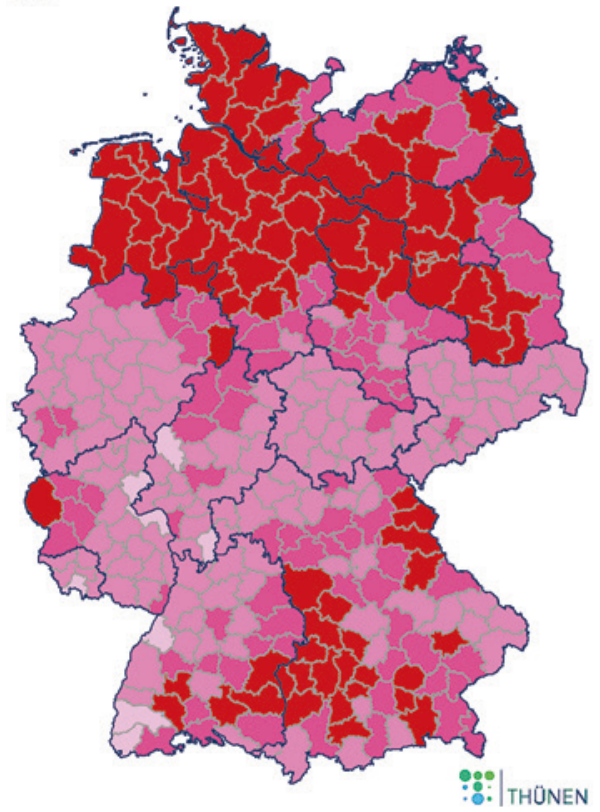

(b)

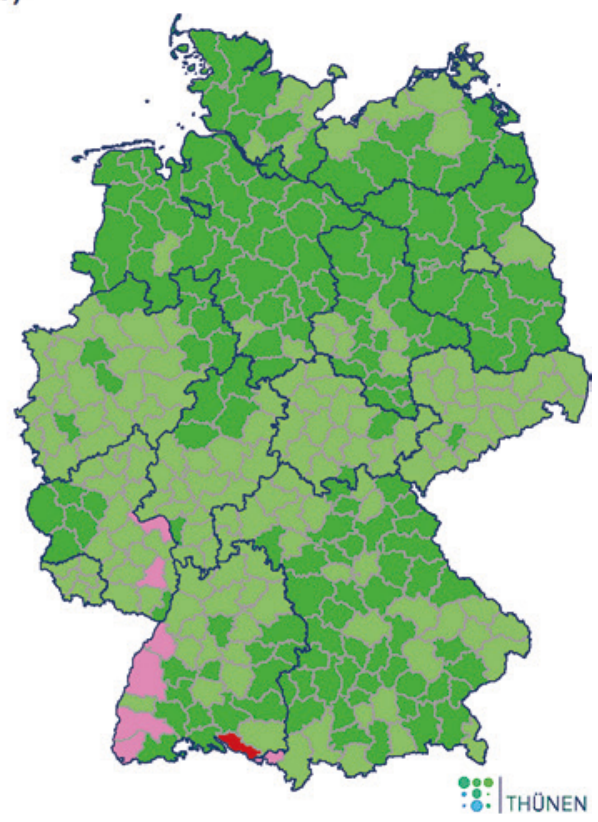

Regionale Änderung des landwirtschaftlichen Einkommens inklusive Mitigationszahlungen in EUR pro Hektar landwirtschaftlich genutzte Fläche unter der Strategie „Energie durch Biomasse“

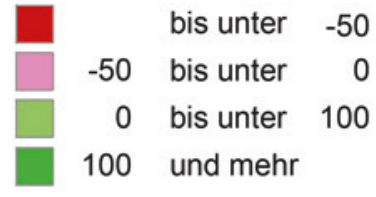

Quelle: RAUMIS-Berechnungen 2015

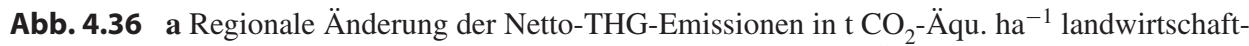
lich genutzte Fläche unter der Strategie „Bioenergie“, b Regionale Änderung des landwirtschaftlichen Einkommens inklusive Mitigationszahlungen in EUR ha ${ }^{-1}$ landwirtschaftlich genutzte Fläche unter der Strategie „Bioenergie“

\subsubsection{Ergebnisse der Natur- und Umweltschutzstrategie}

Die Natur- und Umweltschutzstrategie setzt den Schwerpunkt auf THGEmissionsvermeidung durch Maßnahmen mit weiteren positiven Wirkungen auf Natur und Umwelt. Durch eine entsprechende Siedlungs- und Verkehrspolitik wird in der Naturund Umweltschutzstrategie wie in der Bioenergiestrategie weniger landwirtschaftliche Nutzfläche umgewandelt als in der Referenzprojektion. Dadurch bleibt mehr Fläche in 
landwirtschaftlicher Produktion. Verglichen mit der Nutzung von Siedlungs- und Verkehrsflächen hat die landwirtschaftliche Nutzfläche einen ökologisch höheren Wert. Der verringerte Flächenverlust durch Siedlung und Verkehr bedeutet einen geringeren Rückgang der Grünlandfläche und kann daher auch als eine Erhaltung des Grünlands interpretiert werden (vgl. Abschn. 4.5.2).

Wie in der Klimaschutzstrategie wird das Instrument der THG-Vermeidungszahlungen auf die vermiedenen THG-Emissionen aus wiedervernässten Moorböden (maximal $30 \%$ der Moorfläche) angewendet. Die Stilllegungsfläche wird als verpflichtende ökologische Vorrangfläche für $10 \%$ der LF vorgeschrieben und kann sich auf Acker- und/oder auf Grünlandfläche beziehen. Dadurch wird bei der Modellierung eine größere Flexibilität angenommen, als es für die Implementierung der ökologischen Vorrangflächen im Rahmen der GAP der Fall ist, wo $5 \%$ der Ackerfläche gemeldet werden müssen.

\section{Sektoranalyse}

In der Natur- und Umweltschutzstrategie erhöht die verpflichtende Flächenstilllegung den Anteil von stillgelegtem Acker- und Grünland. Die stillgelegten oder die wiedervernässten Flächen werden nicht mehr zur Nahrungsmittel- oder Energie- und Futterproduktion genutzt. Die wiedervernässten Moorflächen betragen 0,27 Mio. ha; die zusätzliche Stilllegungsfläche beträgt 1,31 Mio. ha (vgl. Tab. 4.16) und ergibt einen Stilllegungsanteil von annahmegemäß $10 \%$ der LF.

Die Produktionseinschränkung senkt die Emissionen aus der Tierhaltung ( $-1,2$ Mio. $\mathrm{t}$ $\mathrm{CO}_{2}$ - Äqu.) und aus Mineralböden (-1,6 Mio. $\mathrm{t} \mathrm{CO}_{2}$ - ̈̈qu.; vgl. Tab. 4.17). Die Kombination dieser Maßnahmen mit der Förderung von Wiedervernässung organischer Böden reduziert die THG-Emissionen um insgesamt 10,1 Mio. t $\mathrm{CO}_{2}$ - ̈̈qu. (exkl. iLUCEffekte) und um 5,8 Mio. t $\mathrm{CO}_{2}$-Äqu. (inkl. iLUC-Effekte; vgl. Abb. 4.37; Tab. 4.18).

Der damit verbundene Rückgang der landwirtschaftlichen Produktion führt neben der Reduzierung der THG-Emissionen zur Senkung des Stickstoffbilanzsaldos (-6\%, vgl. Tab. 4.17) sowie des Pflanzenschutzmitteleinsatzes (-6\%). Dadurch werden mit der Natur- und Umweltschutzstrategie die angestrebten positiven Umweltwirkungen auf Wasserbelastung und Biodiversität erreicht. Die Einbußen des landwirtschaftlichen Einkommens betragen $-5 \%$.

\section{Regionalanalyse}

Eine Minderung der THG-Emissionen findet in der Natur- und Umweltschutzstrategie deutschlandweit statt, aber insbesondere in den Moorregionen infolge der Wiedervernässung (Abb. 4.37a).

Regionen mit hohen Einkommenseinbußen finden sich in Nord- und Süddeutschland (Abb. 4.37b). In diesen Gunstregionen sind die Anteile der Stilllegungsfläche in der Referenzprojektion besonders niedrig (z. B. im sehr intensiv bewirtschafteten SchleswigHolstein). Die verpflichtende Flächenstilllegung bedeutet hier einen relativ hohen Verlust 
a

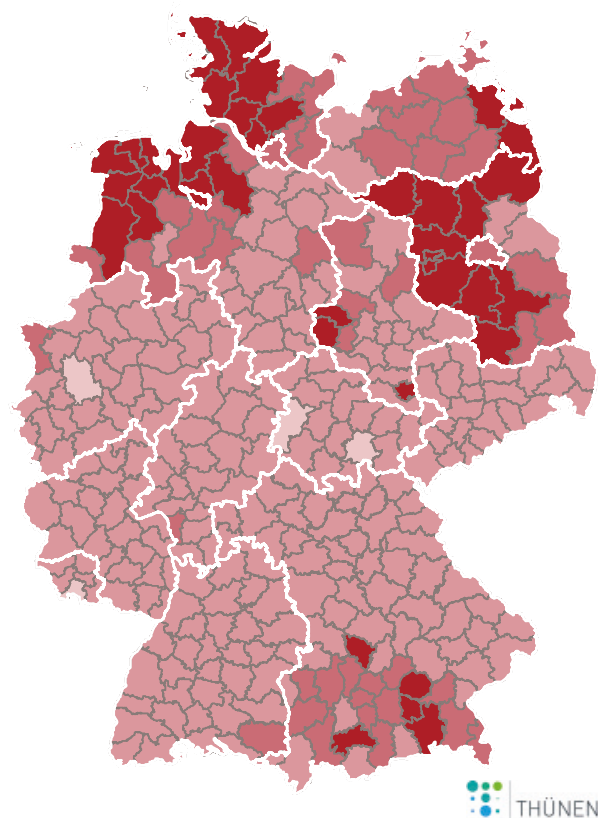

Regionale Änderung der Netto-THGEmissionen in $\mathrm{t} \mathrm{CO}_{2}$-Äquivalent ha- ${ }^{-1}$ unter der Strategie „Natur- und Umweltschutz"

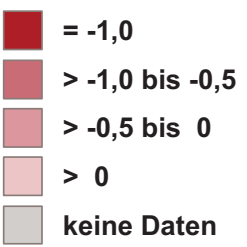

Quelle: RAUMIS-Berechnungen 2015 b

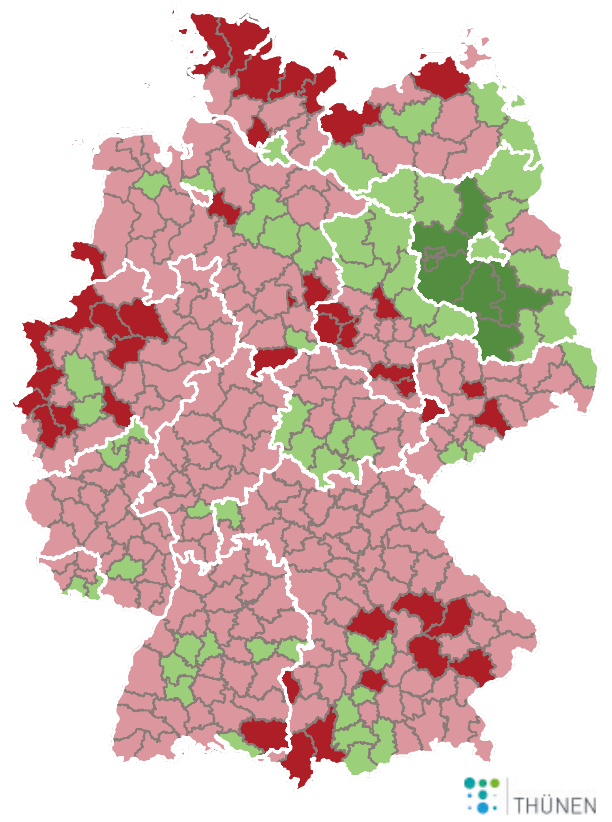

Regionale Änderung des landwirtschaftlichen Einkommens inklusive Mitigationszahlungen in EUR ha-1 unter der Strategie „Naturund Umweltschutz"

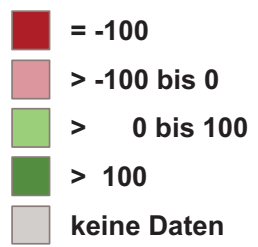

Quelle: RAUMIS-Berechnungen 2015

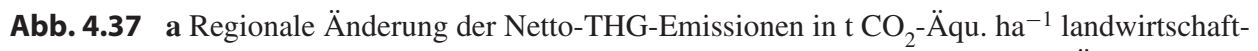
lich genutzte Fläche unter der Strategie „Natur- und Umweltschutz“, b Regionale Änderung des landwirtschaftlichen Einkommens inklusive Mitigationszahlungen in EUR ha ${ }^{-1}$ landwirtschaftlich genutzte Fläche unter der Strategie „Natur- und Umweltschutz“

an Produktionsfläche von bis zu $10 \%$ und führt zu entsprechenden Einkommensverlusten. Dahingegen sind die Zunahme an Flächenstilllegung und die Einkommensverluste in Regionen geringer, wo es in der Referenzprojektion bereits höhere Anteile an Flächenstilllegungsflächen gibt (z. B. in Ostdeutschland). 


\subsubsection{Vergleichende Beurteilung der landwirtschaftlichen Strategien}

\section{Vor- und Nachteile der Strategien}

Der Vergleich der simulierten Strategien zeigt unterschiedliche Wirkungen bezüglich der verschiedenen Ziele und Indikatoren. Alle drei Strategien wirken sich positiv auf THGEmissionen und Mitigationsleistung aus. Die Vermeidungseffekte entstehen durch unterschiedliche Maßnahmenkombinationen, welche sich ihrerseits unterschiedlich auf das landwirtschaftliche Einkommen und die Umwelt auswirken. Die Klimaschutzstrategie kombiniert Maßnahmen, welche zum einen die Emissionen aus den größten flächenbasierten Emissionsquellen reduzieren (Mineralböden, organische Böden), und zum anderen die C-Sequestrierung (Aufforstung auf LF). Dabei werden den Maßnahmen auch positive Umweltwirkungen zugeordnet - wie die Reduzierung von Nitrateinträgen in die Gewässer und Erhöhung der Biodiversität.

Die Anwendung der flächenbasierten Maßnahme ,Wiedervernässung und Aufforstung " ist regional begrenzt, wodurch die deutschlandweit umsetzbare Maßnahme der Stickstoffabgabe eine kompensierende Funktion erfährt. Die Stickstoffabgabe bewirkt dabei nicht nur eine Senkung der Mineraldüngung und der Emission aus Mineralböden, sondern auch eine Vermeidung von Emissionen, die bei der industriellen Mineraldüngerproduktion entstehen. Durch Flächenverluste, Senken der Produktionsintensität und Kosten für die Maßnahmenumsetzung entstehen der Landwirtschaft relative hohe Einkommensverluste.

In der Bioenergiestrategie geht die große Vermeidungswirkung auf die intensive Produktion von Energiemais zurück, wodurch fossile Energieträger substituiert werden. Diese Vermeidungsleistung geht mit einer negativen Umweltwirkung einher, nämlich der Erhöhung des Stickstoffbilanzsaldos, welche das Risiko der Grundwasser- und Oberflächengewässerbelastung durch Nitrateinträge erhöht. Somit wird in der Bioenergiestrategie die höhere Vermeidungsleistung auf Kosten einer Verschlechterung anderer Umweltindikatoren erzielt. Der Produktion von Paludikulturen kommt eine geringere Bedeutung zu. Die größte Vermeidungswirkung der Maßnahme generiert die ,vorgeschaltete" notwendige Wiedervernässung organischer Böden. Die Paludiproduktion trägt zu positiven Umweltwirkungen bei, ist aber regional begrenzt. Mit der Bioenergieproduktion wird eine zusätzliche Einkommensalternative gefördert, wodurch Einkommensverluste in der Landwirtschaft sehr gering ausfallen.

In der Strategie „Natur- und Umweltschutz“ werden flächenbasierte Extensivierungsmaßnahmen kombiniert. Die Wiedervernässung organischer Böden wird als regional begrenzte Mitigationsmaßnahme gefördert. Die verpflichtende Flächenstilllegung bewirkt die deutschlandweite Reduktion der Produktionsintensität und der Emissionen aus der landwirtschaftlichen Produktion. Beiden Maßnahmen werden positive Umweltwirkungen zugeschrieben. Die Einschränkung der Produktionsfläche führt zu relativ hohen Einkommensverlusten. 
In allen drei Strategien bewirken die Änderungen der landwirtschaftlichen Landnutzung in Deutschland indirekte Landnutzungsänderungen (iLUC) in anderen Teilen der Welt. Sinkt in Deutschland die Nahrungsmittelproduktion, wird das Defizit durch Importe ausgeglichen, was zu Intensivierungen und Rodungen in anderen Ländern führen kann. Die dadurch entstehenden THG-Emissionen werden über Schätzwerte nach Osterburg et al. (2013) berücksichtigt. Die iLUC-Effekte fallen besonders hoch aus in der Naturund Umweltschutzstrategie, gefolgt von der Bioenergiestrategie und der Klimaschutzstrategie. Durch die Berücksichtigung der iLUC-Effekte verschlechtern sich v. a. die Vermeidungseffekte der Natur- und Umweltschutzstrategie (vgl. Tab. 4.18).

Um die Gesamtwirkung der Strategien einschätzen zu können, müssen THGEmissionen berücksichtigt werden, die anderen Sektoren als der Landwirtschaft zugerechnet werden.

\section{Vergleichende ökonomische Bewertung der Strategien}

In Tab. 4.18 werden Simulationsergebnisse für die Strategien zusammengestellt mit ihren Vermeidungskosten - differenziert nach Kostenpositionen, Emissionsquellen, Durchschnittskosten und aggregierten Durchschnittskosten - sowie die Vermeidungseffizienz bezogen auf die für die Maßnahme relevante Bezugsfläche. Diese Gegenüberstellung ermöglicht einen ökonomischen Vergleich der Strategien über das Merkmal „Kosten“.

Die vergleichsweise niedrigen Mitigationszahlungen in Kombination mit den relativ hohen Vermeidungsleistungen (aus landwirtschaftlicher Produktion, der Mineraldüngerproduktion, aus der Aufforstung) und geringeren iLUC-Effekten lassen die Klimaschutzstrategie als die kosten- und flächeneffizienteste Strategie dastehen. Gegenüber den anderen Strategien erweist sich die Klimaschutzstrategie als die beste hinsichtlich der durchschnittlichen Vermeidungskosten bedingt durch die Kombination verschiedener Politikinstrumente (Vermeidungszahlungen, Stickstoffabgabe, Aufforstung). Die beiden anderen Strategien zielen mit den Politikinstrumenten auf die spezifischen Vermeidungsaspekte der Energieproduktion und des Natur- und Umweltschutzes, welche negative Auswirkungen auf Umwelt oder Einkommensaspekte haben.

Vergleicht man die Strategien anhand ihrer durchschnittlichen Flächeneffizienz, ist die Klimaschutzstrategie die beste Strategie gefolgt von der Bioenergiestrategie (vgl. Tab. 4.18). Auch bezüglich der Vermeidungskosten schneidet die Klimaschutzstrategie am besten $a b$. Für die Bioenergiestrategie hängt die Höhe der durchschnittlichen Vermeidungskosten davon $\mathrm{ab}$, ob die Vermeidungseffekte durch die Wiedervernässung berücksichtigt werden oder nicht.

Werden die Vermeidungseffekte durch Wiedervernässung als indirekte Effekte betrachtet und als Voraussetzung zur Paludiproduktion der Strategie zugeschrieben, liegen die durchschnittlichen Vermeidungskosten zwischen denen der beiden anderen Strategien. Werden die iLUC-Effekte nicht berücksichtigt und die Vermeidungseffekte durch Wiedervernässung nicht der Bioenergiestrategie zugerechnet, fallen die durchschnittlichen Vermeidungskosten der Bioenergiestrategie (206 EUR/t $\mathrm{CO}_{2}$ Äqu.) höher aus als die Vermeidungskosten der Strategien „Natur- und Umweltschutz“ 
(178 EUR/t CO $\mathrm{CO}_{2}$ - ̈̈qu.) oder „Klimaschutz“ (177 EUR/t $\mathrm{CO}_{2}$-Äqu.). Unter Berücksichtigung der iLUC-Effekte liegen die durchschnittlichen Vermeidungskosten der Bioenergiestrategie (291 EUR/t CO $\mathrm{CO}_{2}$ Äqu.) zwischen denen der Strategien „Natur- und Umweltschutz“ (307 EUR/t CO 2 -Äqu.) und „Klimaschutz“ (142 EUR/t CO 2 -Äqu.).

\section{Akzeptanz der Strategien}

Die Ergebnisse zeigen, dass die untersuchten Strategien neben ihren unterschiedlichen THG-Emissionsvermeidungswirkungen verschiedene relative Vorzüglichkeiten für Vertreter der Produktion, der Politik und des Umweltschutzes aufweisen (vgl. Tab. 4.19).

Die Klimaschutzstrategie beinhaltet eine Stickstoffabgabe, welche die Produktionskosten für die Landwirtschaft erhöht. Eine Stickstoffabgabe wird von der Landwirtschaft auch dann nur bedingt auf Akzeptanz stoßen, wenn die vereinnahmten Mittel der Stickstoffabgabe rückerstattet werden und die Verluste zu einem Großteil kompensieren. Die Stickstoffabgabe bedeutet für die mineralstickstoffproduzierende Industrie Absatzeinbußen. Für den Staat ist die Stickstoffabgabe ein effektives Instrument, welches allerdings eine Überwachung der Stickstoffströme entlang der gesamten Wertschöpfung erfordert. Auf Seiten der Vertreter des Umweltschutzes ist mit der Akzeptanz für die Stickstoffabgabe mit ihrer stickstoffreduzierenden Wirkung sowie, unter der Voraussetzung der Wahl geeigneter Flächen, für die Wiedervernässung zu rechnen. Die C-Speicherung der Aufforstungsflächen wird hier auch auf positive Resonanz stoßen. Zahlungen für die Wiedervernässung können lediglich Landwirte bekommen, die über wiedervernässbare, organische Böden verfügen - für diese wäre die Wiedervernässung ein tiefgreifender Eingriff in das Bodeneigentum.

Die Bioenergiestrategie ermöglicht eine Einkommenssteigerung für die Landwirtschaft und wird daher große Akzeptanz bei den Produzenten finden. Für den Staat bedeuten die Förderung der Bioenergieproduktion erhöhte Ausgaben für ein Folgeregime des EEG sowie zusätzliche Mitigationszahlungen, welche auf den Konsumenten übertragen werden müssten; somit stünden diese Maßnahmen im Wettbewerb zu Minderungsmaßnahmen anderer Sektoren, die weniger kostenintensiv sind. Die enorme Ausdehnung der Energiemaisflächen mit den damit verbundenen negativen Umweltwirkungen wie die hohe Stickstoffbelastung, das gesteigerte Erosionsrisiko und der Einfluss auf die Biodiversität muss mit den hohen Vermeidungsleistungen durch die gewonnene Bioenergie abgewogen

Tab.4.19 Akzeptanzmatrix. (Quelle: Thünen-Institut)

\begin{tabular}{|c|c|c|c|}
\hline & \multicolumn{3}{|l|}{ Strategien } \\
\hline & Klimaschutz & Bioenergie & Naturschutz \\
\hline Produzenten Agrarsektor & - & ++ & -- \\
\hline Politik & + & - & + \\
\hline Umweltschutz & ++ & - & ++ \\
\hline
\end{tabular}

Erwartete Akzeptanz: +: Akzeptanz, ++: hohe Akzeptanz, -: Ablehnung, --: hohe Ablehnung 
werden. Aufgrund der intensiven, negativen Umweltwirkungen dürfte diese Strategie in der öffentlichen Diskussion bei Vertretern des Umwelt- und Naturschutzes auf nur eingeschränkte Akzeptanz stoßen.

Die Natur- und Umweltschutzstrategie schreibt eine Mindestflächenstilllegung vor. Diese führt bundesweit zu einer Verringerung der Produktionsfläche, was sich positiv auf die Emissionsreduzierung und andere Umweltindikatoren auswirkt und die gesellschaftliche Akzeptanz der Strategie fördern dürfte. Für die Landwirtschaft sind solche restriktiven Maßnahmen wenig attraktiv, da sie ihre Anpassungsmöglichkeiten begrenzen. Unter Umständen erfolgt eine Intensivierung der Produktion auf den nicht stillgelegten Flächen, um die Produktionsverluste zu kompensieren. Dann wären auch die positiven Aspekte für den Umweltschutz in Frage gestellt. Für den Staat ergeben sich zwar Ausgaben für die Wiedervernässung, aber die zahlreichen, damit verbundenen positiven Umweltwirkungen dürften für Zustimmung sorgen.

Bei Überlegungen zu geeigneten Maßnahmen und Strategien muss immer beachtet werden, dass ihre hiesige Umsetzung anderenorts zu Landnutzungsänderungen (iLUC) und Intensivierungen führen kann, die weitreichende, negative Effekte mit sich ziehen können.

\section{Fazit Landwirtschaft}

Die untersuchten Maßnahmen folgen unterschiedlichen Vermeidungsansätzen: der quellenbezogenen THG-Minderung, der Substitution fossiler Energien und der $\mathrm{CO}_{2}$ Sequestierung. Dabei unterscheiden sich die Maßnahmen deutlich in ihren Vermeidungsleistungen und den Auswirkungen auf Umwelt, landwirtschaftliche Produktion und Einkommen sowie in ihrer regionalen Umsetzbarkeit. Die Indikatoren zur Flächen- und Kosteneffizienz ermöglichen einen ökonomischen Vergleich der Maßnahmen.

Mit Hinblick auf die Flächen- und Kosteneffizienz schneiden die Wiedervernässung von organischen Böden (mit und ohne Paludikulturanbau) und die Aufforstung gut ab. Diese Maßnahmen haben einen relativ geringen Einfluss auf die Produktion sowie das landwirtschaftliche Einkommen und bringen positive Umweltwirkungen. Allerdings sind diese Maßnahmen regional nur begrenzt umsetzbar. Die Maßnahmen, welche deutschlandweit umgesetzt werden können (Energiemaisanbau, Stickstoffabgabe und Flächenstilllegung), zeigen relativ hohe Vermeidungskosten und haben zum Teil deutlich negative Auswirkungen auf die landwirtschaftliche Produktion und Einkommen (Flächenstilllegung, Stickstoffabgabe) oder die Umwelt (Energiemaisproduktion).

Die Umsetzung von Vermeidungsmaßnahmen sollte daher in Kombination erfolgen, in denen sich die Vorteile der kombinierten Maßnahmen ergänzen bzw. sich die Nachteile zum Teil aufheben. Desweiteren sind neben der sektoralen Betrachtung regionale Wirkungsanalysen anzustellen.

In den untersuchten Strategien werden Einzelmaßnahmen kombiniert, die aufgrund ihrer Vermeidungsansätze und Wirkungen in die jeweilige Strategie passen. Die Klimaschutzstrategie kombiniert die kosteneffizientesten regionalen Maßnahmen (Wiedervernässung organischer Böden und Aufforstung) mit der deutschlandweit 
umsetzbaren Stickstoffabgabe. Die Kombination dieser ausgewählten Maßnahmen führt zur höchsten Flächen- und Kosteneffizienz der untersuchten Strategien, und ist somit durch einen effizienten Klimaschutz gekennzeichnet. In der Strategie „Bioenergie“ wird die Produktion von Biomasse zur Energiegewinnung gefördert. Die Vermeidungswirkung wird vornehmlich durch die Substitution fossiler Energieträger erzielt. Bei mittlerer Flächeneffizienz ist diese Strategie vorzüglich für die Erhaltung des landwirtschaftlichen Einkommens, hat allerdings durch den intensiven Pflanzenbau negative Auswirkungen auf die Umwelt. Durch den hohen Förderbedarf der Biomasseproduktion ist die Strategie durch mittlere Vermeidungskosten gekennzeichnet. Die Strategie „Natur- und Umweltschutz" erreicht die Vermeidung durch eine verpflichtende Flächenstilllegung und durch Förderung von Moorwiedervernässung. Beide Maßnahmen haben positive Auswirkungen auf die Umwelt, allerdings wirken sich die produktionsmindernden Effekte stark negativ auf das landwirtschaftliche Einkommen aus. Die Strategie mit dem größten Flächenanspruch erreicht die geringste Flächeneffizienz und die geringste Kosteneffizienz.

Neben der Sektoranalyse sollte zur vollständigen Bewertung der einzelnen Maßnahmen und Strategien eine regionale Wirkungsanalyse vertieft werden. Des Weiteren ist zu beachten, dass die Präferenz von Maßnahmen und Strategien stark von den jeweils betroffenen Interessensgruppen abhängt. Die Natur- und Umweltschutzstrategie repräsentiert deutlich Ziele des Umweltschutzes und führt zu geringeren landwirtschaftlichen Einkommen. Die Strategie „Bioenergie“ begünstigt die Einkommenserhaltung der Landwirte, aber behindert den Umweltschutz. Als Strategie, welche die Produzenten wenig benachteiligt und den Umweltschutz wenig begünstigt, kann die Klimaschutzstrategie bewertet werden, welche gleichzeitig auch die flächen- und kosteneffizienteste Strategie darstellt.

\subsection{Analyse der forstlichen Landnutzung}

Nils Ermisch, Hermann Englert, Margret Köthke und Peter Elsasser

\section{Zusammenfassung}

In Abschn. 4.6 werden Datenbasis, zusätzliche Annahmen und Simulationsergebnisse der forstlichen Referenzprojektion auf Basis des Modells FoBeSiMo für den Modellierungszeitraum 2015-2055 beschrieben und erläutert, und anschließend vier alternative Bewirtschaftungsstrategien (,Klimaschutz“, „Bioenergie“, „Umwelt- und Naturschutz“ sowie „Klimaanpassung“) gegenübergestellt. In zwei Exkursen wird zusätzlich auf die Auswirkungen von Aufforstungen sowie von Nutzungsverzichten eingegangen. Holzeinschläge, Deckungsbeiträge sowie Struktur und Höhe der Kohlenstoffsequestrierung verändern sich im Zeitverlauf und unterscheiden sich teilweise deutlich zwischen den vier Strategien. 


\subsubsection{Forstliche Referenzprojektion}

Hauptziel der forstbetrieblichen Untersuchung ist es, die Rohholzproduktion und darauf aufbauend finanzielle Erträge sowie Kohlenstoffspeicherung in Wald und Holz für jede Landnutzungsstrategie $\mathrm{zu}$ berechnen und $\mathrm{zu}$ bewerten. Die Referenzprojektion bildet hierbei den Zustand und die Entwicklung des Waldes unter der aktuellen Waldbewirtschaftung ab und schreibt diese unter gleichbleibenden Verhältnissen fort. Dabei geben die jeweiligen Waldbaulichen Richtlinien (WBRL) in den Bundesländern die Rahmenbedingungen der Waldbewirtschaftung für den Staatsforst vor. Für kommunale und private Waldbesitzer sind die WBRL nicht verbindlich; gleichwohl bieten sie Orientierungshilfen auch für diese Waldbesitzer. Für die forstbetrieblichen Simulationen wurden die in Tab. 4.20 beschriebenen ökonomischen Rahmenbedingungen unterstellt.

Ausgangspunkt der Analysen ist die in Abschn. 4.4.1 ermittelte Waldfläche von rund 11 Mio. ha in der Referenzprojektion. Für jeden Modellbetrieb (d.h. jedes der 13 Flächenbundesländer) liegt eine eigene, auf den in Abschn. 3.3 erläuterten Bewirtschaftungsmaßnahmen basierende Bewirtschaftung in der Referenzprojektion vor. Im Folgenden werden jeweils die auf das gesamte Bundesgebiet aggregierten Ergebnisse dargestellt. Zur Einordnung der nachfolgend beschriebenen Ergebnisse wird vorab auf einige modellimmanente Besonderheiten hingewiesen:

1. Der Modellierungshorizont von (nur) 40 Jahren $^{13}$ hat Auswirkungen auf die ökonomische Bewertung und damit auf die Interpretation der forstlichen Ergebnisse. Insbesondere die Auswirkungen langfristiger Investitionen (z. B. Baumartenwechsel) fallen dadurch nur z. T. in den Betrachtungszeitraum. Beispielsweise werden zwar die anfänglichen Begründungskosten, nicht jedoch die späteren Enderträge solcher Investitionen vollumfänglich erfasst.

2. Das Zusammenspiel des Waldwachstums- mit dem Betriebssimulator erforderte eine pragmatische Herangehensweise bezüglich der jeweiligen Eingangsannahmen. Es wird in den Modellierungen des Waldwachstums davon ausgegangen, dass die Landnutzungsstrategien bereits zu Beginn der Modellierung umgesetzt sind. Daraus folgend wurde für jede Hauptbaumart (Startalter 30 Jahre) auf jedem Standort in jedem Klimaszenario eine Wachstumskurve berechnet (vgl. Abschn. 4.2.2.4) Dadurch liegt der Fokus auf den Auswirkungen der Strategien. Die Endnutzung - als primäre wirtschaftliche Entscheidung - wird vom Betriebssimulator über die Zielstärke gesteuert. Da die flächenmäßige Altersklassenausstattung im Startjahr 2015 der BWI entspricht, startet die Modellierung mit Beständen, deren Bäume teilweise die in den Strategien

\footnotetext{
${ }^{13}$ Dies geht auf den allgemeinen Betrachtungshorizont des vorliegenden Projektes zurück, welcher mit 40 Jahren für forstliche Verhältnisse relativ kurz ist. Eine längere Simulationszeit wäre zwar modelltechnisch möglich gewesen, wäre aber nicht mit dem genannten Betrachtungshorizont kompatibel und hätte den verfügbaren Zeitrahmen gesprengt.
} 
Tab.4.20 Ökonomische Annahmen der forstbetrieblichen Berechnungen. (Quelle: N. Ermisch)

\begin{tabular}{l|r|r|r|r}
\hline & Eiche & Buche & $\begin{array}{l}\text { Fichte } \\
\text { (Douglasie) }\end{array}$ & Kiefer \\
\hline Holzerntekostenfreier Erlös [€/Fm] & 57,1 & 27,8 & 41,7 & 29,5 \\
\hline Anteil nvD-Holz [\%] & 7,6 & 7,6 & 3,6 & 3,6 \\
\hline Kulturkosten bei Baumartenerhalt [€/ha] & 3800 & 1460 & 620 & 2850 \\
\hline Kulturkosten bei Baumartenwechsel [€/ha] & 7600 & 7300 & 3100 & 5700 \\
\hline Läuterungskosten [€/ha] & 500 & 500 & 500 & 500 \\
\hline Umrechnung Volumen- in Erntefestmeter & 0,82 & 0,74 & 0,80 & 0,75 \\
\hline
\end{tabular}

$\mathrm{Fm}=$ Festmeter, nvD-Holz $=$ nicht verwertbares Derbholz

vorgegebenen Zielstärken überschreiten. Dadurch ergeben sich in diesen Strategien hohe Anfangseinschläge in der ersten Periode und entsprechend hohe Umbau- und Wiederbegründungskosten in der darauffolgenden Periode. Je niedriger dabei die Zielstärken der jeweiligen Bewirtschaftungsstrategie im Vergleich zur Referenzprojektion sind, desto höher sind die in der ersten Periode eingeschlagenen Holzmengen sowie die Wiederbegründungskosten. Dies ist durchaus realistisch, da beim Wechsel von einer Bewirtschaftungsstrategie zu einer anderen (beispielsweise zu einer Strategie mit geringeren Umtriebszeiten/Zielstärken) tatsächlich entsprechende Überhänge anfallen würden.

3. In der Referenzprojektion wurde als Durchforstungskonzept die Z-Baumdurchforstung gewählt, da diese von Forstbetrieben überwiegend durchgeführt wird. In den vier Bewirtschaftungsstrategien wurde hingegen die systematische Hochdurchforstung angewandt. Das Problem der hohen Anfangseinschläge in der ersten 5-Jahres-Periode konnte durch dieses Vorgehen abgeschwächt werden. Der Vergleich der Referenzprojektion mit den vier Landnutzungsstrategien wird dadurch jedoch erschwert, Vergleiche zwischen den Strategien sind hiervon nicht betroffen.

Die Ergebnisse für die Referenzprojektion zeigen (Abb. 4.38, 4.39 und 4.40), dass die Naturalproduktion und infolgedessen auch die Holzeinschläge, die finanziellen Erträge und die Kohlenstoffvorräte in Wald und Holz unter Klimaerwärmung (Szenario „RCP 8.5“) geringer ausfallen als bei gleichbleibendem Klimatrend ohne Temperaturerhöhung (Szenario 0K). Für den Betrachtungszeitraum bedeutet dies, dass unter jetziger Baumartenverteilung wie auch unter einer der alternativ untersuchten Baumartenverteilungen eine Klimaerwärmung um konstante $2{ }^{\circ} \mathrm{C} z u$ einem reduzierten Gesamtbaumwachstum führt.

Nach den Modellergebnissen werden in der Referenzprojektion zwischen etwa 65 und 80 Mio. $\mathrm{m}^{3}$ Holz pro Jahr eingeschlagen und damit etwa so viel wie in den letzten Jahren (BMEL 2014). Der erhöhte Holzeinschlag in der ersten Einschlagsperiode (vgl. Abb. 4.38) beruht darauf, dass Bäume im aktuellen Bestand laut BWI 2002 bereits 


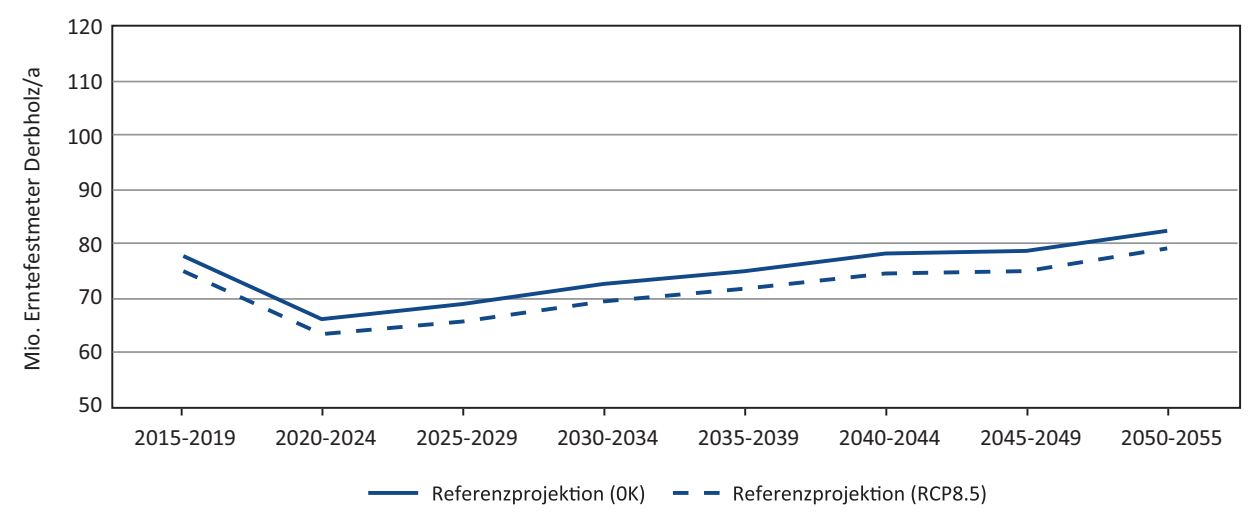

Abb. 4.38 Entwicklung des Rohholzeinschlages in Deutschland (Referenzprojektion für die Klimaszenarien 0K und RCP 8.5)

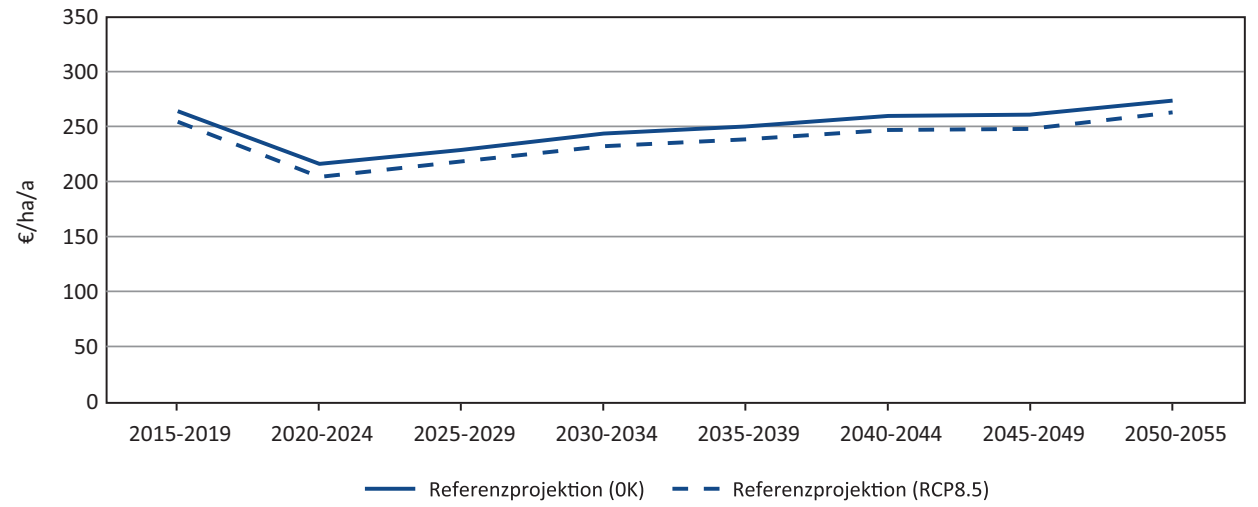

Abb.4.39 Entwicklung des Deckungsbeitrages I in Deutschland (Referenzprojektion für die Klimaszenarien 0K und RCP 8.5)

die in den Waldbaurichtlinien der Bundesländer angestrebten Zieldurchmesser überschritten haben. Die Ursachen dafür liegen u. a. in Naturschutzrestriktionen, EinzelbaumSchutzmaßnahmen und Einschränkungen der Befahrbarkeit von Beständen. Diese Spezialbedingungen konnten in den Modellanalysen nur bedingt berücksichtigt werden. Der Anstieg des Einschlages im Betrachtungszeitraum ergibt sich z. T. aus der aktuellen Altersklassenverteilung. In der Nachkriegszeit wurden Aufforstungen überwiegend mit Nadelholz durchgeführt. Dies bedingt, dass in Deutschland in den nächsten Jahren bzw. Jahrzehnten ein weiterer (zeitlich begrenzter) Vorratsaufbau im Wald zu erwarten ist, sofern die bisherigen Bewirtschaftungsgrundsätze weitergeführt werden. 


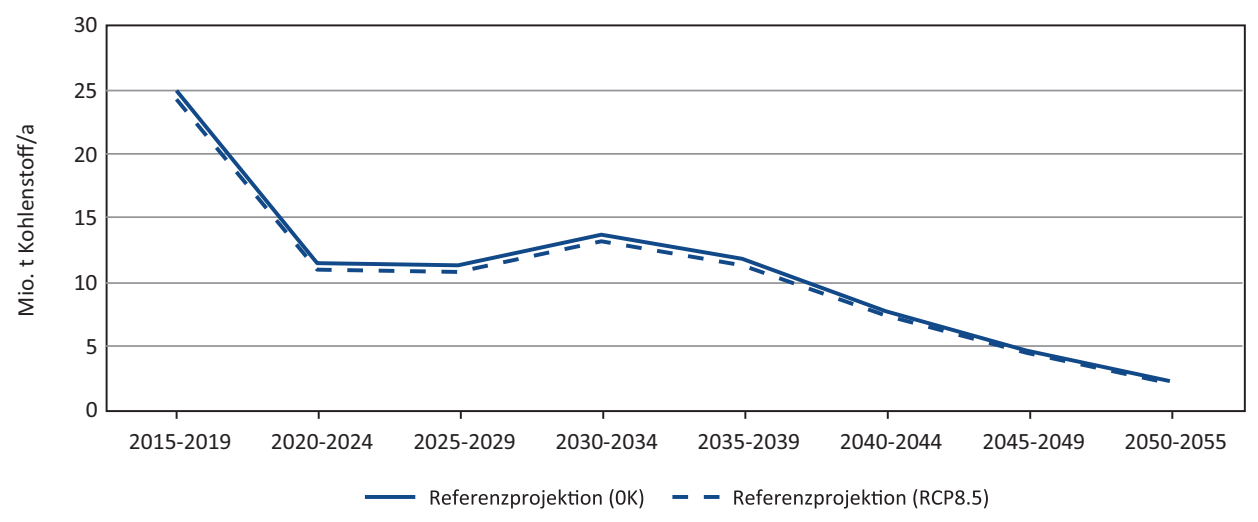

Abb.4.40 Entwicklung der forstlichen Senkenleistung (lebende Baumbiomasse, Totholz- und Holzproduktespeicher) ohne Substitution in Deutschland (Referenzprojektion für die Klimaszenarien „, $0 \mathrm{~K}^{\prime \prime}$ und „RCP 8.5“)

Die finanziellen Erträge der Modellbetriebe (vgl. Abb. 4.39) weisen im Betrachtungszeitraum einen ähnlichen Verlauf wie der Holzeinschlag auf. Durch die erhöhten Holzeinschläge realisieren die Modellbetriebe in der ersten 5-Jahres-Periode (2015-2019) höhere Erlöse und dadurch auch höhere Deckungsbeiträge. Dagegen werden in der zweiten 5-Jahres-Periode (2020-2025) die niedrigsten Deckungsbeiträge pro Hektar generiert, was auf die Wiederbegründungskosten ${ }^{14}$ der zuvor endgenutzten Bäume zurückzuführen ist. Die Deckungsbeiträge (DB I) der Modellbetriebe liegen zwischen 200 bis $250 € / \mathrm{ha}^{*} \mathrm{a}^{-1}$ und erreichen das Niveau der im Testbetriebsnetzes Forst des BMEL ausgewiesenen Ergebnisse (Ermisch et al. 2014). Wie schon beim Holzeinschlag, zeigt sich beim Deckungsbeitrag I im Vergleich des 0K-Szenarios und des RCP 8.5-Szenarios, dass die Deckungsbeiträge unter Klimaerwärmung geringer ausfallen.

Die Kohlenstoffsenkenleistung in der lebenden Baumbiomasse, im Totholz- und im Holzproduktespeicher nimmt bedingt durch die Altersstruktur der Wälder über den gesamten Betrachtungszeitraum ab (vgl. Abb.4.40) und geht gegen Ende des Betrachtungszeitraumes gegen null. Für die spätere Zukunft ist sogar eine negative Senkenleistung zu erwarten, d. h., der Wald wird dann per Saldo Kohlenstoff-Emissionen verursachen (vgl. Abschn. 2.3.2.2). Bei einer Klimaerwärmung, wie im Szenario „RCP 8.5“ wäre dieser Speicheraufbau durchgehend geringfügig schwächer ausgeprägt.

Bei der Interpretation der Entwicklung der Kohlenstoffspeicherung in Wald und Holz sei darauf hingewiesen, dass die in lebender Baumbiomasse, Holzproduktespeicher und Totholz akkumulierten Kohlenstoffmengen der Vorperioden nicht berücksichtigt wurden. Dies ermöglicht einen besseren Überblick über die verschiedenen Entwicklungen der

\footnotetext{
${ }^{14}$ Hier sind ausschließlich Wiederbegründungskosten mit der identischen Baumart berücksichtigt; Waldumbau findet in der Referenzprojektion nicht statt.
} 
Senkenleistung in den Strategien im Betrachtungszeitraum. Jedoch besteht auch die Problematik, dass den in der ersten Periode anfallenden Totholz- und Holzproduktemengen nicht der Abbau der Totholz- und Holzproduktespeicher der Vorperioden entgegengerechnet wird; das führt in den ersten Jahren zu einem (erhöhten) Aufbau dieser beiden Speicher. Im Laufe des Betrachtungszeitraums nivelliert sich dies, da sowohl Totholz wie auch Holzprodukte - aufgrund verwendeter Abbauraten - diese Speicherkompartimente auch wieder verlassen. Dies kann ein Grund sein, warum im Vergleich zu früheren Projektionen der zukünftigen Senkenleistung (z. B. Dieter et al. 2005) das Niveau in dieser Modellierung insgesamt höher ist und bis 2055 noch nicht unter null absinkt. Beides kann auch darauf zurückzuführen sein, dass die hier berechnete Senkenleistung auch den Speicheraufbau in Holzprodukten und Totholz berücksichtigt, wogegen frühere Berechnungen häufig allein auf die Entwicklung der Baumbiomassespeicher beschränkt waren.

\subsubsection{Strategien im Bereich „Forstwirtschaft“}

Aufgrund der langen Produktionszyklen in der Forstwirtschaft und langfristigen Waldumbauprozesse im Zeitverlauf beeinflussen sich die einzelnen in Abschn. 3.3 erläuterten waldbaulichen Maßnahmen gegenseitig. Wirkungsanalysen isolierter Einzelmaßnahmen sind daher - abgesehen von einigen Ausnahmen wie dem Nutzungsverzicht (Stilllegung) oder Erstaufforstungen (Neubegründungen) - wenig sinnvoll und wurden für die Forstwirtschaft nicht durchgeführt. Ferner dürften isolierte waldbauliche Maßnahmen nur geringe und in der Regel für die waldbauliche Praxis nicht zu interpretierende Effekte bewirken. Vor diesem Hintergrund wurden die untersuchten forstlichen Bewirtschaftungsmaßnahmen jeweils zu in sich konsistenten Maßnahmenbündeln zusammengefasst, die die nachfolgend erläuterten Strategien repräsentieren.

\subsubsection{Ausgestaltung der Strategien}

In Rahmen der Strategien „Klimaschutz“, „Bioenergie“, „Natur- und Umweltschutz“ sowie „Klimaanpassung“ werden unterschiedliche Ziele der Waldnutzung untersucht. Um diese Ziele zu erreichen, wurden die in Abschn. 3.3 beschriebenen waldbaulichen Maßnahmen jeweils unterschiedlich ausgestaltet und aufeinander abgestimmt. Einen Überblick über die resultierenden waldbaulichen Bewirtschaftungsregime in den betrachteten Strategien vermittelt Tab. 4.21. Diese Bewirtschaftungsregime stellen bundesweit einheitliche Änderungen der Waldbewirtschaftung gegenüber der Referenzprojektion dar. Die Waldbewirtschaftung erfolgt in der Referenzprojektion betriebsspezifisch und regional unterschiedlich. Während für die Nutzung des Privatwaldes betriebsspezifische Prioritäten im Vordergrund stehen, gelten für die Bewirtschaftung des Staatswaldes in den Bundesländern jeweils Richtlinien (WBRL), die in WEHAM berücksichtigt sind. 
Tab.4.21 Zuordnung der untersuchten forstlichen Einzelmaßnahmen zu Strategien. (Quelle: N. Ermisch)

\begin{tabular}{|c|c|c|c|c|c|}
\hline Maßnahmen & $\begin{array}{l}\text { Referenz- } \\
\text { projektion }\end{array}$ & Klimaschutz & Bioenergie & $\begin{array}{l}\text { Natur- und } \\
\text { Umwelt- } \\
\text { schutz }\end{array}$ & $\begin{array}{l}\text { Klima- } \\
\text { anpassung }\end{array}$ \\
\hline $\begin{array}{l}\text { Baumarten- } \\
\text { wahl }\end{array}$ & Status quo & $\begin{array}{l}\mathrm{Dgl} \text { (bis } 15 \% \text { ) } \\
\text { für Ei und } \mathrm{Bu}\end{array}$ & $\begin{array}{l}\mathrm{Dgl}(\text { bis } 30 \% \text { ) } \\
\text { für Ei und Ki }\end{array}$ & $\begin{array}{l}\text { Bu (bis } 50 \% \text { ) } \\
\text { für Fi und Ki }\end{array}$ & $\begin{array}{l}\text { Bu (bis } 25 \% \text { ) } \\
\text { für Fi und Ei }\end{array}$ \\
\hline $\begin{array}{l}\text { Durch- } \\
\text { forstungs- } \\
\text { häufigkeit }\end{array}$ & $\begin{array}{l}\text { WBRL/ } \\
\text { WEHAM }\end{array}$ & 10 Jahre & 5 Jahre & 10 Jahre & 5 Jahre \\
\hline $\begin{array}{l}\text { Durch- } \\
\text { forstungs- } \\
\text { stärke }\end{array}$ & $\begin{array}{l}\text { Grundfl.- } \\
\text { leitkurve } \\
\text { (mäßig) }\end{array}$ & $+2,5 \%$ & $-10 \%$ & $+5 \%$ & $-7,5 \%$ \\
\hline Zielstärke & $\begin{array}{l}\text { WBRL/ } \\
\text { WEHAM }\end{array}$ & $\pm 0 \mathrm{~cm}$ & $-10 \mathrm{~cm}$ & $+5 \mathrm{~cm}$ & $-10 \mathrm{~cm}$ \\
\hline $\begin{array}{l}\text { Endnutzungs- } \\
\text { menge }\end{array}$ & $\begin{array}{l}\text { WBRL/ } \\
\text { WEHAM }\end{array}$ & $-2,5 \%$ & $+10 \%$ & $-5 \%$ & $+7,5 \%$ \\
\hline $\begin{array}{l}\text { Endnutzungs- } \\
\text { zeitraum }\end{array}$ & $\begin{array}{l}\text { WBRL/ } \\
\text { WEHAM }\end{array}$ & +5 Jahre & -20 Jahre & +10 Jahre & -15 Jahre \\
\hline $\begin{array}{l}\text { Holzbereit- } \\
\text { stellung }\end{array}$ & Status quo & $\begin{array}{l}\text { Bauholz } \\
\text { (mittlere ZS) }\end{array}$ & $\begin{array}{l}\text { Energieholz } \\
\text { (niedrige ZS) }\end{array}$ & $\begin{array}{l}\text { Starkholz } \\
\text { (hohe ZS) }\end{array}$ & $\begin{array}{l}\text { Bauholz } \\
\text { (mittlere ZS) }\end{array}$ \\
\hline $\begin{array}{l}\text { Nutzungsver- } \\
\text { zicht* }\end{array}$ & Status quo & Status quo & Status quo & $\begin{array}{l}10 \% \text { der } \\
\text { Waldfläche* }\end{array}$ & Status quo* \\
\hline $\begin{array}{l}\text { Erstauf- } \\
\text { forstung* }\end{array}$ & Status quo & $\begin{array}{l}10 \% \text { mehr } \\
\text { Wald* }\end{array}$ & Status quo & Status quo & Status quo \\
\hline $\begin{array}{l}\text { Ziel der } \\
\text { Strategie }\end{array}$ & & $\begin{array}{l}\text { Niedriger Va } \\
\text { Hoher Vv } \\
\text { Hohe Uz }\end{array}$ & $\begin{array}{l}\text { Sehr hoher Va } \\
\text { Sehr niedriger } \\
\text { Vv } \\
\text { Sehr niedrige } \\
\text { Uz }\end{array}$ & $\begin{array}{l}\text { Sehr niedriger } \\
\text { Va } \\
\text { Sehr hoher Vv } \\
\text { Sehr hohe Uz }\end{array}$ & $\begin{array}{l}\text { Hoher Va } \\
\text { Niedriger Vv } \\
\text { Niedrige Uz }\end{array}$ \\
\hline
\end{tabular}

*Die beiden Maßnahmen Erstaufforstung und Nutzungsverzicht wurden jeweils als abtrennbare Module der Klimaschutz- bzw. Natur- und Umweltschutzstrategie berechnet und sind in Abschn. 4.6.2.3 dargestellt

Die Referenzprojektion bezieht sich hier auf die Baumarten und Waldflächenverteilung der BWI 2002

$\mathrm{Dgl}=$ Douglasie, $\mathrm{Ei}=$ Eiche, $\mathrm{Bu}=$ Buche, $\mathrm{Fi}=$ Fichte, $\mathrm{Ki}=$ Kiefer, $\mathrm{WBRL}=$ Waldbaurichtlinie WEHAM $=$ Waldentwicklungs- und Holzaufkommensmodellierung, $\mathrm{sZS}=$ Zielstärke, $\mathrm{Va}=$ Vorrat ausscheidend, $\mathrm{Vv}=$ Vorrat verbleibend, $\mathrm{Uz}=\mathrm{Umtriebszeit}$

In der Klimaschutzstrategie besteht das Ziel, den Holzvorrat im Wald zu erhöhen. Dazu wird die Endnutzungsmenge gegenüber der Bewirtschaftung in der Referenzprojektion um 2,5\% verringert und der Endnutzungszeitraum um fünf Jahre verlängert. Eichen und Buchen werden bei einer vergleichsweise geringen Durchforstungshäufig- 
keit von zehn Jahren zu $15 \%$ durch Douglasien ersetzt. Zusätzlich wurde in der Klimaschutzstrategie untersucht, wie die Maßnahme „Erstaufforstung“ wirkt. Konkret wurde eine Ausdehnung der Waldfläche um $10 \%$ unterstellt (vgl. Tab. 4.21), auf deren Auswirkungen in Abschn. 4.6.2.2 eingegangen wird. Entsprechend der Maßnahmenvorgaben der Klimaschutzstrategie wurde diese Aufforstung zu $100 \%$ mit Douglasie durchgeführt.

Bei der Bioenergiestrategie hat eine hohe Biomasseproduktion Priorität, um möglichst viel Energieholz bereitzustellen. Das soll durch eine hohe Endnutzungsmenge, stark verkürzte Endnutzungszeiträume und niedrige Umtriebszeiten bei reduzierter Zielstärke erreicht werden. Es wird auf zuwachsstarke Baumarten wie die Douglasie gesetzt, die geerntete Eichen und Kiefern zu $30 \%$ ersetzen soll.

Das leitende Prinzip der Natur- und Umweltschutzstrategie ist der Aufbau hoher Vorräte im Bestand. Dazu werden hohe Umtriebszeiten bzw. Zielstärken avisiert, was mit einer vergleichsweise niedrigen Holznutzung einhergeht. Die Baumartenwahl orientiert sich an der in Deutschland aktuellen potenziellen natürlichen Vegetation und an hohen Laubholzanteilen, insbesondere der Buche. Zusätzlich wurde eine Flächenstilllegung von $10 \%$ der heutigen Waldfläche unterstellt.

In der Klimaanpassungsstrategie gilt es, klimabedingte Risiken zu vermeiden. Die Bestände sollen durch erhöhte Buchenanteile stabilisiert werden, die als Laubbäume weniger anfällig bei Herbststürmen sind und sich aufgrund geringerer Transpiration im Winterhalbjahr positiv auf die Grundwasserneubildung auswirken. Da mit zunehmendem Alter und Größe das klimabedingte Kalamitätsrisiko steigt, liegt der Fokus der Waldbewirtschaftung in der Klimaanpassungsstrategie auf kürzeren Nutzungszeitraumen, d. h. einer geringeren Zielstärke, höheren Endnutzungsmenge und verkürzten Endnutzungszeiträumen.

\subsubsection{Ergebnisse der forstbetrieblichen Modellierung für die Strategien}

Die Bewirtschaftungsmaßnahmen ergaben deutlich unterschiedliche Baumartenanteile in den Strategien (vgl. Tab. 4.22). Während die Baumartenanteile in der Klimaschutzstrategie im Vergleich zu 2015 nur geringfügige Unterschiede aufweisen, führten die Bewirtschaftungsmaßnahmen in den drei anderen Strategien teilweise zu erheblichen

Tab.4.22 Verteilung der Holzartengruppen in \% im Ausgangsjahr 2015 sowie in den untersuchten Strategien 2055. (Quelle: N. Ermisch)

\begin{tabular}{l|r|r|r|r|r}
\hline Maßnahmen & $\mathbf{2 0 1 5}$ & Klimaschutz & Bioenergie & Natur- und Umwelt & Klimaanpassung \\
\hline Eiche & 10 & 9 & 6 & 10 & 6 \\
\hline Buche & 31 & 29 & 31 & 46 & 52 \\
\hline Fichte & 30 & 30 & 29 & 21 & 13 \\
\hline Douglasie & 2 & 5 & 21 & 2 & 2 \\
\hline Kiefer & 27 & 27 & 13 & 21 & 27 \\
\hline Gesamt & $\mathbf{1 0 0}$ & $\mathbf{1 0 0}$ & $\mathbf{1 0 0}$ & $\mathbf{1 0 0}$ & $\mathbf{1 0 0}$ \\
\hline
\end{tabular}


Tab.4.23 Durchschnittlicher jährlicher Holzeinschlag, Deckungsbeitrag I und Kohlenstoffsequestrierung durch die ober- und unterirdische lebende Baumbiomasse in den Strategien (ohne Totholz- und Holzproduktespeicher und ohne Substitution; Zeitraum 2015-2055; jeweils für Klimaszenario 0K und RCP 8.5). (Quelle: N. Ermisch)

\begin{tabular}{|c|c|c|c|c|}
\hline Maßnahmen & Klima schutz ${ }^{\mathbf{a}}$ & Bioenergie & Natur- und Umwelt ${ }^{b}$ & Klima anpassung \\
\hline \multicolumn{5}{|l|}{$\mathbf{0 K}$} \\
\hline $\begin{array}{l}\text { Einschlag } \\
{[\text { EfmD/ha/a] }}\end{array}$ & 8,51 & 8,34 & 7,95 & 8,34 \\
\hline DB I [€/ha/a] & 289,33 & 271,96 & 252,33 & 236,58 \\
\hline $\begin{array}{l}\text { C-Sequestrierung } \\
\text { [tC/ha/a] }\end{array}$ & 0,374 & 0,290 & 0,552 & 0,183 \\
\hline \multicolumn{5}{|l|}{ RCP 8.5} \\
\hline $\begin{array}{l}\text { Einschlag } \\
{[\text { EfmD/ha/a] }}\end{array}$ & 8,10 & 8,11 & 7,51 & 8,03 \\
\hline DB I [€/ha/a] & 274,51 & 263,80 & 236,28 & 226,70 \\
\hline $\begin{array}{l}\text { C-Sequestrierung } \\
\text { [tC/ha/a] }\end{array}$ & 0,363 & 0,289 & 0,534 & 0,179 \\
\hline
\end{tabular}

EfmD $=$ Erntefestmeter Derbholz, DB I = Deckungsbeitrag I, C = Kohlenstoff

aOhne Modul „Erstaufforstung“

bohne Modul „Nutzungsverzicht““

Verschiebungen einzelner Baumartenanteile. Bei einer Umsetzung der Bioenergiestrategie wäre im Jahr 2055 mehr als jeder fünfte Baum eine Douglasie, die vor allem Kiefern, aber auch Eichen substituiert. In der Natur- und Umweltschutzstrategie und auch der Klimaanpassungsstrategie stellt sich der beabsichtigt hohe Laubbaumanteil ein. Dieser ist bei der Klimaanpassungsstrategie aufgrund der kürzeren Umtriebszeit, die einen früheren Baumartenwechsel ermöglicht, am höchsten.

Die Auswirkungen der untersuchten Strategien auf den Holzeinschlag, die finanziellen Konsequenzen für Forstbetriebe sowie die Kohlenstoffsequestrierung sind in Tab. 4.23 dargestellt. Die Auswirkungen weisen bei den Bewirtschaftungsregimen im zeitlichen Verlauf unterschiedliche Dynamiken auf. Um eine Vergleichbarkeit der Strategien herzustellen, werden die über den gesamten Betrachtungszeitraum von 2015 bis 2055 gemittelten durchschnittlich zu erwartenden jährlichen Effekte, jeweils für die beiden unterstellten Klimaszenarien, dargestellt. Trotz der Durchschnittsbildung über 40 Jahre wirken sich manche Effekte, auf die in den nachfolgenden Erläuterungen der Ergebnisse eingegangen wird, auch über den Betrachtungszeitraum hinaus aus.

Die mittleren jährlichen Holzeinschläge weisen in den vier Strategien ein ähnliches Niveau auf. Die Klimaschutzstrategie erzielt im Szenario „,K“ mit jährlich 8,5 Efm/ha den höchsten durchschnittlichen Einschlag, gefolgt von der Bioenergie- bzw. Klimaanpassungsstrategie, in denen etwas geringere Holzeinschläge realisiert werden. Die maßnahmebedingt 
niedrigsten Holzeinschläge in Höhe von $8 \mathrm{Efm} / \mathrm{ha}$ und Jahr sind in der Natur- und Umweltschutzstrategie zu verzeichnen. Somit zeigen die Bewirtschaftungsstrategien in Bezug auf den Holzeinschlag Unterschiede von bis zu $10 \%$. In allen Strategien führt eine Temperaturerhöhung wie im Szenario „RCP 8.5“ zu einem verringerten Holzeinschlag. Dieser Rückgang beträgt je nach Strategie 3 bis $5 \%$.

Betrachtet man die Entwicklung der Holzeinschläge über den Betrachtungszeitraum, so zeigen sich weitere Unterschiede zwischen den Strategien. In Abb. 4.41 lassen sich zum einen die unterschiedlichen Endnutzungsrhythmen von fünf bzw. zehn Jahren erkennen. Auffällig sind aber v. a. die sehr hohen Holzeinschläge im ersten 5-JahresZeitraum (2015-2019) der Bioenergie- sowie der Klimaanpassungsstrategie. Der Hauptgrund dafür sind die gegenüber dem Basisjahr 2015 bzw. der Referenzprojektion deutlich reduzierten Zielstärken, sodass durch den Bewirtschaftungswechsel viele der unter derzeitigen Bewirtschaftungszielen noch nicht hiebreifen Bäume eingeschlagen werden. Beispielsweise werden in der Bioenergiestrategie im zweiten 5-Jahres-Zeitraum (2020-2024) über 2 Mio. ha wieder aufgeforstet. Dies bewirkt in der Folge hohe Wiederbegründungskosten sowie niedrigere Zuwächse, deren Auswirkungen auf Holznutzung, finanzielle Erträge wie auch Kohlenstoffspeicherung während des Betrachtungszeitraumes nicht voll kompensiert werden.

$\mathrm{Ab}$ dem zweiten 5-Jahres-Zeitraum ist zu erkennen, dass in der Klimaschutzsowie der Natur- und Umweltschutzstrategie mehr Holz eingeschlagen wird als in der

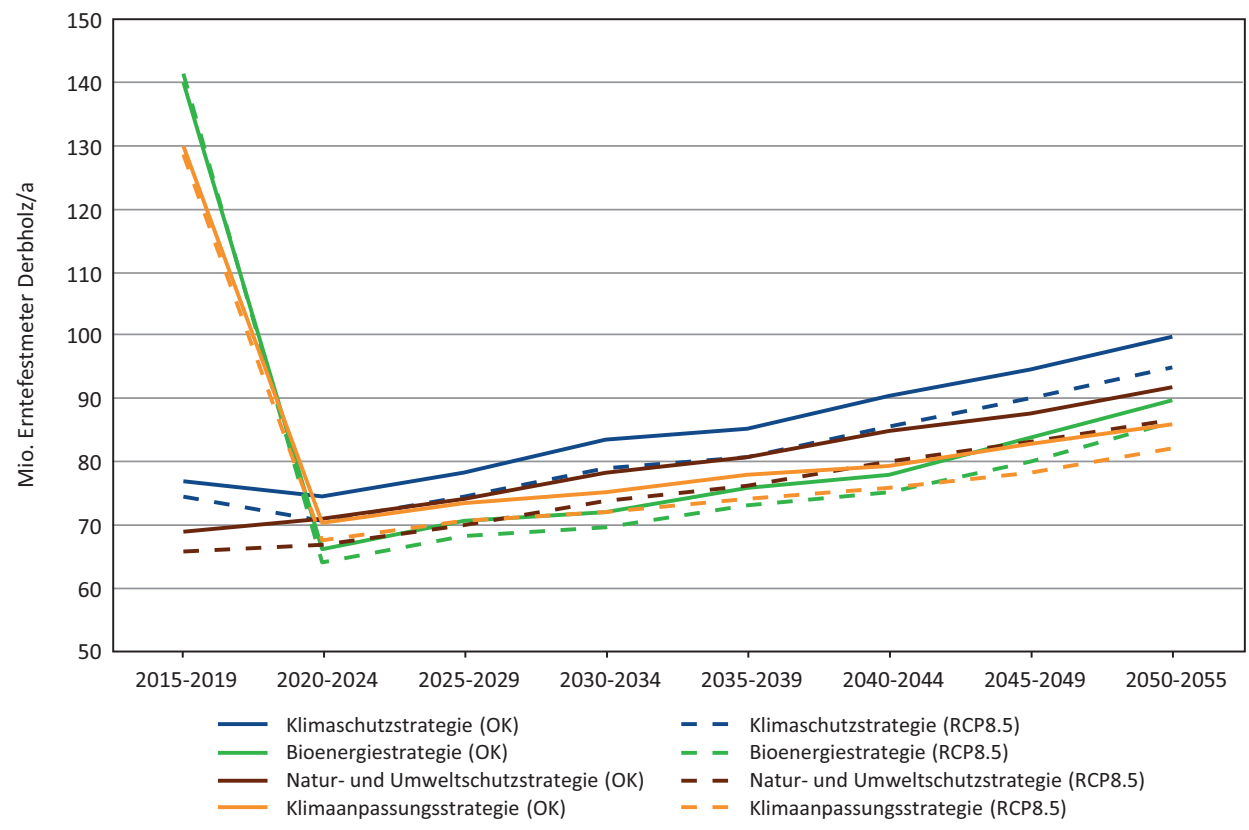

Abb. 4.41 Entwicklung des Holzeinschlages in Deutschland in den vier alternativen Bewirtschaftungsstrategien (2015-2055) 
Bioenergie- bzw. Klimaanpassungsstrategie. Das liegt vor allem an den hohen Einschlägen im ersten 5-Jahres-Zeitraum in der Bioenergie- bzw. Klimaanpassungsstrategie. Die dadurch entstehenden umfangreichen Wiederaufforstungsflächen produzieren in den Folgeperioden weniger erntereifes Holz. Auch der in der Bioenergiestrategie avisierte Wechsel zu ertragsstärkeren Baumarten kann dies innerhalb des Betrachtungszeitraums von 40 Jahren nicht kompensieren, da die Bäume das Erntealter innerhalb dieses Zeitraums trotz reduzierter Zielstärken nicht erreichen werden.

Grundsätzlich befinden sich die deutschen Wälder derzeit noch in einer Aufbauphase. Insofern verursachen die in der Klimaschutz- sowie Natur- und Umweltschutzstrategie festgelegten vergleichsweise gemäßigten Holznutzungen keine Diskontinuitäten innerhalb des Betrachtungszeitraums von 2015-2055. Des Weiteren sind in der Bioenergieund Klimaanpassungsstrategie die fünfjährigen Durchforstungsintervalle zu erkennen, die nach den Einstellungen des Waldwachstumssimulators SILVA auf eine Periode mit etwas niedrigeren Einschlägen immer eine Periode mit etwas höheren Einschlägen folgen lässt.

Die finanziellen Auswirkungen auf die forstwirtschaftlichen Betriebe stellen sich gemessen am mittleren jährlichen Deckungsbeitrag im Betrachtungszeitraum in der Klimaschutzstrategie am vorzüglichsten dar und liegen bei rund $289 €$ je ha und Jahr, gefolgt von der Bioenergiestrategie (vgl. Tab. 4.23). Die Deckungsbeiträge bei der Natur- und Umweltschutz- sowie Klimaanpassungsstrategie fallen gegenüber der Klimaschutzstrategie um 13 bzw. $18 \%$ geringer aus. Die niedrigen gesamtbetrieblichen Deckungsbeiträge ergeben sich aus den baumartspezifisch unterschiedlichen Kosten und Erlösen (vgl. Tab. 4.20) und den jeweiligen Anteilen der Baumarten. So sind die Naturund Umweltschutz- sowie die Klimaanpassungsstrategie durch einen hohen Anteil an Buchen gekennzeichnet, deren Deckungsbeitrag im Vergleich zu Fichten niedriger ist (vgl. Tab. 4.23).

Die durchschnittlichen Deckungsbeiträge entwickeln sich bei den Strategien im Betrachtungszeitraum sehr unterschiedlich. Während sie in der Klimaschutz- sowie Natur- und Umweltschutzstrategie nahezu kontinuierlich steigen, führen die hohen Holzeinschläge in der ersten 5-Jahres-Periode der Bioenergie- und Klimaanpassungsstrategie zu hohen Deckungsbeiträgen (vgl. Abb. 4.42). Diese gehen jedoch in der zweiten 5-Jahres-Periode vorübergehend auf 150 bzw. 62 €/ha zurück, vor allem aufgrund der umfangreichen Wiederaufforstungsflächen, die hohe Bestandesbegründungskosten verursachen. Diese sind in der Klimaanpassungsstrategie trotz der etwas kleineren Wiederaufforstungsfläche von 1,8 Mio. ha gegenüber ca. 2 Mio. ha in der Bioenergiestrategie höher, weil die Erhöhung des Buchenanteils in der Klimaanpassungsstrategie sowohl bei Baumartenwechsel als auch bei Baumartenerhalt höhere Kulturkosten bedingt (vgl. dazu Tab. 4.20).

Ab der zweiten 5-Jahres-Periode werden durchgehend bis zum Jahr 2055 in der Klimaschutzstrategie die höchsten Deckungsbeiträge erzielt. Das liegt vor allem an den vergleichsweise geringen Anfangseinschlägen und Umbaukosten, wodurch konstant hohe Einschläge erzielt werden können. Mit einem moderaten Umbau hin zur Douglasie 


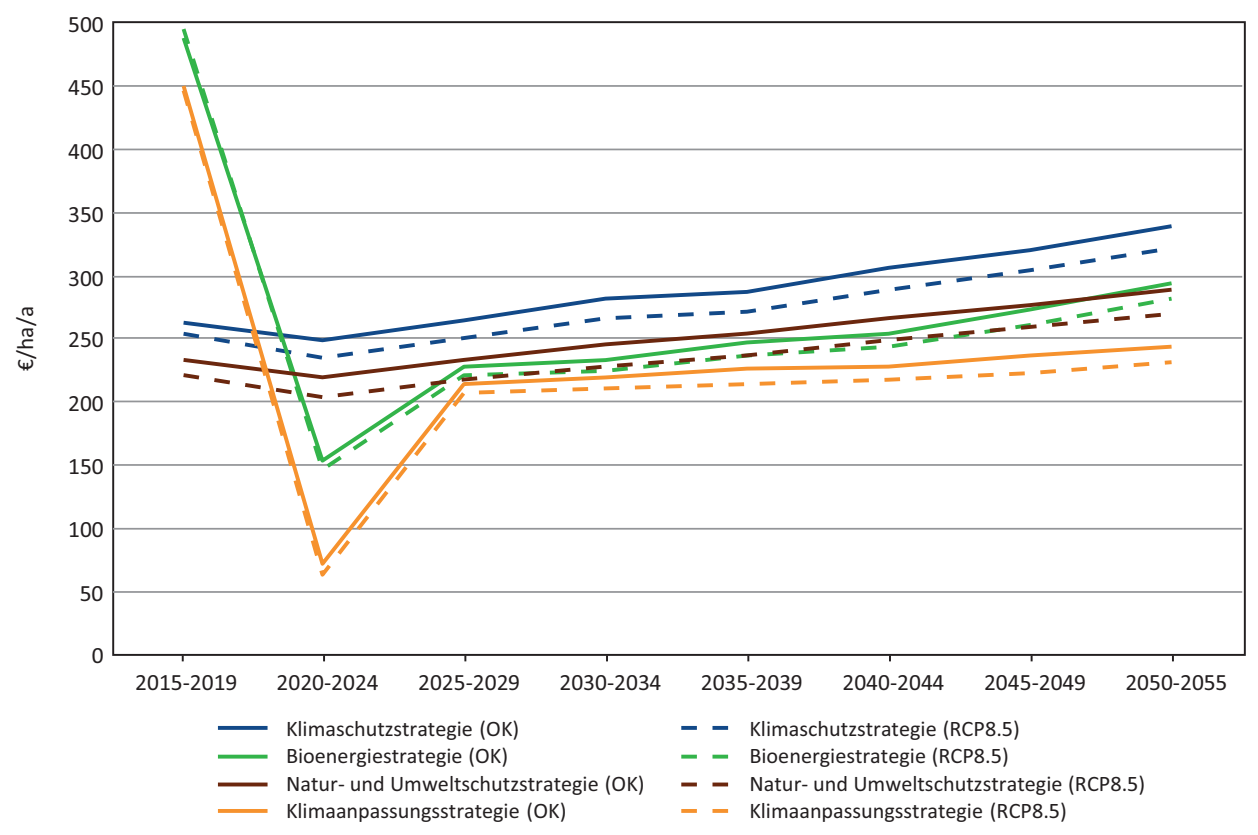

Abb. 4.42 Entwicklung des Deckungsbeitrages I in Deutschland in den vier alternativen Bewirtschaftungsstrategien (2015-2055)

und den nicht veränderten Zielstärken profitieren die Forstbetriebe in dieser Strategie deutlich von den sich derzeit im Wuchsoptimum befindlichen Fichtenbeständen der Nachkriegsaufforstungen, die innerhalb des Betrachtungszeitraums die Hiebsreife erreichen. Dagegen erreichen die Bestände in der Natur- und Umweltschutzstrategie aufgrund der um $5 \mathrm{~cm}$ erhöhten Zielstärken den angestrebten Zieldurchmesser nur teilweise im Betrachtungszeitraum. Die Berücksichtigung einer Klimaerwärmung führt zu keinen strukturellen Änderungen der Ergebnisse. Die Deckungsbeiträge liegen beim RCP 8.5-Szenario durchgehend etwas niedriger als beim 0K-Szenario.

Die Berechnung der durchschnittlichen jährlichen Kohlenstoffsenken- und -substitutionsleistung 2015-2055 (vgl. Tab. 4.24 sowie Abb. 4.43) ist mit dem von Schweinle et al. (2018) beschriebenen Modell durchgeführt worden. Es sei daran erinnert, dass der bis 2015 aufgebaute Kohlenstoffspeicher in keinem der Speicherkompartimente außer der lebenden Baumbiomasse berücksichtigt wurde, um so eine bessere Vergleichbarkeit der Strategien zu gewährleisten. Die Abb. 4.43 zeigt, dass die durchschnittliche jährliche Senken- und Substitutionsleistung in den Strategien „Klimaschutz“ sowie „Natur- und Umweltschutz“ am höchsten, in den Strategien „Klimaanpassung“ und „Bioenergie“ dagegen am geringsten ist. Auf die einzelnen Kompartimente entfallen dabei sehr unterschiedliche Anteile. 
Tab. 4.24 Durchschnittliche jährliche Kohlenstoffsenken- und -substitutionsleistung in den vier alternativen Bewirtschaftungsstrategien (2015-2055). (Quelle: N. Ermisch)

\begin{tabular}{|c|c|c|c|c|c|c|}
\hline & \multicolumn{3}{|c|}{ Speicherveränderungen } & \multicolumn{2}{|c|}{ Substitution } & \multirow[t]{2}{*}{ Summe } \\
\hline & Biomasse & $\begin{array}{l}\text { Totholz- } \\
\text { speicher }\end{array}$ & HWP & Material & Energie & \\
\hline $\begin{array}{l}\text { Bioenergie- } \\
\text { strategie }(0 \mathrm{~K})\end{array}$ & 2.923 .375 & 4.241 .878 & 5.133 .309 & 17.332 .690 & 9.871 .979 & 39.503.231 \\
\hline $\begin{array}{l}\text { Bioenergie- } \\
\text { strategie } \\
\text { (RCP8.5) }\end{array}$ & 2.917 .807 & 4.128 .135 & 4.983 .388 & 16.957 .688 & 9.665 .236 & 38.652.255 \\
\hline $\begin{array}{l}\text { Klima- } \\
\text { anpassungs- } \\
\text { strategie }(0 \mathrm{~K})\end{array}$ & 1.847 .919 & 4.378 .579 & 5.304 .408 & 17.629 .309 & 10.000 .322 & 39.160 .539 \\
\hline $\begin{array}{l}\text { Klima- } \\
\text { anpassungs- } \\
\text { strategie } \\
\text { (RCP8.5) }\end{array}$ & 1.807 .660 & 4.222 .584 & 5.107 .402 & 17.065 .230 & 9.670 .242 & 37.873.117 \\
\hline $\begin{array}{l}\text { Klimaschutz- } \\
\text { strategie }(0 \mathrm{~K})\end{array}$ & 3.776 .366 & 5.399 .158 & 5.744 .562 & 17.501 .088 & 9.600 .385 & 42.021.559 \\
\hline $\begin{array}{l}\text { Klimaschutz- } \\
\text { strategie } \\
\text { (RCP8.5) }\end{array}$ & 3.667 .073 & 5.214 .428 & 5.518 .224 & 16.837 .092 & 9.208 .108 & 40.444.924 \\
\hline $\begin{array}{l}\text { Natur- und } \\
\text { Umweltschutz- } \\
\text { strategie }(0 \mathrm{~K})\end{array}$ & 5.568 .644 & 5.385 .579 & 5.430 .186 & 16.485 .949 & 8.979 .449 & 41.849 .808 \\
\hline $\begin{array}{l}\text { Natur- und } \\
\text { Umweltschutz- } \\
\text { strategie } \\
\text { (RCP8.5) }\end{array}$ & 5.388 .582 & 5.183 .587 & 5.185 .817 & 15.745 .537 & 8.536 .656 & 40.040 .179 \\
\hline
\end{tabular}

Angaben in $\mathrm{t} \mathrm{C} / \mathrm{a}$

Insbesondere die Unterschiede der durchschnittlichen jährlichen Senkenleistung der lebenden Baumbiomasse sind deutlich zu erkennen. Diese belaufen sich in der Natur- und Umweltschutz- sowie der Klimaschutzstrategie auf jährlich knapp 0,6 bzw. knapp 0,4 tC je ha und Jahr. Die hohen Senkenleistungen sind zum einen durch die deutlich höhere Ausgangsvorräte in den beiden Strategien zu erklären und zum anderen dadurch, dass keine umfangreichen Aufforstungsflächen in der ersten 5-Jahres-Periode entstanden sind wie in der Bioenergie- und Klimaanpassungsstrategie.

Auch der Totholzspeicher ist aufgrund der anfänglich höheren Totholzanteile in der Natur- und Umweltschutz- sowie in der Klimaschutzstrategie größer als in der Bioenergie- und der Anpassungsstrategie (vgl. Abb. 4.43). Dies liegt daran, dass die Totholzmenge im Modell stark von der Höhe des Anfangsvorrates an Baumbiomasse abhängt. 


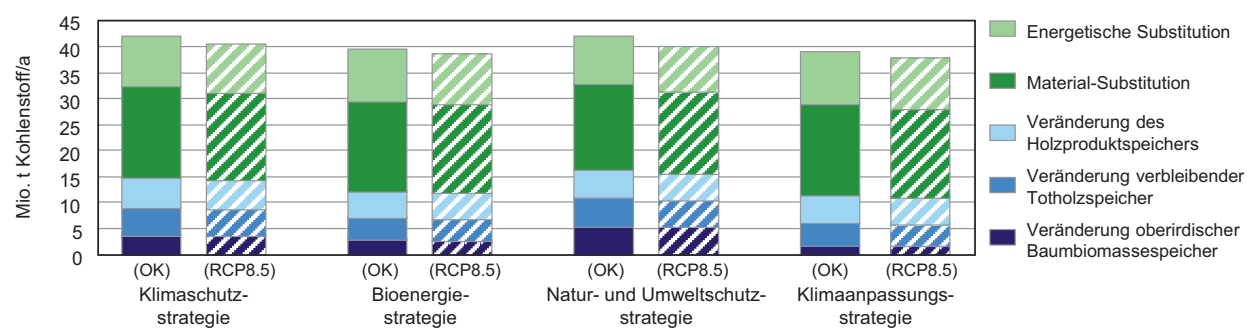

Abb. 4.43 Durchschnittliche jährliche Kohlenstoffsenken- und -substitutionsleistung in den vier alternativen Bewirtschaftungsstrategien (2015-2055)

Die durchschnittliche Senkenleistung des Holzproduktespeichers weist dagegen keine größeren Unterschiede zwischen den Strategien auf. Der größte Teil der Senkenleistung entfällt auf die Materialsubstitution und die energetische Substitution. Die jeweiligen Substitutionspotenziale unterscheiden sich zwischen den Strategien jedoch vergleichsweise wenig und können daher die Unterschiede der Speicherung in der Baumbiomasse nicht vollständig kompensieren, zumindest nicht während des Betrachtungszeitraums. Die durchschnittliche jährliche Kohlenstoffsenken- und -substitutionsleistung des RCP 8.5-Szenarios liegt jeweils unterhalb der des 0K-Szenarios. Inwieweit sich dies nach dem Betrachtungszeitraum ändern wird, kann aus den Ergebnissen des Modells nicht abgeleitet werden.

\subsubsection{Exkurs: Klimaschutz durch Ausdehnung der Waldfläche und durch Nutzungsverzichte im Wald}

\section{Modul „Aufforstung“ (Klimaschutz durch Ausdehnung der Waldfläche)}

Zusätzlich $\mathrm{zu}$ den in den vier Strategien zusammengefassten waldbaulichen Maßnahmenbündeln wurden zwei Maßnahmen als separate Module untersucht, die sich isoliert von den genannten Maßnahmenbündeln gestalten lassen. Im ersten dieser Module geht es um die Auswirkungen einer Ausdehnung der Waldfläche, vor allem mit Blick auf die Kohlenstoffsequestrierung. Dazu wurde eine Mehrung der Waldfläche um $10 \%$ unterstellt, der besseren Illustration halber ausschließlich mit Douglasien. Im zweiten Modul geht es um den Verzicht einer Nutzung des Waldes.

Die finanziellen Auswirkungen einer solchen Aufforstung sind dadurch gekennzeichnet, dass die neubegründeten Waldflächen hohe Anfangsinvestitionen aufwerfen, denen zunächst (im ersten 5-Jahres-Zeitraum) noch keine Erlöse gegenüberstehen. Rechnet man das Modul „Aufforstung“ der Klimaschutzstrategie zu und unterstellt man, dass die Kosten der Aufforstung von den forstlichen (Modell-)Betrieben selbst getragen werden müssen, dann schlagen sich diese Kosten im gesamtbetrieblichen Deckungsbeitrag der Klimaschutzstrategie nieder, der zu Beginn des Betrachtungszeitraumes um ca. $80 €$ je ha geringer ausfällt als ohne die Aufforstung (vgl. Abb. 4.44). Nach der 


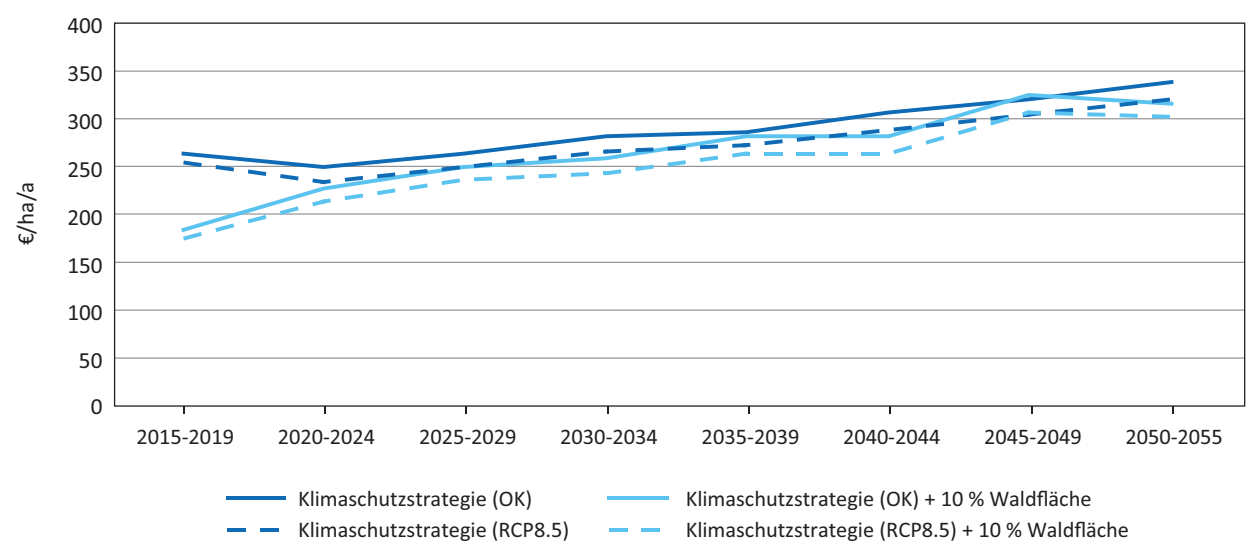

Abb. 4.44 Entwicklung des Deckungsbeitrages I in Deutschland bei einer Ausdehnung der Waldfläche um $10 \%$ in der Klimaschutzstrategie (2015-2055)

ersten 5-Jahres-Periode nehmen die Deckungsbeiträge auf den neubegründeten Flächen zwar zu, fallen jedoch weiterhin unterdurchschnittlich aus, sodass der durchschnittliche Deckungsbeitrag der um $10 \%$ vergrößerten gesamten deutschen Waldfläche über einen langen Zeitraum vermindert bleibt. Der Unterschied nivelliert sich erst zum Ende des Betrachtungszeitraumes, da die neubegründeten Douglasienflächen ein Alter erreichen, in dem Vornutzungserträge in nennenswerter Höhe generiert werden.

Im Durchschnitt des gesamten Betrachtungszeitraums entlastet das Modul „Aufforstung“ die Atmosphäre um zusätzliche 4,1 Mio. t C pro Jahr. Etwa die Hälfte dieses Betrages geht auf die Speicherveränderung in der ober- und unterirdischen Biomasse zurück (2,0 Mio. tC/a), weitere wesentliche Anteile entfallen auf die materielle und die energetische Substitution (1,1 bzw. 0,6 Mio. tC/a). Die Vergrößerung des Totholz- sowie des Harvested-Wood-Products-Speichers (HWP-Speicher) tragen lediglich 0,2 bzw. 0,1 Mio. t C/a bei.

Rechnet man diese Wirkungen der Klimaschutzstrategie zu, so steigt die durch sie bewirkte Entlastung der Atmosphäre dadurch von durchschnittlich 123,5 auf durchschnittlich 127,6 Mio. tC pro Jahr. Diese Entlastung verteilt sich ungleich über den Betrachtungszeitraum. Im Jahr 2015 beträgt sie 49,6 Mio. tC/a und steigt bis 2055 nahezu linear auf 221,2 Mio.tC/a an (ohne Modul „Aufforstung“ nur auf 213,4 Mio. tC/a). Zudem verschieben sich auch die Anteile, die auf die einzelnen Speicherkompartimente einerseits sowie die Substitution andererseits entfallen: Gehen in der ersten 5-JahresPeriode des Betrachtungszeitraums noch $55 \%$ der gesamten THG-Wirkung auf die Speicherveränderungen in Baumbiomasse, Totholz- und Holzprodukten zurück, so sinkt dieser Anteil bis 2055 auf lediglich $4 \%$ ab (ohne das Modul „Aufforstung“ sogar auf $2 \%$ ); alles Übrige entfällt auf die materielle und energetische Substitution. 


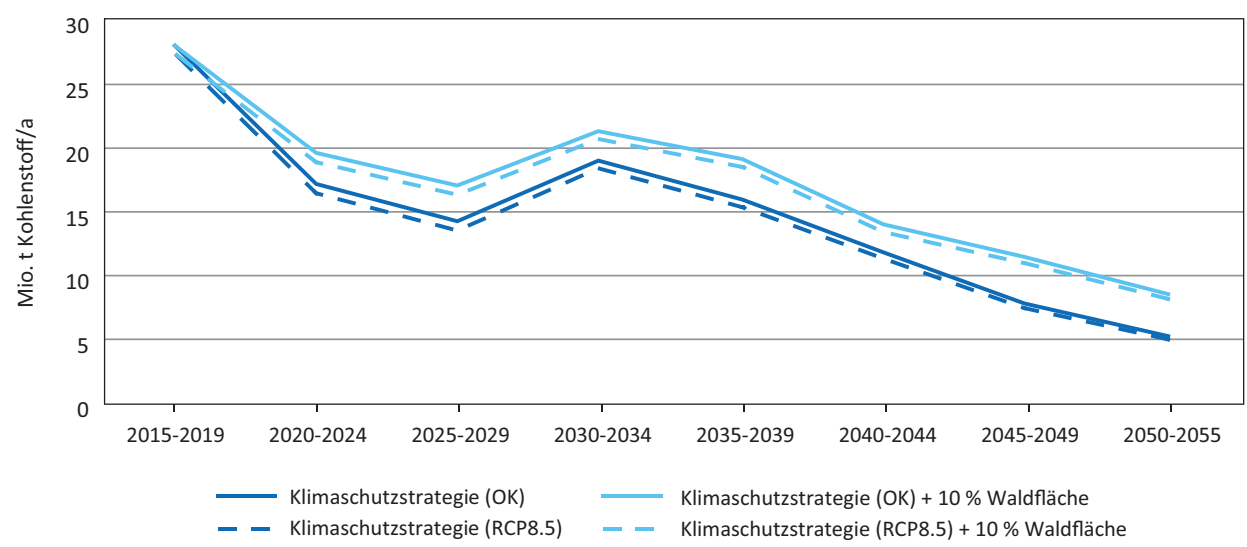

Abb.4.45 Entwicklung der Kohlenstoffsequestrierung in Baumbiomasse, Totholz und Holzproduktespeicher in Deutschland bei einer Ausdehnung der Waldfläche um $10 \%$ in der Klimaschutzstrategie (2015-2055; hier: ohne Substitution)

Dennoch lohnt sich auch ein Blick auf die zeitliche Entwicklung der durch die Ausdehnung der Waldfläche erzielbaren Kohlenstoffsequestrierung in lebender Baumbiomasse, Totholz- und Holzproduktespeicher allein. Sie ist (zu Illustrationszwecken ohne Berücksichtigung der Substitutionsleistung) in Abb. 4.45 dargestellt, wiederum am Beispiel der Klimaschutzstrategie. Durch die Waldflächenmehrung wird der im Zeitverlauf insgesamt abnehmenden Senkenleistung in den Wald- und Holzproduktspeichern Deutschlands entgegengewirkt, die durch die Altersstruktur des Waldes bedingt ist (vgl. Abschn. 1.2.4). Die neubegründeten Waldflächen weisen über den gesamten Betrachtungszeitraum eine zunehmende Senkenleistung auf, die ihr maximales Kohlenstoffsequestrierungspotenzial voraussichtlich erst nach 2055 erreichen werden.

\section{Modul ,Nutzungsverzicht“ (Klimaschutz durch Nutzungsverzichte im Wald)}

Der Verzicht einer Nutzung des Waldes gehört nicht zu den klassischen waldbaulichen Maßnahmen; vielmehr dient er Zielen, die von Seiten des Natur- und Umweltschutzes gefordert werden. Die Auswirkungen eines Verzichtes auf Waldnutzung lassen sich isoliert von sonstigen waldbaulichen Maßnahmen untersuchen. Für die Analyse wird ein Verzicht auf forstliche Nutzung auf einer Waldfläche von $10 \%$ unterstellt, der systematisch über alle Baumarten und Altersklassen verteilt wird.

Die finanziellen Auswirkungen dieses Nutzungsverzichtes gehen im Wesentlichen darauf zurück, dass auf gleichbleibender Fläche nur noch $90 \%$ der Erträge erwirtschaftet werden. Rechnet man das Modul „Nutzungsverzicht“ der Natur- und Umweltschutzstrategie zu, so verringert sich der durchschnittliche Deckungsbeitrag in dieser Strategie von Beginn an um den genannten Prozentsatz (vgl. Abb. 4.46). 


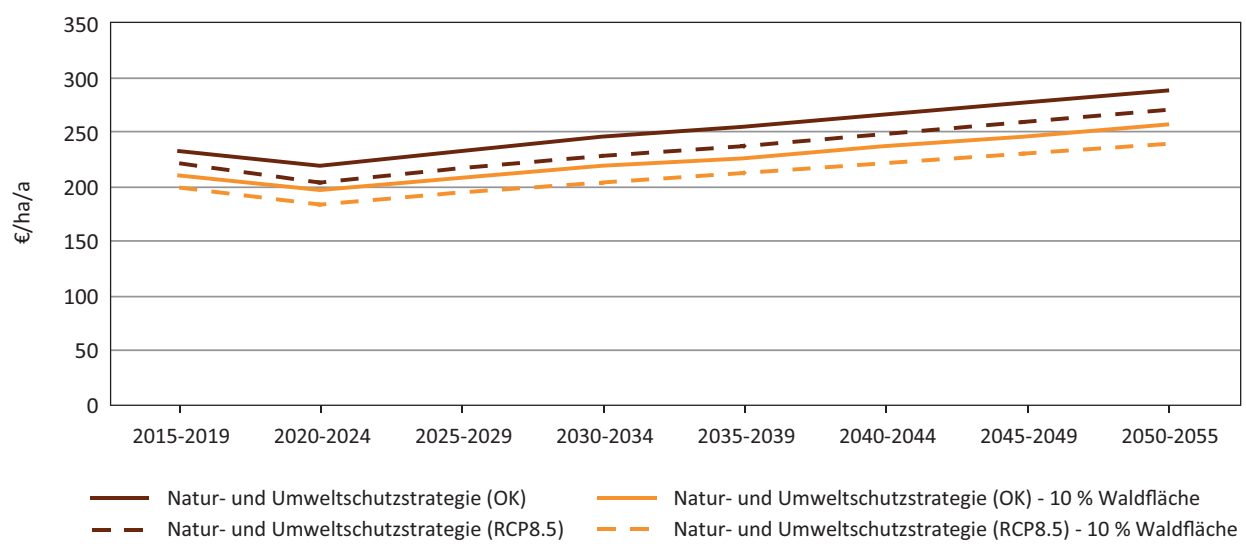

Abb. 4.46 Entwicklung des Deckungsbeitrages I bei einem Verzicht der Nutzung auf $10 \%$ der Waldfläche in Deutschland in der Natur- und Umweltschutzstrategie (2015-2055)

Die Kohlenstoffsequestrierung in der Baumbiomasse wird durch eine Waldflächenstilllegung erwartungsgemäß erhöht, da auf $10 \%$ der Flächen keine Holzernte mehr stattfindet. Das Speicherkompartiment „Totholz“ weist während des Betrachtungszeitraums eine nur leicht steigende Tendenz auf. Hierfür verantwortlich sind zwei gegenläufige Effekte, die sich im Modell weitgehend kompensieren: Zwar bewirkt der Nutzungsverzicht auch einen erhöhten Totholzanfall, andererseits entfällt auf den stillgelegten Flächen aber die Nachlieferung von Totholz durch Einschlagsrückstände. ${ }^{15}$ Der positiven Entwicklung des Baumbiomasse- und des Totholzspeichers steht gegenüber, dass aus den stillgelegten Flächen kein Zufluss zum Holzproduktespeicher mehr erfolgt; der Aufbau dieses Speichers wird daher um $10 \%$ vermindert. Gleichfalls entfallen alle Beiträge der stillgelegten Flächen zur materiellen und energetischen Substitution.

Quantitativ bedeutet dies, dass während des Betrachtungszeitraums durch den Nutzungsverzicht in den Speicherkompartimenten Baumbiomasse, Totholz und Holzprodukte insgesamt ein Speicheraufbau, also eine Sequestrierung, erfolgt. Im Beispiel der Natur- und Umweltschutzstrategie steigt diese Sequestrierung von 1,0 Mio. tC/a in der ersten 5-Jahres-Periode auf 2,5 Mio. tC/a in der letzten Periode an und beträgt im Durchschnitt des Betrachtungszeitraums 1,6 Mio. tC pro Jahr. Dem steht aber ein Verzicht auf Substitutionspotenziale i. H. v. durchschnittlich etwa 10 Mio. tC/a gegen-

\footnotetext{
${ }^{15} \mathrm{Es}$ ist zu erwarten, dass diese weitgehende gegenseitige Kompensation in ferner Zukunft ein Ende findet, wenn die Bäume auf den stillgelegten Flächen ein deutlich höheres Alter erreichen, da sich dann der Totholzanfall auf diesen Flächen erhöht - in diesem Fall zu Lasten des Baumbiomasse-Speichers. Generell sollte die Simulation der Totholzentwicklung aber nicht überinterpretiert werden, da das hier verwendete Modell nicht explizit auf eine Simulation von ,nicht genutzten Flächen“, also einem Baumwachstum deutlich über den forstüblichen Umtriebszeiten, ausgelegt ist.
} 
über. ${ }^{16}$ Betrachtet man daher den Klimaeffekt insgesamt einschließlich Substitution, so überwiegt per Saldo der Verzicht auf Substitutionspotenziale deutlich: Während die Natur- und Umweltschutzstrategie ohne Nutzungsverzichte im Durchschnitt eine Kohlenstoff-Einsparung von 122,8 Mio. tC/a erbringt, sinkt diese Einsparung durch die Nutzungsverzichte auf 113,7 Mio. tC/a, also um 9,1 Mio. tC jährlich.

\subsection{Ergebnisse der Modellierung von Stoffflüssen im Forst}

Martin Gutsch, Petra Lasch-Born und Felicitas Suckow

\section{Zusammenfassung}

Ziel der hier vorgestellten Modellergebnisse ist es, klimatische und bewirtschaftungsbezogene Auswirkungen auf die Kohlenstoff- und Wasserflüsse in den Wäldern bis zum Jahr 2050 zu untersuchen. Dazu wurde ein prozessbasiertes Waldwachstumsmodell mit den vorgestellten Klimaszenarien und Landnutzungsstrategien angewendet. Im Mittel (2011-2050) entwickeln sich die jährlichen Kohlenstoffflüsse unter der Referenzprojektion und -bewirtschaftung für den gesamten Wald wie folgt: Der in der gesamten Biomasse gespeicherte Kohlenstoff nimmt pro Jahr leicht ab $(-0,23 \mathrm{t} \mathrm{C} \mathrm{ha-1),} \mathrm{jedes} \mathrm{Jahr}$ werden 1,95 $\mathrm{t} \mathrm{C} \mathrm{ha}^{-1}$ geerntet, 1,44 $\mathrm{tC} \mathrm{ha}^{-1}$ im Boden gespeichert und 0,43 $\mathrm{t} \mathrm{C} \mathrm{ha}^{-1} \mathrm{im}$ Totholz. Demgegenüber steigt der Kohlenstoffvorrat in der Natur- und Umweltschutzstrategie aufgrund geringerer Holzerntemengen $\left(1,71 \mathrm{t} \mathrm{Cha}^{-1}\right)$ leicht an. Besonders stark ist der Rückgang des Kohlenstoffvorrats in der Bioenergiestrategie und etwa gleichbleibend in der Klimaschutzstrategie. Das Klimaszenario „RCP 8.5“ führt im Mittel bis $2050 \mathrm{zu}$ positiven Waldwachstumseffekten, die sich in höheren biomasse- und erntebezogenen Kohlenstoffflüssen ausdrücken. Die Kohlenstoffspeicherung des Bodens geht unter diesem Klimaszenario um etwa $8 \%$ zurück, führt zu einer erhöhten Verdunstung und damit zu geringeren Versickerungsraten im Vergleich zur Referenzprojektion. Der Einfluss der Bewirtschaftungsstrategien auf die Wasserflüsse ist im Mittel über die Gesamtwaldfläche sehr gering. Diese Effekte werden erst auf der Skala von Waldbeständen und Landschaften sichtbar.

Die Simulation von Waldbeständen sowie Stoffflüssen im Forst erfolgte mit dem Modell 4C (vgl. Abschn. 4.2.2.5) von 2011-2050 für die Referenzbewirtschaftung, den vier Landnutzungsstrategien „Klimaschutz“, „Bioenergie“, „Natur- und Umweltschutz“ sowie „Klimaanpassung“ und den zwei Klimaszenarien „,K-Referenzprojektion“ und

\footnotetext{
${ }^{16} \mathrm{Im}$ Modul „Nutzungsverzichte“ haben Substitutionspotenziale einen größeren Anteil an der gesamten Kohlenstoffeinsparung als im Modul „Aufforstung“. Dies ist plausibel, da Aufforstungsflächen in ihrer Jugend vergleichsweise weniger nutzbares Holz produzieren (welches andere Materialien substituieren könnte), und der hier berücksichtigte Betrachtungszeitraum von 40 Jahren lediglich die Jugendphase der aufgeforsteten Bestände umfasst.
} 
„RCP 8.5-Standardszenario“ (vgl. Abschn. 4.3). Im Unterschied zu den Simulationen in Abschn. 4.6 wurde modellbedingt der akkumulierte Kohlenstoff in der Ausgangssituation (Vorperiode) berücksichtigt, nicht aber der Holzproduktespeicher (HWP), sodass die Ergebnisse nur eingeschränkt mit den in Abschn. 4.6 dargestellten Ergebnissen vergleichbar sind.

Die Maßnahmen der strategiespezifischen Bewirtschaftungsbündel wurden ebenfalls baumarten-, altersklassen- und bundeslandspezifisch in den Simulationen berücksichtigt. Daher ergab sich bei 40 Jahren Simulationszeit ein Altersklassenwechsel (eine Altersklasse umfasst 20 Jahre) mit entsprechenden Wechseln in den Maßnahmen. Der Anteil des Nutzungsverzichts beruht auf den Ergebnissen der Studie zum Stand der Flächenausweisung von Beständen mit natürlicher Waldentwicklung (vgl. Abschn. 4.4). Dabei wurde über alle Bestände eines Landkreises der strategiespezifische Anteil an Beständen ohne forstliche Eingriffe zufällig ausgewählt. Im Unterschied dazu wurden die prozentualen Vorgaben (vgl. Tab. 4.21) für einen Baumartenwechsel nur auf Beständen mit erfolgter Endnutzung realisiert.

Die Implementierung des Aufforstungsszenarios erfolgte mit den Ergebnisdaten des LUS (vgl. Abschn. 4.4). Aus diesen Daten wurde eine kreisspezifische jährliche Veränderungsrate der Waldfläche bis 2030 (ab 2030 keine Waldflächenänderung) berechnet und umgesetzt. Die so ermittelte Waldfläche wurde mit den kreis- und baumartenspezifischen 4C-Ergebnissen der Altersklassen 10-30 verschnitten, um die stoffflussbezogenen Analysen durchzuführen.

\subsubsection{Ergebnisse der Referenzprojektion}

Die Maßnahmenbündel in der Referenzprojektion und der vier forstlichen Bewirtschaftungsstrategien (vgl. Abschn. 4.6) wurden für die Analyse der Stoffflüsse mit dem Waldwachstumsmodell 4C angepasst, um eine größtmögliche Vergleichbarkeit der Simulationsergebnisse zu den Simulationen mit dem Modell FoBeSiMo zu gewährleisten. Die Maßnahmen „Bestandesbegründung“, „Durchforstungsart“ und „Nutzungsverzicht“ wurden analog zu FoBeSiMo umgesetzt. Die Maßnahmen „Z-Baumauswahl“, „Zielstärke“ und „Holzbereitstellung“ blieben in 4C unberücksichtigt. Die forstlichen Eingriffe wurden mit 4C jährlich simuliert, wobei der Durchforstungsbeginn im zweiten Jahr der Simulation angesetzt ist. Die strategiespezifischen prozentualen Unterschiede in der Durchforstungsstärke wurden durch verschiedene Nutzungsprozente realisiert. Der Endnutzungszeitraum wurde auf Basis der in Tab. 4.21 festgelegten strategiebezogenen Umtriebszeiten bundes- und baumartenspezifisch implementiert (vgl. Tab. 4.25) und in Form eines Kahlschlages mit einem Eingriff umgesetzt. Danach fand die strategiespezifische Bestandesbegründung in Form einer Pflanzung mit 2000 Pflanzen pro Hektar statt.

Die Bewirtschaftung in der Referenzprojektion bewirkt, gemittelt über alle betrachteten Waldbestände, für den Zeitraum 2011-2050 eine positive jährliche Kohlenstoffspeicherung (C-Speicherung) im Boden (C-Boden), im Totholz und im geernteten 


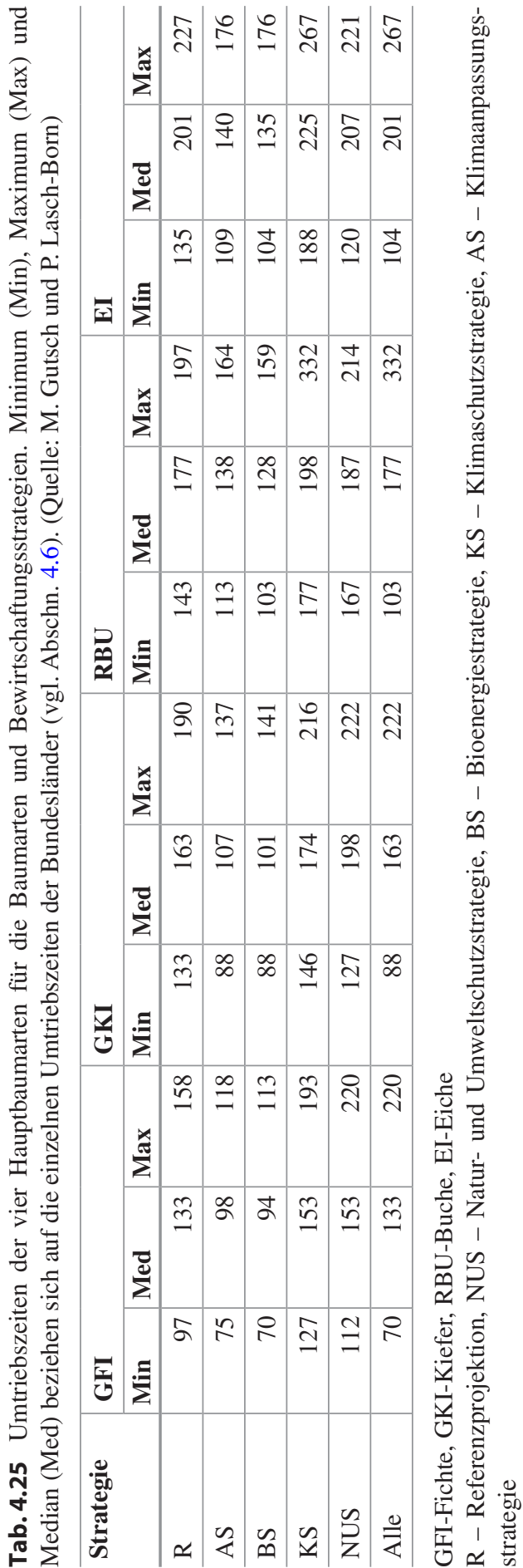


Holz (Ernte). Im Gegensatz dazu geht die C-Speicherung in der ober- und unterirdischen Biomasse (Biomasse; vgl. Abb. 4.47) bei den Baumarten Buche und Fichte zurück. Dies bedeutet, dass am Ende des Betrachtungszeitraums (2050) weniger Kohlenstoff in der Gesamtbiomasse in der Summe aller Buchen- und Fichtenbestände gespeichert ist als am Anfang. Die C-Speicherungen unter dem Klimaszenario „RCP 8.5“ unterscheiden sich nur geringfügig von denen für das 0K-Szenario, wenn man die Mittelwerte über alle Bestände betrachtet. Die C-Speicherung im Boden ist unter dem Szenario „RCP 8.5“ leicht rückgängig, die Speicherung in der Biomasse dagegen steigt leicht an (vgl. Abb. 4.47).

Die jährliche Verdunstungsrate steigt im Vergleich zum 0K-Szenario unter dem RCP 8.5-Szenario sowohl im Mittel über alle Baumarten als auch für jede einzelne Baumart, was vor allem auf die höheren Temperaturen zurückzuführen ist. Dagegen geht die Versickerungsrate unter dem RCP 8.5-Szenario für alle Baumarten zurück. Im

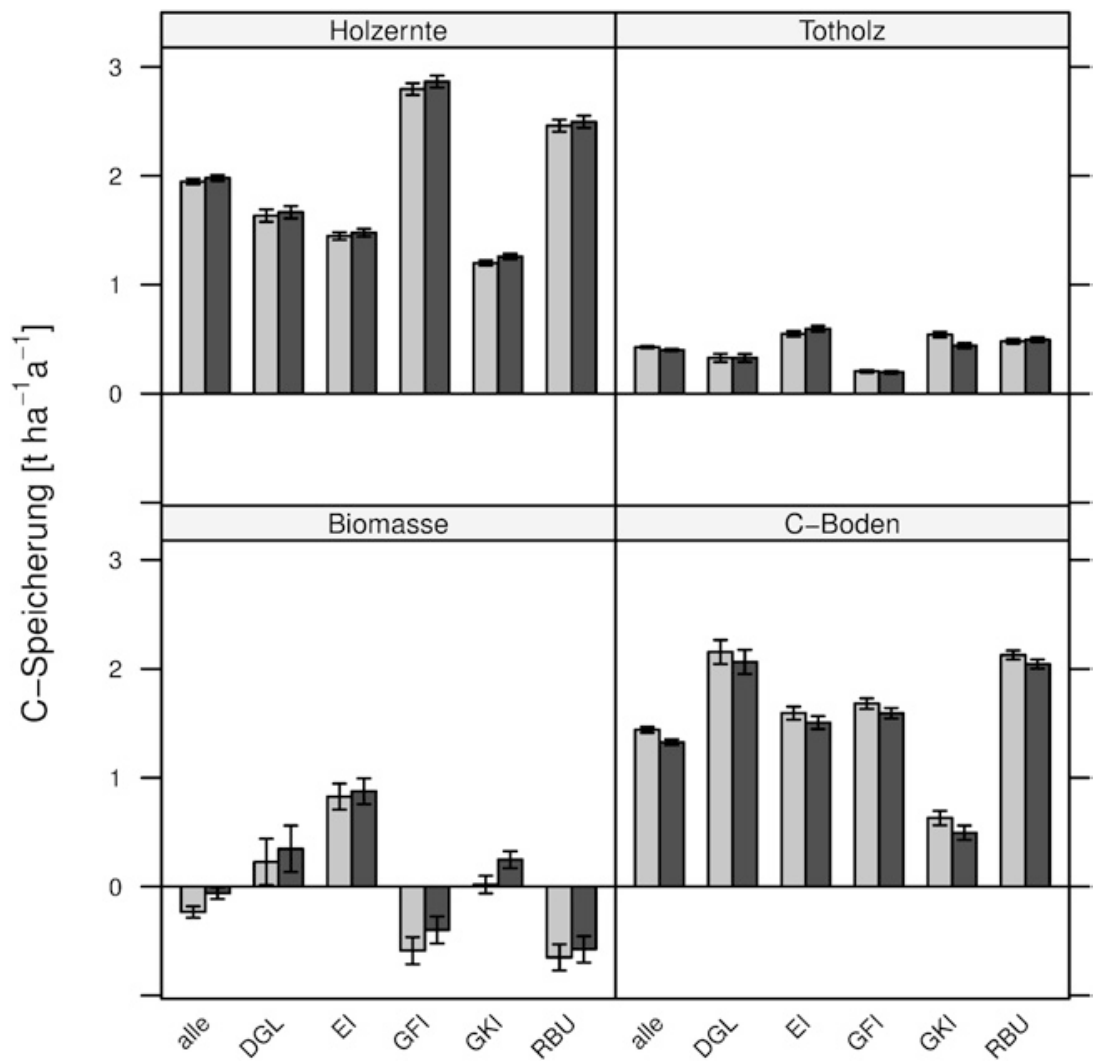

Abb. 4.47 Mittlere jährliche C-Speicherung unter der Referenzbewirtschaftung und den Klimaszenarien „,OK“ (hell) und „RCP 8.5“ (dunkel) für alle Wälder und getrennt nach Baumarten (DGL- Douglasie, Ei- Eiche, GFI- Fichte, GKI- Kiefer, RBU- Buche). Die Whisker stellen die Varianz der Mittelwerte dar 


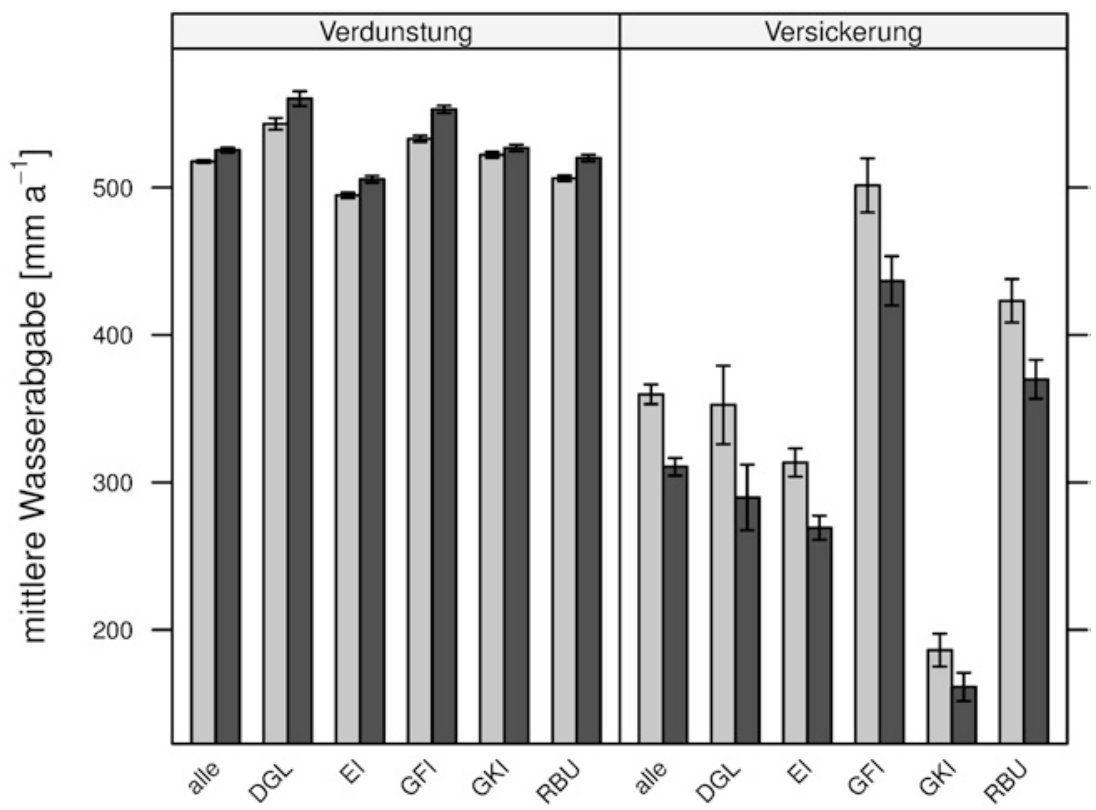

Abb.4.48 Mittlere jährliche Verdunstung und Versickerung für 2011-2050 aller Waldbestände unter der Referenzbewirtschaftung und für die Klimaszenarien „OK“ (hell) und „RCP 8.5“ (dunkel) und getrennt nach Baumarten (DGL- Douglasie, Ei-Eiche, GFI- Fichte, GKI- Kiefer, RBU- Buche). Die Whisker stellen die Varianz der Mittelwerte dar

Vergleich der beiden Klimaszenarien nimmt die Verdunstung unter dem „RCP 8.5“ nur leicht zu, während die Versickerung stärker abnimmt (vgl. Abb. 4.48). Die Variabilität der Verdunstung und der Versickerung zwischen den Baumarten ist insbesondere durch die Standortvariabilität bedingt, da beispielsweise die Mehrzahl der Fichtenstandorte in Gebirgsregionen mit relativ hohem Niederschlag liegen, während Kiefernbestände auf Standorten mit geringen Niederschlägen dominieren.

\subsubsection{Ergebnisse der Landnutzungsstrategien}

\subsubsection{Klimaschutzstrategie}

Die Klimaschutzstrategie führt im Vergleich zur Bewirtschaftung in der Referenzprojektion nur zu einer geringen Änderung der Kohlenstoffflüsse in die untersuchten Kohlenstoffpools „,ober- und unterirdische Biomasse“, „Boden“, „Ernteholz“ und „Totholz“ (Abb. 4.49). Das gilt auch für die betrachteten Wasserflüsse, die im Mittel über alle Waldbestände unter der Klimaschutzstrategie nur geringfügig von der Referenzprojektion abweichen. Für beide Bewirtschaftungen hat das Klimaszenario einen wesentlich deutlicheren Effekt auf die Verdunstung, aber vor allem auf die Versickerung. Diese geht unter 
Abb. 4.49 Änderung in der mittleren jährlichen C-Speicherung unter der Klimaschutzstrategie im Vergleich zur Referenzprojektion im Zeitraum 2011-2050 unter den Klimaszenarien „,0K“ (hell) und dem „RCP 8.5“ (dunkel). Die Whisker stellen die Varianz der Mittelwerte dar

Abb. 4.50 Änderung der mittleren jährlichen Verdunstung und Versickerung aller Waldbestände unter der Klimaschutzstrategie im Vergleich zur Referenzprojektion unter den Klimaszenarien „,0K“ (hell) und „RCP 8.5“ (dunkel). Die Whisker stellen die Varianz der Mittelwerte dar
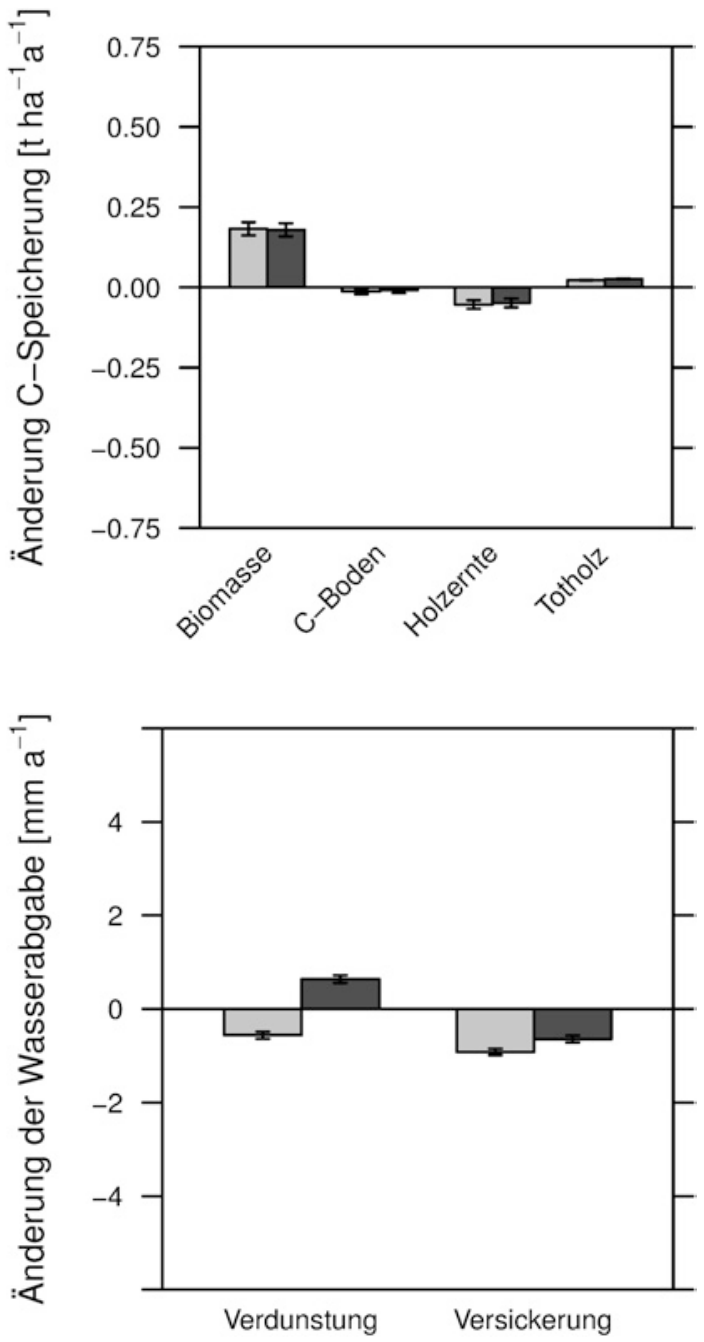

dem „RCP 8.5“ - sowohl unter der Referenzprojektion als auch unter der Klimaschutzstrategie - im Vergleich zum 0K-Szenario um circa $14 \%$ zurück (Abb. 4.49 und 4.50).

\subsubsection{Bioenergiestrategie}

Der Fokus auf mehr Biomasse für die Energiegewinnung führt im Vergleich zur Referenzprojektion zu einer im Mittel über alle Waldbestände höheren jährlichen Ernte, aber zu einer geringeren C-Speicherung in der Biomasse (vgl. Abb. 4.51).

Durch die Bioenergiestrategie nimmt die Verdunstungsrate im Mittel im Vergleich zur Referenzbewirtschaftung leicht ab und die Versickerungsrate leicht $\mathrm{zu}$, dies ist vor allem auf die geänderte Bestandsstruktur durch höhere Ernten zurückzuführen (Abb. 4.52). 
Abb. 4.51 Änderungen der jährlichen mittleren C-Speicherung unter der Bioenergiestrategie im Vergleich zur Referenzprojektion für 20112050 unter den Klimaszenarien „OK“ (hell) und „RCP 8.5“ (dunkel). Die Whisker stellen die Varianz der Mittelwerte dar

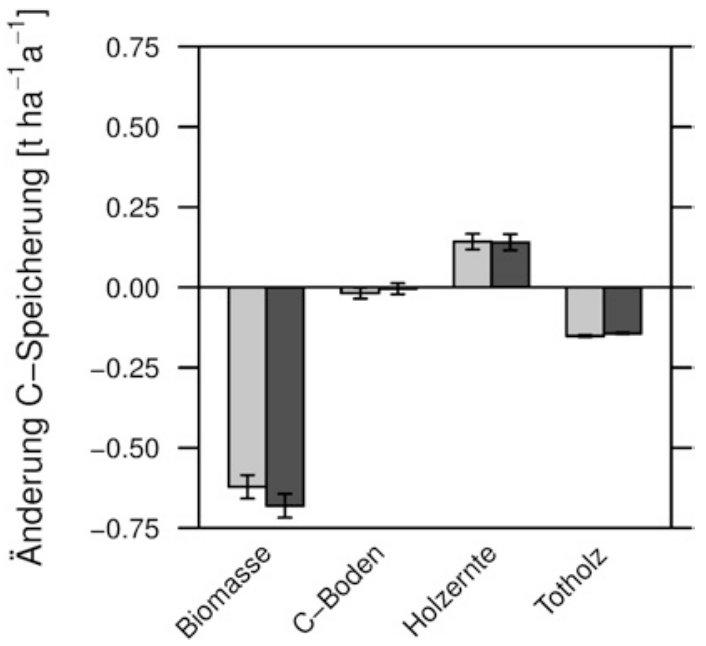

Abb. 4.52 Änderung der mittleren jährlichen Verdunstung und Versickerung aller Waldbestände für 2011-2050 unter der Bioenergiestrategie im Vergleich zur Referenzprojektion und unter den Klimaszenarien „, $0 \mathrm{~K}^{\prime \prime}$ (hell) und „RCP 8.5“ (dunkel). Die Whisker stellen die Varianz der Mittelwerte dar

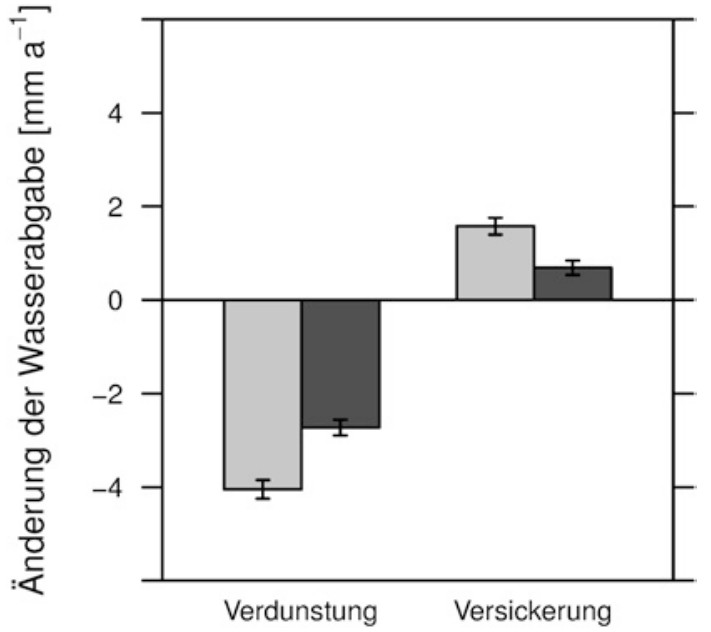

Das RCP 8.5-Szenario bewirkt eine etwas geringere mittlere jährliche C-Speicherung in der Biomasse im Vergleich zum 0K-Szenario und eine etwas höhere Verdunstungssowie eine geringere Versickerungsrate.

\subsubsection{Natur- und Umweltschutzstrategie}

Die Natur- und Umweltschutzstrategie führt im Mittel über alle Wälder zu einer Erhöhung der jährlichen C-Speicherung in den Wäldern (in der ober- und unterirdischen Biomasse und im Totholz) gegenüber der Referenzprojektion (Abb. 4.53). Im Mittel gehen durch die höhere Biomasse der Wälder die jährlichen Versickerungsraten im Vergleich zur Referenzprojektion zurück und die Verdunstungsraten steigen (vgl. Abb. 4.53). 
Abb. 4.53 Änderungen der jährlichen mittleren C-Speicherung unter der Naturund Umweltschutzstrategie im Vergleich zur Referenzprojektion für 20112050 unter den Klimaszenarien „OK“ (hell) und „RCP 8.5“ (dunkel). Die Whisker stellen die Varianz der Mittelwerte dar

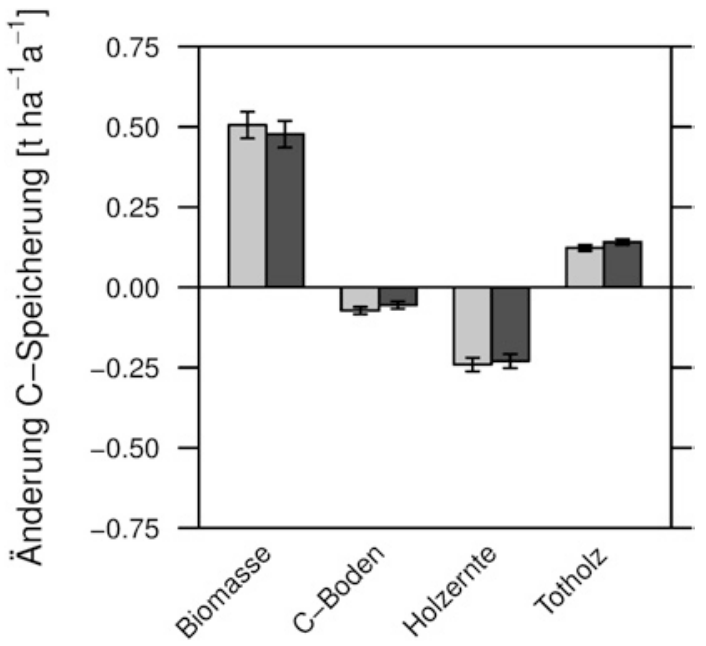

Abb. 4.54 Änderung der mittleren jährlichen Verdunstung und Versickerung aller Waldbestände für 2011-2050 unter der Naturund Umweltschutzstrategie im Vergleich zur

Referenzprojektion und unter den Klimaszenarien „,OK“ (hell) und „RCP 8.5“ (dunkel). Die Whisker stellen die Varianz der Mittelwerte dar

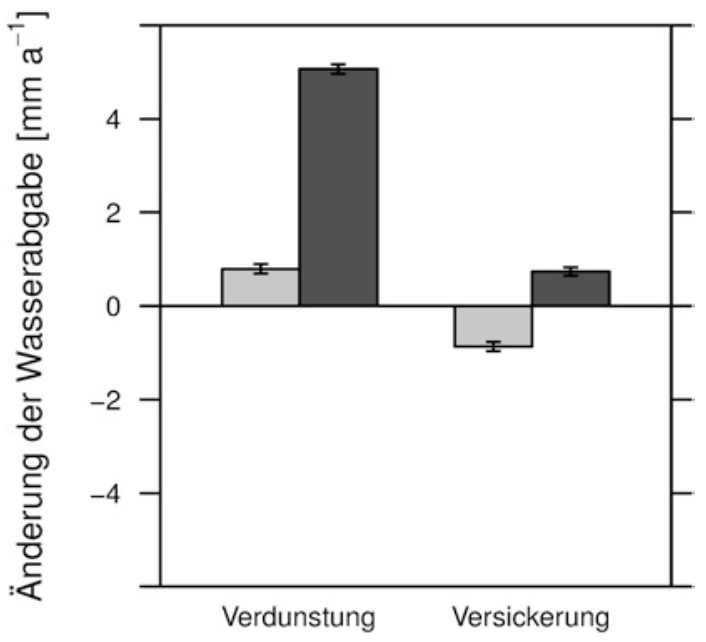

Deutliche Unterschiede der C-Speicherungsraten unter dem 0K- und dem RCP 8.5-Szenario sind nicht festzustellen. Die jährlichen Verdunstungsraten sind unter dem RCP 8.5-Szenario aber höher als unter dem 0K-Szenario aufgrund der höheren Temperaturen und des damit verbundenen höheren Verdunstungsanspruchs der Atmosphäre (Abb. 4.54).

\subsubsection{Klimaanpassungsstrategie}

Die mit 4C simulierte Klimaanpassungsstrategie bewirkt bezüglich der Kohlenstoffflüsse, gemittelt über alle simulierten Waldbestände, ähnliche Veränderungen im Vergleich zur Referenzprojektion wie die Strategie „Bioenergie“. Die C-Speicherung in der Biomasse 
Abb. 4.55 Änderungen der jährlichen mittleren C-Speicherungen unter der Klimaanpassungsstrategie im Vergleich zur Referenzprojektion für 20112050 unter den Klimaszenarien „0K“ (hell) und „RCP 8.5“ (dunkel). Die Whisker stellen die Varianz der Mittelwerte dar

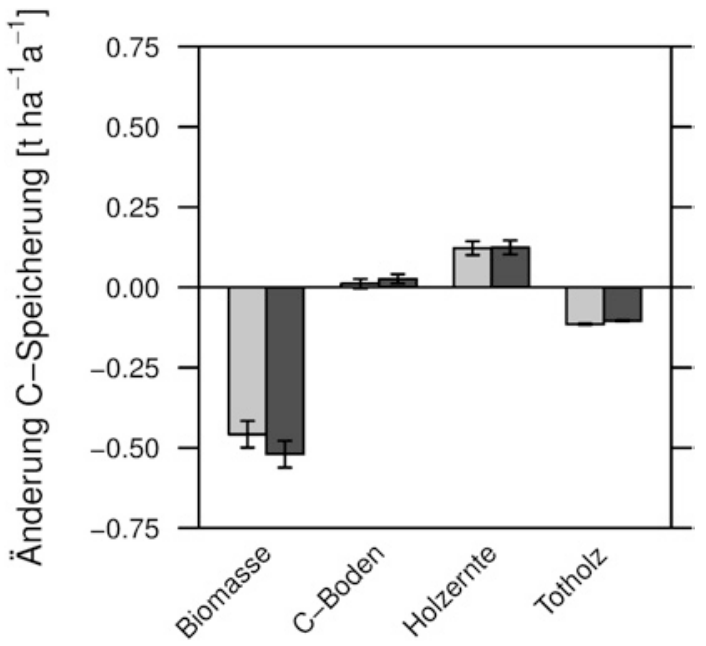

Abb. 4.56 Änderung der mittleren jährlichen Verdunstung und Versickerung aller Waldbestände für 2011-2050 unter der Klimaanpassungsstrategie im Vergleich zur Referenzprojektion und unter den Klimaszenarien „,OK“ (hell) und „RCP 8.5“ (dunkel). Die Whisker stellen die Varianz der Mittelwerte dar

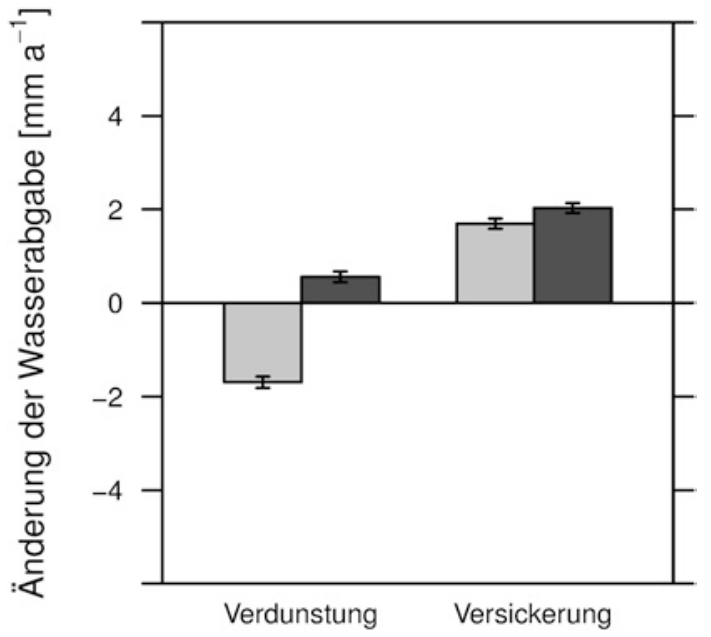

geht zurück, die im Ernteholz nimmt leicht zu. Diese Effekte sind unter beiden Klimaszenarien gleich (Abb. 4.55).

Die mittlere jährliche Verdunstungsrate ist bei der Klimaanpassungsstrategie etwas geringer als bei der Referenzprojektion und die Versickerungsrate nur minimal höher als unter der Referenzprojektion (vgl. Abb. 4.56). Auch hier führt das RCP 8.5-Szenario zu einer leicht höheren mittleren jährlichen Verdunstungsrate als das 0K-Szenario. 


\subsubsection{Vergleichende Beurteilung der Auswirkungen der Strategien}

Im Gegensatz zu den vorangegangenen Darstellungen der 4C-Ergebnisse (mittlere Änderungen zur Referenzprojektion) sind in der Tab. 4.26 die absoluten mittleren jährlichen Kohlenstoff- und Wasserflüsse im Simulationszeitraum gelistet. Der Vergleich der C-Speicherungen der Referenzprojektion und der vier Landnutzungsstrategien zeigt, dass die Natur- und Umweltschutzstrategie am deutlichsten von der Referenzprojektion abweicht, insbesondere bei der C-Speicherung im Totholz und in der Biomasse (vgl. Tab. 4.26). Vergleichsweise ähnlich der Referenzprojektion zeigen sich die Auswirkungen der Klimaschutzstrategie. Die Effekte der Bewirtschaftungsstrategien auf die Wasserflüsse sind im Vergleich zur Referenzprojektion im Mittel über alle Bestände sehr gering. Dieselben Aussagen treffen für die Flüsse unter dem Klimaszenario „RCP 8.5“ zu, unter dem aber die jährlichen mittleren C-Speicherungen für alle Strategien höher sind als unter dem 0K-Szenario. Die jährliche Verdunstungsrate ist im Mittel leicht erhöht gegenüber dem 0K-Szenario, während die Versickerungsrate deutlich geringer ist, unabhängig von der Bewirtschaftung (vgl. Tab. 4.26). Sie geht um circa 13 bis $14 \%$ zurück. 


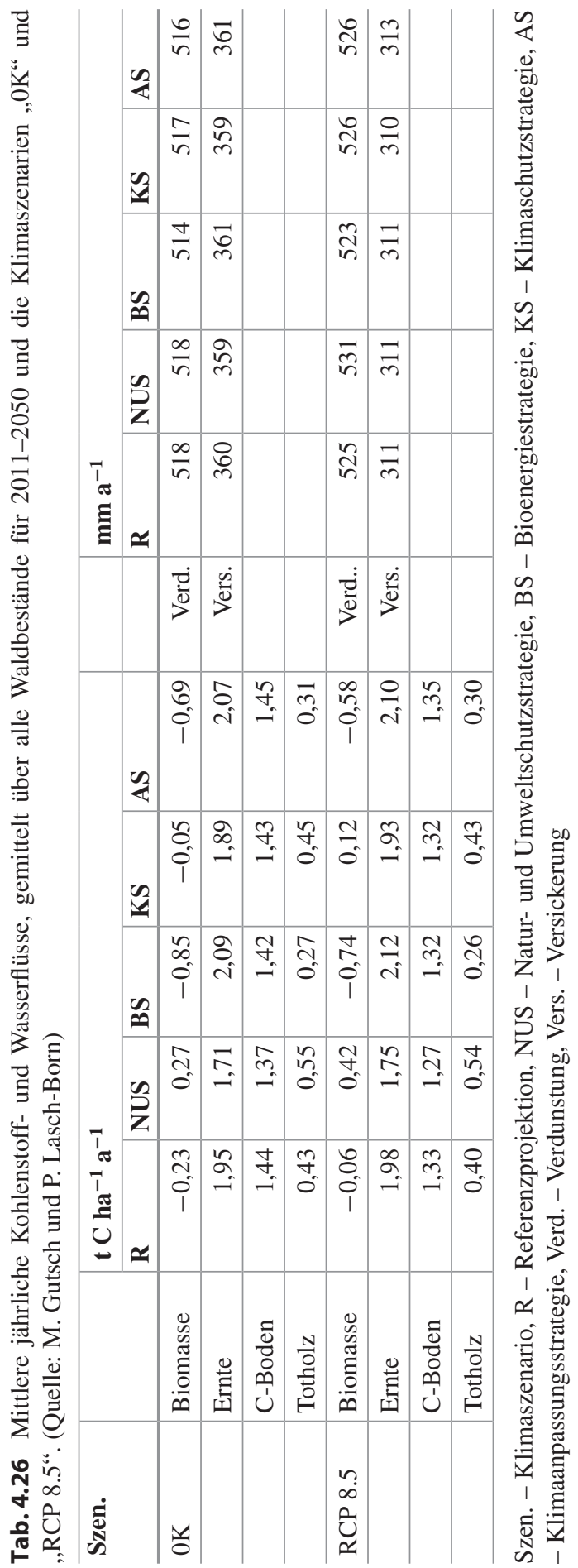




\section{Literatur}

Batista e Silva F, Lavalle C, Koomen E (2013) A procedure to obtain a refined European land use/ cover map. J Land Use Sci 3:255-283

Bechtold M, Tiemeyer B, Laggner A, Leppelt T, Frahm E, Belting S (2014) Large-scale regionalization of water table depth in peatlands optimized for greenhouse gas emission upscaling. Hydrol Earth Syst Sci 18(9):3319-3339

Bertelsmeier M, Gömann H, Kleinhanß W, Kreins P, Manegold D, Offermann F (2002) Modellanalysen zu den Auswirkungen der KOM-Vorschläge im Rahmen der Halbzeitbewertung der Agenda 2000. SchrR Forschungsgesellsch Agrarpol Agrarsoz 320:97

Biber et al (2000) SILVA 2.2. Benutzerhandbuch. Lehrstuhl für Waldwachstumskunde der Technischen Universität München, München

Bonn A, Berghöfer A, Couwenberg J, Drösler M, Jensen R, Kantelhardt J, Luthardt V, Permien T, Röder N, Schaller L, Schweppe-Kraft B, Tanneberger F, Trepel M, Wichmann S (2015) Klimaschutz durch Wiedervernässung von kohlenstoffreichen Böden. In: Hartje V, Wüstemann H, Bonn A (Hrsg) Naturkapital Deutschland - TEEB DE: Naturkapital und Klimapolitik - Synergien und Konflikte. Technische Universität Berlin \& Helmholtz-Zentrum für Umweltforschung - UFZ, Berlin \& Leipzig, S 124-147

Bundesanstalt für Geowissenschaften und Rohstoffe (BGR) (2004) Nutzungsdifferenzierte Bodenübersichtskarte der Bundesrepublik Deutschland 1:1.000.000 (Wald-BÜK 1000)

Bundesinstitut für Bau-, Stadt- und Raumforschung (BBSR) (2013) Innenentwicklungspotenziale in Deutschland. Ergebnisse einer bundesweiten Umfrage und Möglichkeiten einer automatisierten Abschätzung. Unter Mitarbeit von Schiller G, Blum A, Hecht R, Meinel G, Oertel H, Ferber U, Petermann E (Hrsg.), Bonn, S 163

Bundesinstitut für Bau-, Stadt- und Raumforschung (BBSR) (2015a) Raumordnungsprognose 2035 nach dem Zensus. BBSR-Analysen KOMPAKT 05/2015, Bonn

Bundesinstitut für Bau-, Stadt- und Raumforschung (BBSR) (2015b) Überflutungs- und Hitzevorsorge durch die Stadtentwicklung. Strategien und Maßnahmen zumRegenwassermanagement gegen urbane Sturzfluten und überhitzte Städte, Bonn, S 120

Bundesministerium für Ernährung und Landwirtschaft (BMEL) (2013) Die wirtschaftliche Lage der forstwirtschaftlichen Betriebe. Buchführungsergebnisse der Forsttestbetriebe, Forstwirtschaftsjahr 2013

Bundesministerium für Ernährung und Landwirtschaft (BMEL) (2014) Der Wald in Deutschland, Ausgewählte Ergebnisse der dritten Bundeswaldinventur. Bundesministerium für Ernährung und Landwirtschaft, Berlin

Bundesministerium für Ernährung, Landwirtschaft und Verbraucherschutz (BMELV) (2005) Die zweite Bundeswaldinventur. Der Inventurbericht. Bundesministerium für Ernährung, Landwirtschaft und Verbraucherschutz (BMELV), Bonn

Bundesministerium für Ernährung, Landwirtschaft und Verbraucherschutz (BMELV), Bundesministerium für Umwelt, Naturschutz und nukleare Sicherheit (BMU) (2010) Nationaler Biomasseaktionsplan für Deutschland - Beitrag der Biomassefür eine nachhaltige Energieversorgung. BMELV, Berlin

Bundesministerium für Umwelt, Naturschutz und nukleare Sicherheit (BMU) (Hrsg) (2012) Aktionsplan Anpassung der Deutschen Anpassungsstrategie an den Klimawandel. BMU, Berlin

Bundesministerium für Verkehr, Bau und Stadtentwicklung (BMVBS) (2011) 30-ha-Ziel realisiert - Konsequenzen des Szenarios Flächenverbrauchsreduktion auf 30 ha im Jahr 2020 für die Siedlungsentwicklung. Forschungen 148. Bundesinstitut für Bau-, Stadt- und Raumforschung (BBSR), Bonn, S 78 
Bundesministerium für Verkehr, Bau und Stadtentwicklung (BMVBS), Bundesamt für Bauwesen und Raumordnung (BBR) (2007) Nachhaltigkeitsbarometer Fläche. Regionale Schlüsselindikatoren nachhaltiger Flächennutzung für die Fortschrittsberichte der nationalen Nachhaltigkeitsstrategie; Flächenziele; ein Projekt des Forschungsprogramms „Allgemeine Ressortforschung“" des Bundesministeriums für Verkehr, Bau und Stadtentwicklung (BMVBS) und des Bundesamtes für Bauwesen und Raumordnung (BBR), [Elektronische Ressource]. BMVBS \& BBR, Berlin \& Bonn

Bundesregierung (2008) Deutsche Anpassungsstrategie an den Klimawandel vom Bundeskabinett am 17. Dezember 2008 beschlossen. https://www.bmub.bund.de/fileadmin/bmu-import/files/ pdfs/allgemein/application/pdf/das_gesamt_bf.pdf. Zugegriffen: 19. März 2019

Burkett VR, Suarez AG, Bindi M, Conde C, Mukerji R, Prather MJ, Clair ALS, Yohe GW (2014) Point of departure. In: Field CB, Barros VR, Dokken DJ, Mach KJ, Mastrandrea MD, Bilir TE, Chatterjee M, Ebi KL, Estrada YO, Genova RC, Girma B, Kissel ES, Levy AN, MacCracken S, Mastrandrea PR, White LL (Hrsg) Climate change 2014: impacts, adaptation, and vulnerability. Part A: global and sectoral aspects. Contribution of working group II to the fifth assessment report of the intergovernmental panel of climate change. Cambridge University Press, Cambridge, S 169-194

Collins M, Knutti R, Arblaster J, Dufresne J-L, Fichefet T, Friedlingstein P, Gao X, Gutowski WJ, Johns T, Krinner G, Shongwe M, Tebaldi C, Weaver AJ, Wehner M (2013) Long-term climate change: projections, commitments and irreversibility. In: Stocker TF, Qin D, Plattner GK, Tignor M, Allen SK, Boschung J, Nauels A, Xia Y, Bex V, Midgley PM (Hrsg) Climate change 2013: the physical science basis. Contribution of working group I to the fifth assessment report of the intergovernmental panel on climate change. Cambridge University Press, Cambridge, S 1029 1136

Cypris C (2000) Positive Mathematische Programmierung (PMP) im Agrarsektormodell RAUMIS. Schriftenreihe der Forschungsgesellschaft für Agrarpolitik und Agrarsoziologie e. V., Bonn

Cypris C, Kreins P (1998) Das Agrar- und Umweltinformationssystem RAUMIS - ein Prototyp für Analysen des Transformationsprozesses in den MOE-Ländern? In: Heißenhuber A, Hoffmann H, von Urff W (Hrsg) Land- Und Ernährungswirtschaft in Einer Erweiterten EU. Schriften der Gesellschaft für Wirtschafts- und Sozialwissenschaften des Landbaues e. V., Bd 34. Landwirtschaftsverlag, Münster-Hiltrup, S 53-61

Dechow R, Freibauer A (2011) Assessment of German nitrous oxide emissions using empirical modelling approaches. Nutr Cycl Agroecosyst 91(3):235-254

Delzeit R, Zabel F, Meyer C, Václavík T (2016) Addressing future trade-offs between biodiversity and cropland expansion to improve food security. Reg Environ Change 17(5):1429-1441

Delzeit R, Zabel F, Meyer C, Václavík T (2017) Addressing future trade-offs between biodiversity and cropland expansion to improve food security. Reg Environ Change 17:1429

Deutscher Wetterdienst (DWD) (2016) Mitteltemperaturen Deutschland 1881-2015. https://www. dwd.de/DE/leistungen/zeitreihenundtrends/zeitreihenundtrends.html?nn=495662. Zugegriffen: 25. Jan. 2016

Dieter M, Elsasser P, Wiehler HA (2005) Anrechnung der deutschen Waldbewirtschaftung in der Klimapolitik? Ein Diskussionsbeitrag zur Wahlmöglichkeit nach Artikel 3.4 des KyotoProtokolls. Forst Holz 60(11):472-474

DLR-DFD (2004) CORINE Land Cover (CLC2000)

Dodman D (2009) Blaming cities for climate change? An analysis of urban greenhouse gas emission inventories. Environ Urbani 21(1):85-201

Drösler M, Freibauer A, Adelmann W, Augustin J, Bergman L, Beyer C, Chojnicki B, Förster C, Giebels M, Görlitz S, Höper H, Kantelhardt J, Liebersbach H, Hahn-Schöfl M, Minke M, Petschow U, Pfadenhauer J, Schaller L, Schägner P, Sommer M, Thuille A, Wehrhan M (2013) 
Klimaschutz durch Moorschutz. Schlussbericht des BMBF-Vorhabens: Klimaschutz-Moornutzungsstrategien 2006-2010

Ermisch N, Seintsch B, Dög M (2014) Ergebnisse des Testbetriebsnetzes Forst des BMEL Das Jahr 2012 folgte dem positiven Trend der Vorjahre: Die drei Eigentumsarten des TBN-Forst erwirtschafteten ein positives Betriebsergebnis. AFZ-DerWald 23:18-20

Fick J, Baum S, Dechow R, Elsasser P, Ermisch N, Goetzke R, Gömann H, Grabski-Kieron U, Gutsch M, Hellmich M, Henseler M, Hirschfeld J, Hoymann J, Kreins P, Lasch-Born P, Raabe M, Sagebiel J, Siebert R, Steinführer A, Steinhäußer R, Wechsung F, Weingarten P, Weller P, Gömann H (2016) Interdependencies between land use and climate changestrategies for a sustainable land use management in Germany(CC-LandStraD). Unpublished final report, Braunschweig

Flato G, Marotzke J, Abiodun B, Braconnot P, Chou SC, Collins W, Cox P, Driouech F, Emori S, Eyring V, Forest C, Gleckler P, Guilyardi E, Jakob C, Kattsov V, Reason C, Rummukainen M (2013) Evaluation of climate models. In: Stocker TF, Qin D, Plattner GK, Tignor M, Allen SK, Boschung J, Nauels A, Xia Y, Bex V, Midgley PM (Hrsg) Climate change 2013: the physical science basis. Contribution of working group I to the fifth assessment report of the intergovernmental panel on climate change. Cambridge University Press, Cambridge, S 741-866

Fuhrich M, Dosch F, Pahl-Weber E, Zillmann K (2004) Kompass für den Weg zur Stadt der Zukunft. Indikatorengestützte Erfolgskontrolle nachhaltiger Stadtentwicklung. Eine Orientierungshilfe für die kommunale Praxis. Ein ExWoSt-Forschungsfeld. BBR, Bonn

Gerstengarbe FW, Werner P (2013) Szenarios: Der Blick in eine mögliche Zukunft 2.1 Das Klimaszenario: Modellauswahl und Datengrundlage. In: Gerstengarbe FW, Welzer H (Hrsg) Zwei Grad mehr in Deutschland. Fischer Taschenbuch, Frankfurt a. M., S 31-43

Gerstengarbe FW, Werner P, Österle H, Burghoff O (2013) Winter storm- and summer thunderstorm-related loss events with regard to climate change in Germany. Theor Appl Climatol 114(3-4):715-724

Gobiet A, Suklitsch M, Heinrich G (2015) The effect of empirical-statistical correction of intensitydependent model errors on the temperature climate change signal. Hydrol Earth Syst Sci 19(10):4055-4066

Gömann H, Kreins P (2012) Landnutzungsänderungen in Deutschlands Landwirtschaft: rückläufige Anbaudiversität hat viele Ursachen. Mais 39:118-122

Gömann H, Julius C, Kreins P (2002) Quantifying impacts of different agrienvironmental policies on the environment using the regional agri-environmental information system RAUMIS. In: Environmental communication in the information society - proceedings of the 16th conference, Wien (Austria), S 210-216

Gömann H, Kreins P, Julius C, Wechsung F (2003) Landwirtschaft unter dem Einfluss des globalen Wandels sowie sich ändernde gesellschaftliche Anforderungen: interdisziplinäre Untersuchung künftiger Landnutzungsänderungen und resultierender Umwelt- und sozioökonomischer Aspekte. Schr Gesellsch Wirtsch Sozialwiss Landbaues 39:201-208

Gömann H, Kreins P, Moeller C (2004) Impact of nitrogen reduction measures on nitrogen surplus, income and production of German agriculture. Water Sci Technol 49:81-90

Gömann H, Kreins P, Breuer T (2007) Deutschland - Energie-Corn-Belt Europas? Agrarwirtschaft 56(5-6):263-271

Gömann H, Kreins P, Osterburg B, Breuer T (2007) Nutzungskonkurrenzen durch die Förderung von Biogas und anderen Energieträgern. Agrarspectrum SchrR 40:135-150

Gömann H, Kreins P, Münch J, Delzeit R (2010) Auswirkungen der Novellierung des Erneuerbare-Energien-Gesetzes auf die Landwirtschaft in Deutschland: Vortrag anlässlich der 50. In: 50. Jahrestagung Der GEWISOLA „Möglichkeiten Und Grenzen Der Wissenschaftlichen Politikanalyse“, Braunschweig, 29.09.-01.10.2010, S 189-201 
Gömann H, Kreins P, Heidecke C (2011) How global conditions impact regional agricultural production and nitrogen surpluses in the German Elbe River Basin. Reg Environ Change 11(3):663-678

Haenel H-D, Rösemann C, Dämmgen U, Freibauer A, Döring U, Wulf S, Eurich-Menden B, Döhler H, Schreiner C, Osterburg B (2016) Calculations of gaseous and particulate emissions from German agriculture 1990-2014: report on methods and data (RMD) submission 2016, Thünen Rep 39. Johann Heinrich von Thünen-Institut, Braunschweig

Hamin EM, Gurren N (2009) Urban form and climate change: balancing adaptation and mitigation in the U.S. and Australia. Habitat International 33:238-245

Hansen R, Heidebach M, Kuchler F, Pauleit S (2012) Brachflächen im Spannungsfeld zwischen Naturschutz und (baulicher) Wiedernutzung. BfN-Skripten 324, Bonn, S 151

Haylock MR, Hofstra N, Klein Tank AMG, Klok EJ, Jones PD, New M (2008) A European daily high-resolution gridded data set of surface temperature and precipitation for 1950-2006. J Geophys Res: Atmos 113(D20):D20119

Heiland S, Reinke M, Siedentop S, Träger T, Knigge M, Meyer-Ohlendorf N, Blobel D (2006) Beitrag naturschutzpolitischer Instrumente zur Steuerung der Flächeninanspruchnahme. BfN Skripten 176, Bonn, S 154

Heinzlmaier F (2013) $\mathrm{CO}_{2}$-Fußabdruck der Mineraldünger. BLW23, S 42. https://www.energieeffizienz-landwirtschaft.de/pb/site/pbs-bw/get/documents/MLR.Energieberatung/Unterlagen/03_Wissensbasis/Archiv\%20Fachartikel/Klimagase/CO2\%20Fussabdruck\%20der\%20 Minerald\%C3\%BCnger_BLW_23_2013.pdf. Zugegriffen: 19. März 2019

Hempel S, Frieler K, Warszawski L, Schewe J, Piontek F (2013) A trend-preserving bias correction - the ISI-MIP approach. Earth Syst Dynam 4(2):219-236

Henrichsmeyer W, Cypris C, Löhe W, Meudt M, Sander R, von Sothen F, Isermeyer F, Schefski A, Schleef K, Neander E, Fasterding F, Helmke B, Neumann M, Nieberg H, Manegold D, Meier T (1996) Entwicklung des gesamtdeutschen Agrarsektormodells RAUMIS96. Endbericht zum Kooperationsprojekt (Vervielfältigtes Manuskript), Forschungsbericht für das BML (94 HS 021), Bonn/Braunschweig (Germany)

Henseler M, Dechow R (2014) Simulation of regional nitrous oxide emissions from German agricultural mineral soils: a linkage between an agro-economic model and an empirical emission model. Agric Syst 124:70-82

Henseler M, Röder N, Liebersbach H, Kreins P, Osterburg B (2015) Mitigation potential and cost efficiency of abatement based subsidies for production of short rotation coppices in Germany. Biomass Bioenerg 81:592-601

Hilferink M, Rietveld P (1999) Land use scanner: an integrated GIS based model for long term projections of land use in urban and rural areas. J Geogr Inf Syst 1(12):155-177

Hirt U, Venohr M, Kreins P, Behrendt H (2008) Modelling nutrient emissions and the impact of nutrient reduction in the Weser river basin, Germany. Water Sci Technol 58:2251-2258

Hoymann J (2013) Neuere Flächennutzungsdaten - Übersicht, Vergleich und Nutzungsmöglichkeiten. BBSR-Analysen KOMPAKT 2/2013, Bonn, S 16

Hoymann J, Goetzke R (2014) Die Zukunft der Landnutzung in Deutschland - Darstellung eines methodischen Frameworks. RaumforschRaumordn 72(3):211-225

Institut für angewandte Sozialwissenschaften $\mathrm{GmbH}$ (infas), DLR Institut für Verkehrsforschung (2010) Mobilität in Deutschland 2008. Ergebnisbericht Struktur - Aufkommen - Emissionen Trends, Bonn, S 208

Intergovernmental Panel on Climate Change (IPCC) (2013) Annex II: climate system scenario tables In: Stocker TF, Qin D, Plattner GK, Tignor M, Allen SK, Boschung J, Nauels A, Xia Y, Bex V, Midgley PM (Hrsg) Climate change 2013: the physical science basis. Contribution of 
working group I to the fifth assessment report of the intergovernmental panel on climate change. Cambridge University Press, Cambridge, S 1395-1446

Isermeyer F, Brockmeier M, Gömann H, Hargens R, Klepper R, Kreins P, Offermann F, Osterburg B, Pelikan J, Salamon P, Thiele H (2006) Analyse politischer Handlungsoptionen für den Milchmarkt (Landbauforsch Völkenrode SH 300). FAL, Braunschweig

Jacobs C, Bouwman AF, Koomen E, van der Burg A (2011) Lessons learned from using landuse simulation in regional planning. In: Koomen E, Borsboom-van Beurden J (Hrsg) Landuse modelling in planning practice. Springer Netherlands, Dordrecht, S 131-149

Jacob D, Petersen J, Eggert B, Alias A, Christensen O, Bouwer L, Braun A, Colette A, Déqué M, Georgievski G, Georgopoulou E, Gobiet A, Menut L, Nikulin G, Haensler A, Hempelmann N, Jones C, Keuler K, Kovats S, Kröner N, Kotlarski S, Kriegsmann A, Martin E, Meijgaard E, Moseley C, Pfeifer S, Preuschmann S, Radermacher C, Radtke K, Rechid D, Rounsevell M, Samuelsson P, Somot S, Soussana J-F, Teichmann C, Valentini R, Vautard R, Weber B, Yiou P (2013) EURO-CORDEX: new high-resolution climate change projections for European impact research. Reg Environ Change 14:563-578

Jenssen T (2009) Klimaschutz durch städtebauliche Dichte! Möglichkeiten und Grenzen der räumlichen Steuerung von THG-Emissionen. RaumPlanung 147:281-284

Julius C, Moeller C, Osterburg B, Sieber S (2003) Indikatoren einer nachhaltigen Landwirtschaft im „Regionalisierten Agrar- und Umweltinformationssystem für die Bundesrepublik Deutschland“ (RAUMIS). Agrarwirtschaft 52:1-10

Koomen E, Rietveld P, de Nijs T (2008) Modelling land-use change for spatial planning support. Ann Reg Sci 42(1):1-10

Koomen E, Hilferink M, Borsboom-van Beurden J (2011) Introducing land use scanner. In: Koomen E (Hrsg) Land-use modelling in planning practice. The GeoJournal Library 101. Springer, Dordrecht, S 3-21

Kreins P, Gömann H (2008) Modellgestützte Abschätzung der regionalen landwirtschaftlichen Landnutzung und Produktion in Deutschland vor dem Hintergrund der „Gesundheitsüberprüfung" der GAP. Agrarwirtschaft 57:195-206

Kreins P, Gömann H, Henrichsmeyer W (2002) Auswirkungen der Vorschläge der EU-Kommission im Rahmen der Agenda 2000 Halbzeitbewertung auf Produktion, Faktoreinsatz und Einkommen der deutschen Landwirtschaft Modellanalysen auf der Grundlage des Agrarsektormodells RAUMIS, Arbeitsmaterial der Forschungsgesellschaft für Agrarpolitik und Agrarsoziologie e. V.

Kuhn U, Henneberg S, Gömann H, Kreins P, Seidel K, Tetzlaff B, Wendland F, Hirt U, Behrendt H (2008) Reduzierung diffuser Nährstoffeinträge von landwirtschaftlichen Flächen in der Flussgebietseinheit Weser. Wasser Abfall 10:20-23

Kuhr P, Haider J, Kreins P, Kunkel R, Tetzlaff B, Vereecken H, Wendland F (2013) Model based assessment of nitrate pollution of water resources on a Federal State Level for the dimensioning of agro-environmental reduction stategies: the North Rhine-Westphalia (Germany). Water Resource Manag 27(3):885-909

Kunkel R, Bogena H, Gömann H, Kreins P, Wendland F (2005) Management of regional German river catchments (REGFLUD). Water Sci Technol 51:291-299

Lasch P, Badeck FW, Suckow F, Lindner M, Mohr P (2005) Model-based analysis of management alternatives at stand and regional level in Brandenburg (Germany). For Ecol Manage 207(12):59-74

Lasch-Born P, Suckow F, Gutsch M, Reyer C, Hauf Y, Murawski A, Pilz T (2015) Forests under climate change: potential risks and opportunities. Meteorol Z 24(2):157-172

Lavalle C, Baranzelli C, Batista e Silva F, Mubareka S, Rocha Gomes C, Koomen E, Hilferink M (2011) A high resolution land use/cover modelling framework for Europe: introducing the EUClueScanner100 model. In: Murgante B, Gervasi O, Iglesias A, Taniar D, Apduhan BO (Hrsg) 
Computational science and its applications - ICCSA 2011, Part 1. Lecture notes in computer science, 6782. Springer, Berlin, S 60-75

Leppelt T, Dechow R, Gebbert S, Freibauer A, Lohila A, Augustin J, Drösler M, Fiedler S, Glat-zel S, Höper H, Järveoja J, Lærke PE, Maljanen M, Mander Ü, Mäkiranta P, Minkkinen K, Oja-nen P, Regina K, Strömgren M (2014) Nitrous oxide emission budgets and land-use-driven hotspots for organic soils in Europe. Biogeosciences 11(23):6595-6612

Lülf M (2008) Bewältigung von Klimaschutz und Klimaanpassung in Städten und städtischen Agglomerationen durch die Raumplanung? In: Klee A, Knieling J, Scholich D, Weiland U (Hrsg) Städte und Regionen im Klimawandel. E-Paper der ARL 5. Akademie für Raumforschung und Landesplanung, Hannover, S 68-85

Meehl GA, Stocker TF, Collins WD, Friedlingstein P, Gaye AT, Gregory JM, Kitoh A, Knutti R, Murphy JM, Noda A (2007) Global climate projections. In: Climate change 2007: the physical science basis. Contribution of working group I to the fourth assessment report of the intergovernmental panel on climate change. Cambridge University Press, Cambridge

Offermann F, Deblitz C, Golla B, Gömann H, Haenel H-D, Kleinhanß W, Kreins P, von Ledebur O, Osterburg B, Pelikan J, Röder N, Rösemann C, Salamon P, Sanders J, de Witte T (2014) Thünen-Baseline 2013-2023: Agrarökonomische Projektionen für Deutschland (Thünen Rep 19). Johann Heinrich von Thünen-Institut, Braunschweig, S 112

Offermann F, Banse M, Deblitz C, Gocht A, Gonzalez Mellado A, Kreins P, Marquardt S, Osterburg B, Pelikan J, Rösemann C, Salamon P, Sanders J (2016) Thünen-Baseline 2015-2025: Agrarökonomische Projektionen für Deutschland (Thünen Rep 40). Johann Heinrich von ThünenInstitut, Braunschweig

Oke T (1997) Urban climates and global environmental change. In: Thompson R, Perry A (Hrsg) Applied climatology: principles and practice. Taylor and Francis, London, S 273-287

Orlowsky B, Gerstengarbe FW, Werner PC (2008) A resampling scheme for regional climate simulations and its performance compared to a dynamical RCM. Theor Appl Climatol 92:209_ 223

Osterburg B, Kätsch S, Wolff A (2013) Szenarioanalysen zur Minderung von Treibhausgasemissionen der deutschen Landwirtschaft im Jahr 2050. No. 13. Thünen Report, 2013

Polley H, Kroiher F (2006) Struktur und regionale Verteilung des Holzvorrates und des potenziellen Rohholzaufkommens in Deutschland im Rahmen der Clusterstudie Forst- und Holzwirtschaft. Arbeitsbericht. Institut für Waldökologie und Waldinventuren, Bundesforschungsanstalt für Forst- und Holzwirtschaft, Hamburg

RAUMIS (2017) Modellergebnisse des Regionalen Agrar- und Umwelt Informationssystems RAUMIS

Rittel K, Bredow L, Wanka ER, Hokema D, Schuppe G, Wilke T, Nowak D, Heiland S (2014) Grün, natürlich, gesund: Die Potenziale multifunktionaler städtischer Räume. Ergebnisse des gleichnamigen F+E-Vorhabens (FKZ 351182 0800), BfN-Skripten 371, Bonn, S 61

Röder N, Henseler M, Liebersbach H, Kreins P, Osterburg B (2015) Evaluation of land use based greenhouse gas abatement measures in Germany. Ecol Econ 117:193-202

Roers M, Wechsung F (2015) Neubewertung der Auswirkung des Klimawandels auf den Wasserhaushalt im Elbegebiet. Hydrol Wasserbewirtsch 59(3):109-119

Rösemann C, Haenel H-D, Dämmgen U, Freibauer A, Wulf S, Eurich-Menden B, Döhler H, Schreiner C, Bauer B, Osterburg B (2015) Calculations of gaseous and particulate emissions from German agriculture 1990-2013: report on methods and data (RMD) submission 2015, Thünen Rep 27. Johann Heinrich von Thünen-Institut, Braunschweig

Scholten H, van de Velde R, Rietveld P, Hilferink M (1999) Spatial information infrastructure for scenario planning: the development of a land use planner for Holland. In: Stillwell J, Geertman S, Openshaw S (Hrsg) Geographical information and planning. Springer, Berlin, S 112-134 
Schotten C, Goetgeluk R, Hilferink M, Rietveld P, Scholten H (2001) Residential construction, land use and the environment. Simulations for the Netherlands using a GIS-based land use model. Environ Model Assess 6(2):133-143

Schuler J, Bues A, Henseler M, Krämer C, Krampe L, Kreins P, Liebersbach H, Osterburg B, Röder N, Uckert G (2014) Instrumente zur Stärkung von Synergien zwischen Natur- und Klimaschutz im Bereich Landbewirtschaftung, BfN-Skripte. Bundesamt für Naturschutz, Bonn

Schweinle J, Köthke M, Englert H, Dieter M (2018) Simulation of forest based carbon balances for Germany: a contribution to the 'carbon debt' debate. WIREs Energy Environ 7(1):1-15

Schwermer S, Preiss P, Müller W (2012) Best-Practice-Kostensätze für Luftschadstoffe, Verkehr, Strom- und Wärmeerzeugung, Anhang B der „Methodenkonvention 2.0 zur Schätzung von Umweltkosten“"

Sieber S (2003) Analyse des Risikopotenzials chemischer Pflanzenschutzmittel in der Landwirtschaft - Entwicklung und Anwendung eines modellbasierten PSM-Indikators auf das Beispiel eines bundesweiten Uferrandstreifenprogramms. Rheinischen Friedrich-Wilhelms-Universität Bonn, Bonn

Sieber S, Pannell D, Müller K, Holm-Müller K, Kreins P, Gutsche V (2010) Modelling pesticide risk: a marginal cost-benefit analysis of an environmental buffer-zone programme. Land Use Policy (Forest transitions wind power planning, landscapes and publics) 27:653-661

Spiekermann J, Franck E (Hrsg) (2014) Anpassung an den Klimawandel in der räumlichen Planung. Handlungsempfehlungen für die niedersächsische Planungspraxis auf Landes- und Regionalebene. Arbeitsberichte der ARL 11. Akademie für Raumforschung und Landesplanung (ARL), S 188

Stevens B, Giorgetta M, Esch M, Mauritsen T, Crueger T, Rast S, Salzmann M, Schmidt H, Bader J, Block K, Brokopf R, Fast I, Kinne S, Kornblueh L, Lohmann U, Pincus R, Reichler T, Roeckner E (2013) Atmospheric component of the MPI-M earth system model: ECHAM6. J Adv Model Earth Syst 5:146-172

Staupendahl K (2011) Modellierung der Überlebenswahrscheinlichkeit von Waldbeständen mithilfe der neu parametrisierten Weibull-Funktion. Forstarchiv 82:10-19

Strotmann B (1992) Analyse der Auswirkungen einer Stickstoffsteuer auf Produktion, Faktoreinsatz, Agrareinkommen und Stickstoffbilanz unter alternativen agrarpolitischen Rahmenbedingungen - Eine regionalisierte Sektoranalyse für Regionen der alten Länder der Bundesrepublik Deutschland. Wehle, Witterschick

Tetzlaff B, Kunkel R, Schäffer W, Wendland F (2007) Determination and ranking of target areas in catchments for the implementation of nitrogen reduction measures. Adv Geosci 11:69-75

Tetzlaff B, Kreins P, Kunkel R, Wendland F (2008) Area-differentiated modelling of P-fluxes in heterogeneous macroscale river basins. Water Sci Technol 55:123-131

Thünen-Institut (2013) Die zweite Bundeswaldinventur: Ergebnisdatenbank. https://bwi.info. Zugegriffen: 19. März 2019

Umweltbundesamt (UBA) (2013) Berichterstattung unter der Klimarahmenkonvention der Vereinten Nationen und dem Kyoto-Protokoll 2013. Nationaler Inventarbericht zum Deutschen Treibhausgasinventar 1990-2011. Climate change 11/2011. Umweltbundesamt, Dessau-Roßlau, S 885

Wissenschaftlicher Beirat für Agrarpolitik (WBA) (2007) Gutachten des Beirats für Agrarpolitik, November 2007, Nutzung von Biomasse zur Energiegewinnung - Empfehlungen an die Politik. https://www.bmel.de/SharedDocs/Downloads/Ministerium/Beiraete/Agrarpolitik/ GutachtenWBA.pdf?_blob=publicationFile. Zugegriffen: 19. März 2019

Wissenschaftlicher Beirat für Agrarpolitik (WBA) (2011) Stellungnahme zur geplanten Novellierung des Erneuerbare-Energien-Gesetzes. https://www.bmel.de/SharedDocs/Down- 
loads/Ministerium/Beiraete/Agrarpolitik/StellungnahmeEEG.pdf?__blob=publicationFile. Zugegriffen: 19. März 2019

Wechsung F, Wechsung M (2015) Dryer years and brighter sky - the predictable simulation outcomes for Germany's warmer climate from the weather resampling model STAR. Int J Climatol 35(12):3691-3700

Wechsung F, Wechsung M (2015b) A methodological critique on using temperature-conditioned resampling for climate projections as in the paper of Gerstengarbe FW, Werner PC, Österle $\mathrm{H}$, Burghoff O (Hrsg) (2013) winter storm- and summer thunderstorm-related loss events in Theoretical and Applied Climatology (TAC). Theor Appl Climatol 126(3-4):611-615

Weedon GP, Gomes S, Viterbo P, Shuttleworth WJ, Blyth E, Österle H, Adam JC, Bellouin N, Boucher O, Best M (2011) Creation of the WATCH forcing data and its use to assess global and regional reference crop evaporation over land during the twentieth century. J Hydrometeorol 12(5):823-848

Weingarten P (1995) Das „Regionalisierte Agrar- und Umweltinformationssystem für die Bundesrepublik Deutschland“"(RAUMIS). Berichte über Landwirtschaft 73:272-303

Weingarten P (1996) Grundwasserschutz und Landwirtschaft - Eine quantitative Analyse von Vorsorgestrategien zum Schutz des Grundwassers vor Nitrateinträgen. Schriftenreihe Landwirtschaft und Umwelt, 13, XVII, Vauk, Kiel, S 278

Weingarten P, Kreins P (2003) Maßnahmen zum Schutz des Grundwassers vor Nitrateinträgen aus der Landwirtschaft: umweltrechtliche und agrarpolitische Rahmenbedingungen. Schr Gesellsch Wirtsch Sozialwiss Landbaues 39:435-444

Wendland F, Behrendt H, Gömann H, Hirt U, Kreins P, Kuhn U, Kunkel R, Tetzlaff B (2009) Determination of nitrogen reduction levels necessary to reach groundwater quality targets in large river basins: the Weser basin case study, Germany. Nutr Cycl Agroecosyst 85:63-78

Open Access Dieses Kapitel wird unter der Creative Commons Namensnennung 4.0 International Lizenz (http://creativecommons.org/licenses/by/4.0/deed.de) veröffentlicht, welche die Nutzung, Vervielfältigung, Bearbeitung, Verbreitung und Wiedergabe in jeglichem Medium und Format erlaubt, sofern Sie den/die ursprünglichen Autor(en) und die Quelle ordnungsgemäß nennen, einen Link zur Creative Commons Lizenz beifügen und angeben, ob Änderungen vorgenommen wurden.

Die in diesem Kapitel enthaltenen Bilder und sonstiges Drittmaterial unterliegen ebenfalls der genannten Creative Commons Lizenz, sofern sich aus der Abbildungslegende nichts anderes ergibt. Sofern das betreffende Material nicht unter der genannten Creative Commons Lizenz steht und die betreffende Handlung nicht nach gesetzlichen Vorschriften erlaubt ist, ist für die oben aufgeführten Weiterverwendungen des Materials die Einwilligung des jeweiligen Rechteinhabers einzuholen.

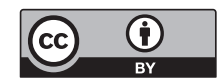

